On the Modeling, Design, and Control of Compliant Robotic Manipulators

Stefan Groothuis 

On the Modeling, Design, and Control of Compliant Robotic Manipulators

Stefan Groothuis 
Graduation committee:

Chairman:

Promotor:

Assistant promotor:

Members:

Prof. dr. ir. P. H. Veltink

Prof. dr. ir. F. J. A. M. van Houten

Prof. dr. ir. A. J. van der Schaft

Prof. dr. ir. J. L. Herder

Dr. N. G. Tsagarakis
Prof. dr. P. M. G. Apers

Prof. dr. ir. S. Stramigioli

Dr. R. Carloni

\section{University of Twente}

University of Twente

University of Groningen

Delft University of Technology

Italian Institute of Technology

DOI 10.3990/1.9789036540933

ISBN 978-90-365-4093-3

Printed by: Ipskamp Drukkers

Cover design: Stefan Groothuis

Copyright (c) 2016 Stefan Groothuis 


\title{
ON THE MODELING, DESIGN, AND CONTROL OF COMPLIANT ROBOTIC MANIPULATORS
}

\author{
PROEFSCHRIFT
}

ter verkrijging van

de graad van doctor aan de Universiteit Twente, op gezag van de rector magnificus, Prof. dr. H. Brinksma, volgens besluit van het College voor Promoties, in het openbaar te verdedigen op donderdag 2 juni 2016 om 14.45 uur

door

Stefan Sebastiaan Groothuis

geboren op 31 januari 1986

te Rhenen, Nederland 
Dit proefschrift is goedgekeurd door:

Prof. dr. ir. S. Stramigioli (promotor)

Dr. R. Carloni (assistent-promotor) 


\section{Samenvatting}

In de toekomst wordt het steeds moeilijker om persoonlijke zorg te kunnen bieden aan mensen die hulpbehoevend zijn vanwege door verscheidene aandoeningen veroorzaakte lichamelijke beperkingen, mede veroorzaakt door de toenemende vergrijzing binnen een samenleving. Het aantal zorgverleners is beperkt en de kosten van individuele en persoonlijke zorg zullen hierdoor toenemen. Hoewel deze persoonlijke zorg gewenst is, heeft het ook als nadelig gevolg dat de hulpbehoevenden afhankelijk zijn van deze zorg om de dagelijkse activiteiten te kunnen uitvoeren. Deze afhankelijkheid heeft een grote negatieve invloed op hun leefkwaliteit en zelfwaardering. Een oplossing voor deze situatie is het gebruik van robotische hulpmiddelen, reikend van taakbeperkte systemen, zoals een paginadraaier voor een boek en een eethelper, tot multifunctionele robotische armen gemonteerd op een tafel of rolstoel. Deze apparaten kunnen hulp bieden bij verscheidene taken, zoals het openen van deuren en het pakken van een drankje, maar bieden ook uitkomst bij de persoonlijke verzorging. Omdat veel huidige rolstoelgemonteerde robotarmen in sommige opzichten lijken op industriële robotarmen, die onveilig zijn omdat ze in geen enkel opzicht zijn ontworpen om menselijke zorg te bieden vanwege hun stijve constructie en positiegestuurde regeling, bestaat er behoefte aan veilige robotarmen die geschikt zijn voor deze zorg.

Dit proefschrift beschrijft allereerst verschillende aspecten van veiligheid in robotarmen en betoogt dat variabele stijfheidsactuatie, wat nog niet eerder is gebruikt in zorgverlenende robotarmen, hiervoor oplossingen biedt. Deze actuatoren bieden de mogelijkheid om niet alleen de positie van de uitgangsas te regelen, maar ook om de stijfheid aan die uitgangsas te beïnvloeden. Verschillende concepten van deze variabele stijfheidsactuatoren, gebruikmakend van verschillende principes, worden in Deel I uitgewerkt en vervolgens wordt er gekeken naar de modellering van deze robotarmen in Deel II. Modulariteit is hierbij als belangrijk doel gesteld, vanwege de grote verscheidenheid aan connecties tussen de variabele stijfheidsactuatoren en de arm. Die verscheidenheid komt tot uitdrukking in de armtopologie, waarbij in het bijzonder het articulatietype wordt beschouwd. Het articulatietype dicteert over hoeveel verschillende gewrichten het actuatorvermogen wordt verdeeld. De menselijke biceps, formeel de musculus biceps brachii, levert bijvoorbeeld tegelijkertijd vermogen aan de schouder en de elleboog, vanwege de aanhechting boven het schoudergewricht en onder het ellebooggewricht, wat wordt aangeduid met biarticulatie. Het resultaat van dit deel van het onderzoek is een model gebaseerd op grafentheorie, bestaande uit knooppunten en zijden (lijnen), waarin het herpositioneren of anders verbinden van een variabele stijfheidsactuator niets anders is dan het aanpassen van de verbindingen van één of meerdere zijden. Het 
positioneren van deze variabele stijfheidsactuatoren en het instellen van hun stijfheid, heeft invloed op de totale stijfheid die waargenomen kan worden aan het eindpunt van de robotarm. In dit proefschrift wordt gekeken naar een gestructureerde manier voor het analyseren van die stijfheid en naar het regelen van deze actuatorstijfheden om tot een zo goed mogelijke benadering te komen van een gewenste stijfheid aan het eindpunt. Een bijkomend resultaat hierbij is dat de optimale armtopologie bepaald wordt, wat geïnterpreteerd kan worden als een ontwerprichtlijn voor robotarmen met variabele stijfheid. 


\section{Summary}

In the future of the aging society it becomes increasingly difficult to provide personal care to people who are in need of help due to physical impairments caused by various diseases or disorders. The number of caregivers is limited and the cost of individual personal care will increase. Although personal care is desired, the downside is that people in need of care become dependent on caregivers for accomplishing their activities of daily living. This dependence has a big influence on one's quality of life and self esteem. A solution to this situation is the deployment of assistive robotic aids, ranging from task specific systems like a book page turner and an eating helper, to multifunctional robotic arms mounted to a table or wheelchair. These multifunctional systems can aid in various tasks like opening doors and picking up a drinking glass, as well as personal hygiene tasks. Because many of today's wheelchair mounted robotic arms are based in some aspects on existing designs for industrial applications, they are not safe to be used in human care services, mostly because of their stiff kinematic structure and the usage of position control. Therefore, there is a need for safe robotic arms that are applicable for this kind of care.

At first, this thesis describes various ways of accomplishing safety in robotic arms, and argues that using variable stiffness actuation, which has not been applied before in assistive robotic arms, is a way to accomplish that. These actuators provide the possibility to not only control the position of the actuator output, but also to influence the stiffness that can be perceived at this output. Various concepts of these actuators, using different principles, are investigated in Part I, and Part II treats the modeling of robotic arms that are driven by these actuators. Modularity was a specific goal, because of the large diversity of connections between the actuators and the arm. This diversity is expressed in the arm topology, of which the articulation type is considered in particular. The articulation type defines among which joints a connected actuator splits its output power. The human biceps, i.e., the musculus biceps brachii, applies power to both the shoulder as well as to the elbow, because of the attachment of that muscle above the shoulder joint and below the elbow joint. The result is a model based on graph theory, which consists of vertices and edges, in which the repositioning of an actuator means changing the connections of one or more edges. Positioning these variable stiffness actuators and setting their stiffnesses influences the total stiffness that can be perceived at the end effector of the robotic arm. This thesis presents a structured method of analyzing that stiffness, and treats the control of the actuator stiffnesses to optimally approximate a desired end effector stiffness. An additional result is that the optimal arm topology is determined, which can be interpreted as a design guideline for robotic arms with variable stiffness. 
1 Introduction 1

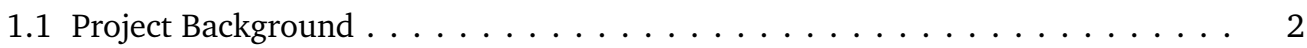

1.2 Project Goals . . . . . . . . . . . . . . . . . . . . . 3

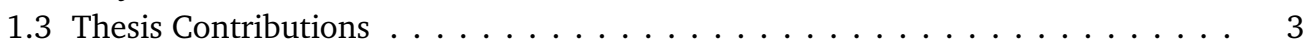

1.4 Terminology and Thesis Outline . . . . . . . . . . . . . . 3

2 Towards Novel Variable Impedance Manipulators: A Survey $\mathbf{5}$

2.1 Introduction: Assistive Robotic Arm Demands . . . . . . . . . . . . . . . 6

2.1 .1 User demands . . . . . . . . . . . . . . . . . . . 6

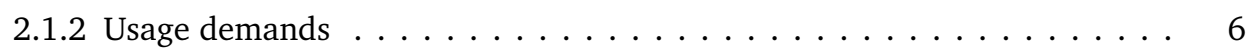

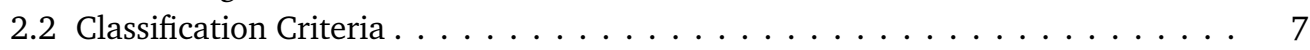

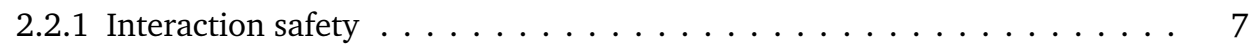

2.2 .2 Shock robustness $\ldots \ldots \ldots \ldots \ldots \ldots$

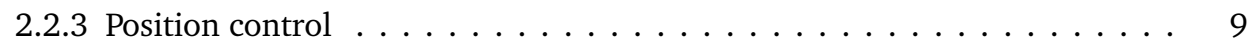

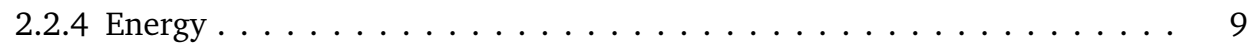

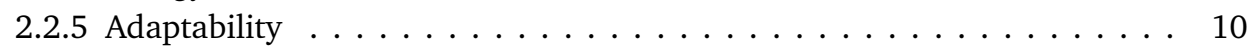

2.3 Rating Robotic Arm Suitability . . . . . . . . . . . . . . . . . . . . . . . . . . . . . . . . . . . . . .

2.3 .1 Interaction safety $\ldots \ldots \ldots \ldots \ldots \ldots \ldots$

2.3 .2 Shock robustness $\ldots \ldots \ldots \ldots \ldots \ldots \ldots$

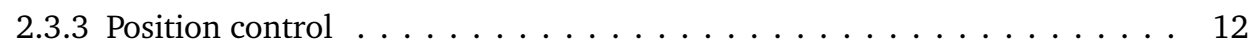

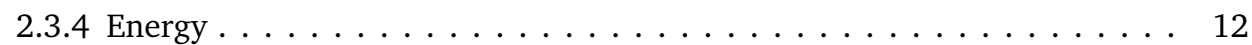

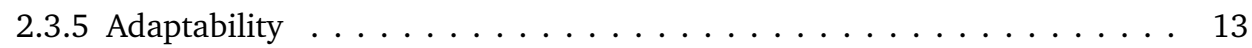

2.3.6 The drawbacks: complexity, reliability and cost . . . . . . . . . . . . . . . 13

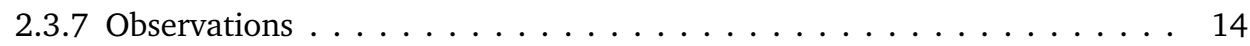

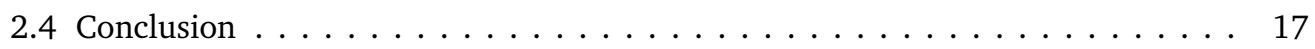




\section{Variable Stiffness Actuator Technology}

3 Lever-Arm Design: The vsaUT-II 21

3.1 The vsaUT-II Variable Stiffness Actuator . . . . . . . . . . . . . . . . . . . . 23

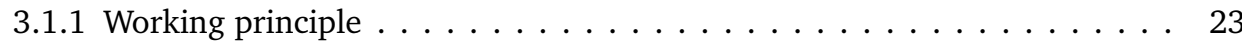

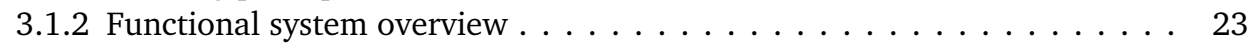

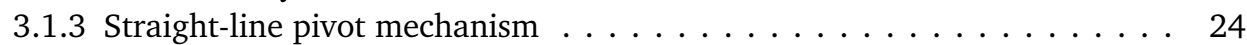

3.1 .4 Lever arm mechanism . . . . . . . . . . . . . . . . . . . 26

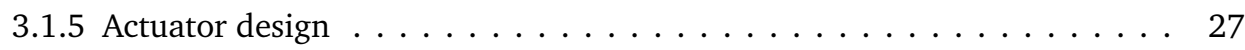

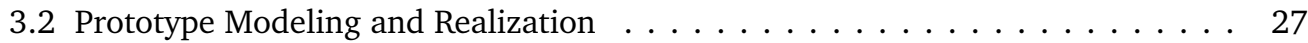

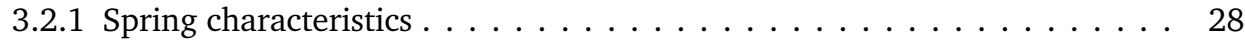

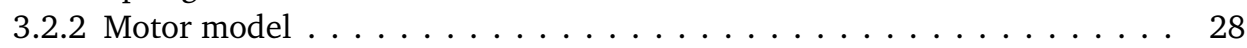

3.2 .3 Gearbox model . . . . . . . . . . . . . . . . . . . . . 29

3.2 .4 Planetary gears system . . . . . . . . . . . . . . . . . . . 30

3.2 .5 Timing belt transmission . . . . . . . . . . . . . . . . . . . 30

3.2 .6 Actuated device . . . . . . . . . . . . . . . . . . 31

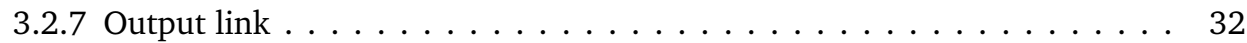

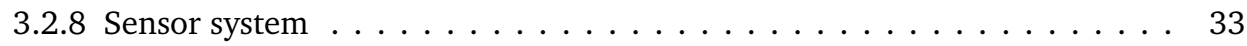

3.3 System Measurements . . . . . . . . . . . . . . . . . . . . . 34

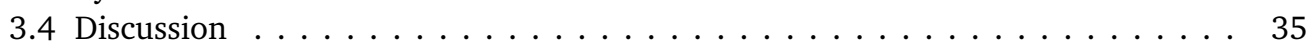

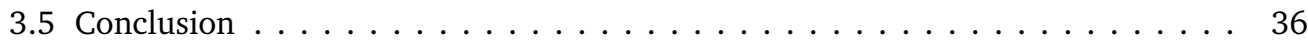

4 Leaf-Spring Design: The VSM 39

4.1 Conceptual Design . . . . . . . . . . . . . . . . . . . . . . 40

4.1.1 The variable stiffness mechanism and its working principle . . . . . . . 40

4.1 .2 Support pins . . . . . . . . . . . . . . . . . . . . 42

4.1 .3 Hypocycloid gearing mechanism . . . . . . . . . . . . . . . . . 42

4.2 Modeling . . . . . . . . . . . . . . . . . . . . . 43

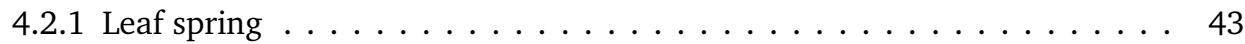

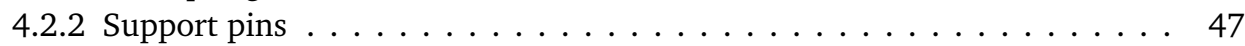

4.2 .3 Hypocycloid gearing mechanism . . . . . . . . . . . . . . . . 47

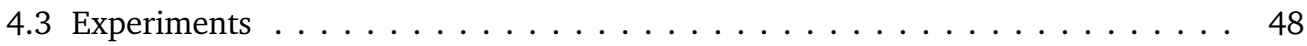

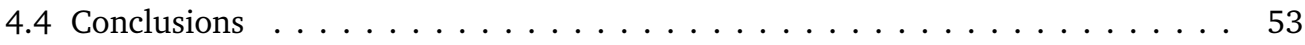

4.5 Outlook .............................. 53

5 Single-Motor Design: The SM-VSA 55

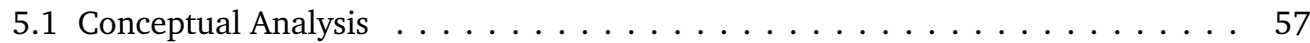

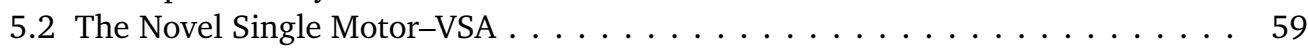

5.2 .1 Clutch analysis . . . . . . . . . . . . . . . . . . . 59

5.2 .2 Bistable switching mechanism . . . . . . . . . . . . . . . . 62

5.2 .3 The SM-VSA design and prototype . . . . . . . . . . . . . . 64

5.3 Experimental Validation $\ldots \ldots \ldots \ldots \ldots \ldots \ldots \ldots$

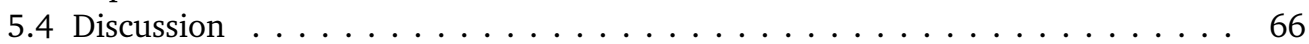

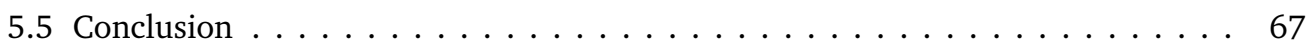

\section{Compliant Robotic Systems on Graphs}

6 Variable Stiffness Actuators on Graphs $\quad 71$

6.1 Graph Theory . . . . . . . . . . . . . . . . . 72 
6.2 Compliant Actuators on Graphs . . . . . . . . . . . . . . . . . . . . 74

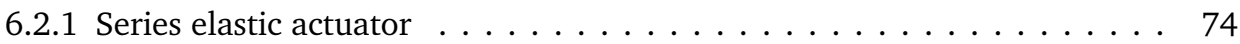

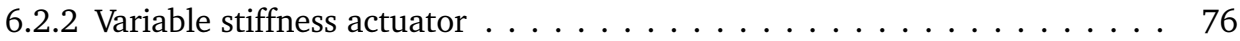

6.3 Optimal Stiffness Distribution $\ldots \ldots \ldots \ldots \ldots \ldots$

6.3 .1 System model $\ldots \ldots \ldots \ldots \ldots \ldots \ldots \ldots \ldots \ldots$

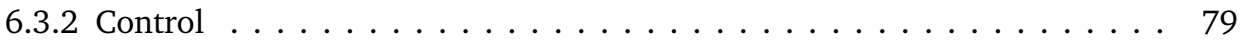

6.3 .3 Simulations and results $\ldots \ldots \ldots \ldots \ldots$

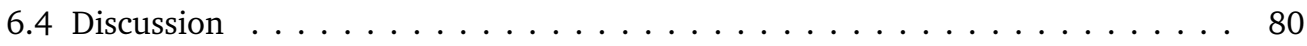

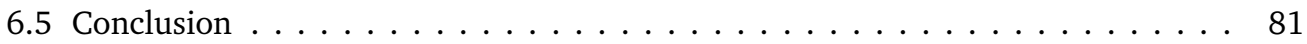

7 Compliant Robotic Manipulators on Graphs 85

7.1 Port-Hamiltonian Systems and Graph Theory . . . . . . . . . . . . . . . . . . 87

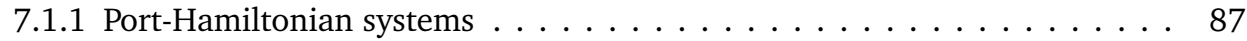

7.1 .2 Boundary and internal graph vertices . . . . . . . . . . . . 88

7.1.3 Port-Hamiltonian mechanical systems on graphs . . . . . . . . . . 88

7.2 Compliant Manipulators on Graphs . . . . . . . . . . . . . . . . . . . 89

7.2.1 Port-Hamiltonian variable stiffness actuator on graphs $\ldots \ldots \ldots$. . . . . . . . . . . . . . . . . . . . . . . .

7.2 .2 Kinematic structure . . . . . . . . . . . . . . . . . . . . . . . . . . . . . . 92

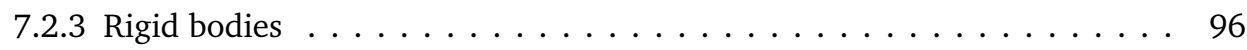

7.2 .4 Overall interconnection on graphs . . . . . . . . . . . . . . 97

7.3 Case Study: Comparison to Traditional Modeling . . . . . . . . . . . . . . . . . . . . . . . . . . . . . . . .

7.3 .1 Uniarticulated manipulator . . . . . . . . . . . . . . . . . . . . . . . . . . . . . . . . . . .

7.3 .2 Biarticulated manipulator . . . . . . . . . . . . . . . . . . 101

7.3 .3 Simulation results . . . . . . . . . . . . . . . . . . . . . . . . . . . . . . . . . . . . . . . . . . . . .

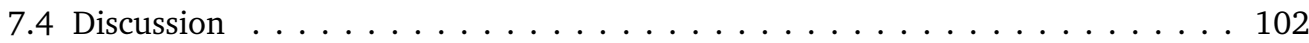

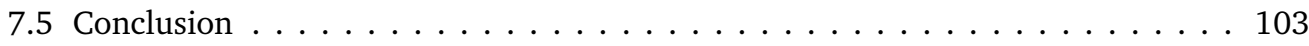

8 Manipulator Stiffness Modeling, Design, and Control 105

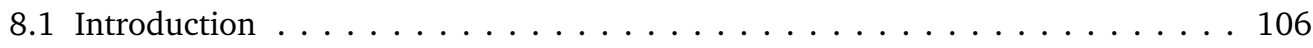

8.1 .1 Literature review . . . . . . . . . . . . . . . . 106

8.1 .2 Contribution . . . . . . . . . . . . . . . . . 107

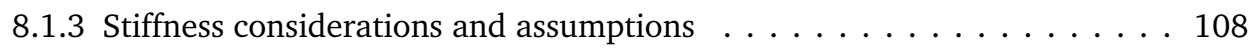

8.1 .4 Outline . . . . . . . . . . . . . . . . . . . 108

8.2 Mapping Actuation Space Stiffness to Work Space . . . . . . . . . . . . . . . . . . 108

8.2 .1 Passive springs . . . . . . . . . . . . . . . . . . . . . . . . . . . . . . . . 110

8.2 .2 Variable stiffness actuators . . . . . . . . . . . . . . . 112

8.2.3 Experimental validation: biarticulated planar mechanism . . . . . . . . 115

8.3 Controlling the Desired Work Space Stiffness . . . . . . . . . . . . . . . 118

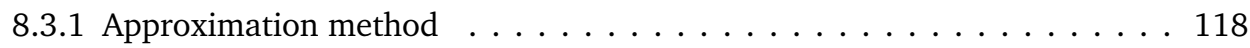

8.3.2 Stiffness Approximation Metric . . . . . . . . . . . . . . . . . . . . . 119

8.3.3 Simulations with a two degrees of freedom manipulator . . . . . . . . . . . 119

8.4 Discussion . . . . . . . . . . . . . . . . . . . . . . 121

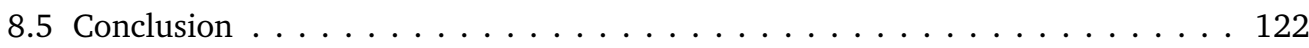

9 Conclusions $\quad 127$ 


\section{Appendices}

A Differential Geometry and Screw Theory 135

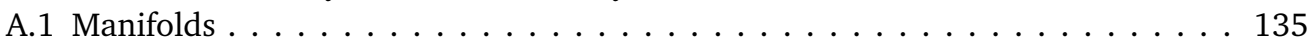

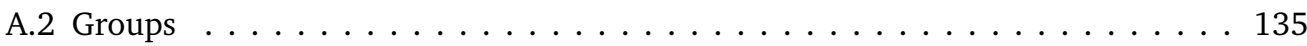

A.3 The Lie Group SE(3) . . . . . . . . . . . . . . . . . . . . . . . 136

A.4 Homogeneous Coordinates and Transformations . . . . . . . . . . . . . . 136

A.5 Elements of the Tangent Spaces . . . . . . . . . . . . . . . . . . . 137

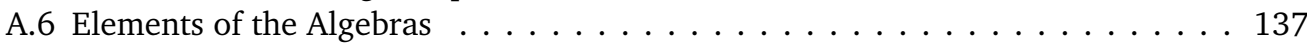

A.7 Lie Bracket . . . . . . . . . . . . . . . . . . . . . . . . . . . 139

A.8 Exponential Coordinates . . . . . . . . . . . . . . . . . . . . . . 139

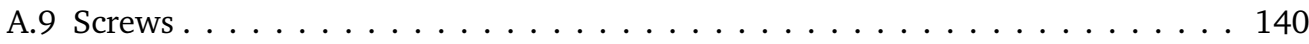

B Port-Based Modeling 141

B.1 Hamiltonian Systems . . . . . . . . . . . . . . . . . . . . . . . . . . 141

B.2 Bond Graphs . . . . . . . . . . . . . . . . . . . . . . . . . 147

C Optimal Control 149

C.1 Dynamic Programming Approach . . . . . . . . . . . . . . . . . . . . . . . . . 149

C.2 Variational Approach . . . . . . . . . . . . . . . . . . . . . . . . 150

C.3 Iterative Approach . . . . . . . . . . . . . . . . . . 150

$\begin{array}{ll}\text { D VSM Patent } & 151\end{array}$

$\begin{array}{lr}\text { Bibliography } & 179\end{array}$

$\begin{array}{ll}\text { List of Publications } & 181\end{array}$

About the Author $\quad 183$

$\begin{array}{ll}\text { Dankwoord } & 185\end{array}$ 
CHAPTER 1

Introduction

This thesis describes the work over the past four years as a result of a project on the conceptual development of a compliant robotic arm and its components. This introduction sketches the background of this project, along with the thesis goals and contributions. 


\subsection{Project Background}

Robotic systems are more and more used in our society as devices that take over dangerous, complex, or repetitive tasks from humans. Examples include the now well-known grass mowing and vacuum cleaning robots. These are relatively small systems which are not extensively interacting with people. However, when these systems do need to interact with humans, the primary concern is the safety of humans. For instance, in the alpine search and rescue project SHERPA [1], robotic devices are being developed to aid in the search and rescue of humans in harsh and highly unstructured environments, and in which the level of interaction is much higher. This level of interaction is more profound, and even fundamental, in the assistance of physically impaired people.

In the Netherlands, about 3 million people in 2011 had a physical impairment of some sort and level [2], on a total population of 16.6 million people, as shown in Figure 1.1.

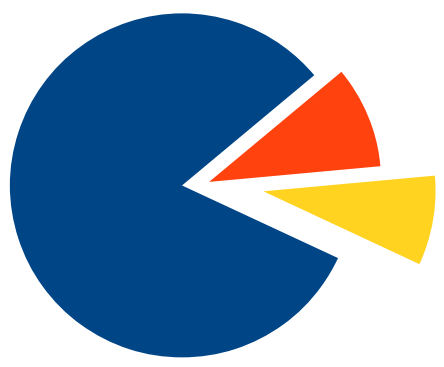

No impairment (13.6 million)

Light impairment (1.6 million)

Moderate or severe impairment (1.4 million)

Figure 1.1: The level of impairment among independently living people in The Netherlands.

These impairments may be caused by several diseases or disorders, among which are:

- Multiple sclerosis (MS), which affects the signal conduction via axons in the brain and the spinal cord;

- Muscular dystrophy (MD), which causes the death of muscle cells and tissue;

- Cerebral palsy, which damages the motor control centers;

- Rheumatic disease, which is the generic term for joint and connective tissue disorders;

- Spinal-cord lesion, where nerves have been damaged due to spinal-cord trauma.

These diseases and disorders result in limited voluntary movements and posture, which means that activities of daily living (ADL), like personal hygiene and eating, become more and more difficult, or even impossible. Very frequent, or even continuous, care is necessary given by professional caregivers, or one's spouse or relatives. This high level of dependence has a large impact on the quality of life of an impaired person.

Therefore, robotic aids, ranging from an arm support, eating helper, and an automatic book page turner, to multifunctional robotic arms mounted at a table or on a wheelchair, have been developed to assist these people. These robotic aids should enable a more independent life and increase one's quality of life considerably, together with an increase of one's self-esteem and participation in society. Although this is a very positive development, the arms that are used on wheelchairs show similarities with industrial arms that are not at all designed for operation outside a production environment. This is further elaborated in detail in Chapter 2, which is a literature review of the state of the art and a qualitative assessment of employed safety measures. It becomes clear from that study that the paradigm shift from stiff 
to soft robotic arms, which could recently be achieved by variable stiffness actuation (VSA) technology, is beneficial for assistive robotics, because certain guarantees regarding safety can be given due to the physical nature of the elasticity in variable stiffness actuators.

\subsection{Project Goals}

The goal of this project was to research the implementation of variable stiffness actuation in a robotic arm. Much focus was put on the possible arm morphologies, in particular the way in which actuators can be topologically connected. The articulation types that can be achieved were considered, which results in a tremendously large design space. Therefore, a goal was to develop a modular modeling approach of compliant manipulators based on graphs, in which the articulation types can be modeled straightforwardly. Another goal was to develop (conceptual) designs of various variable stiffness actuators. Moreover, their particular connection to a robotic arm has an influence on the stiffness capabilities of the end effector, which was to be investigated in this project.

\subsection{Thesis Contributions}

The contributions of this work can be divided in two main topics. The first is component oriented, while the second is system oriented. The contributions can be summarized as:

- the modeling, and design of various variable stiffness actuators and mechanisms, namely:

- the vsaUT-II, showing an infinite stiffness variation capability;

- the VSM, having the ability to uncouple the output for unlimited, free movement;

- the SM-VSA, showing a proof of concept of a VSA with only one relatively high power input motor capable of varying both the actuator's position as well as stiffness;

- the graph theoretic modeling of compliant robotic systems to obtain a modular tool to cope with the large design space of compliant manipulators:

- which is applied to a single variable stiffness actuator, as well as a network thereof;

- which is expanded to a six-dimensional model of generic chains of rigid bodies, to which variable stiffness actuators can be arbitrarily connected;

- which is used to analyze the stiffness of a manipulator with generic actuation topology, and to control the end effector work space stiffness to a desired stiffness using the arbitrarily connected VSAs.

\subsection{Terminology and Thesis Outline}

This thesis mentions the term variable stiffness actuation or actuators very often, which has been the common terminology for actuators with an adjustable physical elasticity. This term refers to partial variable impedance actuation, i.e., only variable stiffness and no variable inertia or damping. The term variable stiffness actuator does not give insight in the realization of the device, which is more accurately described by variable series elastic actuator (like the ones proposed in Part I), variable parallel elastic actuator, and variable antagonistic elastic 
actuator. This thesis does not use these more specific terms in general, but any ambiguous situation is resolved by explicitly stating the particular realization.

In the literature, the generally accepted way of referring to human muscles that span two joints is biarticulation. 'Bi' is a Latin numeral prefix to indicate 'two', or 'double'. Although there is a consensus on biarticulation, there is not a clear consensus for muscles that span only one joint. Very often, monoarticulation is used to indicate that situation, but 'mono' is a Greek numeral prefix, which would mean for consistency reasons (albeit from different subsystems of the Greek prefixes): di- or duoarticulation, triarticulation, and in general polyarticulation [3]. However, since biarticulation is so well accepted, in the author's opinion the Latin prefix system should be consistently chosen. This means uniarticulation, biarticulation, triarticulation, and in general multiarticulation, which have been used in this thesis.

The thesis, following the contributions, is split in two main topics, namely Part I on VSA technology, and Part II on modeling compliant manipulators using graphs. First, a literature survey that was done is presented in Chapter 2. It presents findings on current robotic arms and assesses their suitability for using it as an assistive system. Part I is split in three chapter, where Chapter 3 presents the vsaUT-II actuator, Chapter 4 presents the VSM actuator, and Chapter 5 presents a single-motor VSA, the SM-VSA. Part II is also split in three chapters, where Chapter 6 treats the modeling of variable stiffness actuators using graph theory, Chapter 7 presents the modeling of multibody compliant manipulators using the port-Hamiltonian modeling framework on graphs, and Chapter 8 presents the description of manipulator stiffness as a result of the compliant actuators, and proposes a method to design the actuator topology and control their stiffnesses. The thesis concludes with Chapter 9, after which some additional material is included in the appendices. 
CHAPTER 2

\section{Towards Novel Variable Impedance Manipulators: A Survey}

Assistive robotics is an increasingly popular research field, which leads to a large number of commercial and non-commercial systems aiming at assisting physically impaired or elderly users in the activities of daily living. In this chapter, five criteria are proposed, based on robotic arm usage scenarios and surveys, with which assistive robotic arms can be classified. Different possibilities and implementations to obtain each criterion are treated and examples of current assistive robotic arms are given. The implementations and systems are discussed and rated qualitatively, which leads to the observation that variable stiffness actuation offers great benefits for assistive robotic systems, despite an increase in the overall complexity.

This chapter has been previously published as:

[4] S. Groothuis, S. Stramigioli, and R. Carloni, "Lending a helping hand," IEEE Robotics and Automation Magazine, vol. 20, no. 1, pp. 20-29, 2013. (c) 2013 IEEE 
$\mathrm{P}$ EOPLE with upper extremity disabilities, caused by for instance spinal cord injury or multiple sclerosis, find themselves in need of continuous assistance and care. Currently, professional personal care is constantly necessary to perform daily tasks, e.g., personal hygiene, getting dressed, moving objects, eating and drinking. The complete dependence on care-givers has a tremendous impact on the quality of life. Therefore, the usage of robotic arms for the assistance of physically impaired people becomes increasingly popular. Consequently, robotic arms are used to perform everyday tasks that the user cannot perform with his/her own arms anymore. This not only improves the independence and general quality of life, but also decreases societal expense [5].

\subsection{Introduction: Assistive Robotic Arm Demands}

\subsubsection{User demands}

Several studies have been done to assess and gain insight in the possible usage scenarios of potential robotic arm users. Four pre-development and five post-development surveys on the usage of assistive robotic arms are reviewed in [6], where it is concluded that users give the highest task priority to picking up objects from the floor or shelf, and carrying objects. Moderate or high priority is given to eating/drinking, preparing food/drinks, personal hygiene, and leisure and recreation. Since leisure and recreation activities are user specific, the assistive device should be able to assist in a broad range of tasks. This creates the need for a mobile device instead of a fixed workstation, capable of functioning in a variety of unstructured environments. In [7], through a user and care-giver survey over a six month period, twelve desirable tasks for robotic arms are found, that can be classified into five groups according to the previous prioritized tasks: eating/drinking, personal hygiene (e.g., shaving, washing face), picking up/manipulating objects, personal mobility (e.g., opening doors, operating switches) and leisure/work (e.g., playing games, changing CDs).

The reviewed surveys seem to agree that, generally, users want to be able to perform activities of daily living with assistive robotic arms in highly unstructured environments, which provides them with greater independence and mobility. The majority of potential users state they would consider adopting such assistive devices $[8,9]$.

A user should be able to control a robotic arm easily by a proper interface, and considerable research effort is put into finding suitable solutions. However, this chapter does not focus on the requirements of assistive robotic arms from a user's perspective, but focuses on properties of the arm itself that are beneficial in its assistive usage instead. Although prior work has been done showing trends in the development and evaluation of assistive robotics [10], an assessment based on mechanical arm properties is not yet present in the literature.

\subsubsection{Usage demands}

A high level of human-robot interaction is required when an assistive robotic arm is used, for instance, for eating/drinking or personal hygiene tasks. Safe behavior is the most important feature during such interaction in nominal situations, but this safety should also be guaranteed in case of accidental arm collisions with the environment. Besides safety, when objects are manipulated/picked up and in case of autonomous operation, proper position control of the arm by the user is required. Moreover, since, in general, there is a need for a mobile assistive system instead of one that is fixed at a workplace, the energy consumption of such assistive systems becomes increasingly important. Proper design considerations and highefficiency electronics contribute to reducing the energy consumption, but being able to store 
externally injected energy, e.g., upon impacts, and being able to shape the intrinsic dynamics, can also considerably reduce the energy consumption. Assistive robotic arms are used in highly unstructured environments for fulfilling a broad range of tasks, and, therefore, demands on safety and performance vary. Being able to adapt to these demands provides usage flexibility and safety. Therefore, a classification and rating of assistive robotic arms can be done according to the following five criteria:

1. Interaction Safety: associated to the level of human safety when a human interacts with a robot;

2. Shock Robustness: covers the robustness of the robotic system upon high impact shocks;

3. Position Control: accounts for accurate and repeatable positioning capabilities;

4. Energy: associated to storing/reusing impact energy and dynamics shaping;

5. Adaptability: describes adaptability of systems with respect to the dynamic trade-off between performance and safety, which may be necessary under the influence of arbitrary environments and conditions.

Even if this might not be an exhaustive list of criteria for the full classification and rating of the suitability of a robotic system for assistive purposes, these criteria do capture fundamental properties that are clearly worth analyzing. These five criteria are discussed in Section 2.2. Possible implementations per criterion, as found in the literature, are elaborated and examples are given. In Section 2.3 the observed results are discussed and a qualitative rating of current systems is given. An overview of this rating, along with system properties, is summarized in a table. Concluding remarks are given in Section 2.4.

\subsection{Classification Criteria}

\subsubsection{Interaction safety}

Guaranteeing safe behavior of an assistive robotic system has a high priority during its development. Since safe behavior is not directly measurable, several safety criteria have been defined in the literature to assess certain safety properties, e.g., Gadd Severity Index (GSI), Lateral Pelvic Acceleration (LPA), Thoracic Trauma Index (TTI) and, possibly the most wellknown and widely used, the Head Injury Criterion (HIC) [11]. The HIC takes blunt impact to the head into account, measuring head acceleration and quantifying the chances of severe head injury. The head is a human's most critical part and indeed severe injury can be disastrous. However, cuts and bruises are not taken into account, but can still be very serious. Therefore, in [12], a safety norm is introduced that quantifies the intrinsic safety of an arm, which holds even when a part of the (control) system fails. More recently, a draft of the ISO 13482 standard has been released, aiming to standardize personal care safety, which also incorporates risks and hazards relating to, for instance, charging batteries, incorrect autonomous robot actions, singularity protection, speed and force restrictions, and control [13]. Since quantifying all current robotic arms in terms of these safety criteria is beyond the scope of this work, a qualitative definition of interaction safety is adopted here by stating that $a$ better interaction safety is achieved if system measures or properties limit the forces that might be applied to a human and may cause physical injury of any kind during human-robot interaction.

The interaction safety of robotic arms is a measure for its suitability of human-robot interaction and is associated to intrinsic and extrinsic, i.e., passive and active, safety measures. 
Intrinsic, i.e., passive, safety refers to measures that increase safety even when no power is supplied to the system, while extrinsic, i.e., active, safety refers to safety measures that rely on external power. Possibilities for achieving interaction safety that have been shown in the literature are limiting the arm's performance, incorporating joint backdrivability, using active impedance control and implementing intrinsically compliant joints.

Incorporating mechanical or electrical constraints in the system can lead to a higher level of safety. If, for instance, the maximum speed and force of the arm are kept low and the arm collides with a human, there is little chance of injury. Such constraints on the mechanical or electrical characteristics are defined here as performance limits. Limiting the performance of a robotic arm can be done by, for instance, limiting the arm's movement speed and acceleration in space, the end effector force, and the maximum possible payload. Speed limits in combination with low arm inertia ensures that the momentum with which a human is accidentally hit, is kept low. Examples of such systems are JACO [14] and iARM [15]. As opposed to current limits that can be changed on demand, using low power motors is a way of statically and intrinsically limiting the arm's acceleration, force and payload, as done in Weston [16]. Also Bridgit [17] and RAPUDA [18] are systems that limit their performance to increase safety. The iARM is also an example of a system that incorporates slip clutches, i.e., mechanical power transmission couplings between the actuator and joint. They disconnect, or 'slip', the transmission when the applied joint torque exceeds a certain maximum, thus limiting the end effector payload and possible force on the environment [19].

Using backdrivable joints is another way of increasing safety. Backdrivable joints do not resist to an external output motion, depending on the amount of current supplied to (and, therefore, torque supplied by) the joint motors. Therefore, a user is able to manipulate the system externally without feeling a rigid arm structure. Examples of systems using backdrivable joints are JACO and WAM Arm [20].

Active impedance control [21], often applied to intrinsically stiff joints that have a stiff coupling between the actuator and joint, is a well-known strategy of incorporating a means of safety in a robotic system. As opposed to position control where the error between a desired and actual position has to be minimized, impedance control regulates the interaction between an end effector and its environment. This way, a virtual compliance can be put at the joint level between the motor and the output, which causes the human operator to perceive a softer arm. Because of this soft behavior, the arm becomes safer to use. Examples of systems that use this type of control are KARES II, WAM Arm, Elumotion RT2 [22], DLR LWR-III [23], Modular Prosthetic Limb [24] and DLR HASy [25].

In intrinsically compliant joints the compliance, or stiffness, during interaction between the end effector and the environment is realized by a physical elastic element, e.g., a mechanical spring, that is put between the actuator and the joint, as opposed to a virtual elastic element in case of active impedance control. The stiffness felt during interaction is the physical stiffness due to the elastic element. Therefore, these joints are intrinsically safe during interaction since they do not rely on limited bandwidth controllers or possibly unreliable measurements. An example of such a system is Robonaut 2 [26], which uses series elastic joints to actuate the arm. Much like active impedance control, that mimic elastic elements of varying compliance, variable compliant joints, or variable stiffness joints [27], can adjust their physical stiffness between the actuator and the joint. In this type of joint, at least two motors are used to simultaneously control position and stiffness. Examples of such systems are DLR HASy and MIA Arm [28]. Although active impedance control can still be applied to these intrinsically compliant joints, the safe interaction behavior is guaranteed by design. 


\subsubsection{Shock robustness}

The shock robustness is a criterion for estimating the amount of damage an arm suffers upon external high impact shocks. Arm joints are the most sensitive to shocks and, therefore, should be protected. Shock robustness can be achieved by means of a system bandwidth capable of absorbing shocks, or by allowing the temporary mechanical decoupling of the actuator from the joint. Both measures can prevent damage to motors and gearboxes upon shocks.

A system bandwidth capable of reacting to arbitrary shocks can be realized only physically, i.e., by using intrinsically compliant joints. Again, these joints contain a physical elastic element between the actuator and joint, thereby converting kinetic impact energy to potential energy, as in DLR HASy, Robonaut 2 and MIA Arm.

The mechanical decoupling of the actuator from the joint upon high impacts prevents damage to the motors and gearboxes. This can be achieved by using joint slip clutches, which decouple the power transmission, thereby allowing the joint to move passively and allowing the conversion of kinetic impact energy to kinetic energy in the decoupled arm. Also backdrivable direct-drive joints, i.e., joints without non-backdrivable gearboxes, ensure that no damage occurs, since the kinetic impact energy does not have to be absorbed by the gearbox, motor or arm structure. Examples of systems with slip clutches or backdrivable joints are JACO, iARM and WAM Arm.

\subsubsection{Position control}

Besides safe interaction, maintaining accurate position control is still important for assistive robotics for object grasping, manipulation and autonomy. Position control is very effectively used and optimized in industrial applications, e.g., in the automobile industry. Typically, these robots have highly stiff structures driven by stiff joints, which means that the eigenfrequencies are high and therefore the control bandwidth can be large. For fast position control, a lower mass allowing higher accelerations is beneficial, but increases the disturbance sensitivity of the system and puts higher requirements on control. Therefore, the system mass is often a trade-off between required speed, available power and control constraints. High resolution joint encoders provide the accurate feedback and, therefore, repeatability.

Examples of systems that ensure accurate position control and repeatability by stiff structures/joints and high resolution joint encoders, are [7, 14, 15, 16, 17, 18, 20, 22, 23, 24, 29, $30,31,32,33]$.

\subsubsection{Energy}

The energy criterion relates to the capability of storing energy and reusing it at a later stage, and the possibility of using and shaping the intrinsic system dynamics to achieve a desirable motion with little actuation, which is energetically efficient. Being energy efficient is an important feature, since assistive robotics are preferably mobile and, therefore, should be able to operate independently from an external power source for a considerable amount of time.

Storing energy can only be done by incorporating a physical storage element, like a mechanical mass or spring, or any analog element in arbitrary domains, e.g., an electrical inductor or capacitor, respectively. A moving mechanical mass stores kinetic energy, so if kinetic energy at the load of a robotic arm is transferred to this mass, energy can be stored and reused later (e.g., regenerative braking). Since storing much energy in this way requires a large mass and high speed, a mechanical elastic element may be used instead. Kinetic energy is then stored as potential energy by, for instance, using mechanical springs. Examples 
of systems that have the capability to store energy using mechanical springs are DLR HASy, Robonaut 2 and MIA Arm.

Useful intrinsic system dynamics are any dynamics that require no additional external energy that can contribute to a certain desired motion or behavior, and are most often oscillatory of nature. Being able to adjust these intrinsic dynamics offers flexibility in achieving energy efficient motion, i.e., a desired motion that is primarily the consequence of intrinsic dynamics as opposed to actuation. The MIA Arm and DLR HASy are able to tune the intrinsic system dynamics by tuning their joint stiffness and, thereby, adjusting possibly useful resonance frequencies.

\subsubsection{Adaptability}

The adaptability criterion of an assistive robotic system relates to its ability to change system properties to provide a dynamic trade-off between safety and performance. This means that, for instance, safety can be decreased if more performance is needed (e.g., a higher speed has to be reached), which may be necessary due to unknown arbitrary environments and conditions. Just like interaction safety, adaptability can be achieved by either active measures, e.g., shaping performance limits and using active impedance control, or by passive measures like mechanical joint adaptations, e.g., variable stiffness joints.

Adjusting the motor current limits to reduce the maximum possible force that can be applied by the joints and, therefore, the arm's end effector, or adjusting speed and acceleration limits, increases the safety by reducing the possible force with which a human may be hit. An example of a system that uses adjustable performance (speed) limits is iARM.

Active impedance control allows the force that is applied to a load to be controlled. Since this is done actively, e.g., in software, the virtual elastic element can be tuned to a desired value required for safety, performance or other conditions. Examples of systems that have shown being able to adapt the safety and performance trade-off by means of active impedance control are KARES II, WAM Arm, Elumotion RT2, DLR LWR-III and Modular Prosthetic Limb.

Variable stiffness joints can adapt the physical stiffness perceived at the output side of a joint. Decreasing this physical stiffness means the joint is perceived as a softer spring, while increasing stiffness means that the joint is perceived as a stiffer spring. The former implies an intrinsic increase in safety and probable degradation of performance, the latter implies an intrinsic decrease in safety but improved performance. Examples of systems with variable stiffness joints are DLR HASy and MIA Arm.

\subsection{Rating Robotic Arm Suitability}

Based on the past usage surveys, general usage scenarios have been reported in the introduction in Section 2.1. Based on these preferences, five criteria to evaluate the assistive robotic arms have been proposed. Several possible implementations towards obtaining the five criteria have been treated in the previous sections and examples of current assistive robotic systems have been given that reflect these implementations. Some of the criteria are not completely independent and are related or coupled to another criterion. The energy criterion, for instance, is related to interaction safety and shock robustness if energy storing capabilities are achieved by implementing a physical elastic element: interaction safety is achieved by robust force control and shock robustness is achieved by being able to store kinetic impact energy as potential energy in the elastic element. However, a reverse does not necessarily hold: if shock robustness is provided by slip clutches, no energy storing capabilities are implicitly provided. 
The same holds for adaptability and interaction safety: suitable system adaptability, according to the criterion, means good interaction safety as a consequence, but suitable interaction safety may be achieved by static implementations (by using low power motors for instance), which results in low adaptability.

The mentioned robotic arms are organized in Table 2.1 where their function and utilization are treated, the arm mechanics and performance are given and safety and robustness properties are summarized. Since not every implementation for obtaining one of the criteria is as suitable as another, this section discusses the possibilities and rates their suitability towards the criteria in a qualitative way, shown in the bottom rows of Table 2.1 and in the plots of Figure 2.1. A system is rated ' - ' if it is unsuitable, ' $+/-$ ' if it is rated fair or adequate and ' + ' if it is rated suitable.

\subsubsection{Interaction safety}

Three implementations to increase interaction safety are found in current assistive robotic systems, i.e., limiting the arm's performance, incorporating backdrivability, and implementing compliant joints using active impedance control or incorporating intrinsic compliance.

Intrinsic safety is achieved if the measure for obtaining safety is perceived when the system is not powered, as opposed to extrinsic safety, in which that measure is actively controlled by a control system relying on possibly unreliable measurements. Therefore, the former has the greater level of safety, since it is independent of crucial measurements and control system. If no deliberate performance limits are chosen on a stiff robotic arm, it is unsafe for interaction. FRIEND [30] does not have safety measures apart from permanent magnet brakes and is, thus, rated '-'. Performance limits are intrinsically safe if low power motors are used that are under no circumstance capable of inducing too high loads. Also, slip clutches limit the maximum applicable joint torque and, therefore, end effector payload, which increases the intrinsic safety. Hence, iARM and Weston are therefore rated ' + '. Speed or current limits increase safety, but are dependent on accurate measurements. RAPUDA was targeted at safety regarding certifications and has a redundant sensor system, increasing the reliability of the measurements. Since its speed and payload performance are limited as well, it is also rated '十'.

Stiff joints can be given backdrivability, which allows the external positioning of a joint. Since it depends on the amount of current in (and, thus, applied torque by) the joint motor, which can be high, safety cannot be guaranteed. Hence, JACO and WAM Arm are rated '+/-'.

In addition to stiff joints, active impedance control might suffice with respect to safety in nominal conditions during gentle interaction without sudden disturbances. However, because of their limited bandwidth due to data processing in a digital control loop and actuator limitations, the intrinsic rigid structure of joints is felt upon a sudden disturbance and even instability may occur. Thus, active impedance cannot guarantee safety in situations where a human has to constantly interact with the robot. Therefore, KARES II, Elumotion RT2, DLR LWR-III, Modular Prosthetic Limb and WAM Arm are rated ' + /-'. A need for intrinsic safety arises, which can be achieved by passive compliance like series elastic and variable stiffness joints, i.e., DLR HASy, Robonaut 2 and MIA Arm. These systems have intrinsic interaction safety, i.e., a physical elastic element independent of external power and control bandwidth limitations, and are, thus, qualitatively rated ' + '.

\subsubsection{Shock robustness}

Shock robustness is treated as a measure for the amount of damage an arm suffers upon external high impact shocks. Slip clutches that decouple the actuators from the joint at too 
high torque prevent damage to motors and gearboxes. Also, ensuring backdrivability of joints such that the arm is free to be moved externally, prevents damage. Therefore, iARM, JACO and WAM Arm are rated '+'.

Since a control system can mimic an elastic element by active impedance control, the motors and gearboxes can be protected from these shocks. However, even when active impedance control is applied, a stiff robot is damaged at a sudden severe shock, since its bandwidth is never large enough for arbitrary shocks. Passive elastic mechanisms are robust against shocks, since their physical elastic element has an infinite disturbance bandwidth and ensures that the impact energy is not absorbed by the mechanical structure but is converted to potential energy. Therefore, Robonaut 2, MIA Arm and DLR HASy are rated ' + '. The rest of the systems in Table 2.1 are rated '-', since their joints are stiff and must absorb impact energy in their structure, most likely resulting in considerable damage.

\subsubsection{Position control}

As mentioned in Section 2.2.3, well designed stiff robotic arms with high joint encoder resolution are the best choice for accurate positioning tasks and good repeatability, as shown in industrial environments. Only high-frequency dynamics are present, which means that a large control bandwidth and, therefore, fast and accurate position control, is possible. In short, and for this reason, all systems that are actuated by stiff joints are rated ' + '. Their structural stiffness could not be assessed, but was assumed not to be a limiting factor for proper position control. In case of the absence of joint encoders, an arm has no autonomous positioning capabilities, of which the Raptor [34] is an example. Therefore, it is rated '-'.

Adding compliance to a stiff system means lowering the system's eigenfrequencies which may cause considerable oscillatory dynamics and degraded positioning performance. Variable stiffness joints can stiffen their compliance, thereby acting as stiff joints with good accuracy and repeatability when needed. Although position control of a soft arm was shown in [35], it is more challenging as opposed to stiff joint control, since the weakly damped elastic joints exhibit considerable oscillatory behavior which should be damped to obtain accurate position control. Therefore, Robonaut 2, MIA Arm and DLR HASy are rated ' + /-'.

\subsubsection{Energy}

The energy criterion treats the capability of storing energy from impacts and reusing it at a later stage and the possibilities for using and shaping the intrinsic system dynamics to yield energy efficient motions.

Stiff systems have no considerable usable intrinsic dynamics, apart from small oscillatory behavior due to finite structural stiffness and non-zero mass. Moreover, no system properties can be varied to yield varying intrinsic dynamics and since no storage element is present, no energy can be stored. These systems are not suitable for energy efficiency so all systems actuated by stiff joints are rated '-'.

Series elastic actuation has the ability to store and reuse (impact) energy by converting the kinetic energy to potential energy by compressing a mechanical spring. However, since this type of joint has one physical spring and therefore a fixed compliance, its dynamics are determined. Hence, Robonaut 2 is rated ' + /-'. Only variable stiffness joints are able to store impact energy and at the same time adjust their joint dynamics to achieve behavior that is close to a desired motion. Moreover, the energetic benefit with respect to a constant stiffness joint outweighs the additional energy consumption of an additional stiffness changing motor [36, 37, 38]. Hence, DLR HASy and MIA Arm are rated '+'. 


\subsubsection{Adaptability}

The adaptability covers the ability of a system to change properties to obtain a dynamic tradeoff between safety and performance. This trade-off may be necessary for varying usage requirements and unknown environments or conditions.

Performance limiting measures are used in various systems, e.g., limiting payload and movement speed, to ensure a certain level of safety. Also slip clutches are used to limit the maximum possible joint torque, which increases intrinsic safety. However, these limitations yield a serious static trade-off between performance on one side and human-robot interaction safety on the other side, since these are measures that may not be suitable for all situations. The adaptability of such systems, e.g., more performance at a cost of safety, is therefore insufficient $[14,15,16,17,18,29,30,31,32,33,34]$.

Active impedance controlled devices can trade off safety and performance by mimicking a stiffer or more compliant spring. However, since this is not intrinsically safe and it relies on accurate measurements and a proper control bandwidth, the KARES II, DLR LWR-III, DEKA Arm, Modular Prosthetic Limb, Elumotion RT2 and WAM Arm are rated ' + /-'. Despite a physical elastic element in the joints of Robonaut 2 , the safety and performance adaptability relies on active impedance control and is, thus, also rated ' $+/-$ '.

In case of variable stiffness joints, this trade-off can be dynamically adjusted by tuning the internal stiffness, i.e., decreasing stiffness for increased intrinsic safety and increasing stiffness for increased payload performance and accuracy. Thus, MIA Arm and DLR HASy are rated '十'.

\subsubsection{The drawbacks: complexity, reliability and cost}

While systems may be designed in a complex way such that the above criteria are met, the result might be a system that is not suitable for assistive purposes due to its level of complexity. The fundamental concern of increased complexity is the consequence on reliability. The more complex a system is designed, e.g., to achieve certain desirable properties as treated in the previous sections, the higher the probability of failure and the less reliable it becomes. Keeping systems as simple as possible very often ensures a better reliability. Another concern of complexity is maintenance cost, which might increase for complex (mechanical) solutions.

All individual systems can not be rated towards complexity and reliability without endurance testing the systems, and therefore complexity is not proposed as a criterion. However, some aspects can be discussed, of which the joint actuation implementation is the most obvious one. A stiff joint, coupling the actuator rigidly to the moving joint, has been successfully applied for decades due to its low complexity and high reliability, partly as a consequence of few components. Therefore, it is also the most inexpensive solution to build, as well as to maintain. These types of joints are not intrinsically safe and very often active impedance control is applied. The flexibility of this strategy is unsurpassed, since this control can be completely done in software and adapted very easily. However, since this method relies on accurate end effector position measurements, safety cannot be guaranteed. A constant stiffness joint, e.g., series elastic actuation, adds a physical elastic element and possible mechanical transmission to the joint actuator which introduces more mechanical components and, thereby, increases complexity and, probably, maintenance cost. Moreover, by adding the elastic element a new resonance frequency is introduced, which might make the stabilization of the system more difficult. Active impedance control can still be applied, such that one gains from the intrinsic safety of a physical elastic element (albeit dependent on the particular elastic element) and the flexibility of controlling the impedance in software. Realizing a joint with variable stiffness is more complex, since complex mechanical structures with many 
components are generally required to obtain the variable nature of the actuator output stiffness, adding even more to build and maintenance cost. Likewise, variable compliance in the robotic joint adds a variable resonance frequency and, again, might make the system stabilization more difficult. However, one does obtain unconditional intrinsic safety along with the flexibility of being able to adapt the physical joint stiffness by control.

Implementations to obtain, for instance, increased safety and shock robustness, e.g., slip clutches, might also increase complexity and wear of the system, thereby possibly decreasing its reliability. However, giving a general rating of each implementation is not possible, since the precise effects on complexity and long-term reliability are unknown.

\subsubsection{Observations}

In Figure 2.1, radar plots of the assistive robotics arms mentioned in this chapter are shown. These plots give a graphical indication of the suitability of each system towards the proposed criteria. Obviously, the larger the shaded area is, the better the system scores with respect to the five criteria. Note that the systems that could not be rated completely, are omitted. These ratings are also summarized in Table 2.1, along with system features and performance numbers.

It is observed that all systems score adequate or well at one or two of the criteria that were proposed in this chapter. It can be seen that the systems that do not score well overall are actuated by traditional stiff joints, with exceptions being iARM, JACO and WAM Arm. Their interaction safety, shock robustness and position control are adequate or good, but lack energy storing capabilities and proper adaptability of safety measures in order to increase performance in situations where safety is less important. An improvement is the series elastic actuation in Robonaut 2, which has increased safety and allows storage of energy at a small cost of position control. It is observed that MIA Arm and DLR HASy have the best rating over all five criteria, with the exception of position control, which is more challenging for weakly damped compliant systems as opposed to stiff systems. Hence, it seems that constant stiffness joints improve on stiff joints with respect to interaction safety and energetic properties, and variable stiffness joints improve on this further with respect to energetic properties and adaptability.

The assistive robotic arms of the future demand good ratings regarding all five criteria. This implies arms like the Robonaut 2, MIA Arm and DLR HASy and, therefore, ultimately the latter two, since variable stiffness joints have advantages over constant stiffness joints regarding energy and adaptability. These actuators offer the highest level of intrinsic safety of the systems that were treated, because of the soft arm behavior even when the system is not powered. Good shock robustness and the capability of storing impact energy is achieved by using a physical elastic element. Since the compliance of this element can be adjusted, intrinsic dynamics shaping is possible and adaptability of the arm's safety can be traded off with its performance, allowing versatile usage. However, these systems' drawbacks are complexity and, thus, possibly limited reliability and increased (maintenance) cost. Despite this, it is observed that, variable stiffness joints offer great benefits for assistive robotics. 


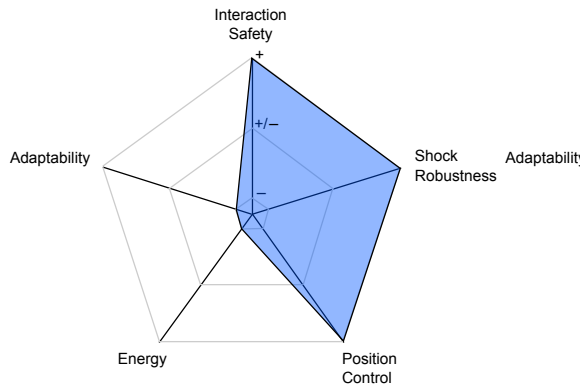

(a) iARM

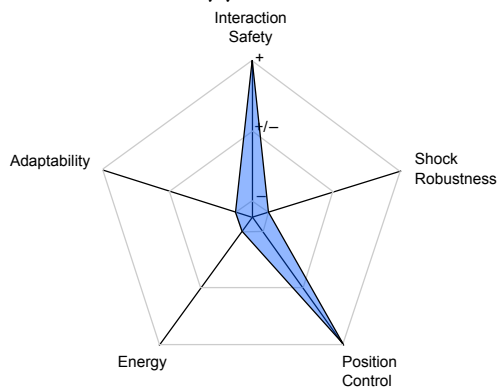

(c) Weston, RAPUDA

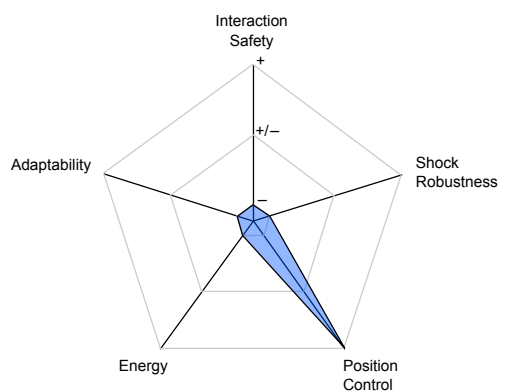

(e) FRIEND, Boston Digital Arm

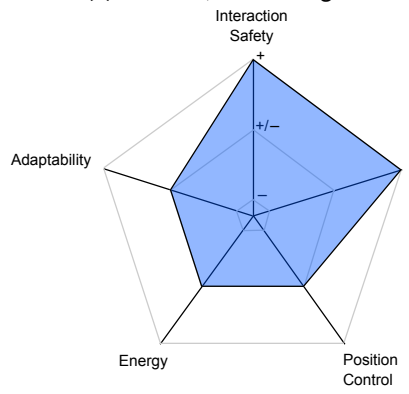

(g) Robonaut 2

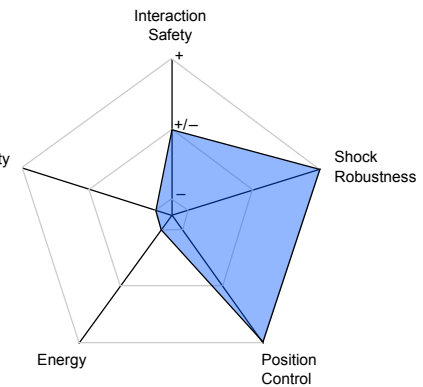

(b) JACO

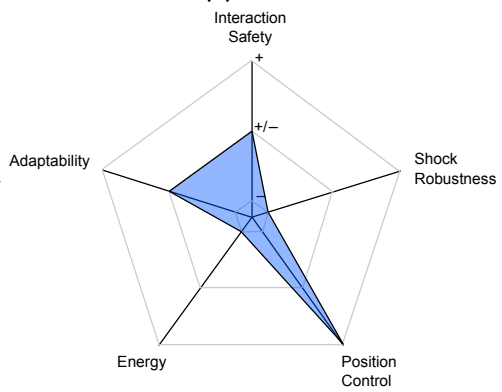

(d) KARES II, Elumotion RT2, DLR LWR-III, Modular Prosthetic Limb
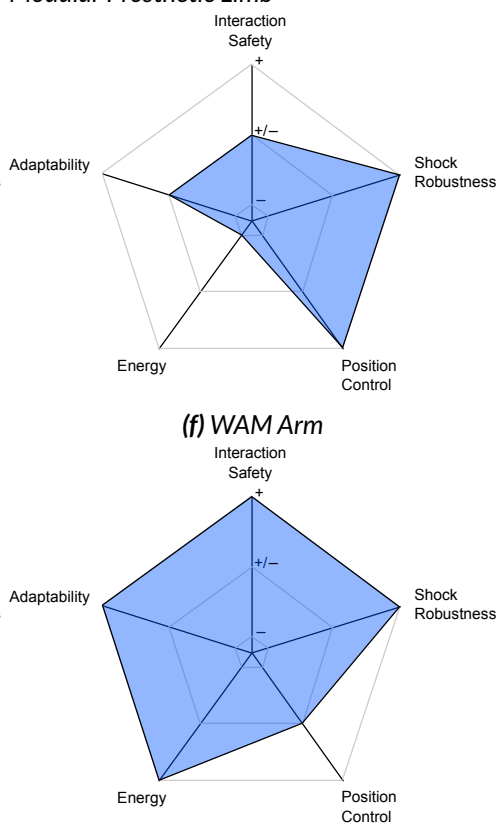

(h) MIA Arm, DLR HASy

Figure 2.1: Radar plots of assistive robotic arms mentioned in this chapter, comparing five criteria along the five axes. It can be seen that devices with variable stiffness joints have the best overall rating (the largest shaded area) for these five proposed criteria, i.e., MIA Arm and DLR HASy. 


\begin{tabular}{|c|c|c|c|c|c|c|c|c|c|}
\hline $\begin{array}{c}\text { Modular } \\
\text { Prosthetic Limb } \\
{[24]} \\
\end{array}$ & WAM Arm [20] & $\begin{array}{c}\text { Robonaut } 2 \\
{[26]}\end{array}$ & MIA Arm [28] & DLR HASy [25] & Raptor [34] & Bridgit [17] & $\begin{array}{c}\text { USF WMRA-II } \\
{[29]}\end{array}$ & Asimov [31] & DEKA Arm [33] \\
\hline Prosthesis & General purpose & $\begin{array}{c}\text { Humanlike } \\
\text { robotic } \\
\text { austronaut }\end{array}$ & $\begin{array}{l}\text { Humanlike } \\
\text { robotic arm }\end{array}$ & $\begin{array}{l}\text { Humanlike } \\
\text { robotic arm }\end{array}$ & WMRA & WMRA & WMRA & WMRA & Prosthesis \\
\hline $\begin{array}{l}\text { ADL for } \\
\text { amputees }\end{array}$ & General purpose & $\begin{array}{l}\text { Cleaning, } \\
\text { maintenance, } \\
\text { repair }\end{array}$ & $\begin{array}{c}\text { General } \\
\text { human-like arm } \\
\text { behavior }\end{array}$ & Human activities & $\mathrm{ADL}$ & $\mathrm{ADL}$ & $\mathrm{ADL}$ & $\mathrm{ADL}$ & $\begin{array}{l}\text { ADL for } \\
\text { amputees }\end{array}$ \\
\hline $\begin{array}{l}\text { Thought } \\
\text { controlled }\end{array}$ & $\mathrm{n} / \mathrm{a}$ & $\mathrm{n} / \mathrm{a}$ & $\mathrm{n} / \mathrm{a}$ & $\mathrm{n} / \mathrm{a}$ & $\begin{array}{l}\text { 10-button } \\
\text { controller or } \\
\text { 8-position } \\
\text { joystick }\end{array}$ & $\begin{array}{l}\text { Three little } \\
\text { joysticks and } \\
\text { three buttons }\end{array}$ & Computer & $\begin{array}{l}\text { Wheelchair } \\
\text { joystick }\end{array}$ & $\begin{array}{l}\text { Nerves, muscles } \\
\text { or foot pedals }\end{array}$ \\
\hline 4 & 7 & 7 & 7 & 7 & 7 & 4 & 5 & 7 & 7 \\
\hline R-P-R-P-R-PP & R-P-R-P-R-P-R & R-P-R-P-R-PP & R-P-R-P-R-PP & R-P-R-P-R-PP & P-R-P-R & R-P-P-P-R & R-P-R-P-R-P-R & $\mathrm{n} / \mathrm{a}$ & P-R-P-R \\
\hline Stiff & Stiff, direct drive & Series elastic & Variable stiffness & Variable stiffness & Stiff & Stiff & Stiff & Stiff & Stiff \\
\hline 4.5 & 27 incl. base & $\mathrm{n} / \mathrm{a}$ & 25 & $\mathrm{n} / \mathrm{a}$ & $\mathrm{n} / \mathrm{a}$ & 4.5 & 11 & 13 & 4.5 \\
\hline$\approx 1$ & 1 & 0.8 & 0.55 & $\approx 0.7$ & 1.2 & 0.65 & 1.12 & 1.5 & $\approx 1$ \\
\hline $\mathrm{n} / \mathrm{a}$ & 3 & $\geq 10$ & $\mathrm{n} / \mathrm{a}$ & $\mathrm{n} / \mathrm{a}$ & 2 & 0.5 & 3.85 & 1 & $\mathrm{n} / \mathrm{a}$ \\
\hline n/a & 3 & 2 & 1 & $\mathrm{n} / \mathrm{a}$ & $\mathrm{n} / \mathrm{a}$ & $\mathrm{n} / \mathrm{a}$ & $\mathrm{n} / \mathrm{a}$ & $\mathrm{n} / \mathrm{a}$ & $\mathrm{n} / \mathrm{a}$ \\
\hline $\mathrm{n} / \mathrm{a}$ & $\mathrm{n} / \mathrm{a}$ & $\mathrm{n} / \mathrm{a}$ & 1.88 & 10.5 & $\mathrm{n} / \mathrm{a}$ & $\mathrm{n} / \mathrm{a}$ & $\mathrm{n} / \mathrm{a}$ & 6.28 & $\mathrm{n} / \mathrm{a}$ \\
\hline- & $\begin{array}{l}\text { Back-drivable } \\
\text { joints }\end{array}$ & $\begin{array}{c}\text { Constant } \\
\text { stiffness joint }\end{array}$ & 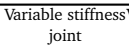 & $\begin{array}{l}\text { Variable stiffness } \\
\text { joint }\end{array}$ & $\mathrm{n} / \mathrm{a}$ & - & - & $\mathrm{n} / \mathrm{a}$ & $\mathrm{n} / \mathrm{a}$ \\
\hline $\begin{array}{l}\text { Active } \\
\text { impedance } \\
\text { control }\end{array}$ & $\begin{array}{c}\text { Robust } \\
\text { force-control } \\
\text { due to direct } \\
\text { drive, } \\
\text { independent of } \\
\text { force/torque } \\
\text { sensors }\end{array}$ & $\begin{array}{c}\text { Electromagnetic } \\
\text { failsafe brakes, } \\
\text { force/torque } \\
\text { sensors besides } \\
\text { force } \\
\text { measurement } \\
\text { via series elastic }\end{array}$ & 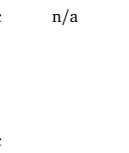 & $\begin{array}{l}\text { Active } \\
\text { impedance } \\
\text { control to } \\
\text { extend the } \\
\text { reachable } \\
\text { actuator work } \\
\text { space }\end{array}$ & $\mathrm{n} / \mathrm{a}$ & $\mathrm{n} / \mathrm{a}$ & $\mathrm{n} / \mathrm{a}$ & $\mathrm{n} / \mathrm{a}$ & $\mathrm{n} / \mathrm{a}$ \\
\hline$+/-$ & $+1-$ & + & + & + & $\mathrm{n} / \mathrm{a}$ & $\mathrm{n} / \mathrm{a}$ & $\mathrm{n} / \mathrm{a}$ & $\mathrm{n} / \mathrm{a}$ & $\mathrm{n} / \mathrm{a}$ \\
\hline - & + & + & + & + & $\mathrm{n} / \mathrm{a}$ & - & - & $\mathrm{n} / \mathrm{a}$ & - \\
\hline+ & + & $+1-$ & $+/-$ & $+/-$ & - & + & + & $\mathrm{n} / \mathrm{a}$ & + \\
\hline - & - & $+1-$ & + & + & - & - & - & - & - \\
\hline$+/-$ & $+/-$ & $+1-$ & + & + & - & - & - & - & - \\
\hline
\end{tabular}

\subsection{Conclusion}

In this chapter, the potential in using variable stiffness joints for assistive robotic devices and, in particular, arms, have been highlighted. This observation is motivated by evaluating current assistive robotic systems on five criteria, that were based on usage surveys. These surveys indicate that eating/drinking and picking up/manipulating objects are high priority tasks, which demand a high level of interaction. The properties of current assistive robotic platforms were qualitatively rated towards the proposed criteria. Most current platforms score well at two criteria, but do not score well at others. Only variable stiffness joints ensure suitable rating for all criteria and offer great benefits for assistive robotics, despite the increased overall complexity. 
PART I

\section{Variable Stiffness Actuator Technology}




\section{CHAPTER 3}

\section{Lever-Arm Design: The vsaUT-II}

In this chapter, the rotational variable stiffness actuator vsaUT-II is presented. This actuation system is characterized by the property that the apparent stiffness at the actuator output can be varied independently from its position. This behavior is realized by implementing a variable transmission ratio between the internal elastic elements and the actuator output, i.e. a lever arm with variable pivot point position. The pivot point is moved by a planetary gear mechanism, which acquires a straight motion from only rotations, thereby providing a low-friction transmission. The working principle details of the vsaUT-II are elaborated and the design is presented. The actuator dynamics are described by means of a lumped parameter model. The relevant parameters of the actuator are estimated and identified in the physical setup and measurements are used to validate both the design and the derived model.

This chapter has been previously published as:

[39] S. Groothuis, G. Rusticelli, A. Zucchelli, S. Stramigioli, and R. Carloni, "The variable stiffness actuator vsaUT-II: Mechanical design, modeling and identification," IEEE/ASME Transactions on Mechatronics, vol. 19, no. 2, pp. 589-597, 2014. (c) 2014 IEEE 
$\mathrm{C}^{\mathrm{o}}$ OMPLIANT actuators can be classified into two main categories: active and passive. In active compliant actuators, the compliance is achieved by control at a limited bandwidth and by relying on proper sensors. These sensors may fail and consequently may cause instability and safety issues during tasks in which the actuated load interacts with the surrounding environment or with humans. Passive compliant actuators, on the other hand, rely on mechanical elastic elements, placed between the internal motors and the actuated load. The internal mechanical compliance not only decouples the inertia of the motors from the load, thereby ensuring safety during any kind of human-robot interaction, but can also be used to store energy, especially during tasks in which the kinetic energy can be absorbed during impacts and released when needed, as was also shown in Chapter 2.

Variable stiffness actuators are characterized by the ability that the apparent stiffness at the actuated load can be varied independently from its position. The internal kinematic structure of a variable stiffness actuator consists, in general, of internal motors and internal elastic elements, that determine the position and the stiffness of the load and, therefore, how the internal elastic elements are perceived at the output. The output stiffness can thus be tuned to the requirements imposed by a specific task or by the environment, while the actuated load can follow a certain trajectory. Moreover, the intrinsic system dynamics can be tuned to achieve energy efficiency [40].

Considerable research effort is put into the development of variable stiffness actuators. Therefore, many different designs, based on various passive compliance principles, have been presented. Some designs rely on changing the pretension of the, in general non-linear, internal elastic elements, e.g. MACCEPA [41], VSA-II [42] and VS-Joint [43]. A characteristic of these systems is that energy is put into or extracted from the elastic elements during stiffness changes. In other designs, the number of active spring coils or the effective length of leaf springs is varied, e.g., WAM-10 [44], VSJ [45] and Jack Spring' ${ }^{\text {TM }}$ [46]. A characteristic of these systems is that an infinite stiffness configuration can be reached, but a zero-stiffness configuration cannot. The HDAU [47], AwAS [48], AwAS-II [49], CompAct-VSA [50], the vsaUT [38], the mVSA-UT [51], and [52] use a lever arm of variable effective length.

As described in $[53,54]$, when a variable transmission ratio is realized between the actuated load and the force due to the internal elastic elements, the actuator has the property that the output stiffness can be adjusted without energy injection into or extraction from the internal elastic elements. This means that all the energy supplied by the internal motors can be used to do work on the load, without being captured and, therefore, lost in the elastic elements. To realize the variable transmission ratio, a lever arm with variable effective length can be implemented. The elements acting on the lever arm are the elastic elements, the pivot point and the connection to the actuator output, on which the load applies the external force. By changing the individual positions along the lever arm, it is possible to change the transmission ratio between the elastic elements' force and the actuator output force and, therefore, to change the apparent output stiffness. Previous research showed that moving the pivot point along the lever arm minimizes the involved forces during a stiffness change [55].

In this chapter, the variable stiffness actuator vsaUT-II based on a variable transmission ratio lever arm is presented, modeled and identified. The main novelty of the actuator is the kinematic structure to move the pivot point along the lever arm, which is a modified planetary gear mechanism. This is presented in Section 3.1, together with the general working principle and the lever arm design. Section 3.2 presents the prototyping and design choices of the actuator. The lumped parameter model of the system is derived and identification of the system parameters is done. Further measurements on the realized prototype are presented in Section 3.3 to show the performance and potential of the overall system. The actuator is discussed in Section 3.4 and concluding remarks are given in Section 3.5. 


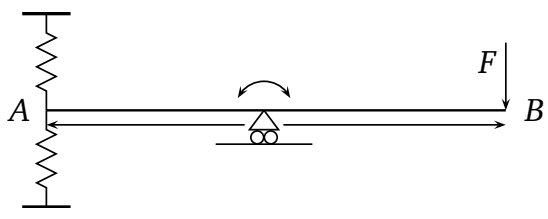

Figure 3.1: Conceptual scheme of the variable transmission ratio lever arm, obtained by means of the moving pivot point. The stiffness $K$ is zero when the pivot point is at $A$ and it is infinite when the pivot point is at $B$.

\subsection{The vsaUT-II Variable Stiffness Actuator}

In this section, the variable stiffness actuator vsaUT-II is presented, by elaborating on its working principle and showing the various functional layers separately.

\subsubsection{Working principle}

Following the discussion in [38] and [54], the design of the vsaUT-II is based on a variable transmission ratio realized between the actuated load and the force due to the internal elastic elements. By moving the pivot point position along the lever arm, it is possible to change the transmission ratio and, therefore, the apparent output stiffness felt at the load.

Figure 3.1 shows a conceptual scheme of the lever arm with variable transmission ratio, obtained by means of the moving pivot. More specifically, the figure shows a lever arm to which two elastic elements are attached at A, the output force $F$ of the load acts at B and a pivot point that can move along the entire lever arm. The output stiffness $K$, felt at the output and associated to a force $F$ and an output deflection $r$, is

$$
K:=\frac{\partial F}{\partial r}
$$

This output stiffness can be changed in a continuous way by positioning the pivot point along the lever arm. Note that: 1 ) the stiffness $K$ is zero when the pivot is located at A, since the lever arm is free to rotate about its pivot point, without influencing the elongation of the springs; 2) the stiffness $K$ is infinite when the pivot is located at $\mathrm{B}$, since there is a direct transmission of forces from the output on the pivot point.

Moving the pivot point along the lever arm to obtain a variable transmission ratio minimizes the involved forces [55] and is also used in the AwAS-II and CompAct-VSA. The HDAU and vsaUT realize a variable transmission ratio by moving the application point of the output force along the lever arm, while in the AwAS the elastic elements are moved.

\subsubsection{Functional system overview}

For a clear overview of the actuator, Figure 3.2 shows an exploded CAD view of the vsaUTII prototype in which the two fundamental actuator mechanisms are explicitly shown: the straight-line pivot mechanism and the lever arm mechanism. These layers consist of the lever arm and springs (see Figure 3.1) indicated by 7, the actuator output, 1, a planetary gear mechanism, 3 , and a motor to move the pivot, 6 . These are elaborated in detail in the following subsections. Furthermore, the rotating actuator frame, 2, rotates with respect to the fixed reference frame, 8 . The rotating frame is driven by a motor, 4 , via a timing belt, 5 . 


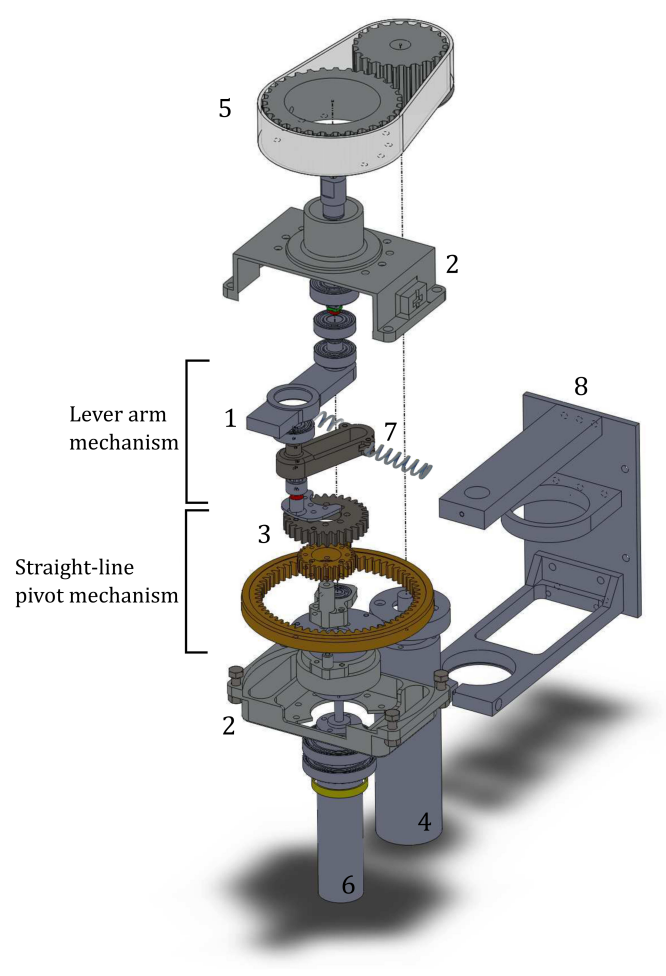

Figure 3.2: Exploded view of the vsaUT-II. The labels indicate the actuator output, 1, the rotating actuator frame, 2, the gear mechanism, 3, motor for changing equilibrium output position, 4, timing belt transmission, 5, motor for varying output stiffness, 6, the lever arm and springs, 7, and the fixed reference frame housing, 8.

\subsubsection{Straight-line pivot mechanism}

As it follows from the description of the working principle, the pivot point has to move along the lever arm and, therefore, it should follow a straight-line trajectory. The vsaUT-II prototype innovates in the way this straight-line motion is achieved, with a stiff, efficient and low-friction transmission.

The pivot is moved by a planetary gears system, as shown in Figure 3.3. It consists of a ring gear (the annulus) with pitch diameter $D_{r}$ and a planet gear with pitch diameter $D_{p}=D_{r} / 2$ to which the pivot point is connected at a distance of $D_{r} / 4$ from the center of the planet gear. Due to this precise ratio between the pitch diameters, the pivot point moves in a straight line with respect to the ring gear when the planet gear runs along the ring gear [56]. A similar (hypocycloid) mechanism is used in the HypoSEA [57], while AwAS-II uses a ball-screw and CompAct-VSA uses a rack and pinion mechanism. In a conventional planetary gear system the sun gear is in the center of the ring gear, on the same plane as the planet and ring gears. However, the necessary ratio between the pitch diameters of the gears to obtain a straight line motion prevents this and requires that the planet gear is moved by an additional connection gear, fixed under the planet gear. This way, the sun gear can be placed in the center of the ring gear in the same plane as the connection gear. As a consequence, the distance between the sun and connection gear axes is also $D_{r} / 2$. This is done by a planet carrier that carries the connection gear and the planet gear and is free to rotate about the axis which coincides with the sun gear's rotation axis. The planet carrier rotates on a motor cover, which connects the 


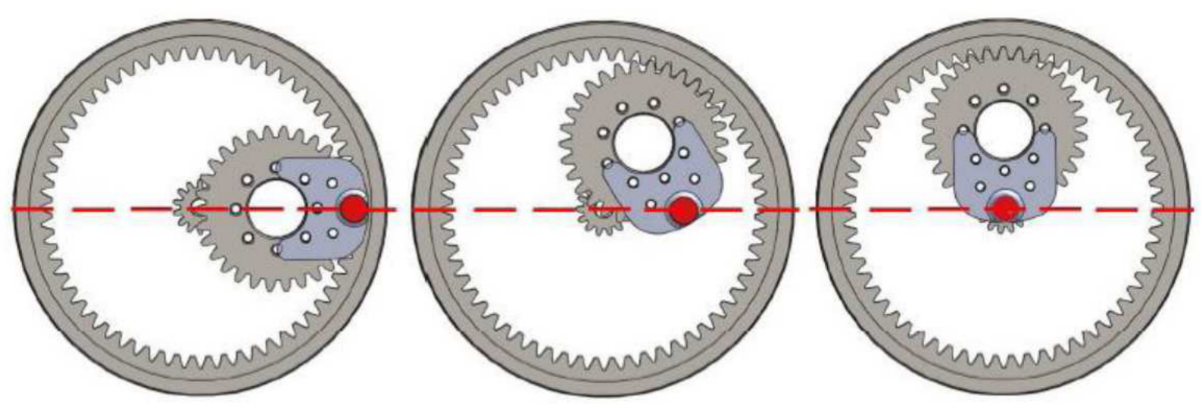

Figure 3.3: The mechanism to move the pivot along the lever arm. Since the ring gear has a pitch diameter $D_{r}$ twice as large as the pitch diameter of the planet gear $D_{r} / 2$, a point on the planet gear tracks a straight line.

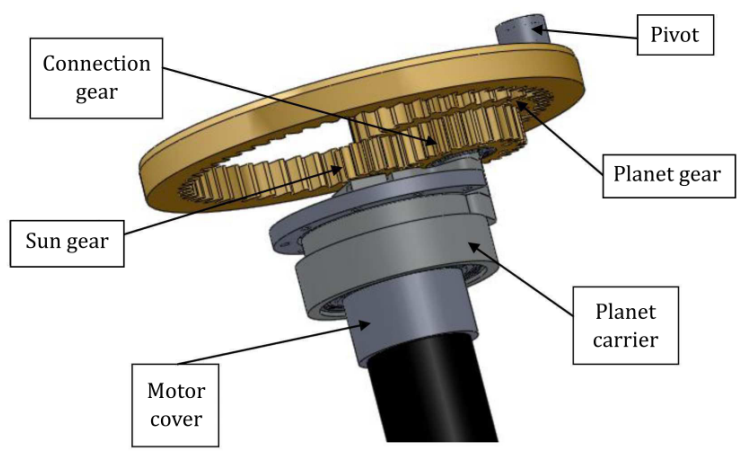

Figure 3.4: View of the planetary gears system, used to move the pivot point.

motor to the rotating frame and also functions as a housing for the ball bearing of the planet carrier. The planet carrier keeps the planet gear and connection gear at a fixed distance from the center. Figure 3.4 shows the planetary gears system used to move the pivot point. The kinematic diagram of the planetary gears system is shown in Figure 3.5. The pivot is the shaded circle indicated by coordinate $q_{1}(t), \theta_{p c}(t)$ is the angle between the center of the planet gear and straight pivot path (the dashed line), $\theta_{p}(t)$ is the angle between the line parallel to the straight pivot path and the pivot point, and $\theta_{s}(t)$ is the angle of the sun gear. The time dependency of these variables is omitted in the remainder.

The output velocity, i.e. the translational velocity of the pivot point $\dot{q}_{1}$, can be described in terms of the input velocity, i.e. the rotational velocity of the sun gear $\dot{\theta}_{s}$. In case of a conventional planetary gears system, if the ring gear is held stationary, the transmission ratio from the sun gear velocity $\dot{\theta}_{s}$ to the planet carrier velocity $\dot{\theta}_{p c}$, and thus also the transmission ratio from the respective angles $\theta_{s}$ to $\theta_{p c}$, is given by

$$
\frac{\dot{\theta}_{p c}}{\dot{\theta}_{s}}=\frac{\theta_{p c}}{\theta_{s}}=\frac{1}{1+\frac{N_{r}}{N_{s}}}
$$

where $N_{r}$ and $N_{s}$ are the number of ring gear and sun gear teeth, respectively [58]. However, since the planet gear is driven by a connection gear, the ratio changes to

$$
\frac{\dot{\theta}_{p c}}{\dot{\theta}_{s}}=\frac{\theta_{p c}}{\theta_{s}}=\frac{1}{1+\frac{N_{r}}{N_{p}} \frac{N_{c}}{N_{s}}}=\gamma
$$




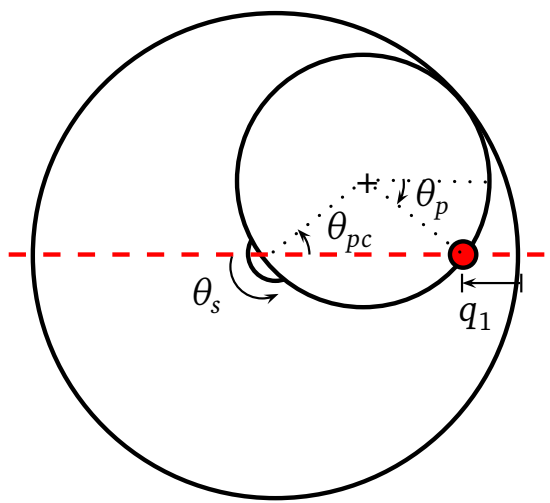

Figure 3.5: Kinematic diagram of the planetary gears system, as shown in Figure 3.3. The pivot is the shaded circle associated to coordinate $q_{1}, \theta_{s}$ is the angle of the sun gear, $\theta_{p c}$ is the angle of the planet carrier, and $\theta_{p}$ is the angle of the planet gear.

where $N_{p}$ and $N_{c}$ are the number of planet gear and connection gear teeth, respectively. Since the output velocity $\dot{q}_{1}$ is the sum of the contribution of translation velocity and rotation velocity of the planet gear, it is related to $\dot{\theta}_{p c}$ and $\dot{\theta}_{p}$ as

$$
\dot{q}_{1}=\frac{1}{2} D_{p} \sin \left(\theta_{p c}\right) \dot{\theta}_{p c}+\frac{1}{2} D_{p} \sin \left(\theta_{p}\right) \dot{\theta}_{p}
$$

Since $D_{p}=D_{r} / 2$, it holds that $\theta_{p}=\theta_{p c}$ and, therefore, $\dot{\theta}_{p}=\dot{\theta}_{p c}$. It follows that Equation (3.2) becomes

$$
\dot{q}_{1}=D_{p} \sin \left(\theta_{p c}\right) \dot{\theta}_{p c}
$$

By using (3.3) and (3.1), this part of the system is described by

$$
\dot{q}_{1}=D_{p} \sin \left(\theta_{p c}\right) \gamma \dot{\theta}_{s}
$$

The degree of freedom $q_{1}$ is then given by:

$$
q_{1}=-D_{p} \cos \left(\theta_{p c}\right)+D_{p}=-D_{p} \cos \left(\gamma \theta_{s}\right)+D_{p}
$$

Equations (3.4) and (3.5) describe the output velocity $\dot{q}_{1}$ and position $q_{1}$, respectively, as a function of the input velocity $\dot{\theta}_{s}$ and the angle $\theta_{s}$.

The usage of this mechanism ensures that a perfect translation motion is obtained from only a rotation, which means that only rotation actuators are needed. Furthermore, the mechanism is scalable, as shown by the mVSA-UT prototype [51].

\subsubsection{Lever arm mechanism}

Figure 3.6 shows a top view of the internals of the rotating actuator frame with the connection between the lever arm, the springs and the pivot. The pivot, with degree of freedom $q_{1}$, moves in a straight line of length $l$ along the lever arm. One end of the lever arm is connected to the output (the blue schematic overlay) that rotates around the center of the frame and the other end is connected to two linear extension springs, antagonistically attached and connected to the rotating actuator frame. The ring gear of the planetary gear mechanism is connected to the rotating actuator frame as well, with degree of freedom $q_{2}$. Rotating the frame with respect to the fixed reference frame $\left(\dot{q}_{2} \neq 0\right)$ corresponds to the rotation of the equilibrium output 


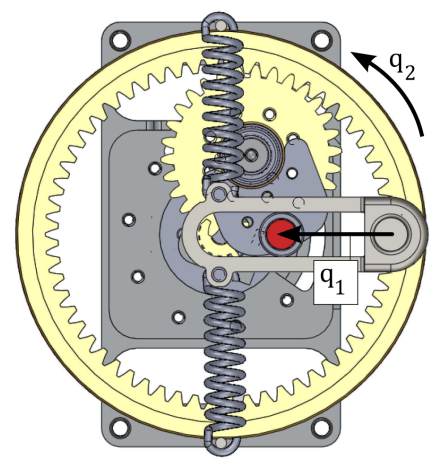

(a) Springs only pretensioned.

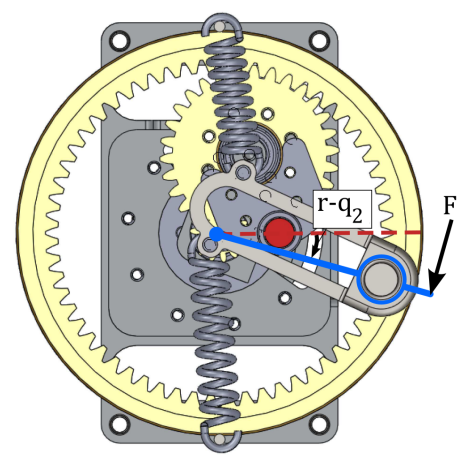

(b) Springs tensioned due to the load on the output.

Figure 3.6: View of the lever arm mechanism connected to the springs. Degree of freedom $q_{1}$ is the pivot location, $q_{2}$ is the rotation of the actuator frame (consisting of everything shown in the figure) with respect to the fixed reference frame, and $r-q_{2}$ is the deflection of the output from the equilibrium output position. It can be seen that when a load $F$ is applied to the output, the lever arms rotates around the pivot causing the spring tension to change.

position $q_{2}$ and the rotation of the straight pivot path $q_{1}$. Two different scenarios are depicted in Figure 3.6: (a) shows the pretensioned springs and (b) shows the lever arm, rotated around the pivot point due to an output load $F$ that changes the tension of the springs.

The degree of freedom $q_{1}$ determines the output stiffness setting, which, for the vsaUT-II, is given by [55]

$$
K:=\frac{\partial F}{\partial r}=2 k l^{2} \frac{\left(l-q_{1}\right)^{2}}{q_{1}^{2}} \cos \left(2\left(r-q_{2}\right)\right)
$$

where $k$ is the elastic constant of the internal elastic elements, i.e. linear mechanical extension springs, $l$ is the lever arm length along which the pivot can be moved with degree of freedom $q_{1} \in[0, l]$, the degree of freedom $q_{2}$ is the angle of the rotating actuator frame and $r$ is the output deflection, both with respect to the fixed reference frame.

Due to this particular design of the variable transmission ratio lever arm, the output stiffness can be changed without affecting the elongation of the springs. This not only holds at the actuator equilibrium position $r-q_{2}=0$, but also for a relative output position $r-q_{2} \neq 0$ [38].

\subsubsection{Actuator design}

Figure 3.7 shows a CAD drawing and the physical prototype, with labels as in Subsection 3.1.2. The desired specifications, used during the design process, are reported in Table 3.1. The active rotation angle is the maximum amplitude of the output motion $r$ and the passive rotation angle $r-q_{2}$ is the maximum angle of the output at minimum output stiffness.

\subsection{Prototype Modeling and Realization}

This section focuses on the model of the prototype and its realization. First, the choice of springs for the prototype is elaborated. Then, from Section 3.2.2, the lumped parameter 


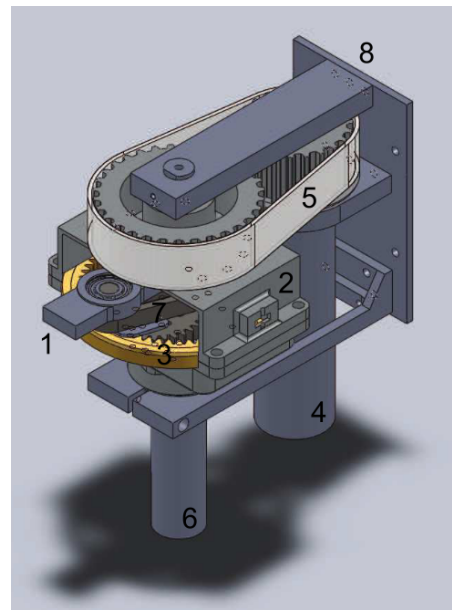

(a) CAD drawing.

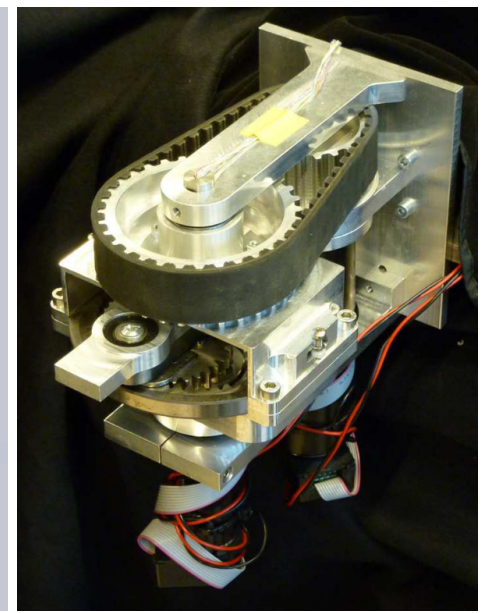

(b) Physical prototype.

Figure 3.7: CAD view and photo of the vsaUT-Il variable stiffness actuator (labels as in Figure 3.2).

model of the actuator is presented as various subsystems, and, where applicable, the measurements to find the parameters associated to the dominant behavior of the system are presented.

\subsubsection{Spring characteristics}

The output stiffness of the actuator is given by (3.6). This equation is dependent on the elastic constant $k$ of the springs that are used in the actuator. However, the total stiffness range does not depend on $k$, since the design of the actuator ensures, theoretically, an infinite stiffness range by positioning the pivot point along the lever arm. In practice, the stiffness ranges from almost zero to a very high stiffness, limited by parasitic mechanical compliance. The stiffness changing resolution (locally defined as $\frac{\partial K}{\partial q_{1}}$ ), however, does change for springs with different elastic constants. Since this prototype is intended as a conceptual system, no requirements are posed on the output stiffness profile or energy storing capacity. Arbitrary springs with an elastic constant $k=1886 \mathrm{~N} / \mathrm{m}$ are used.

\subsubsection{Motor model}

Two Maxon graphite brushed DC-motors are used for actuating the two internal degrees of freedom $q_{1}$ and $q_{2}$, as described in Section 3.1. The sun gear is driven by a Maxon $24 \mathrm{~V} 20 \mathrm{~W}$

Table 3.1: Desired specifications of the vsaUT-II.

\begin{tabular}{lcc}
\hline Property & Unit & Value \\
\hline Peak torque & {$[\mathrm{Nm}]$} & 60 \\
Nominal torque & {$[\mathrm{Nm}]$} & 15 \\
Nominal speed & {$[\mathrm{rad} / \mathrm{s}]$} & $\pi$ \\
Active rotation angle & {$[\mathrm{rad}]$} & $\pi$ \\
Passive rotation angle & {$[\mathrm{rad}]$} & \pm 0.35 \\
Min. $\leftrightarrow$ max. stiffness variation time & {$[\mathrm{s}]$} & 0.6 \\
\hline
\end{tabular}


RE-25 DC-motor, connected to a Maxon GP26B planetary gearbox with speed reduction ratio 84. The torque at the output is provided by a Maxon $24 \mathrm{~V} 150 \mathrm{~W}$ RE-40 DC-motor coupled to a Maxon GP42C planetary gearbox with speed reduction ratio 156 [59]. The motor parameters are taken from the datasheets. However, friction was not specified and since it determines the no-load speed of the motor, it was iteratively tuned to a value where the simulated no-load speed of the motor model equals the specified no-load speed, upon actuation with a constant voltage of $24 \mathrm{~V}$. Table 3.2 shows the motor parameters.

\subsubsection{Gearbox model}

Since high energy efficiency is often a goal in the application of a variable stiffness actuator, an accurate energy description in the model is desired. Therefore, a gearbox model with losses is proposed here, as opposed to a lossless gearbox where only the (power-continuous) transmission ratio is taken into account. The losses are modeled as a decrease in gearbox output torque (load torque) by

$$
\tau_{\text {load }}=n \cdot \tau_{\text {in }}-\tau_{\text {loss }}
$$

where $n>1$ is the speed reduction ratio of the gearbox.

The gearbox efficiency is a function of the load torque, where losses are primarily due to friction in the bearings and meshing of the gear teeth. At the maximum continuous torque at the output of the gearbox, the efficiency is at its maximum, while greatly decreasing for small loads. The gearbox efficiency versus torque function is assumed to be an exponential function $[59,60]$, i.e.,

$$
\eta(\tau)=\eta_{\max } \cdot\left(1-e^{-\alpha \frac{\left|\tau_{0}\right|}{\tau_{\max }}}\right)
$$

where $\eta(\tau) \in(0,1]$ is the efficiency of the gearbox, $\eta_{\max } \in(0,1]$ is the maximum efficiency, $\tau_{o}$ is the ideal (lossless) output torque, $\tau_{\max }$ is the specified maximum continuous torque at the gearbox output and $\alpha$ is a scaling parameter to adjust the steepness of the exponential function. The loss torque $\tau_{\text {loss }}$ is a fraction of the input torque and is given by

$$
\tau_{\text {loss }}=n \cdot \tau_{\text {in }} \cdot(1-\eta(\tau))
$$

Besides the losses, the rotating gearbox parts have inertia specified as the moment of inertia at the motor shaft. This has also been incorporated in the model. Table 3.3 shows the parameters used in the gearbox models.

The simulated behavior of this model can be seen in Figure 3.8. The parameters of the Maxon GP26B gearbox are used to show the behavior of the gearbox while driving an inertial load. An ideal torque source applies a linearly increasing torque at the input of the gearbox. It can be seen that for small input torques the efficiency is indeed small. The efficiency increases exponentially if the torque is increased and reaches its final value of 0.59 at an input torque of $1.3 \mathrm{Nm}$, i.e. the maximum continuous torque of the gearbox. If the torque is increased further, i.e., if the gearbox is peak loaded, the efficiency does not increase.

Table 3.2: Motor model parameters.

\begin{tabular}{lccc}
\hline Parameter & Unit & Maxon RE-20 & Maxon RE-40 \\
\hline Inductance & {$[\mathrm{H}]$} & $2.38 \cdot 10^{-4}$ & $8.23 \cdot 10^{-5}$ \\
Resistance & {$[\Omega]$} & 2.19 & 0.32 \\
Gyration constant & {$\left[\frac{\mathrm{Nm}}{\mathrm{A}}\right]$} & $2.34 \cdot 10^{-2}$ & $3.02 \cdot 10^{-2}$ \\
Motor shaft inertia & {$\left[\mathrm{kg} \mathrm{m}^{2}\right]$} & $1.07 \cdot 10^{-6}$ & $1.38 \cdot 10^{-5}$ \\
Friction & {$\left[\frac{\mathrm{Nm} \mathrm{s}}{\mathrm{rad}}\right]$} & $6.4 \cdot 10^{-6}$ & $3.0 \cdot 10^{-6}$ \\
\hline
\end{tabular}




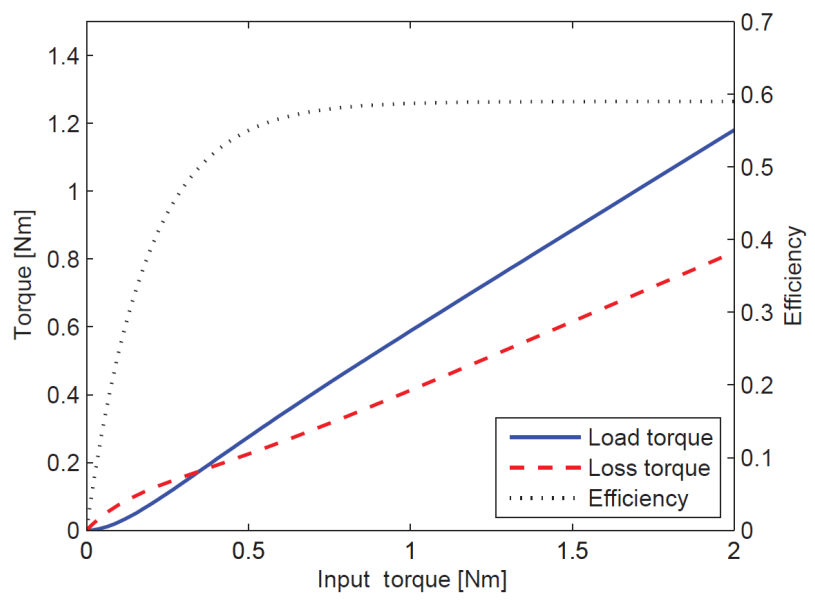

Figure 3.8: Simulation of the gearbox model where losses due to the bearings and internal gear meshing are incorporated. The efficiency is low for low input torques and increases exponentially for increasing input torques.

\subsubsection{Planetary gears system}

As described in Section 3.1.3, a linear motion is achieved by rotation through a planetary gears system. This means that the pivot can track a straight line along the lever arm, as to change the apparent output stiffness. Equations for degree of freedom $q_{1}$ and its rate of change $\dot{q}_{1}$ were derived in (3.4) and (3.5). In this specific case, the constant parameters in the equations are: $D_{p}=45.5 \mathrm{~mm}, N_{r}=60, N_{p}=30, N_{c}=31, N_{s}=14$.

The inertia of the gears at their center of rotation is approximated by the inertia of a solid disc with radius $R$ and (measured) mass $m$, i.e. $I=\frac{1}{2} m R^{2}$. For the planet gear, this means $I_{p}=3.0 \cdot 10^{-5} \mathrm{~kg} \mathrm{~m}^{2}$ at $\dot{\theta}_{p}$ and, for the sun gear, it means $I_{s}=2.4 \cdot 10^{-7} \mathrm{~kg} \mathrm{~m}^{2}$ at $\dot{\theta}_{s}$. The inertia of the displaced planet gear at $\omega_{p c}$ is approximated as $I_{p c}=9.0 \cdot 10^{-5} \mathrm{~kg} \mathrm{~m}^{2}$.

Note that this analysis describes the dynamics of degree of freedom $q_{1}$, identified by the motor, the gearbox and the planetary gear mechanism.

\subsubsection{Timing belt transmission}

As stated in Section 3.1.4, to transmit the power from the motor to the rest of the device, a timing belt transmission is used. This consists of two pulleys, i.e. an input and an output pulley, with a teeth ratio of $32 / 22 \approx 1.45$, and a Misumi P8M timing belt, pretensioned using an eccentric mechanism with $1.5 \mathrm{~mm}$ eccentricity, connecting the two pulleys. The timing belt is modeled as a linear elastic connection between the two tangential velocities of both

Table 3.3: Gearbox model parameters.

\begin{tabular}{lccc}
\hline Parameter & Unit & Maxon GP26B & Maxon GP42C \\
\hline Speed reduction ratio & - & 84 & 156 \\
Max. cont. output torque & {$[\mathrm{Nm}]$} & 1.3 & 15 \\
Max. efficiency & - & 0.59 & 0.72 \\
Scaling parameter $\alpha$ & - & 7 & 7 \\
Inertia at input & {$\left[\mathrm{kg} \mathrm{m}^{2}\right]$} & $4 \cdot 10^{-8}$ & $9.1 \cdot 10^{-7}$ \\
\hline
\end{tabular}




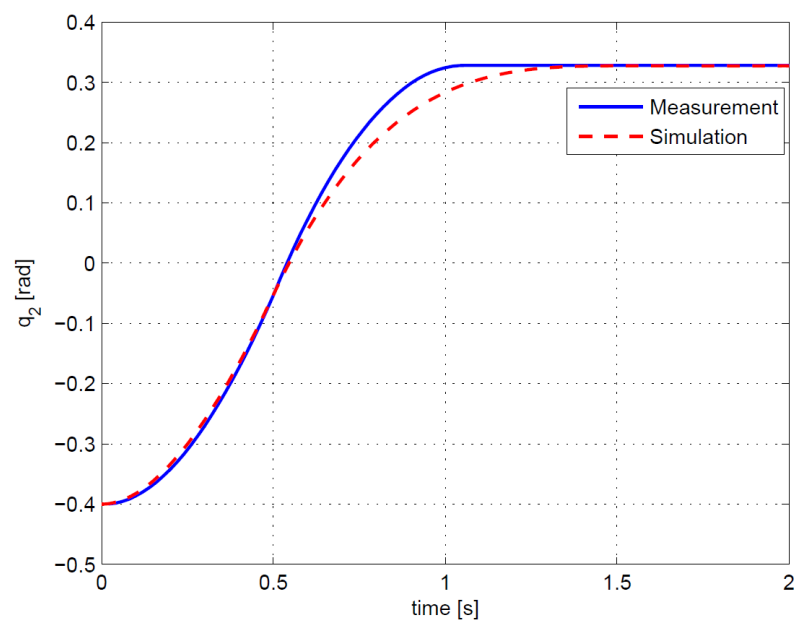

Figure 3.9: Behavior of the actuated device after actuating $q_{2}$ from an initial angle of -0.4 rad with a constant current in the motor of $0.5 \mathrm{~A}$ for $t \in[0,0.5) \mathrm{s}$. The additional friction at $q_{2}$, besides friction due to the motor and gearbox and timing belt transmission, is identified by iteratively matching simulated behavior to the measurements.

pulleys.

The actuator design enables the adjustment of the output stiffness $K$ from, theoretically, zero $\left(q_{1}=l\right)$ to infinite $\left(q_{1}=0\right)$ stiffness. The (finite) maximum stiffness of the actuator was measured and the corresponding compliance is assumed to be concentrated in the elasticity of the timing belt, and thus the internal parts are assumed rigid. The maximum output stiffness $K_{\max }$ is found to be $948 \mathrm{Nm} / \mathrm{rad}$. This can be converted to the linear elasticity in the timing belt by using the output pulley radius $r_{o p}=41 \mathrm{~mm}$, i.e., $C_{b e l t}=\frac{K_{\max }}{r_{o p}^{2}}=564 \mathrm{kN} / \mathrm{m}$.

\subsubsection{Actuated device}

The actuated device is defined as the subsystem that is moved by the degree of freedom $q_{2}$, i.e. the combination of the planetary gears system, the motor and the gearbox for the actuation of $q_{1}$, the rotating actuator frame and the output pulley. Note that the output bar should not be considered here. This part is modeled as a rotating mass subject to friction.

\section{Inertia}

The initial estimate of the inertia of the actuated device around its central axis $I_{a d}$ is based on a rotating solid disc of radius $R=45 \mathrm{~mm}$ with a measured mass of $m=1.2 \mathrm{~kg}$, i.e., $I_{a d}=1.2 \cdot 10^{-3} \mathrm{~kg} \mathrm{~m}^{2}$.

The inertia of an electric motor at its output shaft can be reflected to an equivalent inertia at its gearbox shaft by multiplying the motor inertia with the square of the gearbox speed reduction ratio. By doing this, it is shown that the device inertia $I_{a d}$ is much smaller than this transformed inertia, which means that $I_{a d}$ can be neglected, i.e., $I_{\mathrm{RE}-40} \cdot n_{\mathrm{GP} 42 \mathrm{C}}^{2}=0.34 \mathrm{~kg} \mathrm{~m}^{2} \gg$ $1.2 \cdot 10^{-3} \mathrm{~kg} \mathrm{~m}^{2}$. 


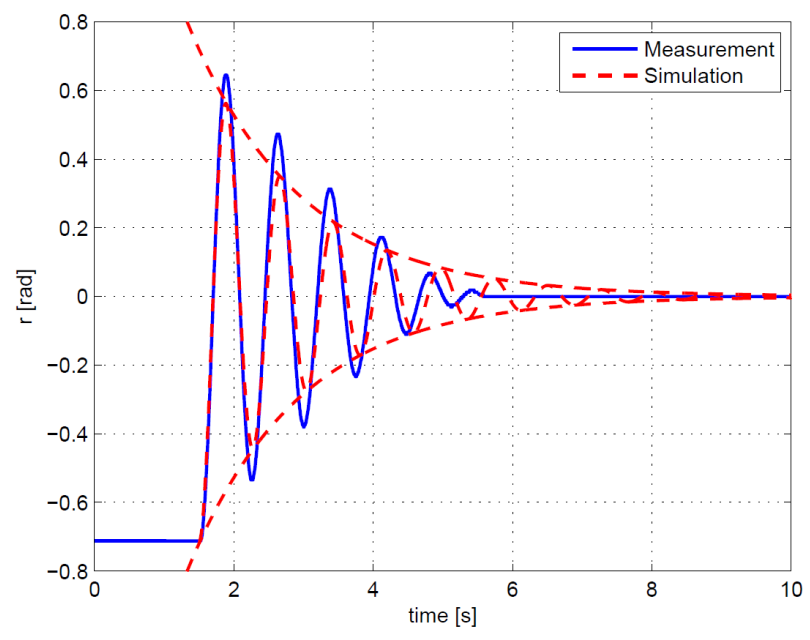

Figure 3.10: Output oscillations as measured and simulated. The measured oscillations of the output link were manually triggered, the simulated oscillations, where the exponential amplitude decrease is made explicit, are computed with identified parameters.

\section{Friction}

The friction while rotating the actuated device is modeled as linear viscous friction and assumed concentrated at degree of freedom $q_{2}$. The friction parameter was found by measuring the damping effect in the prototype after actuating $q_{2}$ from an initial angle of -0.4 rad with a constant current in the motor of $0.5 \mathrm{~A}$ for $t \in[0,0.5) \mathrm{s}$. This test has also been performed in simulations: the additional friction at $q_{2}$ in the model, besides friction due to the large motor and gearbox and timing belt transmission, has been iteratively adjusted as to match the simulated behavior to the measurement. A friction parameter of $0.55 \mathrm{Nm} \mathrm{s} / \mathrm{rad}$ has been found, as shown in Figure 3.9. Only during the decelleration phase, a deviation can be seen.

Note that this analysis describes the dynamics of degree of freedom $q_{2}$, identified by the motor and gearbox, the timing belt transmission and the actuated device.

\subsubsection{Output link}

The output link is the part where the load can be attached and during tests a solid aluminium bar was fixed to the output. Although the inertia found here is only valid for the particular setup used, the friction measurement is valid in general.

\section{Inertia}

The initial inertia estimate is based on a solid rod with length $L=350 \mathrm{~mm}$ and a measured mass $m=0.262 \mathrm{~kg}$ rotating about one of its ends, i.e., $I_{o}=1.1 \cdot 10^{-2} \mathrm{~kg} \mathrm{~m}^{2}$. The actual inertia of the output link was measured by setting the output stiffness to the most compliant setting ( $K=0.7 \mathrm{Nm} / \mathrm{rad}$, as shown in Section 3.3), pulling the output to one side, releasing it and measuring the oscillations. This behavior is shown in Figure 3.10 and it is assumed that it can be described by a harmonic oscillator (an underdamped mass-spring system), i.e.,

$$
\ddot{r}(t)+\frac{c}{I_{o}} \dot{r}(t)+\omega_{0}^{2} r(t)=0,
$$


where the undamped resonance frequency (assumed negligible different from the damped resonance frequency) is given by $\omega_{0}=\sqrt{\frac{K}{I_{o}}}$. Thus, the inertia is found by $I_{o}=K / \omega_{0}^{2}$ or $I_{o}=$ $K \Delta t^{2} / 4 \pi^{2}$, in which $\Delta t$ is the time period between two successive peaks of the oscillation. By using the first two peaks in Figure $3.10(\Delta t=0.77 \mathrm{~s})$, the inertia is found to be $I_{o}=$ $1.1 \cdot 10^{-2} \mathrm{~kg} \mathrm{~m}^{2}$, which equals the initial estimate.

\section{Friction}

Two successive peaks in Figure 3.10 also define the amount of friction in the subsystem, i.e. the damping coefficient in the model. Again, a linear friction model is assumed: the (small) linear viscous friction in the bearings causes an exponential decay $\delta$ in the amplitudes of the oscillation given by $r(t)=\alpha e^{-\delta t}$, where $\delta=\frac{1}{n T} \ln \frac{r\left(t_{0}\right)}{r\left(t_{0}+n T\right)}=0.62 / \mathrm{s}$, in which $r\left(t_{0}\right)$ is the amplitude of the peak at time $t_{0}$ and $r\left(t_{0}+n T\right)$ is the n-th peak counting from the peak at $r\left(t_{0}\right)$ (the first and fifth lower extrema were used, i.e., $\left.n=4\right)$. Since the general solution to (3.7) is an exponentially decaying harmonic oscillation and is given by

$$
r(t)=\left[A_{1} \cos (\omega t)+A_{2} \sin (\omega t)\right] e^{\frac{-c}{2 I_{0}} t}
$$

with amplitudes $A_{1}$ and $A_{2}$ and frequency $\omega$, the friction coefficient $c$ can be found

$$
\begin{aligned}
e^{-\delta t} & =e^{-\frac{c}{2 I_{o}} t} \\
c & =2 \delta I_{o}=1.4 \cdot 10^{-2} \mathrm{Nm} \mathrm{s} / \mathrm{rad}
\end{aligned}
$$

This damping coefficient is used as the coefficient of friction in the model. The simulated oscillations at the output can be seen in Figure 3.10. The measured output behavior is approximated, but decays faster than the corresponding oscillations in the model due to the linear friction model.

\subsubsection{Sensor system}

For controlling the device, sensors are needed to estimate the states of the system. By having an estimate of the degrees of freedom $q_{1}$ and $q_{2}$ and the output angle $r$, all other system states and desired properties can be estimated.

An incremental optical encoder Maxon HEDL 5540-500 with 500 counts per revolution (2000 pulses per revolution in quadrature encoding) was mounted on each of the two DCmotors to measure the relative angle of the motors. The angle of the motor $\theta$ is converted to the number of pulses $p$ by

$$
p=\left\lfloor\theta \cdot \frac{2000}{2 \pi}\right\rfloor
$$

where $\lfloor\ldots\rfloor$ indicates a floor function of the argument (the number of pulses) to the nearest lower integer number, to model the quantization process. The motor state is represented by the number of pulses from its initial position and therefore by multiplying (3.8) with $\frac{2 \pi}{2000}$, the motor angle is found again, with some lost accuracy due to the quantization. By properly taking the gearbox and the timing belt transmission into account, the degree of freedom $q_{2}$ can then be found.

An absolute magnetic encoder (Austria Microsystems AS5043) with a resolution of 10 bits was used to directly measure the output angle $r$. Similarly to the optical encoders, this sensor was modeled as a quantizer on the angle $r$ with a resolution of $\frac{360^{\circ}}{1024} \approx 0.35^{\circ} /$ step and added uncertainty due to gain mismatch errors of the AS5043 of $\pm 0.15^{\circ}$ according to the proposed model in [61]. 


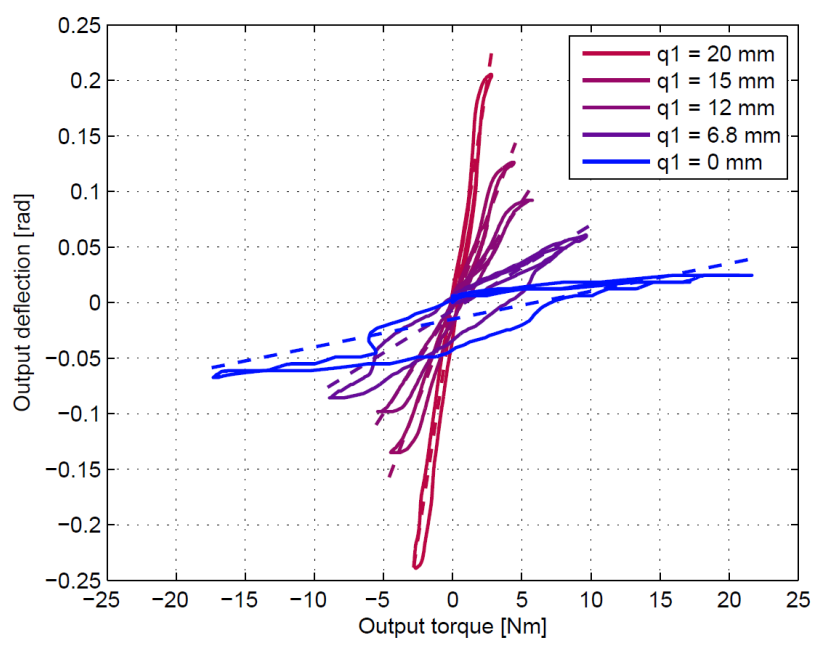

Figure 3.11: Output torque versus deflection. For various stiffness settings determined by $q_{1}$, a torque is applied on the output and the corresponding deflection is measured. The tangent to these curves at a point gives the compliance of the output at that configuration.

An absolute calibration of the system can be performed thanks to the absolute magnetic encoder. A homing action on the degree of freedom $q_{1}$ to the zero position at an unloaded system calibrates the measurement on $q_{1}$ to zero. At this setting, it holds that $q_{2}=r$. Therefore, $q_{2}$ is in its zero position and can be calibrated as such if $r$ is put in the (absolute) zero position by actuating $q_{2}$. All further incremental measurements are performed with respect to this zero position.

\subsection{System Measurements}

In this section, the vsaUT-II prototype is characterized by deriving the actuator output torquedeflection diagram. This diagram shows the output deflection $r-q_{2}$ of the actuation system as a function of the actuator output torque for various output stiffness settings, determined by $q_{1}$. The measurement is done with an ATI Mini40 F/T sensor with a maximum force measure capability of $240 \mathrm{~N}$ (SI-80-4 metric calibration) and a corresponding resolution of $1 / 25 \mathrm{~N}$. The sensor is attached to the output link at $267 \mathrm{~mm}$ from the center of rotation and an external force is applied to cause an output deflection. A measurement is started in the equilibrium position and is performed as a continuous motion in the positive and negative direction, such that the hysteresis effect caused by energy dissipation (e.g. friction) is shown. The output deflection is measured with the magnetic encoder. The applied torque is calculated by multiplying the measured force and the sensor distance. This measurement is repeated for different pivot positions, i.e. for different output stiffnesses. Note that these measurements are done at the output and, therefore, do not provide transmission efficiency figures, such as the gearbox efficiency as elaborated in Section 3.2.

Figure 3.11 shows the result of five of these measurements, namely for pivot positions $q_{1}$ at $20,15,12,6.8$ and $0 \mathrm{~mm}$. The curves show interpolated data to compensate quantization noise. A dashed line shows the linear least-squares approximation to the curves, of which the slope represents the output compliance. The reciprocal of the linearly approximated slopes represents output stiffness, which is plotted in Figure 3.12. In particular, the solid line is the 


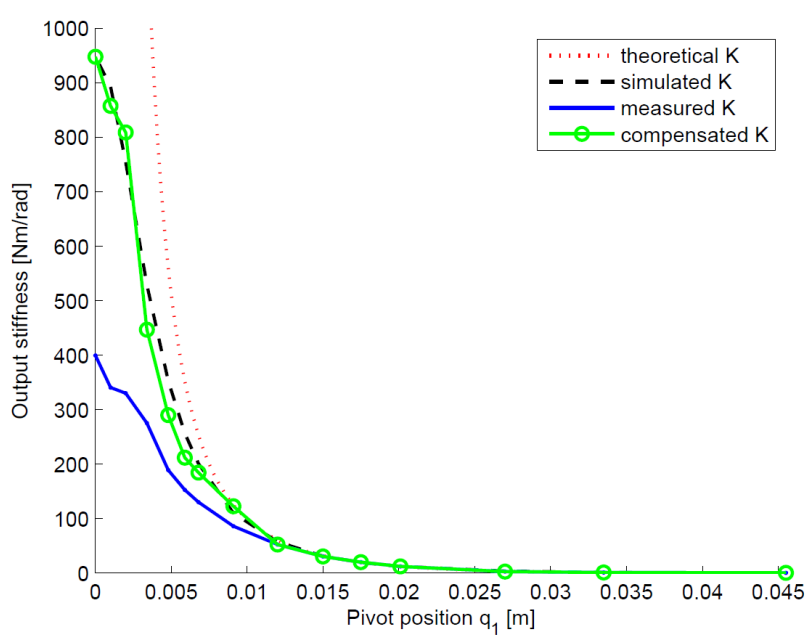

Figure 3.12: Output stiffness versus pivot point location. The four graphs (in the legend from top to bottom) represent the theoretical stiffness at $r-q_{2}=0$ rad, the simulated stiffness as found in the model, the measured stiffness from Figure 3.11 and the compensated stiffness. It can be seen that the simulated stiffness is approximated well by the compensated stiffness measurement.

linearly approximated stiffness of the actuator as a function of pivot position $q_{1}$. The dotted line represents the theoretical actuator output stiffness (3.6) for $r-q_{2}=0$, which is zero for $q_{1}=l$ and is infinite for $q_{1}=0$.

During the measurement, it has been observed that the setup suffers from a backlash effect due to the connection of input pulley and gearbox. At a certain externally applied output torque, the connection slips briefly before engaging again, as can be seen in the curve for $q_{1}=$ $0 \mathrm{~mm}$ at $\pm 6 \mathrm{~N}$. This effect has been circumvented by linearly approximating the lower portion of the curve in the third quadrant of Figure 3.11. This results in the compensated stiffness curve shown in Figure 3.12 (solid curve with open circular marks), which approximates the simulated output stiffness (dashed curve).

Unlike the theoretical stiffness, which increases to infinity for $q_{1} \rightarrow 0$, the simulated, measured, and compensated actuator output stiffnesses do not increase to infinity due to the parasitic timing belt compliance. The maximum stiffness is $948 \mathrm{Nm} / \mathrm{rad}$. Since the simulated stiffness closely matches the measured stiffness, it is observed that the model properly represents the stiffness property of the physical system.

The actuator work space is limited by physical constraints, i.e. the mechanical end-stops of the output and the limit on the maximum spring elongation. Deduced from Figure 3.11, an output stiffness versus output torque graph was found, as shown in Figure 3.13. The shaded area represents the reachable actuator settings, i.e. the actuator work space.

The measurement and identification results are summarized in Table 3.4. It can be shown that the design meets the initial design requirements shown in Table 3.1.

\subsection{Discussion}

In this chapter, the novel variable stiffness actuator vsaUT-II has been presented. The actuator innovates with the mechanism that is used to move the pivot along a lever arm.

The proposed mechanism uses a rolling transmission, which results in low friction. Fur- 


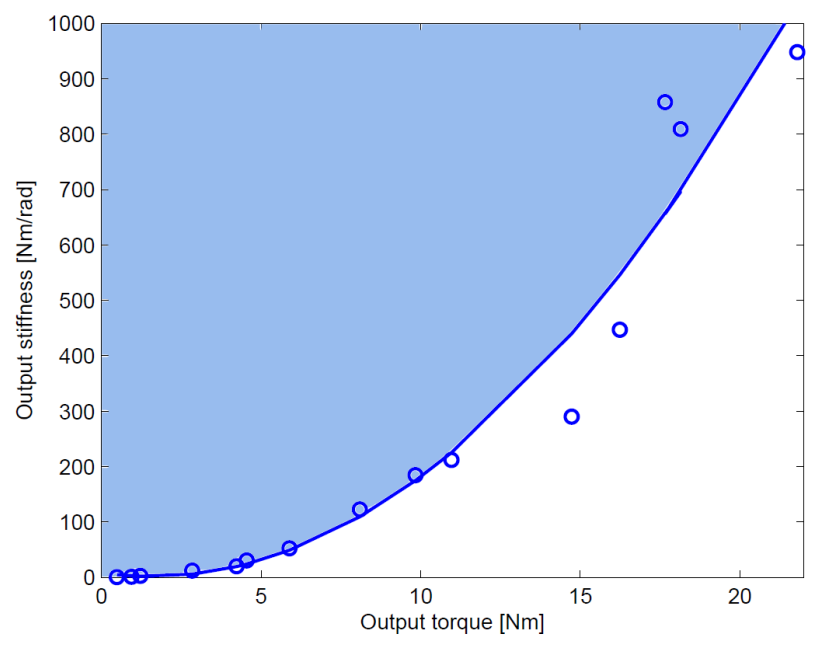

Figure 3.13: The vsaUT-II work space. The reachable output stiffness (shaded area) as a function of the output torque.

thermore, no linear guides are needed to constrain the pivot, since its motion is a straight line by design. Finally, a continuous rotation at the input results in a continuous harmonic motion of the pivot, as shown in (3.4). This means that for reversing the direction of the pivot at the end of its path, there is no need for reversing the input direction, if the lever arm would have length $l=D_{r}$. This means that the motor inertia does not have to be decellerated when the pivot needs to move in the opposite direction at the end of the lever arm and electrical peak currents are avoided.

However, some drawbacks can also be observed. For instance, the transmission from motor rotation to pivot translation is nonlinear, which might increase the control complexity. Moreover, play in the gears was observed during measurements, but this can be compensated.

\subsection{Conclusion}

In this chapter, the mechanical design, model and parameters identification of the variable stiffness actuator vsaUT-II was presented. In this actuation system, the apparent output stiffness and the output position can be varied independently thanks to the realization of a variable transmission between the internal elastic elements' force and the system output force. The variable transmission is implemented by means of a lever arm, whose effective length is given by the relative position of a pivot point along the lever. The innovation of the vsaUT-II is in how the motion of the pivot is realized, namely by means of a modified planetary gear mechanism. A lumped parameter model has been presented and the various system parameters have been identified. System measurements verified the model accuracy and the prototype performance. 
Table 3.4: Operating data for the vsaUT-II.

\begin{tabular}{|c|c|c|c|}
\hline \multicolumn{4}{|c|}{ Mechanical } \\
\hline 1 & Continuous Output Power & [W] & 69.8 \\
\hline 2 & Nominal Torque & {$[\mathrm{Nm}]$} & 21.8 \\
\hline 3 & Nominal Speed & {$[\mathrm{rad} / \mathrm{s}]$} & 3.2 \\
\hline 4 & Nom. Stiffness Variation Time: no load & [s] & 0.78 \\
\hline 5 & Nom. Stiffness Variation Time: nom. torque & [s] & 0.9 \\
\hline 6 & Peak (Maximum) Torque & [Nm] & 60 \\
\hline 7 & Maximum Speed & {$[\mathrm{rad} / \mathrm{s}]$} & 5.5 \\
\hline 8 & Maximum Stiffness ${ }^{1}$ & {$[\mathrm{Nm} / \mathrm{rad}]$} & 948 \\
\hline 9 & Minimum Stiffness & {$[\mathrm{Nm} / \mathrm{rad}]$} & 0.7 \\
\hline 10 & Maximum Elastic Energy & [J] & 0.19 \\
\hline 11 & Maximum Torque Hysteresis & [\%] & 12 \\
\hline 12 & Maximum Deflection: max. stiffness ${ }^{1}$ & {$\left[{ }^{\circ}\right]$} & 2 \\
\hline 13 & Maximum Deflection: min. stiffness & {$\left[{ }^{\circ}\right]$} & 40.1 \\
\hline 14 & Active Rotation Angle & {$\left[^{\circ}\right]$} & \pm 28.6 \\
\hline 15 & Angular Resolution & {$\left[^{\circ}\right]$} & 0.35 \\
\hline 16 & Weight & [kg] & 2.5 \\
\hline \multicolumn{4}{|c|}{ Electrical } \\
\hline 17 & Nominal Voltage & [V] & 24 \\
\hline 18 & Nominal Current & {$[\mathrm{A}]$} & 4 \\
\hline 19 & Maximum Current & [A] & 12 \\
\hline \multicolumn{4}{|c|}{ Control } \\
\hline 20 & Voltage Supply & [V] & 5 (USB), $7 . .12$ \\
\hline 21 & Nominal Current & [A] & 0.1 \\
\hline 22 & I/O Protocol & - & RS232 \\
\hline
\end{tabular}

${ }^{1}$ Maximum output stiffness and corresponding maximum deflection are a consequence of the finite structural stiffness of the actuator. 
CHAPTER 4

\section{Leaf-Spring Design: The VSM}

In this chapter, a novel variable stiffness mechanism is presented, which is capable of achieving an output stiffness with infinite range, and an unlimited output motion, i.e., the mechanism output is completely decoupled from the rotor motion, in the zero stiffness configuration. The mechanism makes use of leaf springs, which are engaged at different positions by means of two movable supports, to realize the variable output stiffness. The Euler-Bernoulli leaf spring model is derived and validated through experimental data. By shaping the leaf springs, it is shown that the stiffness characteristic of the mechanism can be changed to fulfill different application requirements. Alternative designs can achieve the same behavior with only one leaf spring and one movable support pin.

This chapter has been previously published as:

[62] S. Groothuis, R. Carloni, and S. Stramigioli, "A novel variable stiffness mechanism capable of an infinite stiffness range and unlimited decoupled output motion," Actuators, vol. 3, no. 2, pp. 107-123, 2014

Moreover, a patent application, included in Appendix D, with corresponding publication is done:

[63] S. Stramigioli and S. S. Groothuis, "WO2015102490 A1: Variable Stiffness Actuator (patent pending)," 2015 
$\mathrm{M}$ ANY different implementations of VSA mechanisms have been presented in literature (as already shown in Chapter 3), which are classified in three major groups in [64], i.e., the spring preload group (of which an antagonistic realization closely resembles a human joint) [41, 42, 43], the group in which the transmission between load and spring is changed $[47,50,48,49,38,39]$ and the group where the physical properties of the spring are changed $[44,65,46]$. Each design has its own characteristic properties: the spring preload designs rely on adjusting the preload of elastic elements, which allows them to store a considerable amount of energy, but requires them to put energy in the elastic elements to change the stiffness which may not be retrievable [54], while their stiffness range is finite; the variable transmission between load and spring designs require no energy to be put in the elastic elements to change the stiffness, but it is more difficult to store a considerable amount of energy. Infinite stiffness range is, however, possible; the designs where the physical properties of the spring are changed, also require no energy to be put into the elastic elements to change their stiffness and a considerable amount of energy can be stored in the elastic elements, although an infinite stiffness range within this class has not yet been shown in literature.

In this chapter, a novel design of a variable stiffness mechanism (VSM) is presented, which fits in the group in which the transmission between load and spring is changed. The novelty lies in the property that when the mechanism is in its zero stiffness configuration, the output is completely decoupled from the input (the rotor) and an unlimited output motion is then possible (unconstrained, infinite rotation). Therefore, the output behavior will only be a result of the dynamics of the output load. Moreover, the actuator is capable of achieving an infinite stiffness range (from truly zero stiffness to infinite stiffness, i.e., the parasitic mechanical stiffness), which was not shown before using leaf springs.

First, the conceptual design is presented in Section 4.1. Section 4.2 elaborates on the mechanism model and Section 4.3 shows the experiments performed on a setup very similar to the prototype mechanism. The chapter is concluded with Section 4.4.

\subsection{Conceptual Design}

This section elaborates on the conceptual design, showing the working principle of the VSM and the key design features that enable an infinite stiffness range and completely decoupled and infinite output motion from the input.

\subsubsection{The variable stiffness mechanism and its working principle}

The novel VSM, shown in Figure 4.1, consists of a stator, a rotor and an output. In this VSM, the compliance is created between the rotor and the output by using two leaf springs that connect to the output at their ends and connect to the rotor via support pins, fixed on the gears of a hypocycloid gearing mechanism. These pins can move in between the leaf springs in a straight line, imposed by the hypocycloid gearing mechanism, allowing variable compliance in between the rotor and the output. The two planet gears are connected to and rotated by the planet carrier.

The working principle of the concept (of only one leaf spring) is shown in Figure 4.2. The principle is based on the group in which the transmission between load and spring is changed. A force couple $F$ is applied on the undeflected leaf spring (in grey), which causes it to deflect (in black). The location of the movable supports (with coordinate $x_{0}$ ) influences the deflection shape of the entire leaf spring. For an increasing $x_{0}$, the transmission between load and spring is changed and, therefore, the perceived stiffness at the force application points is increasing. When the supports are located at both ends of the leaf spring, an infinite stiffness 


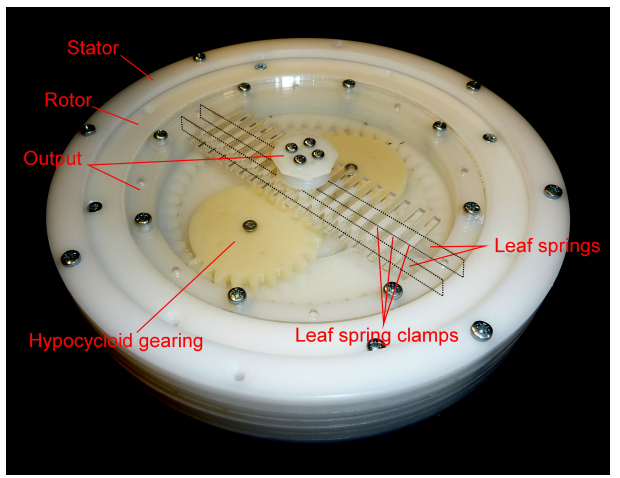

(a) Top view.

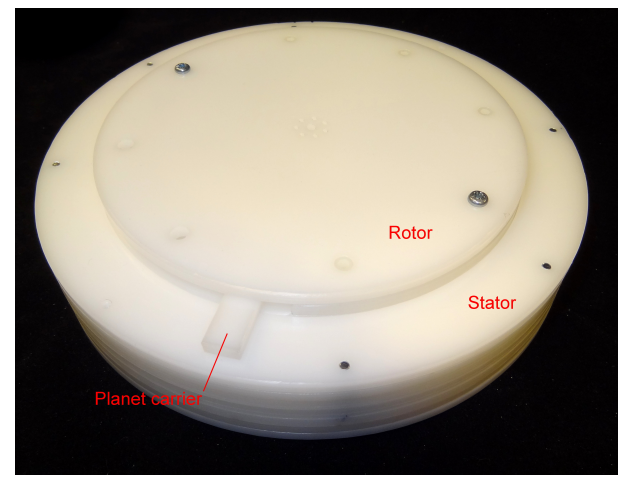

(b) Bottom view.

Figure 4.1: The novel variable stiffness mechanism creates compliance between the rotor and output by means of leaf springs and it is made variable by means of support pins that can be moved in between the leaf springs by using a hypocycloid gearing mechanism.

is felt. When the supports are joined together in the middle of the leaf spring, i.e., $x_{0}=0$, a zero stiffness is felt and, moreover, the leaf spring is free to rotate about its center due to force couple $F$. In the VSM, this means that the output is completely decoupled from the rotor and that the output only shows dynamics imposed by the load. Note that the active length is always the entire leaf spring, so the entire leaf spring determines the perceived stiffness.

Note that the device presented here is not yet an actuator in the sense that it has motors and can deliver power to a load. Currently, it is a conceptual mechanism to which actuators can be connected.
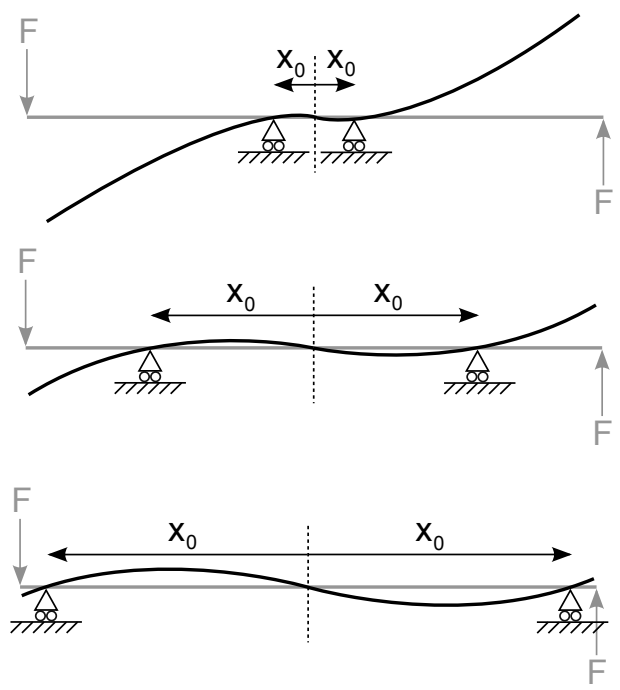

Figure 4.2: Working principle of the variable stiffness mechanism. The deflection shape of the leaf spring is varied by changing the location of the supports, and, therefore, the perceived stiffness at the location of the force application points is changed. 


\subsubsection{Support pins}

The support pins, at location $x_{0}$, have a special shape (the designed shape ${ }^{1}$ is shown in Figure 4.3) such that they can join together in the center of the VSM to form a perfect cylinder, such that the output is decoupled from the input. This means that the output can rotate infinitely, independent of the input. Note that when the output is rotated exactly perpendicular to the imaginary line along which the supports move, a singularity occurs because of the leaf springs constraining the supports to move apart. Since the rotor movement is also independent from the input, a controller should keep track of the output movement during free rotation and adjust the rotor position accordingly to prevent this singularity from occurring. When moving apart, the shape of the support pins ensures that the space between the two leaf springs is completely filled, leaving no room for play, at any location along the leaf springs, which is made explicit in Figure 4.4.

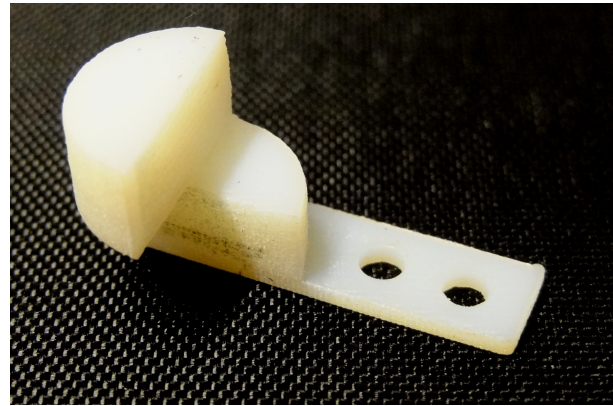

(a)

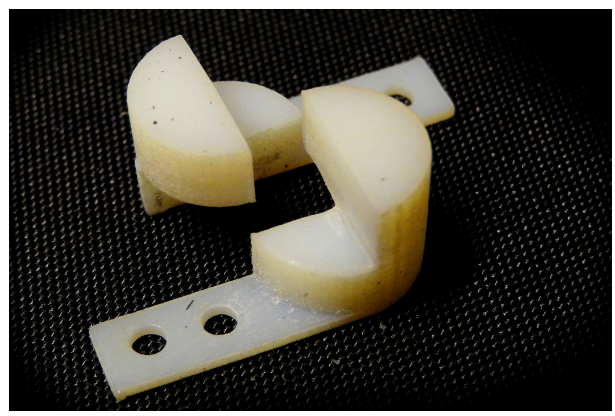

(c)

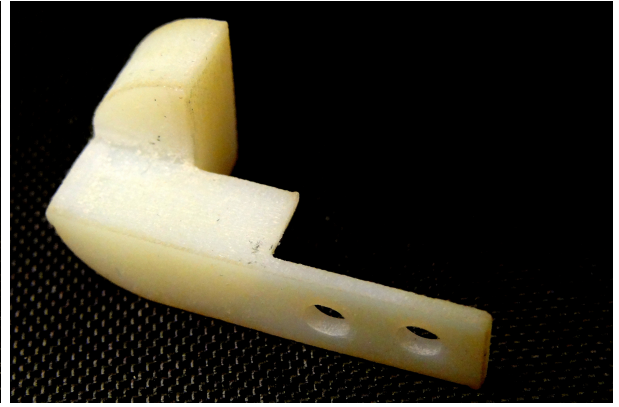

(b)

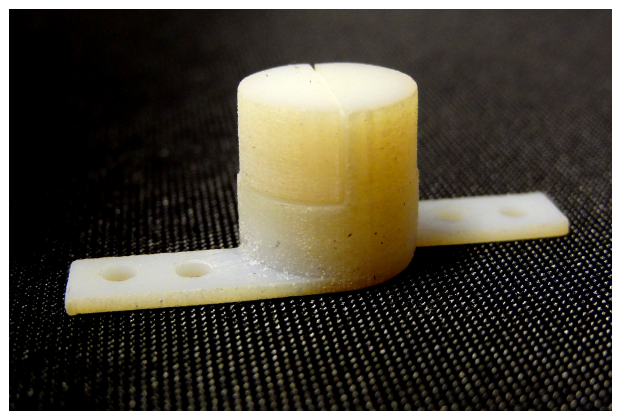

(d)

Figure 4.3: The pin used as one of the two supports for the leaf spring.

\subsubsection{Hypocycloid gearing mechanism}

The supports pins should be moved in a straight line along the leaf spring, which is achieved using a hypocycloid gearing mechanism (similar to a planetary gearing system, previously proposed in [39]) and shown in Figure 4.5. It consists of one ring gear and two planet gears and the supports are fixed to the planet gears. The ring gear is fixed to the rotor and the planet gears are fixed to and rotated by the planet carrier. Because the pitch diameter of the planet gears is half the pitch diameter of the ring gear, a point on the planet gear pitch diameter tracks a straight line. Note that half of the planet gear teeth should be removed to allow the

\footnotetext{
${ }^{1}$ Note that other pin shapes similar to this are possible, such as a helical shape.
} 


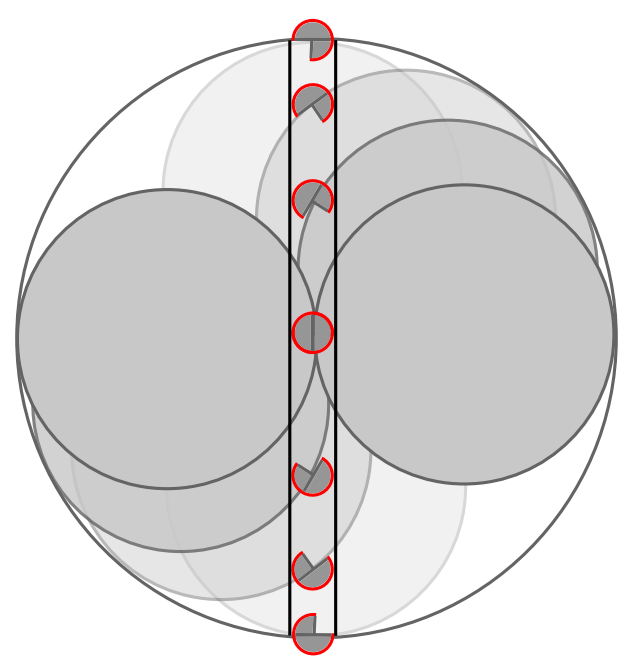

Figure 4.4: Schematic representation of the motion of the supports in between the leaf springs. Contact with both leaf springs is always ensured because of the pin shape (shown in red).

two planets to rotate. In the proposed VSA mechanism, there are two leaf springs placed at a small distance from each other in between which the supports can move. To ensure the leaf springs are held together at the same distance and deflect as if they are one leaf spring, they are held together by small clamps.

\subsection{Modeling}

This section elaborates on the VSM modeling. A leaf spring was modeled as a beam to analyse its deflection for specific support locations and applied forces. Although the mechanism consists of two leaf springs, only one leaf spring is modeled, since an otherwise highly complex model would likely result from the modeling of the coupling between the leaf springs by the clamps. A finite element model (FEM) of the support pin was used to analyze the stress on the pins as a result of the actuator torque.

\subsubsection{Leaf spring}

The leaf spring is modeled as a doubly supported, doubly overhanging rectangular EulerBernoulli beam with a force couple on the ends due to the mechanism output torque (refer to Figure 4.2). A Euler-Bernoulli model of this beam is justified versus the use of, for example, the Timoshenko beam model taking shear deformation into account, since the leaf spring is relatively thin compared to its other dimensions. Hence, assuming that a cross-section is always perpendicular to the neutral axis is justified.

The geometry of the beam and the coordinate frame that is used, which is placed in the center of the beam, is shown in Figure 4.6. The beam deflection in the $z$-direction is a function of the $x$-coordinate.

The general linear Euler-Bernoulli beam equation is given by:

$$
\frac{\mathrm{d}^{2}}{\mathrm{~d} x^{2}}\left(B(x) \frac{\mathrm{d}^{2}}{\mathrm{~d} x^{2}}(v(x))\right)=q(x),
$$




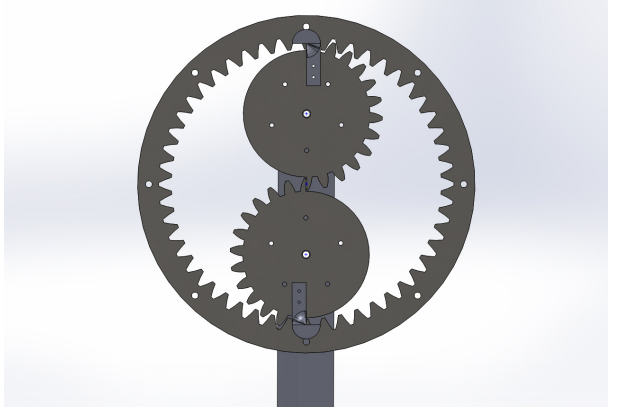

(a)

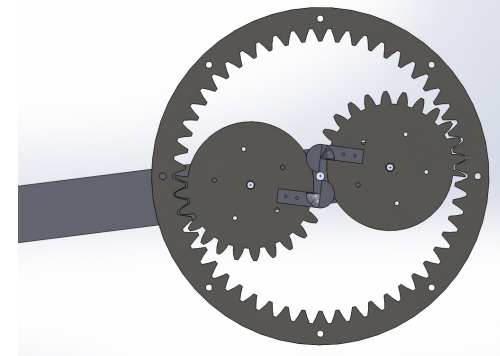

(c)

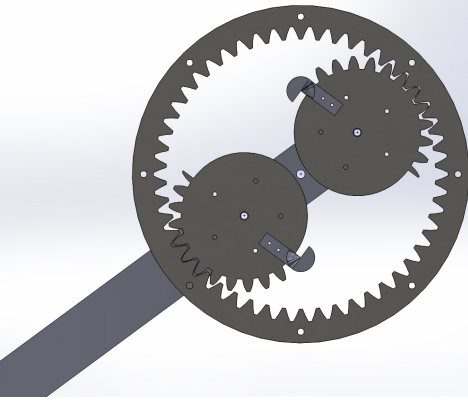

(b)

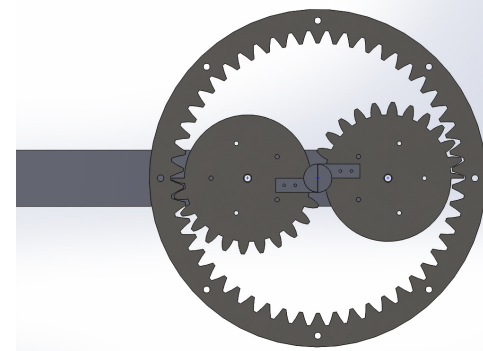

(d)

Figure 4.5: Hypocycloid gearing mechanism used to move the supports in a straight line along the leaf spring.

where the term $B(x):=E I_{x x}(x)$ is a function of the Young's modulus $E^{2}$ and the area moment of inertia $I_{x x}, v(x)$ is the beam deflection at coordinate $x$ and $q(x)$ is the loading profile on the beam.

Expanding the terms, this equation can be given by:

$$
\frac{\mathrm{d}^{2}}{\mathrm{~d} x^{2}} E I_{x x}(x) \frac{\mathrm{d}^{2}}{\mathrm{~d} x^{2}} v(x)+2 \frac{\mathrm{d}}{\mathrm{d} x} E I_{x x}(x) \frac{\mathrm{d}^{3}}{\mathrm{~d} x^{3}} v(x)+E I_{x x}(x) \frac{\mathrm{d}^{4}}{\mathrm{~d} x^{4}} v(x)=q(x) .
$$

This is a fourth order, first degree, ordinary differential equation with variable coefficients.

For a uniform beam, i.e., a beam with constant geometry $E I_{x x}(x) \rightarrow E I_{x x}$, the first two terms are zero and the equation becomes:

$$
E I_{x x} \frac{\mathrm{d}^{4}}{\mathrm{~d} x^{4}} v(x)=q(x)
$$

This is a fourth order, first degree, ordinary differential equation with constant coefficients.

The beam deflection can be solved by separately taking into account the individual loading segments and choosing proper boundary conditions to form a continuous solution. However, the loading profile can also be specified in a way such that it is valid over the complete beam length and solving it for the complete beam at once. The loading on the leaf spring consists of four forces, as shown in Figure 4.7. The loading $q(x)$ can be given as:

\footnotetext{
${ }^{2}$ It is assumed that the leaf spring has uniform density, i.e., the Young's modulus $E$ is constant over the material.
} 


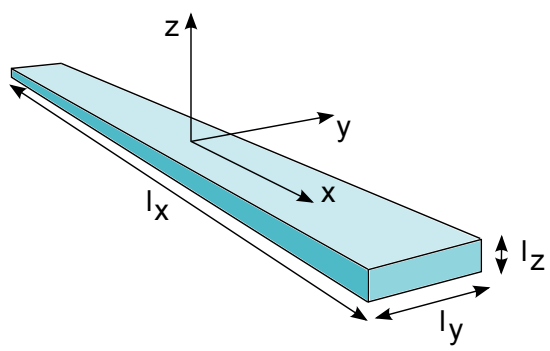

Figure 4.6: Geometry and coordinate usage of a generic beam.

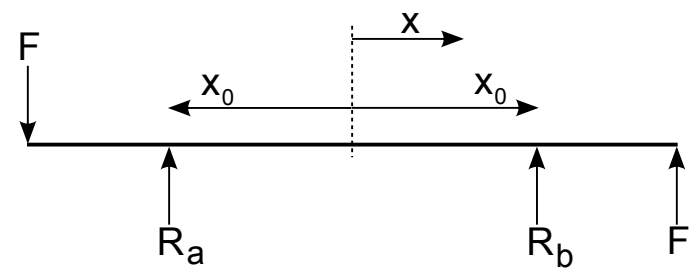

Figure 4.7: Force profile on the leaf spring, consisting of four forces: two applied forces $F$ and two reaction forces on the support pins $R_{a}$ and $R_{b}$.

$$
q(x):=-F \cdot \delta\left(x+\frac{1}{2} l_{x}\right)+R_{a} \cdot \delta\left(x+x_{0}\right)+R_{b} \cdot \delta\left(x-x_{0}\right)+F \cdot \delta\left(x-\frac{1}{2} l_{x}\right),
$$

where $F$ is the force on the ends of the leaf spring due to the output torque, $R_{a}$ and $R_{b}=-R_{a}$ are the reaction forces at the supports and $\delta(x-\alpha)$ is the Dirac pulse located at $x=\alpha$.

The fourth order differential equation of (4.1) needs four boundary conditions to solve for the deflection $v(x)$. Two boundary conditions are given at the support locations, since there the deflection is zero. Figure 4.8 gives the other two boundary conditions, namely the slope at the ends of the leaf springs should be coincident with the center of the mechanism. The boundary condition equations are given by:

$$
\begin{aligned}
& v\left(x_{0}\right)=0 \\
& v\left(-x_{0}\right)=0 \\
&\left.\frac{\mathrm{d}}{\mathrm{d} x} v(x)\right|_{x=\frac{1}{2} l_{x}}:=\phi\left(\frac{1}{2} l_{x}\right)=\frac{v\left(\frac{1}{2} l_{x}\right)}{\frac{1}{2} l_{x}} \\
&\left.\frac{\mathrm{d}}{\mathrm{d} x} v(x)\right|_{x=-\frac{1}{2} l_{x}}:=\phi\left(-\frac{1}{2} l_{x}\right)=\frac{v\left(\frac{1}{2} l_{x}\right)}{\frac{1}{2} l_{x}}
\end{aligned}
$$

The leaf spring bends in the $z$-direction, so the area moment of inertia $I_{x x}$ for a uniform beam is given by:

$$
I_{x x}:=\frac{l_{y} l_{z}^{3}}{12} .
$$

The Young's modulus $E$ depends on the material that is used. In this mechanism PETG copolyester with a Young's modulus of $1.9 \mathrm{GPa}$ was used.

When solving the equation in (4.1) together with the boundary conditions of (4.2) for a uniform beam, with lengths $l_{x}=100 \mathrm{~mm}, l_{y}=8 \mathrm{~mm}$ and $l_{z}=1 \mathrm{~mm}$, the beam shape for different settings of the support location with coordinate $x_{0}$ is shown in Figure 4.9. Indeed, 


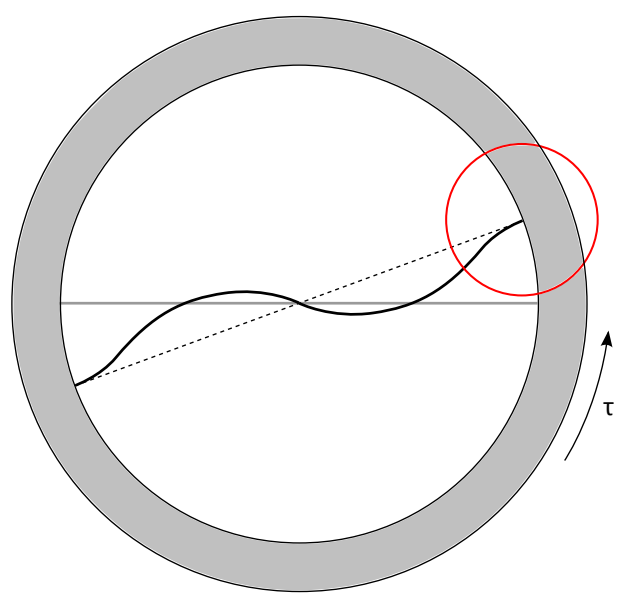

Figure 4.8: The leaf spring (thick solid line) is fixed to the output of the VSA mechanism (grey ring), which causes the leaf spring slope at its ends (dashed line) to be coincident with the mechanism center and perpendicular to the tangent of the output ring.

for increasing $x_{0}$ the beam deflection decreases at a constant force $F$, and, therefore, the perceived stiffness increases. Note the particular beam shape at the ends, caused by clamping the leaf spring in the output.

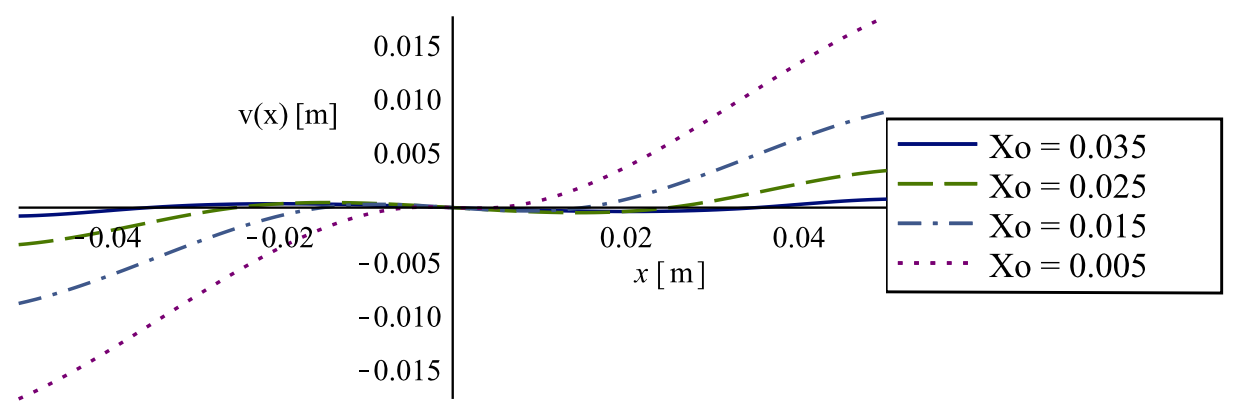

Figure 4.9: Beam shape for several settings of the support location $x_{0}$ under a constant applied force $F$. Note the particular beam shape near the ends, caused by clamping the leaf spring in the mechanism output.

When the leaf spring is not uniform, but either variable over length $l_{y}$ or $l_{z}$ as a function of coordinate $x, I_{x x}$ becomes a function of $x$. This affects the stiffness of the beam, which is a desirable feature of the proposed mechanism, since a different stiffness characteristic can be very easily achieved by only adjusting the shape of the leaf spring. When assuming that only length $l_{y}$ is variable over coordinate $x$, which is realized more easily than a variable $l_{z}$, the area moment of inertia becomes:

$$
I_{x x}(x):=\frac{l_{y}(x) l_{z}^{3}}{12} .
$$

In Section 4.3, three different leaf spring shapes are measured to make the shaping of the stiffness characteristic explicit. Equations (4.1) and (4.2) of these non-uniform beams were solved numerically and those results are plotted together with the measurements in Section 4.3.

Note that the model presented here is based on linear beam theory and, therefore, the model is less accurate for larger deflections. 


\subsubsection{Support pins}

The support pins should have a special shape to allow two of those pins to join together, while still ensuring that the complete space between two leaf springs in the mechanism is filled, such that there is no play between the output and the rotor. The support pins should also be able to withstand the forces as a result of force $F$ on the leaf spring.

The force on a support pin is equal to $F$ when a support is located at $x_{0}=\frac{1}{2} l_{x}$ and increases when $x_{0}$ decreases. The maximum support force occurs for $0<x_{0} \ll \epsilon$, with $\epsilon$ infinitely small, i.e., at a location infinitely close to zero, but not equal to zero. For a constant output force $F$, this would result in an impossibly large deflection of the leaf spring. Therefore, to calculate the maximum support force, its deflection needs to be limited. Figure 4.10 shows the force in one of the supports, for a constant applied maximum force $F=F_{\max }$ and for an applied force $F$ that causes a certain constant maximum leaf spring deflection $F=F_{v_{\max }}$. For a maximum force $F_{\max }=200 \mathrm{~N}$ (corresponding to an arbitrary maximum output torque of $\tau=10 \mathrm{Nm}$ ), and a maximum allowable leaf spring deflection $v_{\max }=\frac{1}{4} l_{x}$, the maximum support force is calculated to be $R_{a \max }=238.4 \mathrm{~N}$ at $x_{0}=42.0 \mathrm{~mm}$.

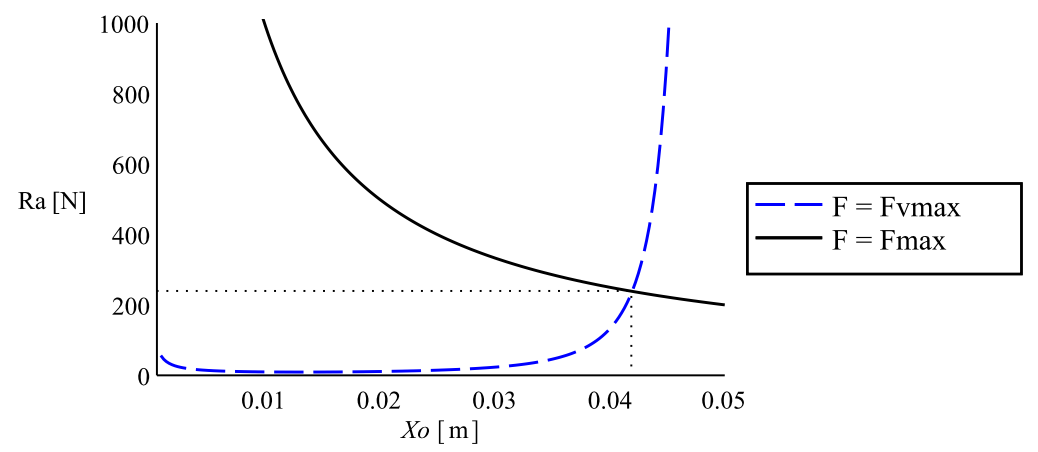

Figure 4.10: Support force $R_{a}$ as a function of support location $x_{0}$. Two plots are shown: $F=F_{\max }$ shows the support force if always a force $F=F_{\max }$ is allowed to be applied on the leaf spring and $F=F_{v_{\max }}$ shows the support force when a certain force $F$ is applied with which a certain maximum deflection $v(x)=v_{\max }$ is achieved. Their crossing is the maximum possible support force $R_{a \max }$.

The maximum force $F_{\max }$ on the support pin occurs at a certain support location $x_{0}$ and, therefore, under a certain angle defined by the motion of the hypocycloid gearing mechanism. It is calculated that this angle for $x_{0}=42.0 \mathrm{~mm}$ is $\theta_{\max }=32.96^{\circ}$. That force is applied on a finite element model of the support pin, refer to Figure 4.11, to obtain the stress distribution inside the pin. Using regular alloy steel with a Young's modulus of $210 \mathrm{GPa}$ and a yield strength of $620 \cdot 10^{6} \mathrm{~N} / \mathrm{m}^{2}$, the safety factor using this particular shape is 3.10 .

\subsubsection{Hypocycloid gearing mechanism}

Coordinate $x_{0}$ is a function of the planet carrier angle in the hypocycloid gearing mechanism (the angle that the bar to which the two planet gears are fixed makes with respect to the ring gear, see Figure 4.5), $\theta_{p c}$, and the pitch diameter of the planet gear, $D_{p}$, and is given by $x_{0}=D_{p} \sin \left(\theta_{p c}\right)$. Here, it is assumed that the bottom right figure of Figure 4.5 represents a planet carrier angle of $\theta_{p c}=0$ rad and the top left figure represents an angle of $\theta_{p c}=\frac{1}{2} \pi$ rad. $D_{p}$ is $50 \mathrm{~mm}$ in this setup. Readers are referred to [39] for the full kinematic analysis. 


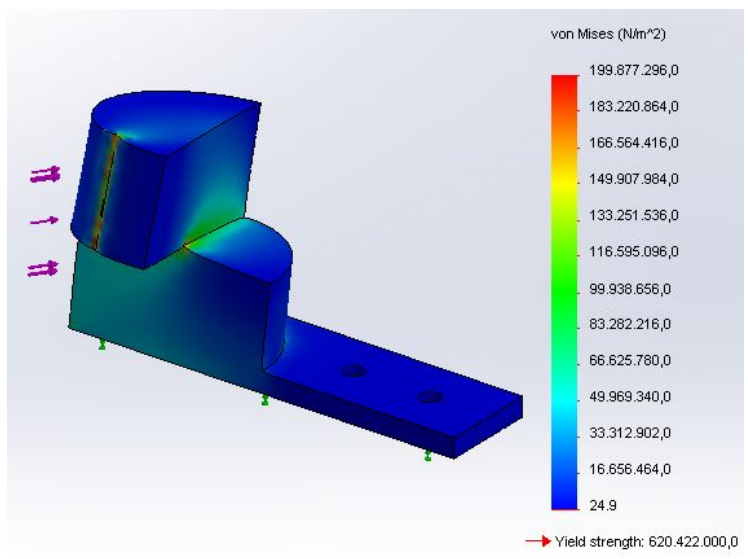

Figure 4.11: Stress analysis of the support pin fixed to a gear of the hypocycloid gearing mechanism. The applied loading is given by the maximum force on the pin under a certain angle.

\subsection{Experiments}

Experiments have been performed to measure the stiffness characteristic of the proposed variable stiffness mechanism. As stated before, the stiffness characteristic that is a function of the support location $x_{0}$ can be changed by shaping the leaf springs. Three different PETG leaf spring shapes were used during the measurements, namely a uniform shape, a negative parabola cutout and a positive parabola cutout, as is shown in Figure 4.12. In 1, the height is $8 \mathrm{~mm}$, in 2 and 3, the height is between 4 and $8 \mathrm{~mm}$. The expectation is that for support locations close to the center, i.e., for small $x_{0}$, the positive parabola stiffness is larger than the negative parabola stiffness, but for support locations further from the center, the positive parabola stiffness increases less steep compared to the negative parabola stiffness. The uniform leaf spring should have the highest overall stiffness, since it has the largest dimensions over its full length. The experimental setup of Figure 4.13 was used to measure the stiffness

1

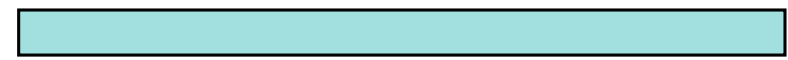

2

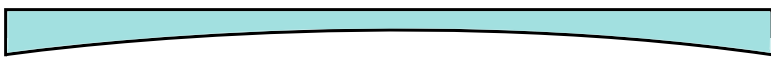

3

Figure 4.12: Three different leaf spring shapes, shown in the $x-y$-plane. Leaf spring 1 is a uniform beam and leaf spring 2 and 3 are beams with a negative and positive parabola cutout, respectively.

characteristic of the VSM. It is similar to the VSM itself, but now the output has a handle with which it can be rotated. On the bottom an ATI Mini40 force/torque sensor (SI-80-4 calibration) is fixed between the rotor and the fixed world, and, moreover, an AMS5048A magnetic absolute angular encoder with a resolution of 14 bits is placed on top of the setup to measure the output angle. A measurement is done by placing the correct leaf springs, setting the correct support location and rotating the output once from its neutral position in the positive direction, back to its neutral position, in the negative direction and back to its neutral position. In this way, any non-symmetrical behavior and the possible hysteresis effect are 
made explicit. The two sensors are interfaced with Matlab Simulink (The Mathworks, Inc., Natick, MA, USA), where the force/torque and angle data are acquired simultaneously and processed further. A deflection/torque diagram can be obtained directly from such measurement for certain $x_{0}$-settings and a stiffness/support location diagram is obtained by a linear least squares approximation to this data. The slope is then the compliance and its reciprocal is the stiffness at that $x_{0}$-setting.

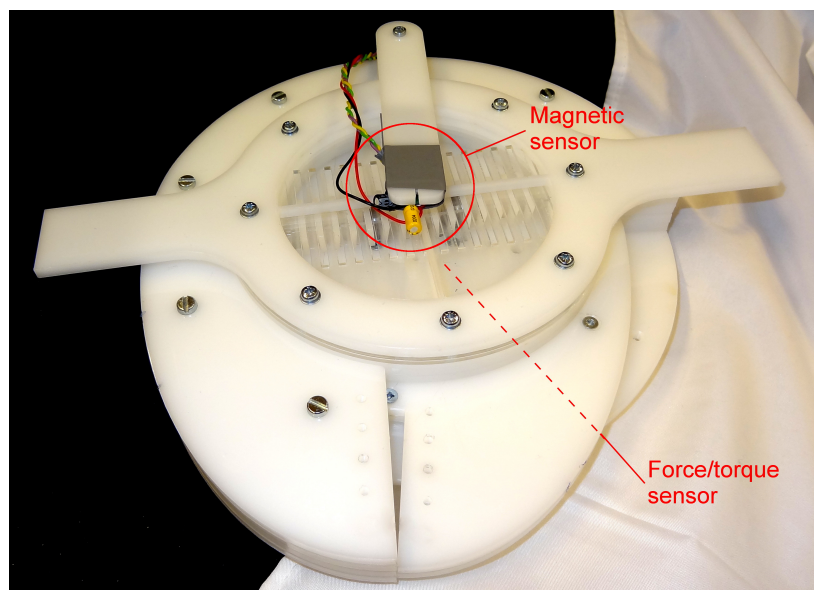

(a)

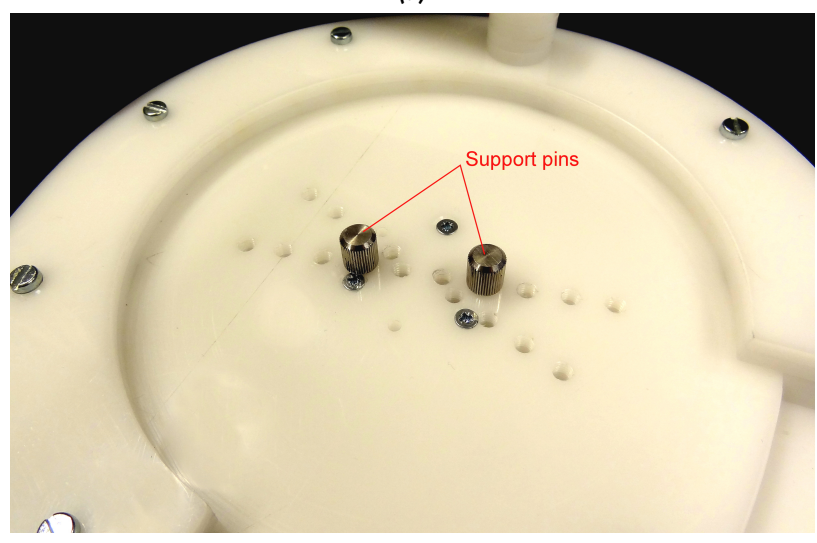

(b)

Figure 4.13: The experimental setup used to measure the stiffness characteristic of the variable stiffness mechanism. A magnetic sensor is placed over the device to measure the output rotation, while a force/torque sensor is placed under the device to measure the applied torque. The support pins can be placed at predefined locations.

Since the model assumes only one leaf spring, also one leaf spring was first measured in the setup. Since the mechanism is designed for using a double leaf spring, a single leaf spring was fixed to the output and placed in between the two supports with a slight pretension, such that it is possible to measure the torque/deflection characteristic in one direction. See Figure 4.15 for (part of) the measurement results. Measurement data is shown for five different support location settings. The simulated torque/deflection data is shown with dashed light grey lines. A dotted line in the same color as the measurement data indicates the linear least squares approximation to the data. Figure 4.16 shows the support location/stiffness plots derived from Figure 4.15. Again the dashed light grey line is the simulated stiffness and the solid blue 
line is the measured stiffness. It can be seen that the experiments show the same characteristic as the simulations and the experiments thereby validate the model in Section 4.2.

Using the same measurement procedure, the situation for the regular configuration of two leaf springs was measured and these measurements were again used to obtain a support location/stiffness plot. Modeling the effect of a double leaf spring constrained by clamps would likely results in a highly complex model, so this effect was empirically tested by normalizing the double leaf spring stiffness at each support location to the stiffness obtained when using only one leaf spring, for every leaf spring shape. This results in Figure 4.14. These normalized stiffnesses are approximated by a linear regression for increasing support location $x_{0}$, averaged over the three leaf spring shapes, shown in dashed light grey. With this effect, the model data is adapted to incorporate the coupling to a second leaf spring.

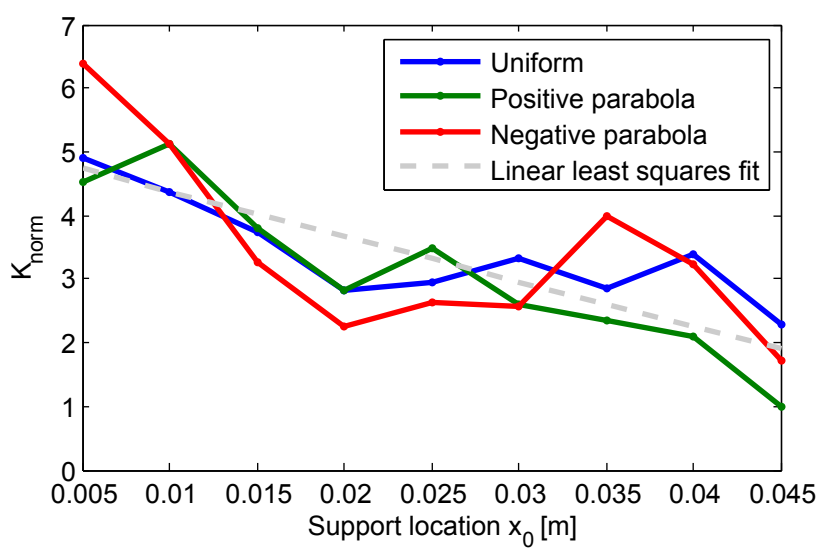

Figure 4.14: Normalized stiffness derived from the double leaf spring measurement, normalized to the single leaf spring measurement, which shows the effect of the double leaf spring and clamps. This effect is approximated by a linear regression for increasing support location $x_{0}$, averaged over the three leaf spring shapes.

Figure 4.17 shows (part of) the torque/deflection plots using a double leaf spring, presented in Section 4.1. Again, the dashed light grey lines are the simulated data, that are now adapted to incorporate the coupling of the second leaf spring, and the dotted lines in the same color as the measurement data are the linear least squares approximations. These approximations are used to calculate the stiffness using a particular leaf spring shape, which is shown in Figure 4.18. The dashed line is the adapted simulation data and it is closely approximated by the experimental data.

In Figure 4.15 and 4.17, hysteresis can be seen. This is due to the realization of the test setup, which has friction between the rotatable output and stator, and stiction of the leaf spring in the groove that connects it to the output to allow shortening of the leaf springs under deflection. Hence, this is not an intrinsic property of the mechanism. 


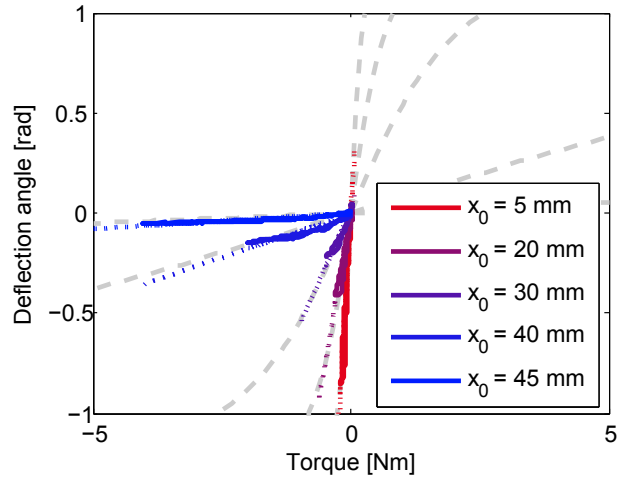

(a) Uniform

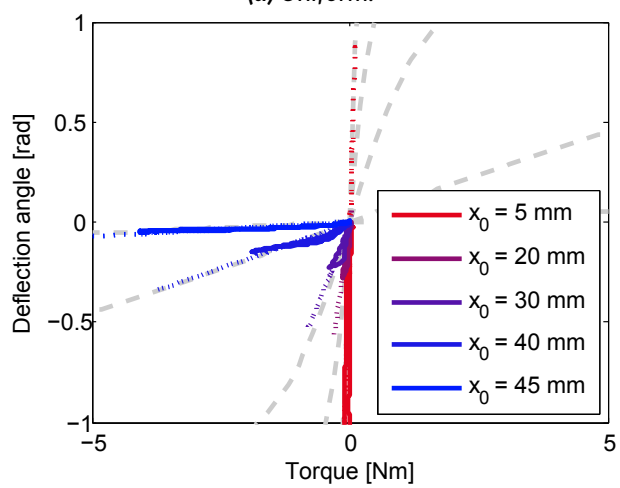

(b) Negative parabola.

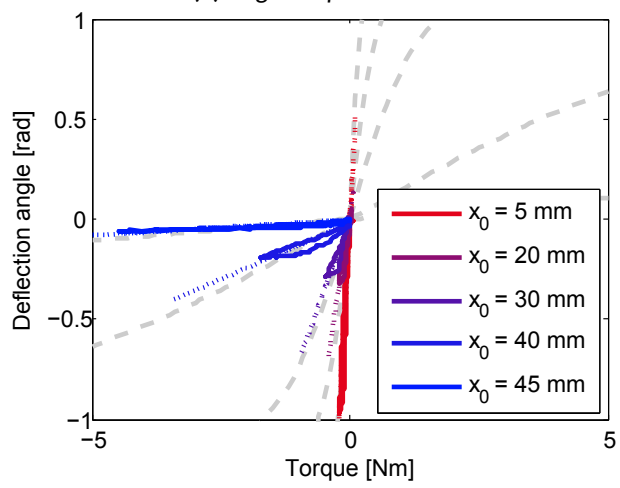

(c) Positive parabola.

Figure 4.15: Deflection/torque measurement results using a single leaf spring (for clarity only half of the measurements are shown). The dashed line represents the simulated deflection/torque data and the dotted line is the linear least squares approximation to the deflection/torque data.

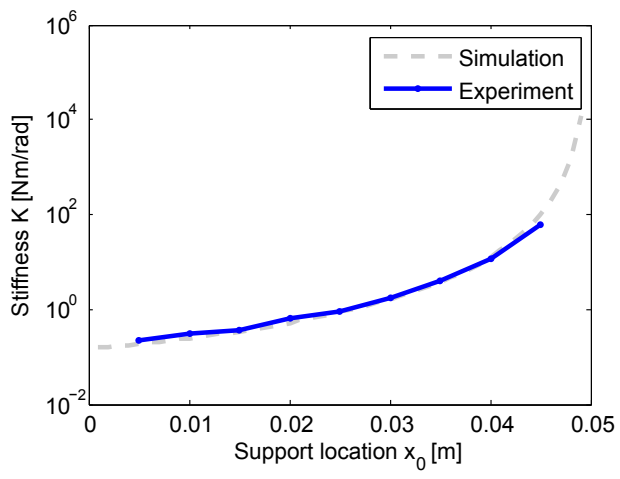

(a) Uniform.

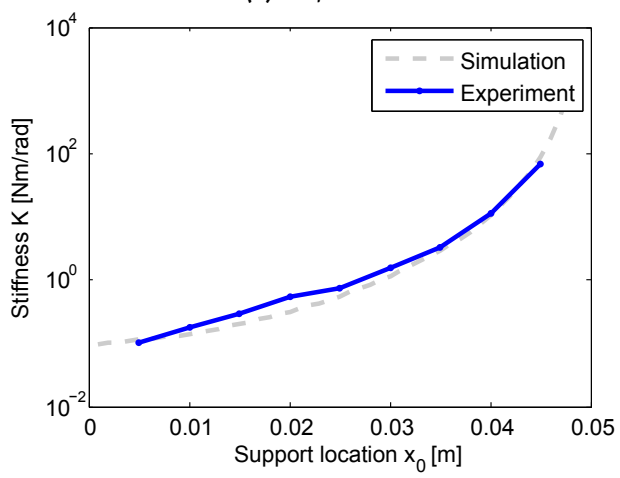

(b) Negative parabola.

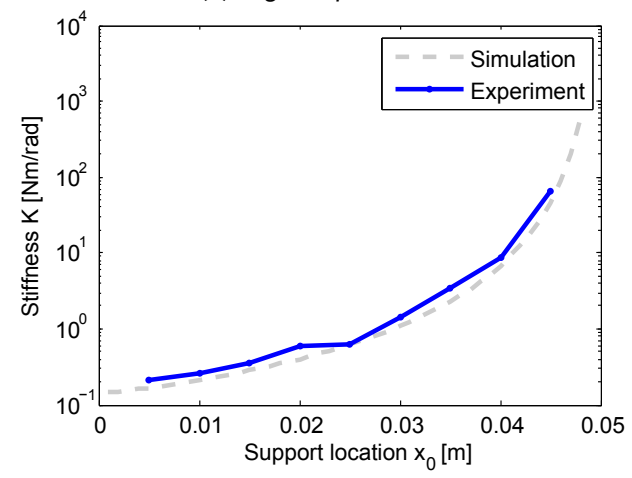

(c) Positive parabola.

Figure 4.16: Stiffness/support location plots for a single leaf spring based on a linear least squares approximation to the deflection/torque data. 


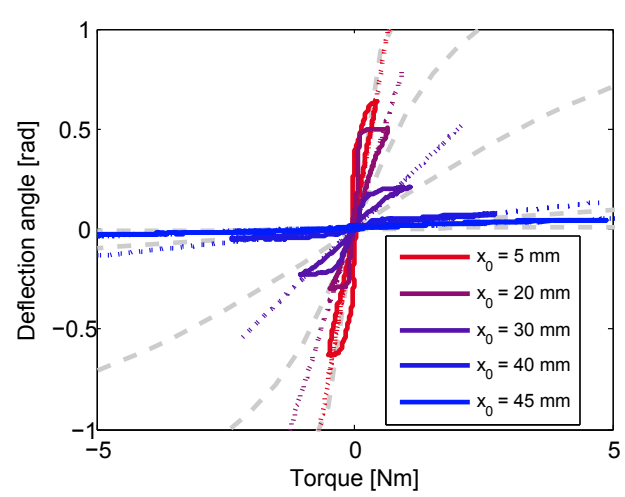

(a) Uniform.

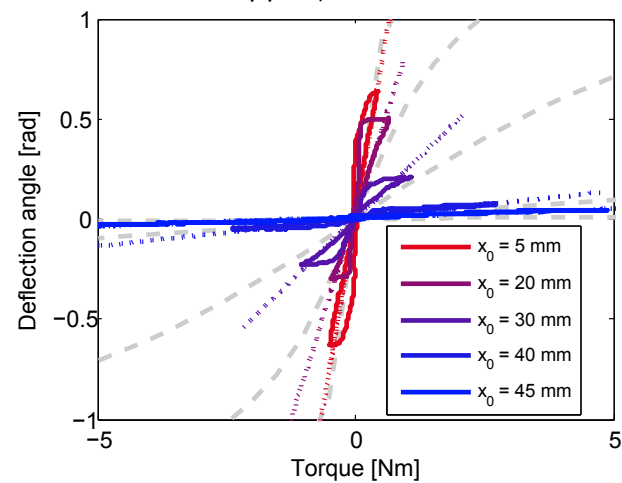

(b) Negative parabola.

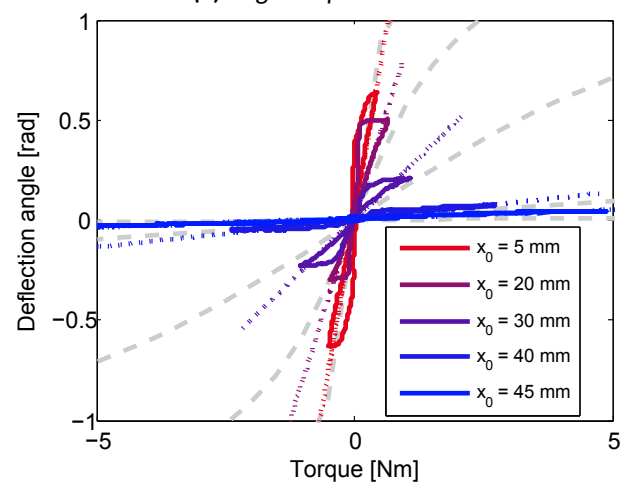

(c) Positive parabola.

Figure 4.17: Deflection/torque measurement results using a double leaf spring (for clarity only half of the measurements are shown). The dashed line represents the simulated deflection/torque data and the dotted line is the linear least squares approximation to the deflection/torque data.

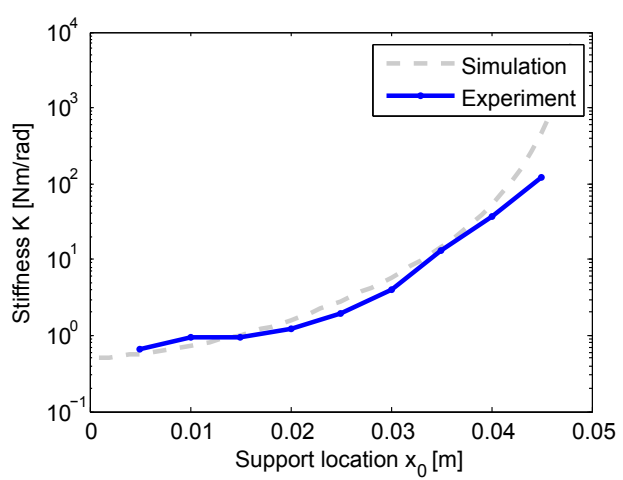

(a) Uniform.

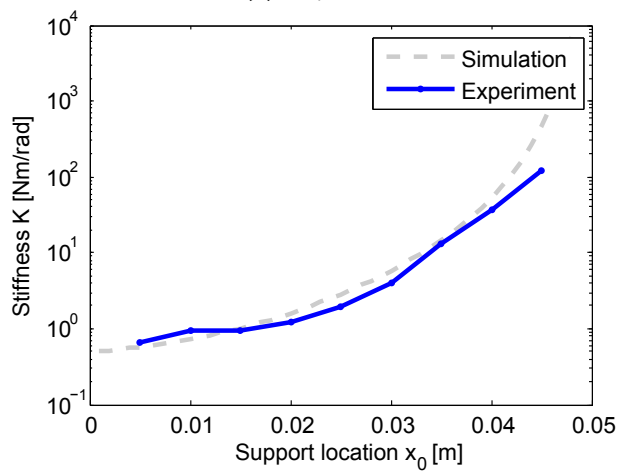

(b) Negative parabola.

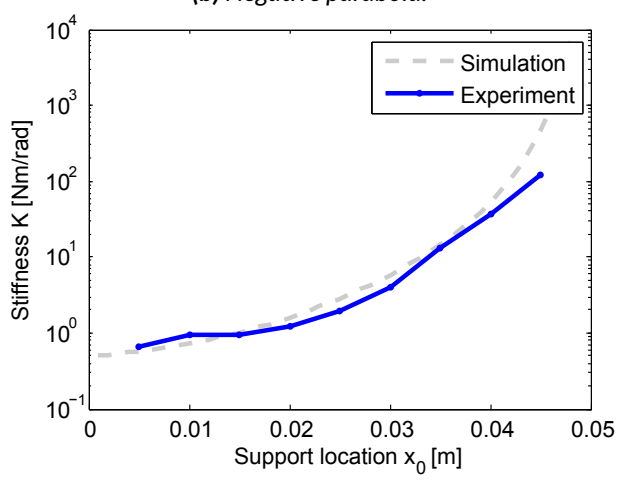

(c) Positive parabola.

Figure 4.18: Stiffness/support location plots for a double leaf spring based on a linear least squares approximation to the deflection/torque data. The simulated line is adapted using the measured effect of the double leaf spring and the clamps with respect to a single leaf spring. 


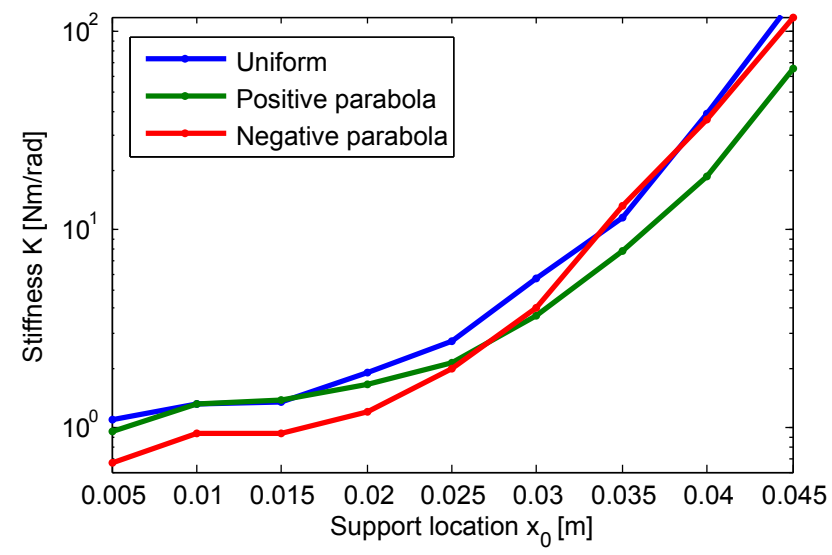

Figure 4.19: Stiffness plots for the three different leaf spring shapes, making the possibility of shaping the stiffness characteristic of the VSM explicit.

Figure 4.19 overlays the three stiffness plots of Figure 4.18 to show the ability that the stiffness characteristic of the VSM can be changed easily by shaping the leaf springs. Although for these shapes the effect is not that profound, the stiffness shaping possibility is obviously seen when comparing the two non-uniform leaf springs: the positive parabola starts with a higher stiffness than the negative parabola, but increases less steeply such that the negative parabola stiffness exceeds the positive parabola stiffness at $x_{0}=27 \mathrm{~mm}$. Moreover, the positive parabola stiffness for small support locations is the same as the stiffness of the uniform leaf spring, since the positive parabola has almost equal dimensions for small support locations. This is indeed according to the expectation. More leaf spring shapes can be explored, to achieve, for instance, a stiffness characteristic that is linear over a maximal interval.

\subsection{Conclusions}

In this chapter, a novel variable stiffness mechanism has been presented, which is capable of an infinite stiffness range not before encountered in this class of variable stiffness realizations, and completely decoupled unlimited output motion with respect to the rotor for safe passive behavior. An important feature of this mechanism is the ability to easily change the stiffness characteristic by shaping the leaf springs. A Euler-Bernoulli beam model is proposed to model one of the two leaf springs that are present in the mechanism. Experiments using PETG copolyester show the validity of this model for single leaf springs of various shapes. The stiffness measurement using the double leaf spring is normalized to the single leaf spring stiffness measurement, to measure the effect of coupling a leaf spring by clamps to another leaf spring. This effect is approximated and used to adapt the model data to correspond to the double leaf spring measurements. In this way, a close approximation of the measurements to the model data is achieved. The result of shaping the leaf springs is shown and agrees with the expectation.

\subsection{Outlook}

A different realization of the same concept might be explored in future research, possibly one that uses only one leaf spring and one circular support with a groove, in which the leafspring 
is placed, that is mounted with a bearing on the planet gear. Also, a fixed support with two quarter cylinder cut-outs may be possible. Note that these different realizations do not change the properties of the mechanism and it should be investigated whether these realizations have advantages over the presented mechanism. See Figure 4.20 for a schematic representation.

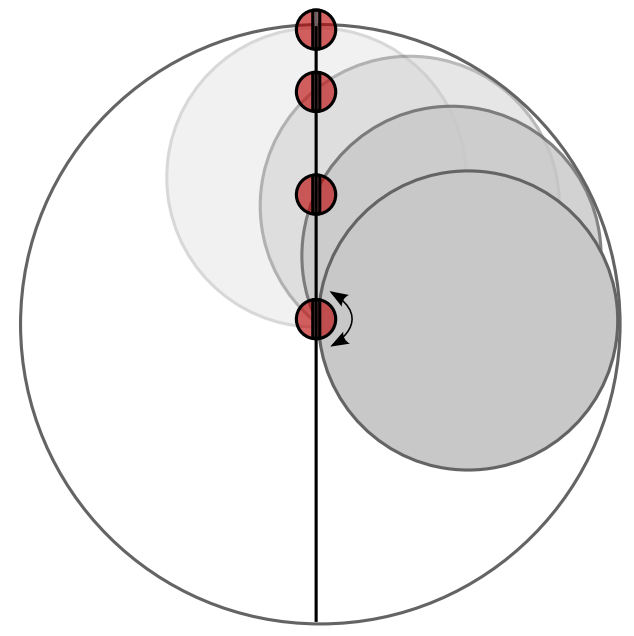

(a)

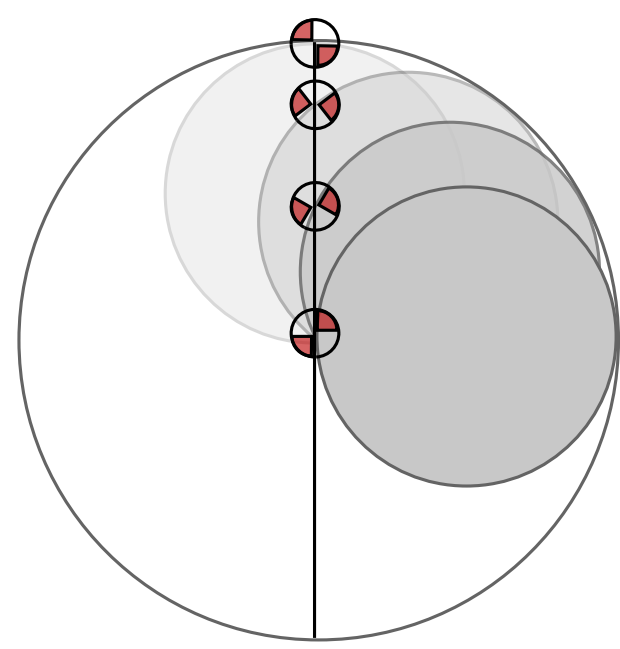

(b)

Figure 4.20: Alternative realizations of the VSM concept. One (a) uses a pin with a groove, the other (b) uses a fixed cylindrical pin with two quarter cylinders cut away (white parts). 


\section{CHAPTER 5}

\section{Single-Motor Design: The SM-VSA}

This chapter presents a proof of concept of a variable stiffness actuator (VSA) that uses only one (high power) input motor. In general, VSAs use two (high power) motors to be able to control both the output position and the output stiffness, possibly resulting in a heavy, and bulky system. In the system presented in this chapter, two small and light-weight clutches are used to lock either one of the degrees of freedom, allowing the other to be controlled by the input motor. These clutches are realized by friction belts that can engage to a surrounding cylinder. The clutches are operated by solenoids, and small bistable mechanisms ensure that no electrical energy is lost in keeping a degree of freedom locked or unlocked. An experiment with a prototype of the system is performed which validates the proof of concept of this Single MotorVSA.

This chapter has been submitted for possible publication as:

[66] S. S. Groothuis, R. Carloni, and S. Stramigioli, "Single motor-variable stiffness actuator using bistable switching mechanisms for independent motion and stiffness control," in Proceedings of the IEEE International Conference on Advanced Intelligent Mechatronics, 2016. Submitted 


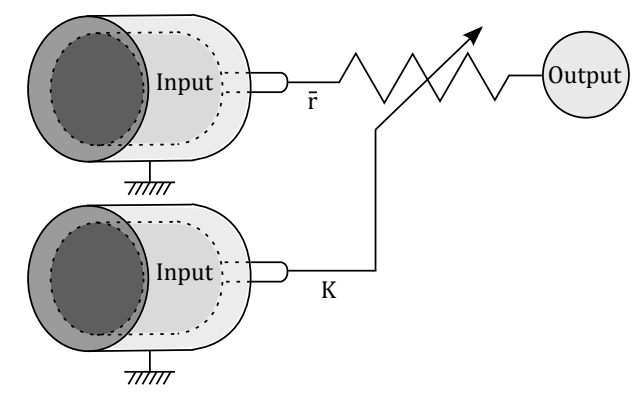

Figure 5.1: A typical VSA uses two distinct motors for independent motion and stiffness control of the VSA.

$\mathrm{T}$ HE need for safe human-robot interaction has led to a paradigm shift of high bandwidth, fast, and stiff robotic systems to slower and (active or passive) compliant systems. Within this paradigm, various safety measures have been developed, among which are VSAs, that are capable of varying their connected load position as well as the compliance that couples the motor to the load. Many different designs have been proposed in the literature, and most of them make use of mechanical springs. These VSAs require two (not necessarily equally) powerful motors to control both the output position and the output stiffness degree of freedom. This means that the device is likely to become bulky and heavy, since powerful motor and gearbox combinations add considerable weight and volume to a system. This is shown for the group of VSAs with variable transmission or variable physical spring properties in Figure 5.1, where two input motors are necessary to control the two degrees of freedom of the variable stiffness mechanism: one for the equilibrium output position and the other for the stiffness. Being able to control both the output position and output stiffness of the system with only one motor reduces the overall weight and volume, which can be achieved by switching between degrees of freedom using, for instance, clutching mechanisms.

Clutching mechanisms in compliant actuators have been proposed earlier. In [67], a clutching mechanism was used in parallel to a driving motor to create both a series elastic actuator (SEA), with a disengaged clutch, and a passive compliant joint, with an engaged clutch. Similarly, in [68] and [69], a clutch mechanism placed in parallel to the elastic element was used to lock a joint in a rigid, high bandwidth operational mode, while disengaging, or slipping, the clutch to ensure compliant interaction. In [19], a series clutch actuator was proposed, which uses electronically adjustable friction clutch discs to limit the applied force at the end effector to a prescribed safe level. Exceeding that level causes the clutch to slip. In [70], these clutches where used to provide friction to brake an artificial muscle-driven joint. In other previous work [71, 72], One-To-Many actuators have been presented, in which one electric motor can charge multiple elastic element modules, that can then release the stored potential energy as kinetic energy on multiple outputs. This means that the energy delivered to the output can never exceed the energy that is present in the elastic elements since the input motor is never directly connected to an output, i.e., actuation is passive. This is desirable for stability and safety, but not desirable if more energy needs to be delivered to an output than the limited amount of energy that can be stored in the elastic element.

In [73], a prototype of a VSA was developed that could switch between operational mode (either position change, or stiffness change) using an auxiliary motor to drive a clutching mechanism. The main motor was then able to actuate both degrees of freedom separately. However, switching between operational modes took approximately 20 seconds making that prototype only usable for a very limited number of, mainly slow-moving, applications. 


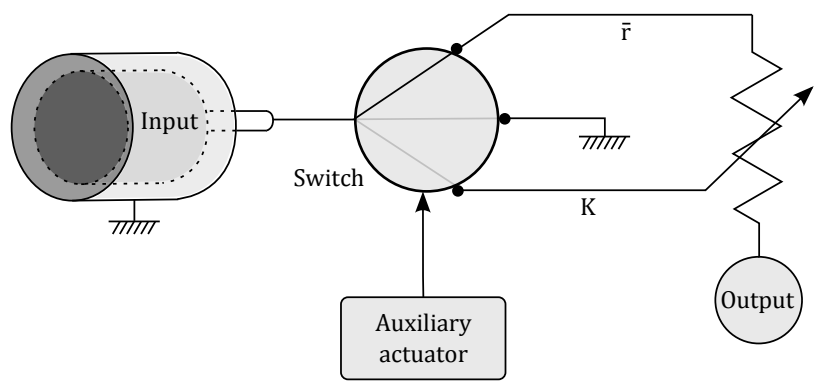

Figure 5.2: Schematic representation of a switching VSA. The input motor is coupled by a switch as position control or stiffness control. The switching is done by an auxiliary actuator.

In this chapter, a novel Single Motor-Variable Stiffness Actuator (SM-VSA) is presented, which uses only one high power input motor, while it is capable of actuating two degrees of freedom. It will be shown that switching between the operational modes is near instantaneous, because of the usage of solenoid-driven bistable mechanisms. In the novel SM-VSA, the input motor is directly connected to one of the degrees of freedom, so the limitation of the finite amount of energy that can be supplied to an output in one actuation phase, is not present.

This chapter is organized as follows: the conceptual analysis of the novel Single MotorVSA is presented in Section 5.1. Then, the design and the manufactured prototype of the SMVSA are treated in Section 5.2. An experiment with this prototype is done and is reported in Section 5.3. The results are discussed in Section 5.4 and the paper concludes with Section 5.5.

\subsection{Conceptual Analysis}

In this section, the conceptual analysis of the SM-VSA is presented, treating its working principle.

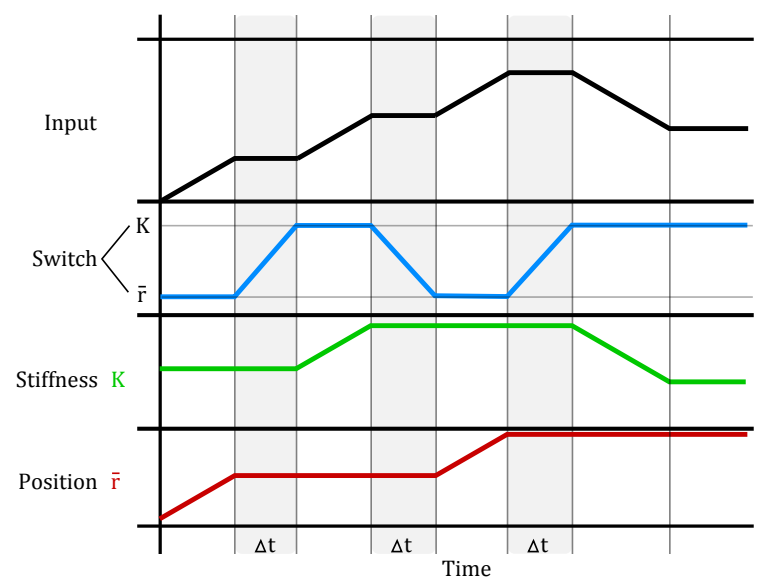

Figure 5.3: An arbitrary motor input profile and an arbitrary switching profile, together with the resulting stiffness and position changes. The switch takes some time $\Delta t$ to switch between the two operational modes, during which the motor is stopped.

Figure 5.2 shows a schematic representation of the SM-VSA. It consists of one input motor 
that is coupled to a switching mechanism. This switching mechanism determines whether the input motor is coupled to a mechanism that controls position, or to a mechanism that controls stiffness. This switching is done by an auxiliary actuator which is never directly coupled to the output and its attached load. The motor can also be coupled to the fixed world, to allow a transition phase.

The procedure of switching between position and stiffness control is shown in Figure 5.3, in which an arbitrary motor input profile and an arbitrary switching profile, together with the resulting stiffness and position are shown. At first, the switch couples the input motor to the position change (the equilibrium output position $\bar{r}$ ), resulting in an increasing equilibrium position. Then, the input motor stops and the switch takes a certain amount of time $\Delta t$ to change from position mode to stiffness mode, after which the motor can be enabled again, resulting in a stiffness change. The second and third $\Delta t$ regions show a similar mode switching procedure.

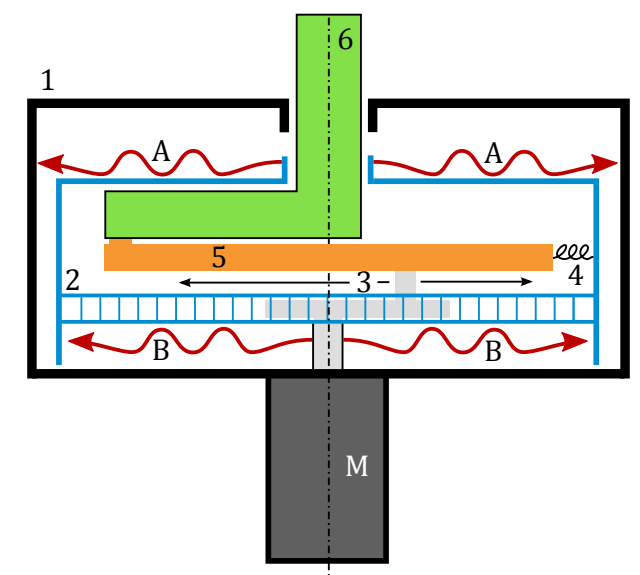

Figure 5.4: A schematic cross section of the novel SM-VSA, which is based on the mVSA-UT [51]. It consists of a frame (1), a rotor (2), a planet carrier and gear with pivot (3) - which forms, together with a ring gear on the rotor (2), the gear mechanism to change the stiffness -, a spring (4), a lever (5) and an output (6). Depending on the state of clutches (A and B), that can couple rotor (2) to frame (1) and planet carrier (3) to rotor (2), respectively, the motor (M) can either actuate the equilibrium output position, or the output stiffness.

The SM-VSA is based on the integrated and miniaturized mVSA-UT [51], which in turn is based on the vsaUT-II [39]. The variable stiffness on the output is realized by using a variable transmission ratio between the internal springs and the output. This transmission ratio is varied by moving a pivot along a lever arm using a hypocycloid gearing mechanism. Figure 5.4 shows a cross section of the SM-VSA. There is one central motor (M) and the frame (1) is attached to its housing. The rotor (2) is the degree of freedom that sets the equilibrium position of the actuator output. Inside this rotor, the gear mechanism is found which consists of a ring gear (connected to the rotor) and a planet carrier with planet gear and pivot (3), which rotate in the ring gear, and thereby move the pivot in a straight line along a lever (5), which rotates around the pivot. Springs (4) are attached to this lever and the output (6) is connected on the other side of the lever.

The novelty in this design lies in the clutches A and B. Clutch A can connect (and lock) the rotor (2) to the frame (1), while Clutch B can connect (and lock) the planet carrier (3) to the rotor (2). The degree of freedom that can be actuated by the motor (M), depends on which clutch is engaged. Table 5.1 reports the various configurations and resulting situations of the SM-VSA. Note that since there are two clutches, the operational procedure is slightly 
different from the one that is graphically depicted in Figure 5.3.

Table 5.1: Various configurations of the novel Single Motor-VSA in Figure 5.4, as a result of an engaged or disengaged switching mechanism state.

\begin{tabular}{cll}
\hline A engaged & A disengaged \\
\hline B engaged & $\begin{array}{l}\text { Fully locked configuration. The } \\
\text { motor cannot move, the output } \\
\text { can deflect passively. }\end{array}$ & $\begin{array}{l}\text { The motor moves the equilib- } \\
\text { rium position, while the stiffness } \\
\text { change is locked (constant stiff- } \\
\text { ness). The output is actuated and } \\
\text { can deflect passively. }\end{array}$ \\
B disengaged & $\begin{array}{l}\text { The motor moves the planet car- } \\
\text { rier, thereby changing the stiff- } \\
\text { ness. The equilibrium position is anderdefined situation. } \\
\text { fixed to the frame and the output } \\
\text { can deflect passively. }\end{array}$ & $\begin{array}{l}\text { The motor moves the planet car- } \\
\text { rier, but the rotor (2) is also free } \\
\text { to move. The output can deflect } \\
\text { passively, but may also backdrive } \\
\text { the rotor (2). This situation is not } \\
\text { desirable and should be avoided. }\end{array}$ \\
&
\end{tabular}

\subsection{The Novel Single Motor-VSA}

In this section, the design of the novel Single Motor-VSA is treated.

\subsubsection{Clutch analysis}

Clutching mechanisms (A and B in Figure 5.4) are needed to be able to switch between actuating one or the other degree of freedom, namely the output position and the output stiffness. In [73], one clutching mechanism was used, consisting of pinions that are raised or lowered by a motor into holes to lock either one of the degrees of freedom. Energy is only required during the switching and a stable situation, in which no energy needs to be supplied, occurs when the switching is complete.

There are two clutching mechanisms in the SM-VSA to be able to switch between the motion or stiffness degree of freedom. They make use of friction belts that expand in diameter to engage to a cylinder directly surrounding it. Friction belts are inexpensive and simple, can be small and light-weight, and can be manufactured easily allowing rapid prototyping techniques [74]. A friction belt inside a surrounding cylinder is shown in Figure 5.5a. This friction belt should be a slightly flexible open circular shape of which the diameter can be enlarged by applying a force at both open ends $\left(T_{1}\right.$ and $\left.T_{2}\right)$. This causes the friction belt to engage uniformly in a surrounding cylinder at angles $\alpha_{1}$ and $\alpha_{2}$, thereby causing friction which blocks the rotation of the friction belt in the cylinder. Note that the friction belt should not buckle, since no tension can be built up in the belt and it does not engage to the cylinder uniformly.

To analyse the forces in the friction belt given in Figure 5.5b, and in particular the holding force before slipping occurs, the force equilibria in parallel (tangential) and perpendicular directions to the friction belt of a small, static section $d l$ of the belt are found, which are integrated over the complete belt $[74,75]$. 


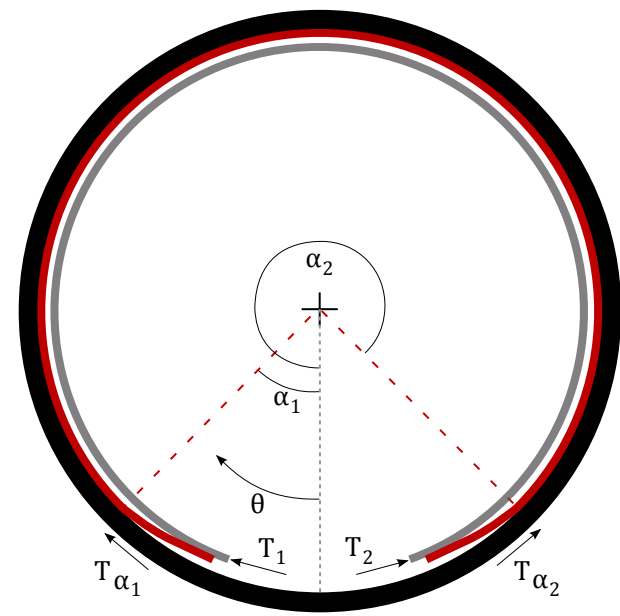

(a) The friction belt inside a surrounding cylinder.

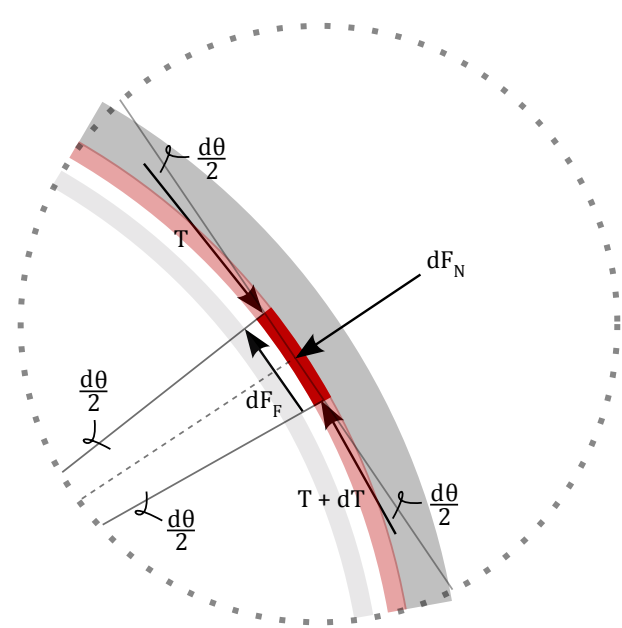

(b) An infinitesimally small section $d l$ of the friction belt, with associated forces.

Figure 5.5: Schematic views of the friction belt inside a cylinder. The symbols represent belt tension $T$, angles of engagement $\alpha_{1}$ and $\alpha_{2}$, circular coordinate $\theta$, friction force $F_{F}$, and normal force $F_{N}$. The tension in the part of the belt that is not engaged with the surrounding cylinder is constant and equal to $T_{1 \mid 2}$. At angle $\alpha_{i}$, the tension equals $T_{\alpha_{i}}=T_{i}$.

For the tangential forces on the belt, it holds that:

$$
\begin{gathered}
\sum F_{\|}=0 \\
-d F_{F}-(T+d T) \cos \left(\frac{d \theta}{2}\right)+T \cos \left(\frac{d \theta}{2}\right)=0 .
\end{gathered}
$$

Here, $F_{F}$ represents the friction force, $T$ is the belt tension and $\theta$ is the circular coordinate. Since the angle $d \theta<<1$, it can be approximated by:

$$
\begin{array}{r}
-d F_{F}-(T+d T)+T \approx 0 \\
-d T=d F_{F}=\mu d F_{N},
\end{array}
$$

where the parameter $\mu$ is the static friction coefficient between the belt and the cylinder, and $F_{N}$ is the normal force to the belt, thus assuming a simple linear static Coulomb friction model.

A similar relation for the perpendicular forces can be found:

$$
\begin{gathered}
\sum F_{\perp}=0 \\
-d F_{N}+(T+d T) \sin \left(\frac{d \theta}{2}\right)+T \sin \left(\frac{d \theta}{2}\right)=0 .
\end{gathered}
$$

Again, approximating the small angle $d \theta<<1$, results in:

$$
\begin{gathered}
-d F_{N}+(T+d T)\left(\frac{d \theta}{2}\right)+T\left(\frac{d \theta}{2}\right)=0 \\
-d F_{N}+T d \theta-d T \frac{d \theta}{2}=0 \\
-d F_{N}+T d \theta=0,
\end{gathered}
$$


where it is used that $d T \frac{d \theta}{2}<<T d \theta$. Then, using (5.1) and (5.2), the following holds:

$$
\begin{aligned}
d F_{N} & =T d \theta \\
\mu d F_{N} & =-d T,
\end{aligned}
$$

and thus:

$$
\begin{gathered}
\mu T d \theta=-d T \\
\frac{d T}{T}=-\mu d \theta
\end{gathered}
$$

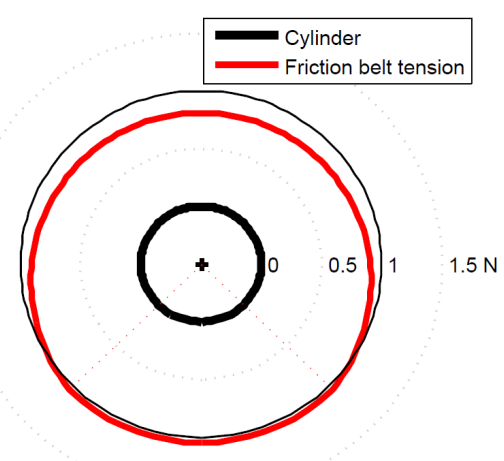

Figure 5.6: The tension force in the friction belt for arbitrary parameters. Two dotted straight lines radially outwards indicate the engagement angle of the belt to the cylinder (cf. Figure 5.5a). The belt tension is symmetric if $T_{1}=T_{2}$. Radially outwards means a higher tension force.

Integrating this over the complete belt as a function of the coordinate $\theta$ :

$$
\begin{aligned}
\int_{T_{\alpha_{1}}}^{T_{\theta}} \frac{1}{T} d T & =-\mu \int_{\alpha_{1}}^{\theta} d \theta \\
\left.\ln (T)\right|_{T_{\alpha_{1}}} ^{T_{\theta}} & =\left.\mu \theta\right|_{\alpha_{1}} ^{\theta} \\
\ln \left(T_{\theta}\right)-\ln \left(T_{\alpha_{1}}\right) & =-\mu\left(\theta-\alpha_{1}\right) \\
\ln \left(\frac{T_{\theta}}{T_{\alpha_{1}}}\right) & =-\mu\left(\theta-\alpha_{1}\right) \\
\frac{T_{\theta}}{T_{\alpha_{1}}} & =e^{-\mu\left(\theta-\alpha_{1}\right)} \\
T_{\theta} & =T_{\alpha_{1}} e^{-\mu\left(\theta-\alpha_{1}\right)}=T(\theta),
\end{aligned}
$$

where $\theta \geq \alpha_{1}$. This is for a single applied tensile force $T_{1}$. By symmetry and superposition, the belt tension as a result of an applied force $T_{2}$ is:

$$
T(\theta)=T_{\alpha_{2}} e^{\mu\left(\theta-\alpha_{2}\right)},
$$

where $\theta \leq \alpha_{2}$. Hence, the tension in the belt as a function of coordinate $\theta$ can be parameter- 
ized by:

$$
\begin{aligned}
T(\theta)=T_{\alpha_{1}}+T_{\alpha_{2}} e^{\mu\left(\theta-\alpha_{2}\right)} & \text { for } 0 \leq \theta<\alpha_{1} \\
T(\theta)=T_{\alpha_{1}} e^{-\mu\left(\theta-\alpha_{1}\right)}+T_{\alpha_{2}} e^{\mu\left(\theta-\alpha_{2}\right)} & \text { for } \alpha_{1} \leq \theta \leq \alpha_{2} \\
T(\theta)=T_{\alpha_{2}}+T_{\alpha_{1}} e^{-\mu\left(\theta-\alpha_{1}\right)} & \text { for } \alpha_{2}<\theta \leq 2 \pi .
\end{aligned}
$$

With this, the total (holding) friction force of the belt on the surrounding cylinder can be found. From (5.3), the relation between friction force and the tension on an infinitesimally small belt section is known:

$$
\begin{gathered}
d F_{F}=\mu T d \theta \\
\int_{F_{F_{\alpha_{1}}}}^{F_{F_{\alpha_{2}}}} d F_{F}=\mu \int_{\alpha_{1}}^{\alpha_{2}} T d \theta .
\end{gathered}
$$

Then, using (5.4) and integrating over the complete belt in contact with the cylinder, the total (holding) friction force becomes:

$$
F_{F}=\left(T_{\alpha_{1}}+T_{\alpha_{2}}\right)\left(1-e^{-\mu\left(\alpha_{2}-\alpha_{1}\right)}\right) .
$$

In Figure 5.6, the tension force is graphically depicted in a polar plot for arbitrary values of the friction parameter $\mu$, angles $\alpha_{1}$ and $\alpha_{2}$, and the cylinder diameter. The applied tension force on the belt is $T_{1}=T_{2}=1 \mathrm{~N}$. There are two dotted straight lines radially outwards from the center, indicating the angles of engagement of the belt with the cylinder. The angle in this plot corresponds to the physical angle of a cylinder (cf. Figure 5.5a). It can be seen that the tension force is constant for the section of the belt that is not in contact with the cylinder (bottom part), and that it is indeed symmetric if $T_{1}=T_{2}$.

\subsubsection{Bistable switching mechanism}

\section{Auxiliary actuators}

The auxiliary actuators should be translational actuators to generate the forces $T_{1}$ and $T_{2}$ that are necessary to enlarge the friction belt, as shown in Figure 5.5. The actuators should preferably be electric, so that the control system and the prototype itself are simple and straightforward without needing much additional equipment. Therefore, pneumatic actuation, for example, was not considered. Piezo stacks or piezo benders deform when an electric field is applied (or vice versa). This deformation can be directly used to engage or disengage the clutch. Although these piezo elements can deliver much force when a high voltage is applied, their stroke or deformation is very limited, i.e., often less than a millimeter. Therefore, solenoids are used as auxiliary actuators, to generate the force that is necessary to enlarge the friction belt. They can deliver high forces with acceptable strokes and are relatively small, which allows an easy integration. Specifically, the Kuhnke HU 244 linear solenoids were used.

\section{Bistable switches}

The friction belts are the clutches to lock or unlock degrees of freedom, for which a continuous applied force is needed. It is not desirable to use the solenoids to directly generate this force, since this requires electrical energy, while no mechanical work is performed. Therefore, a bistable mechanism was used to keep the friction belt in either the locked or the unlocked 


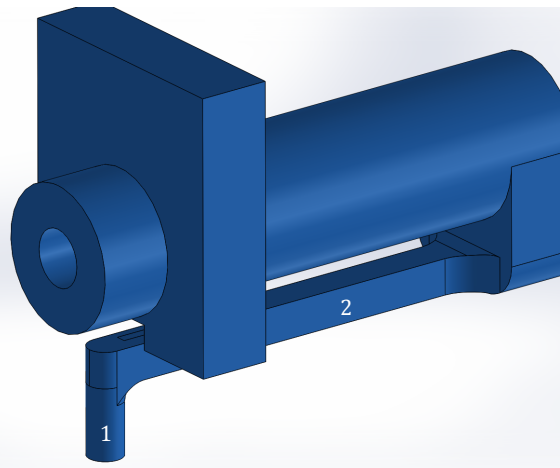

(a) A pin-shaped cam follower (1) attached to a flexure (2).

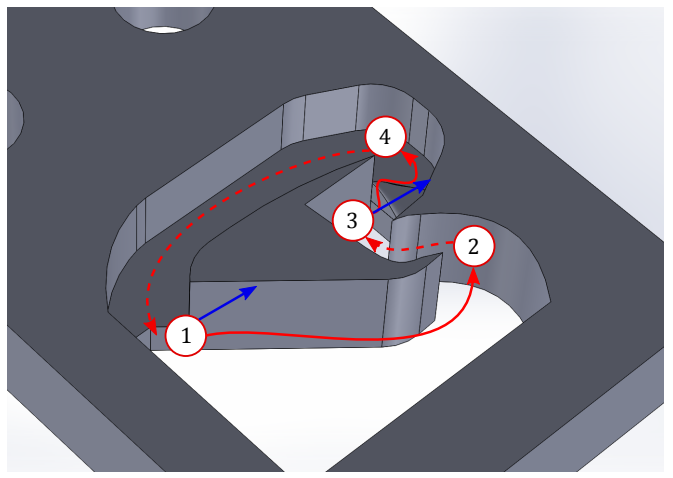

(b) The cam in which the cam follower moves, with stable position (1) and (3), and intermediate position (2) and (4). The red solid line shows actuated movement, while the dashed line indicated relaxation movement.

Figure 5.7: The bistable mechanism consists of a cam profile and a cam follower.

state. The bistable mechanism, shown in Figure 5.7, is inspired by one of the many mechanisms that can be found in a ballpoint pen. It uses a cam follower (Figure 5.7a) and a cam profile with two stable positions (Figure 5.7b).

The pin-shaped cam follower is unloaded at position (1) in Figure 5.7b. When a force is applied (blue vector, representing the solenoid force), the flexure of the cam follower deflects and the pin moves to position (2). When the force is removed, the loaded flexure causes the pin to move to stable position (3). When again a force is applied in the direction of the vector, the pin moves to position (4), and when the force is removed, the enlarged and therefore loaded friction belt (pushing the cam follower in the opposite direction of the force vector shown) causes the pin to move to position (1) again. Figure 5.8 shows the complete assembled bistable mechanism with friction belt. If the solenoid is powered very briefly, the friction belt enlarges and stays enlarged. No electrical energy is consumed to keep the mechanism in this state. When the solenoid is powered again, the friction belt returns to its initial state.

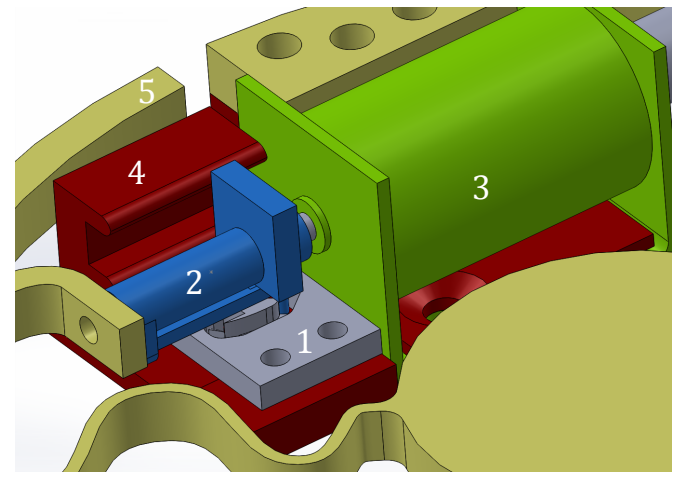

(a) Close-up view of the bistable mechanism.

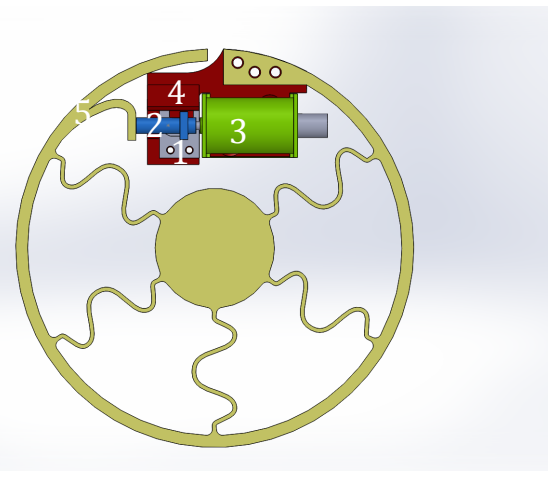

(b) Top view of the bistable mechanism.

Figure 5.8: The assembled friction belt with bistable mechanism and solenoid. The labels refer to the cam (1), the cam follower (2), the solenoid actuator (3), a bracket for assembling (4), and the friction belt (5). 


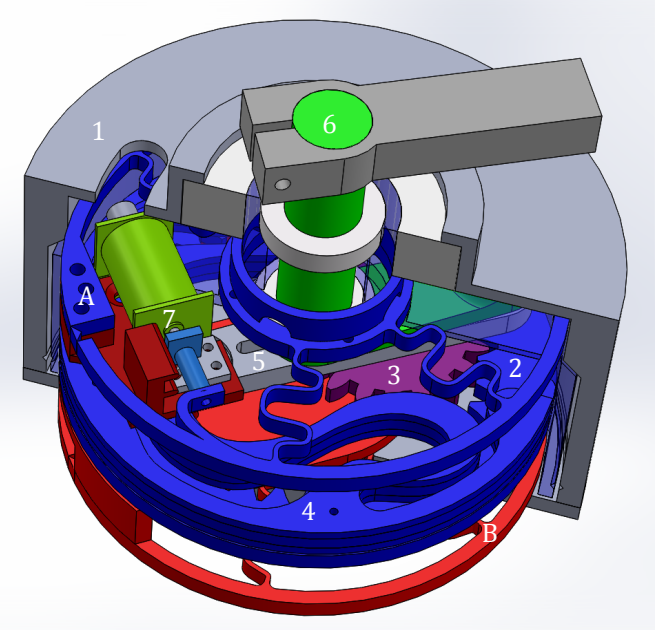

(a) Top view, showing the bistable mechanism (7) and friction belt (A) to lock the equilibrium position to the frame (1).

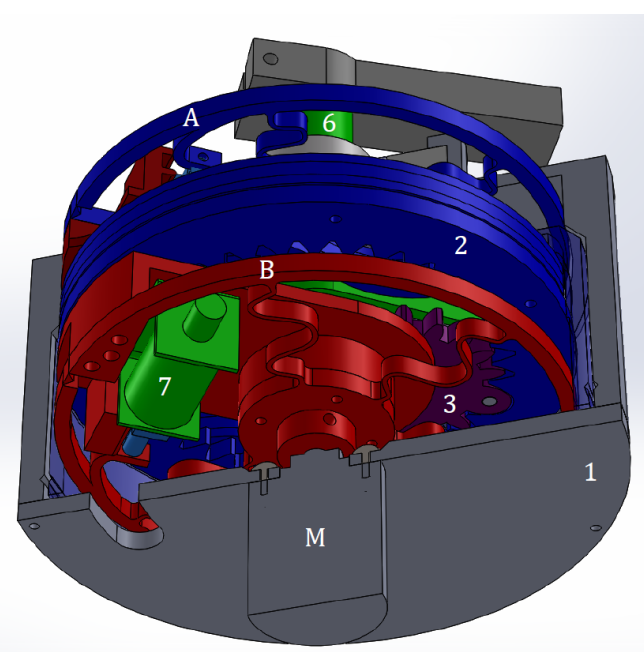

(b) Bottom view, showing the bistable mechanism (7) and friction belt $(B)$ to lock the stiffness change degree of freedom.

Figure 5.9: A CAD drawing of the SM-VSA, where the labels are similar to those in Figure 5.4. The frame, or housing, is indicated with (1), the rotor (2) consists of an enclosing frame (shown transparent) within housing (1) connecting the ring gear, (leaf) spring (4), and friction belt (A), a planet gear with pivot (3) sits on a planet carrier to which the second friction belt is connected (B), the lever arm (5) connects the springs to the output (6), and the friction belts are actuated by the bistable mechanisms (7).

\subsubsection{The SM-VSA design and prototype}

A cross sectional view of the SM-VSA is shown in Figure 5.9, and an exploded view of the SM-VSA is shown in Figure 5.10. The numeric labels, identical to the ones in Figure 5.4, refer to the frame (1), the rotor with attached ring gear (2), the planet gear on top of the planet carrier (3), the (leaf) spring (4), the lever arm with groove for the moving pivot (5), the output with output arm (6), and one of the two bistable mechanisms (7). Both friction belts (A and B) can be distinguished. Friction belt (A) and the corresponding bistable mechanism with solenoid rotate together with rotor (2) inside frame (1), when the clutch is not engaged to frame (1). Likewise, friction belt (B) and the corresponding bistable mechanism rotates together with the planet carrier (3) inside rotor (2), when the clutch is not engaged to rotor (2).

A prototype of this system was manufactured, using rapid prototype techniques, i.e., laser cutting and 3D-printing. The finished prototype is shown in Figure 5.11. The frame, indicated with (1), was 3D-printed and has a diameter of $120 \mathrm{~mm}$ and a height of $64 \mathrm{~mm}$. Including the output (6), which was also 3D-printed, the height is $99 \mathrm{~mm}$. Furthermore, the rotor, the lever, the planet carrier, the cam and cam follower were 3D-printed as well. The friction belts, the springs, and all gears were laser cut from plastic. Friction tape was put on the friction belts to increase the friction coefficient. The solenoids are powered using a custom charge and discharge circuit, in which a $C=30 \mu \mathrm{F}$ capacitor is fully charged in $200 \mathrm{~ms}$, and discharges over the particular solenoids used in $20 \mathrm{~ms}$. When it discharges, it is ensures that the power supply is disconnected from the capacitor, so that never more charge than what is in the capacitor will be released through the solenoid to prevent overheating. A supply voltage of $U=130 \mathrm{~V}$ ensures a proper operation of the bistable mechanisms by the solenoids. This means that the energy to switch between operational modes, which requires switching 


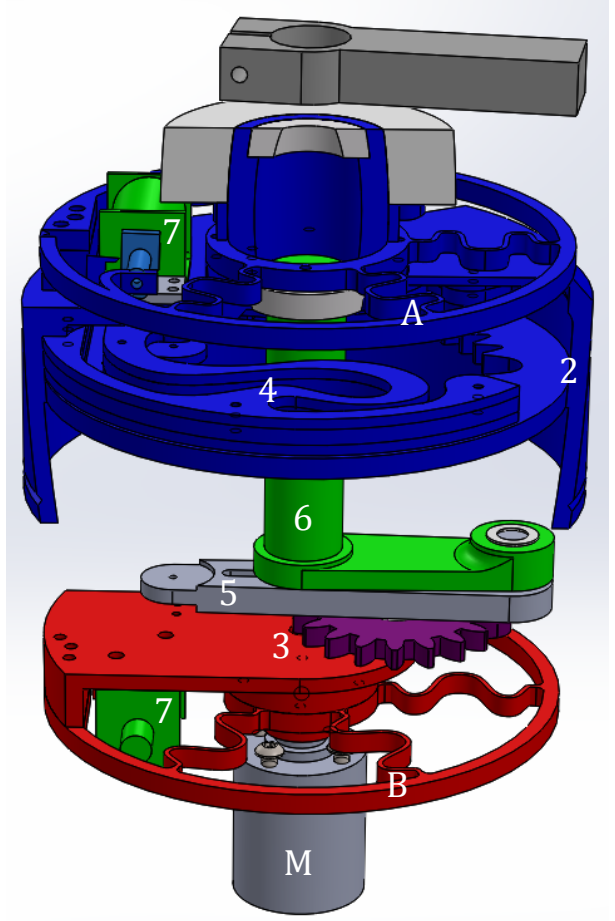

Figure 5.10: Exploded, and partially cross-sectional, view of the SM-VSA, with the same labels as in Figure 5.9. The enclosing frame of the rotor (2), and the planet carrier (3) to which the planet gear of the gear mechanism is connected are now shown more explicitly.

operation in both mechanisms, is $2 \cdot E_{\text {switch }}=2 \cdot \frac{1}{2} C U^{2} \approx 0.5 \mathrm{~J}$. In principle, it would take $40 \mathrm{~ms}$ to complete the switching.

\subsection{Experimental Validation}

An experiment was performed with the prototype of the system. An Arduino was used as software platform. The SM-VSA can be controlled with two buttons, to power the two solenoids, and a potentiometer to set the velocity of the input motor. The motor has an incremental optical encoder for measuring its position and velocity and an absolute magnetic encoder was used to measure the output angle. The motor is controlled in velocity mode and the Arduino communicates with the motor controller to set and measure the position and velocity. The Arduino sends the measurements of the encoders and the states of the buttons to Matlab Simulink through a serial RS232 connection. In Simulink, the data is acquired and passed on to the work space for further processing.

The experimental result is shown in Figure 5.12. It starts with a locked stiffness degree of freedom (so the stiffness is constant, at the stiffest setting), while the motion degree of freedom is unlocked. The motor is then turned, which moves the output as expected. Then the motion degree of freedom is also locked, to ensure that the system remains in the current configuration and does not become underdefined, after which the stiffness degree of freedom is unlocked. In this experiment the switching was done slowly (the grey shaded area is approximately 2 seconds wide), which was done to clearly indicate the switching sequence. 
In principle, this can be done in $40 \mathrm{~ms}$. The motor is then turned again during interaction with the output (manually tapping), which results in an oscillation-like behavior with growing amplitude, because of the decreasing stiffness (increasing compliance). When the most compliant setting is reached, the stiffness degree of freedom is locked again, the motion degree of freedom is unlocked and the motor then rotates the output again. Some interaction occurs, showing that the output compliance is properly kept.

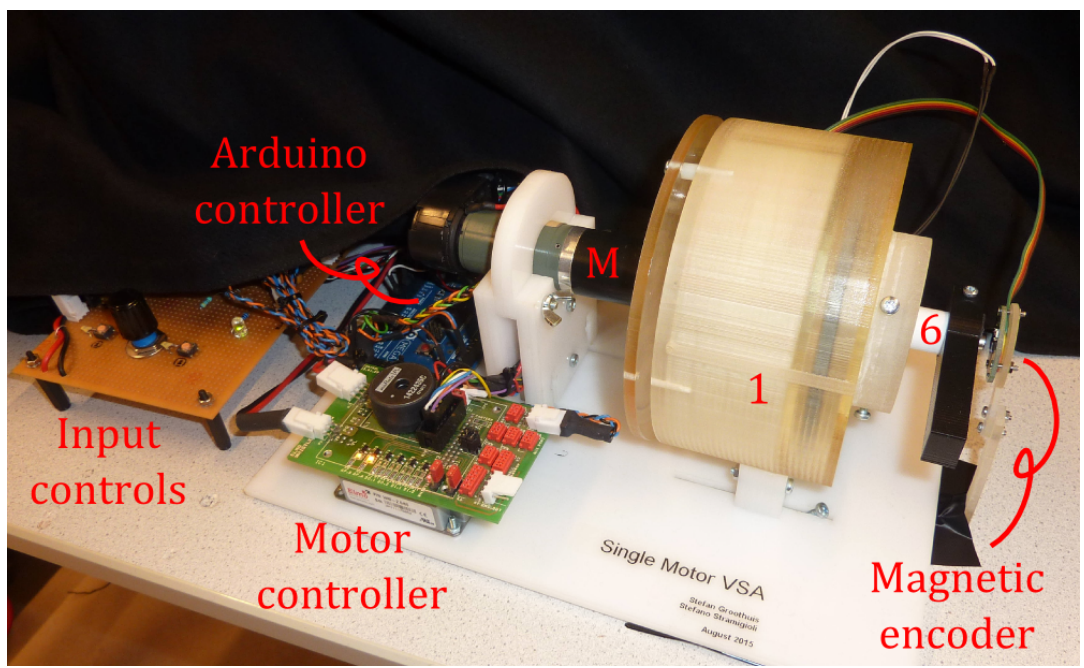

Figure 5.11: Prototype of the novel Single Motor-VSA.

\subsection{Discussion}

The experiment validates the proof of concept: it is possible to control both degrees of freedom of a VSA with only one input motor. Obviously, there is always a need for more than one actuator to be able to do this, but the two auxiliary actuators that are needed in this prototype to switch between degrees of freedom, are small, light-weight, and only briefly powered to lock or unlock a degree of freedom almost instantaneously. This means that one high power, and relatively large and heavy motor is sufficient and no additional large, heavy, and high power motors are not necessary.

The prototype has been designed to have the ability to manufacture with rapid prototyping facilities. This means that the focus was put on an integrated cylindrical design that features two mechanical interfaces, i.e., the input motor and the output shaft, and not so much on having the most compact design. An issue with the current design is that continuous and multiple rotations are not possible because of wires going to the solenoids. This is easily solved, however, by using slip rings that transmit the electrical power to the rotating elements.

Traditional VSAs are capable of simultaneous actuation of position and stiffness, while this prototype is not. Here, only one of the two degrees of freedom can be actuated, which would suit applications in which the stiffness may be constant during motion, but where it is important that one can switch to actuating the output stiffness immediately. However, it may be possible, when a mechanism is used that can split input power in a desired way (e.g. [76]), to output to the two degrees of freedom (e.g. $70 \%$ motion, $30 \%$ stiffness).

Although the proof of concept has been validated, further work needs to be done to char- 
acterize the system, and to examine the scalability of this system and prototype to certain (higher) output loads.

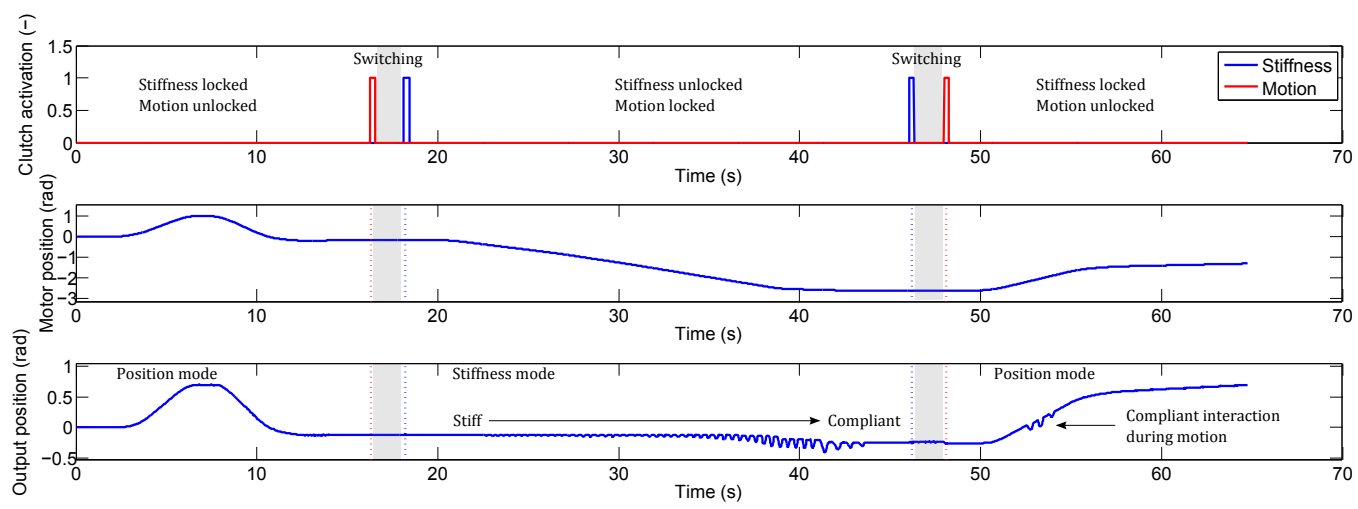

Figure 5.12: Experimental validation of the concept. Clutch activation refers to the state of the motion and stiffness bistable mechanisms: a high signal means that the solenoid is powered, causing the locking or unlocking of the switch. First the motion degree of freedom is unlocked and actuated, after which at 16 seconds, the operational mode is switched to stiffness actuation. In this experiment the switching was done slowly (the grey shaded area is approximately 2 seconds wide), which was done to clearly indicate the switching sequence. The stiffness actuation mode starts in the stiffest setting and gradually goes to the most compliant setting, while the output is continuously disturbed (by manually tapping). At 46 seconds, the operational mode is switched again to actuate the motion, during which interaction with the compliant output occurs.

\subsection{Conclusion}

This paper has presented a novel variable stiffness actuator using only one (high power) input motor. The Single Motor-VSA (SM-VSA) is able to actuate position and stiffness by locking and unlocking a degree of freedom in the VSA (either position or stiffness). This is done by bistable friction belt mechanisms, in which the diameter of a circular shaped flexure (the friction belt) is enlarged in diameter, causing it to engage to a surrounding cylinder, which generates friction and, thereby, locks a degree of freedom. The bistable mechanism ensures that the solenoids, that are used for the enlargement of the friction belts, can be very briefly powered, such that no electric energy is wasted in keeping a certain degree of freedom locked or unlocked. A prototype of the system was presented and an experiment was performed, which validates the novel concept. 
PART II

Compliant Robotic Systems on Graphs 
CHAPTER 6

\section{Variable Stiffness Actuators on Graphs}

In this chapter, a modular method of modeling compliant robotic systems using graph theory is treated. Graph theoretic analyses ensure a structured way of describing a system and allow a straightforward extension to more complex systems. The graph models of a series elastic actuator, a variable stiffness actuator and a multiple degrees of freedom compliant system are derived. These systems are controlled using an optimal control law that is able to find the optimal stiffness setting and distribution to accomplish a certain task. A case study shows a multiple degrees of freedom compliant system which is required to resonate at the output and to accomplish a back and forth motion. It is shown that a constant optimal stiffness is found in the resonance simulation, and a varying optimal stiffness in case of the back and forth task. This indicates that this methodology can assist in finding an optimal stiffness distribution of complex robotic systems for a given task.

This chapter has been previously published as:

[77] S. Groothuis, S. Stramigioli, and R. Carloni, "Compliant robotic systems on graphs," in Proceedings of the IEEE/RSJ International Conference on Intelligent Robots and Systems, 2014.

(c) 2014 IEEE 


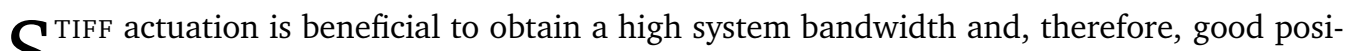
$\mathcal{S}$ tion accuracy and high speeds. However, and very importantly for physical human-robot interaction, stiff actuation has a significant drawback, namely: it is not intrinsically safe, since active impedance control cannot be robustly implemented because of the finite system bandwidth. Therefore, series elastic actuation (SEA) has been designed, featuring a physical elastic element, to robustly measure elastic deflection and, thereby, controlling the forces that are applied to the environment. Besides SEA, variable stiffness actuation (VSA) [64] has the additional property to adjust the internal elastic element such that the output stiffness, which is felt at the actuator output, can be adjusted. These actuators provide a better safety and they are, therefore, more suitable for human-robot interaction, as was shown in Chapter 2 [4]. Over the past years, the VSA technology has had a considerable increase in research effort, resulting in lots of different actuator designs and control strategies. The benefit of variable stiffness may be exploited using for instance optimal control to minimize the control power and optimally control with respect to an objective [78, 79, 80].

Graph theoretic models are very ubiquitous in that they can model very different systems and phenomena, e.g., transportation systems, chemical networks, social models and mechanical engineering applications like mass-spring-damper systems [81] and multibody systems with or without external forces [82, 83]. In [84], static truss structures are studied by associating the physical entities (masses, springs, dampers and actuators) to the edges of a graph, which then connect to nodes associated to the vertices. Also, dynamical systems have been modeled in the port-Hamiltonian framework using graph theory [85], where masses are associated to vertices, that are interconnected by springs, dampers and actuators associated to the edges. Graph theory has been used to model multibody engineering systems with force loads, where these force loads are idealized representations of actuators present in the system. However, the actuators that are considered are stiff, i.e., there is no physical elastic element incorporated in the actuator.

This chapter proposes a modular way of modeling compliant actuators, i.e., SEAs and VSAs, using graph theory and shows the possibility to optimize the stiffness distribution in complex compliant systems. Graph theoretic analyses ensure a structured way of describing a system and performing calculations. This holds even in case of very large dimensional systems, since Kirchhoff's balance laws are valid for all physical systems. The graph theoretic modeling of compliant actuators, as presented in this chapter, allows a modular approach to the synthesis of large dimensional systems using multiple compliant actuators. The increase in system complexity is minimal, due to the straightforward laws of graph theory. Moreover, it will be shown by a case study that this method can easily assist in finding an optimal stiffness distribution of complex robotic systems of increasing complexity for a given task when utilizing an optimal control scheme. It shows whether variable stiffness actuation may be superfluous in some joints for achieving a task, such that proper design decisions can be made.

First, in Section 6.1, the basics of graph theory are elaborated and Section 6.2 presents the graph theoretic models of compliant actuators. In Section 6.3, a multiple degrees of freedom model is controlled to achieve a certain task while optimizing the actuators' stiffness. The method and its results are discussed and concluded in Section 6.4 and Section 6.5.

\subsection{Graph Theory}

A directed graph $\mathfrak{G}$ is defined by a set of $n$ vertices $\mathbb{V}_{\mathfrak{G}}=\left\{\mathrm{v}_{1}, \mathrm{~V}_{2}, \ldots, \mathrm{V}_{n}\right\}$ and a set of $m$ directed edges $\mathbb{E}_{\mathfrak{G}}=\left\{\mathrm{E}_{1}, \mathrm{E}_{2}, \ldots, \mathrm{E}_{m}\right\} \subseteq \mathbb{V}_{\mathfrak{G}} \times \mathbb{V}_{\mathfrak{G}}$, i.e., a set of ordered pairs of vertices defined by the Cartesian product $\times$, which defines the connections between the vertices. An undirected graph consists of edges that are unordered pairs of vertices. 


\section{Graph matrices}

A graph is completely defined by its adjacency matrix $A_{\mathfrak{G}} \in \mathbb{R}^{n \times n}$. For a weighted and directed graph, i.e., the edges are weighted by weights $\mathrm{w}_{1}, \mathrm{w}_{2}, \ldots, \mathrm{w}_{m}$, element $(i, j)$ of the adjacency matrix is defined by:

$$
\left(A_{\mathfrak{G}}\right)_{i j}:=\left\{\begin{array}{cl}
\mathrm{w}_{\bullet} & \text { if there is a directed edge from } \mathrm{v}_{j} \text { to } \mathrm{v}_{i}, \\
0 & \text { otherwise. }
\end{array}\right.
$$

The adjacency matrix of an undirected graph is symmetric. Moreover, if no self-loops, given by $\left(\mathrm{E}_{\bullet}=\left\{\mathrm{V}_{i}, \mathrm{~V}_{i}\right\}\right.$ for $\left.i \in\{1 \ldots n\}\right)$, are allowed, the elements on the diagonal are zero. If the graph is not weighted, all weights are $\mathrm{w}_{\bullet}=1$.

The incidence matrix $B_{\mathfrak{G}} \in \mathbb{R}^{n \times m}$ defines whether edges are incident to vertices. For a directed graph, element $(j, l)$ is given by:

$$
\left(B_{\mathfrak{G}}\right)_{j l}=\left\{\begin{array}{cl}
1 & \text { if edge } \mathrm{E}_{l} \text { enters vertex } \mathrm{v}_{j} \\
-1 & \text { if edge } \mathrm{E}_{l} \text { leaves vertex } \mathrm{v}_{j} \\
0 & \text { otherwise }
\end{array}\right.
$$

For an undirected graph, an arbitrary direction can be assigned to find the incidence matrix. Note that the edge weights do not appear in the incidence matrix, since it only captures the direction of the edges.

The Laplacian matrix $L_{\mathfrak{G}} \in \mathbb{R}^{n \times n}$ of a directed and weighted graph $\mathfrak{G}$ is defined by:

$$
L_{\mathfrak{G}}:=D_{\text {in }}-A_{\mathfrak{G}},
$$

where $D_{\text {in }} \in \mathbb{R}^{n \times n}$ is the diagonal in-degree matrix capturing the sum of the weights of edges incident to a vertex. Since for a directed graph $A_{\mathfrak{G}}$ is in general not symmetric, the Laplacian $L_{\mathfrak{G}}$ is also not symmetric. When the graph is undirected, $A_{\mathfrak{G}}$ and, therefore, $L_{\mathfrak{G}}$ are symmetric. For an undirected graph, the Laplacian may be calculated by:

$$
L_{\mathfrak{G}}=B_{\mathfrak{G}} W_{\mathfrak{G}} B_{\mathfrak{G}}^{T},
$$

where $W_{\mathfrak{G}}:=\operatorname{diag}\left(w_{1}, w_{2}, \ldots, w_{m}\right) \in \mathbb{R}^{m \times m}$ and $w_{\bullet} \geq 0$. When graph $\mathfrak{G}$ is not weighted, $W_{\mathfrak{G}}=I_{m \times m}$ (since $w_{\bullet}=1$ ), where $I_{m \times m} \in \mathbb{R}^{m \times m}$ represents the identity matrix of dimension $m$. It can be seen that the Laplacian is a positive semidefinite matrix and it is independent of the orientation of the graph, e.g., the arbitrary direction assigned to the edges to obtain the incidence matrix.

The Laplacian matrix can be used in the calculation of consensus dynamics. In consensus dynamics, vertices are agents $\mathrm{v}_{i}$, for $i \in\{1,2, \ldots, n\}$, that communicate their state $x_{i}(t)$ to one another via the edges defined by the graph topology, to come to a consensus on their state. When single integrator agents have access to the relative state information of their neighbors, the dynamics can be written as [86]:

$$
\dot{\bar{x}}(t)=-L_{\mathfrak{G}} \bar{x}(t)
$$

where $\bar{x}(t):=\left[\begin{array}{lll}x_{1}(t) & \ldots & x_{n}(t)\end{array}\right]^{\mathrm{T}}$. In case of double integrator agents, the dynamics can be written as [87]:

$$
\ddot{\bar{x}}(t)=-L_{\bar{x}, \mathfrak{G}} \bar{x}(t)-L_{\dot{\bar{x}}, \mathfrak{G}} \dot{\bar{x}}(t)
$$

where $L_{\bar{x}, \mathfrak{G}}$ is the agent position Laplacian and $L_{\dot{\bar{x}}, \mathfrak{G}}$ is the agent velocity Laplacian, calculated on the graph along which the positions and velocities are communicated, respectively. This 


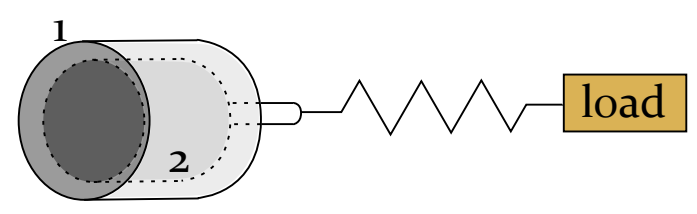

(a) Series elastic actuator.

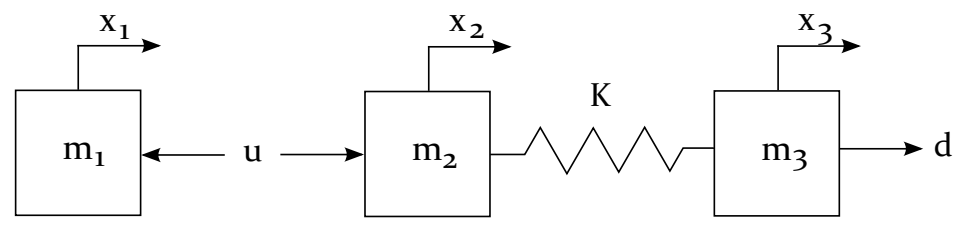

(b) Idealized physical model of a SEA.

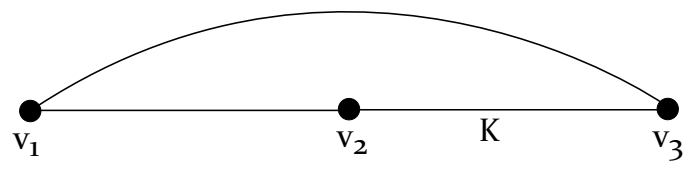

(c) Undirected graph $\mathfrak{G}$ of a SEA.

Figure 6.1: Schematic representation of a SEA (with a stator 1, rotor 2 and a load) (a), together with its idealized physical model (b), and the proposed undirected graph model (c).

form is used in Section 6.2 to model compliant actuators. When (6.1) is expressed in matrix form, one obtains:

$$
\left[\begin{array}{c}
\dot{\bar{x}}(t) \\
\ddot{\bar{x}}(t)
\end{array}\right]=\left[\begin{array}{cc}
0_{n \times n} & I_{n \times n} \\
-L_{\bar{x}, \mathfrak{G}} & -L_{\dot{\bar{x}}, \mathfrak{G}}
\end{array}\right]\left[\begin{array}{c}
\bar{x}(t) \\
\dot{\bar{x}}(t)
\end{array}\right],
$$

where $0_{n \times n}$ is the matrix of all zeros with dimensions $n \times n$.

\subsection{Compliant Actuators on Graphs}

Using the theory of Section 6.1, two compliant actuator models are presented here, namely the SEA and VSA.

\subsubsection{Series elastic actuator}

A schematic representation of a SEA is shown in Figure 6.1a. The stator of the actuator is indicated with 1 and the rotor with 2 . A force can be applied between the stator and rotor to move the load, that is connected to the elastic element. The corresponding idealized physical model is shown in Figure 6.1b. In this figure, $m_{1}$ represents the stator at position $x_{1}, m_{2}$ represents the rotor that sets the equilibrium load position $x_{2}$, and $m_{3}$ represents the load at the actuator output at position $x_{3}$. The ideal force source between the stator and rotor is indicated by input $u$. Moreover, a disturbance force $d$ can be applied to the load. The proposed corresponding undirected graph $\mathfrak{G}$ of this model is given in Figure 6.1c. Three vertices can be distinguished $(n=3)$, i.e., one for each mass, and the vertices are connected by three edges $(m=3)$ representing the input force $u$, the output stiffness $K$ and the disturbance force $d^{1}$. This graph can be divided into subgraphs $\mathfrak{G}_{u}, \mathfrak{G}_{K}$ and $\mathfrak{G}_{d}$ such that $\mathfrak{G}=\mathfrak{G}_{u} \cup \mathfrak{G}_{K} \cup \mathfrak{G}_{d}$, as shown in Figure 6.2, where an edge direction is assigned. The number of vertices and

\footnotetext{
${ }^{1}$ It is assumed that the disturbance force on $v_{3}$ acts with respect to the stator $v_{1}$. Note that this could be extended to disturbances that do not act with respect to a vertex within the model boundary.
} 


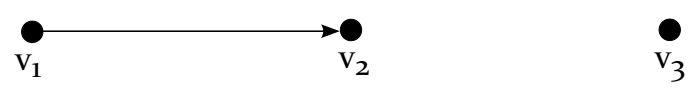

(a) Subgraph $\mathfrak{G}_{u}$ of graph $\mathfrak{G}$ associated to the input force edge.
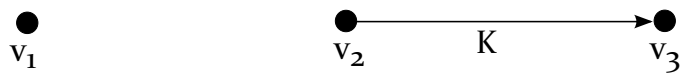

(b) Subgraph $\mathfrak{G}_{K}$ of graph $\mathfrak{G}$ associated to the stiffness edge with weight $K$.

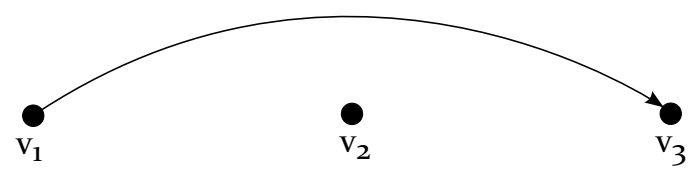

(c) Subgraph $\mathfrak{G}_{d}$ of graph $\mathfrak{G}$ associated to the disturbance edge.

Figure 6.2: Subgraphs of the SEA graph $\mathfrak{G},(a) \mathfrak{G}_{u}$, (b) $\mathfrak{G}_{K}$ and (c) $\mathfrak{G}_{d}$.

edges for these subgraphs are given by $n$ and $m$ with corresponding subscript $u, K$ and $d$, e.g., $m_{K}=1$ for the number of edges in the subgraph $\mathfrak{G}_{K}$. Note that $K$ in the subgraph $\mathfrak{G}_{K}$ is a weight on the edge and that the other edges of graph $\mathfrak{G}$ have no weights associated to them. The incidence matrix $B_{\mathfrak{G}}:=\left[\begin{array}{ccc}B_{\mathfrak{G} u} & B_{\mathfrak{G} K} & B_{\mathfrak{G} d}\end{array}\right]$ is given by the incidence matrices of the subgraphs $^{2}$ :

$$
\begin{aligned}
B_{\mathfrak{G} u} & =\left[\begin{array}{ccc}
-1 & 1 & 0
\end{array}\right]^{\mathrm{T}} \\
B_{\mathfrak{G} K} & =\left[\begin{array}{lll}
0 & -1 & 1
\end{array}\right]^{\mathrm{T}} \\
B_{\mathfrak{G} d} & =\left[\begin{array}{ccc}
-1 & 0 & 1
\end{array}\right]^{\mathrm{T}} \\
B_{\mathfrak{G}} & =\left[\begin{array}{ccc}
-1 & 0 & -1 \\
1 & -1 & 0 \\
0 & 1 & 1
\end{array}\right] .
\end{aligned}
$$

Since a SEA is considered, i.e., an elastic actuator which has ideally no damping, the term $\dot{\bar{x}}(t)$ in (6.1) is neglected. Hence, the dynamics of the SEA are given by:

$$
M \ddot{\bar{x}}(t)=-L_{\mathfrak{G} K}(K) \bar{x}(t)+B_{\mathfrak{G} u} u(t)+B_{\mathfrak{G} d} d(t),
$$

where $\bar{x}(t)$ is the position of the vertices, input force $u(t)$ and disturbance force $d(t)$ are separate inputs in the system and $M \in \mathbb{R}^{n \times n}$ is the diagonal vertex weights matrix representing the vertex inertia. The Laplacian $L_{\mathfrak{G} K}(K)$ is associated to the stiffness subgraph $\mathfrak{G}_{K}$ and is, therefore, a function of the output stiffness $K$ and is calculated by:

$$
L_{\mathfrak{G} K}(K)=B_{\mathfrak{G} K} W_{\mathfrak{G} K} B_{\mathfrak{G} K}{ }^{\mathrm{T}}=\left[\begin{array}{ccc}
0 & 0 & 0 \\
0 & K & -K \\
0 & -K & K
\end{array}\right],
$$

where $W_{\mathfrak{G} K}:=\operatorname{diag}(K) \in \mathbb{R}^{m_{K} \times m_{K}}$ is a diagonal weights matrix on the edges given by $\mathbb{E}_{\mathfrak{G} K}=$ $\left\{\mathrm{E}_{1 K}, \mathrm{E}_{2 K}, \ldots, \mathrm{E}_{m_{K} K}\right\}$ of the stiffness subgraph, resulting in a weighted Laplacian. Since $M$ in (6.3) is a diagonal positive definite inertia matrix, it is invertible. Hence, (6.3) can be

\footnotetext{
${ }^{2}$ Note that the enumeration of the edges determines the order of the columns inside the incidence matrix $B_{\mathfrak{G}}$, which can be chosen freely. The Laplacian is independent of this column order.
} 
written in matrix form, similar to (6.2), as:

$$
\begin{aligned}
W_{v} \dot{x}(t)=\left[\begin{array}{cc}
0_{n \times n} & I_{n \times n} \\
-L_{\mathfrak{G} K}(K) & 0_{n \times n}
\end{array}\right] x(t) & +\left[\begin{array}{c}
0_{n \times m_{u}} \\
B_{\mathfrak{G} u}
\end{array}\right] u(t) \\
& +\left[\begin{array}{c}
0_{n \times m_{d}} \\
B_{\mathfrak{G} d}
\end{array}\right] d(t),
\end{aligned}
$$

where the state $x(t)$ is given by:

$$
x(t):=\left[\begin{array}{c}
\bar{x} \\
\dot{\bar{x}}
\end{array}\right]:=\left[\begin{array}{c}
x_{1} \\
x_{2} \\
x_{3} \\
\dot{x}_{1} \\
\dot{x}_{2} \\
\dot{x}_{3}
\end{array}\right]:=\left[\begin{array}{l}
x_{1} \\
x_{2} \\
x_{3} \\
x_{4} \\
x_{5} \\
x_{6}
\end{array}\right] \in \mathbb{R}^{2 n}
$$

and $W_{v} \in \mathbb{R}^{2 n \times 2 n}$ is a weights matrix incorporating the inertia matrix $M$ by

$$
W_{v}:=\left[\begin{array}{cc}
I_{n \times n} & 0_{n \times n} \\
0_{n \times n} & M
\end{array}\right] .
$$

Therefore, the dynamics can now be written as:

$$
\begin{aligned}
& \dot{x}(t)= \\
& W_{v}^{-1}\left[A(K) x(t)+\left[\begin{array}{c}
0_{n \times m_{u}} \\
B_{\mathfrak{G} u}
\end{array}\right] u(t)+\left[\begin{array}{c}
0_{n \times m_{d}} \\
B_{\mathfrak{G} d}
\end{array}\right] d(t)\right] \\
& \quad=F(K) x(t)+G_{u} u(t)+G_{d} d(t)
\end{aligned}
$$

\subsubsection{Variable stiffness actuator}

The strength of VSAs is that their stiffness $K$ can be changed at the same time as their position, which means that the stiffness is not a parameter in the way it was treated before, but is a state of the system that can be controlled by some control input. This situation is shown in Figure 6.3 (note the variable elastic element). Therefore, the state space should include a state variable representing the stiffness $K$ :

$$
x(t):=\left[\begin{array}{c}
\bar{x} \\
\dot{\bar{x}} \\
K
\end{array}\right]:=\left[\begin{array}{c}
x_{1} \\
x_{2} \\
x_{3} \\
\dot{x}_{1} \\
\dot{x}_{2} \\
\dot{x}_{3} \\
K
\end{array}\right]:=\left[\begin{array}{c}
x_{1} \\
x_{2} \\
x_{3} \\
x_{4} \\
x_{5} \\
x_{6} \\
x_{7}
\end{array}\right] \in \mathbb{R}^{2 n+m_{K}} .
$$

Control input $u(t)$ now also includes stiffness control, i.e., $u(t):=\left[\begin{array}{ll}u_{1}(t) & u_{2}(t)\end{array}\right]^{T}$, where $u_{1}(t)$ is the force input as before and $u_{2}(t):=\dot{K}$. The nonlinear dynamics are now:

$$
\begin{aligned}
\dot{x}(t) & =\left[\begin{array}{cc}
F(x(t)) & 0_{2 n \times m_{K}} \\
0_{m_{K} \times 2 n} & 0_{m_{K} \times m_{K}}
\end{array}\right] x(t) \\
& +\left[\begin{array}{cc}
G_{u} & 0_{2 n \times m_{K}} \\
0_{m_{K} \times m_{u}} & I_{m_{K} \times m_{K}}
\end{array}\right] u(t)+\left[\begin{array}{c}
G_{d} \\
0_{m_{K} \times m_{d}}
\end{array}\right] d(t) .
\end{aligned}
$$




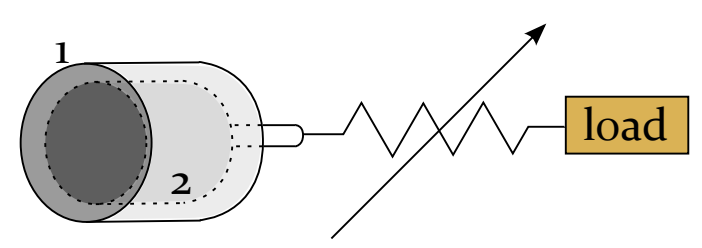

(a) Variable stiffness actuator.

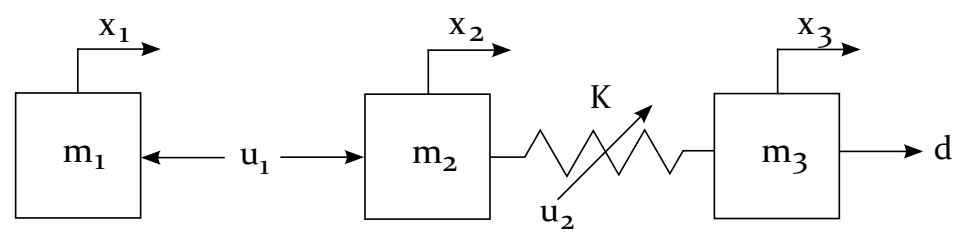

(b) Idealized physical model of a VSA.

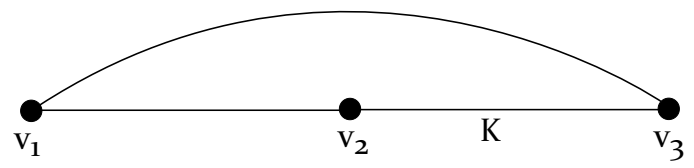

(c) Undirected graph $\mathfrak{G}$ of a VSA.

Figure 6.3: Schematic representation of a variable stiffness actuator (a), together with the idealized physical model (b), and the proposed graph model (c).

Obviously, like the SEA, $m_{K}=m_{d}=1$. Thus, a nonlinear affine dynamical system is obtained:

$$
\dot{x}(t)=f(x(t))+g(\cdot) v(t),
$$

where $g(\cdot)$ incorporates the matrices of the second and third terms in (6.5) and $v(t):=$ $\left[\begin{array}{ll}u(t) & d(t)\end{array}\right]^{\mathrm{T}}$.

\subsection{Optimal Stiffness Distribution}

The modeling method shown in Section 6.2 provides a modular way of composing multiple degrees of freedom systems. Modeling a system with many degrees of freedom is done in a straightforward manner by implementing an incidence matrix. In this section, it is shown that the stiffness setting or distribution can be optimized when using a multiple degrees of freedom compliant system. This complex model is used to show the straightforward scaling to models with many degrees of freedom, i.e., the modularity of the proposed method is made explicit.

To optimize the stiffnesses, the iterative Linear Quadratic Regulation (iLQR) [88] method is used, as it has been shown that it is well suited for the control of compliant actuators and manipulators [78, 88, 89] and is more efficient than comparative control methods. Although the iLQR method is able to handle high order cost functions by quadratizing them, the cost function that is considered here is indeed the well known quadratic form:

$$
\begin{aligned}
& J=\left(x(T)-x_{d}(T)\right)^{\mathrm{T}} S_{x}\left(x(T)-x_{d}(T)\right) \\
& +\int_{0}^{\mathrm{T}}\left(x(t)-x_{d}(t)\right)^{\mathrm{T}} Q_{x}\left(x(t)-x_{d}(t)\right)+u(t)^{\mathrm{T}} Q_{u} u(t) \mathrm{d} t,
\end{aligned}
$$

where $x(t)$ is the system state, $x_{d}(t)$ is the desired state trajectory, $u(t)$ is the control input, $S_{x} \geq 0$ is the constant matrix weighting state deviations at final time $T, Q_{x} \geq 0$ is the constant 
running cost weight matrix on the state deviation and $Q_{u}>0$ is the constant weight matrix on control input $u(t)$. This cost function encodes the control objective by including the desired state trajectory $x_{d}(t)$ that may be tracked. If only an end-time setpoint is desired, $x_{d}(t)=$ $0 \forall t<T$ and $x_{d}(T)=x_{d, T}$. For specific tasks, more terms may be introduced in the cost function to penalize or reward certain variables. For more information on optimal control, refer to Appendix C.

\subsubsection{System model}

Figure 6.4 shows a multiple degrees of freedom system with seven VSAs and four inertial loads (in this case represented as rotational loads). In the complete graph model $\mathfrak{G}$, there are $n=12$ vertices and $m=15$ edges, as shown in Figure 6.5. The numbers in Figure 6.4 indicate the VSAs used in the model. VSA-1, VSA-5 and VSA-7 are connected to the fixed world, represented by vertex $v_{1}$. Coordinates $q_{1}, q_{2}, q_{3}$ and $q_{4}$ are associated to the four inertial loads that are represented by vertices $v_{3}, v_{5}, v_{7}$ and $v_{9}$. Vertices $v_{2}, v_{4}, v_{6}, v_{8}, v_{10}, v_{11}$ and $\mathrm{v}_{12}$ are the rotors of the VSAs. Note that the stator of VSA-2 is rigidly connected to the inertial load with coordinate $q_{1}$, which are represented together by vertex $\mathrm{V}_{3}$. The stator of VSA-3 is in turn rigidly connected to the inertial load with coordinate $q_{2}$, and the same holds for VSA-4 and VSA-5. Vertex $v_{i}$ is assumed to have mass $m_{i}$, i.e., $m_{1}=10^{6}$ and $m_{2 \ldots 12}=1$, and disturbance force on load $q_{4}$ is $d(t)=0$. Since there are seven actuators to drive four loads, the system is redundant with respect to its control input. In particular, VSA-5 and VSA6 are used as biarticular actuators, which deliver power between two loads that are not direct neighbors, i.e., they deliver power between the fixed world and the load with coordinate $q_{2}$ and between load $q_{2}$ and $q_{4}$, respectively. This is in contrast with VSA-1, VSA-2, VSA-3 and VSA-4 (uniarticular actuators), which deliver power between neighboring loads. Moreover, VSA-7 is used as triarticular actuator, which delivers power between the fixed world and the load with coordinate $q_{3}$.

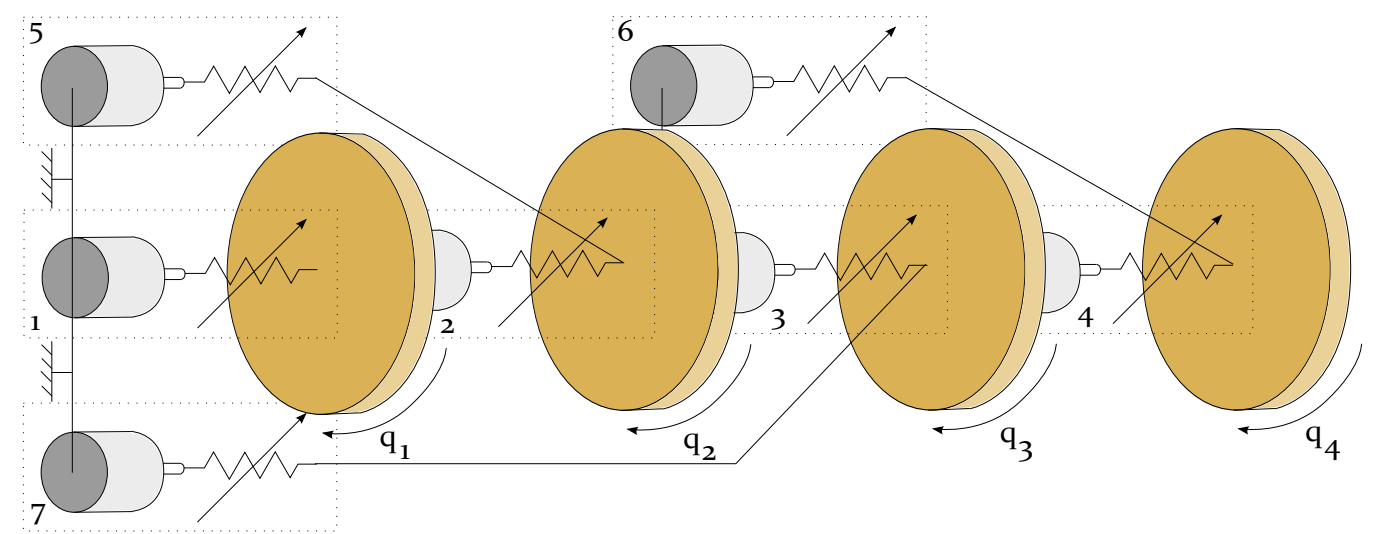

Figure 6.4: Schematic representation of the system with four sequential VSAs (number 1, 2, 3 and 4), two biarticular VSAs (number 5 and 6) and a triarticular actuator (number 7), driving four inertial loads with coordinates $q_{1}$, $q_{2}, q_{3}$ and $q_{4}$.

Similar to Figure 6.2, the subgraphs of this system can be formed and the incidence ma- 


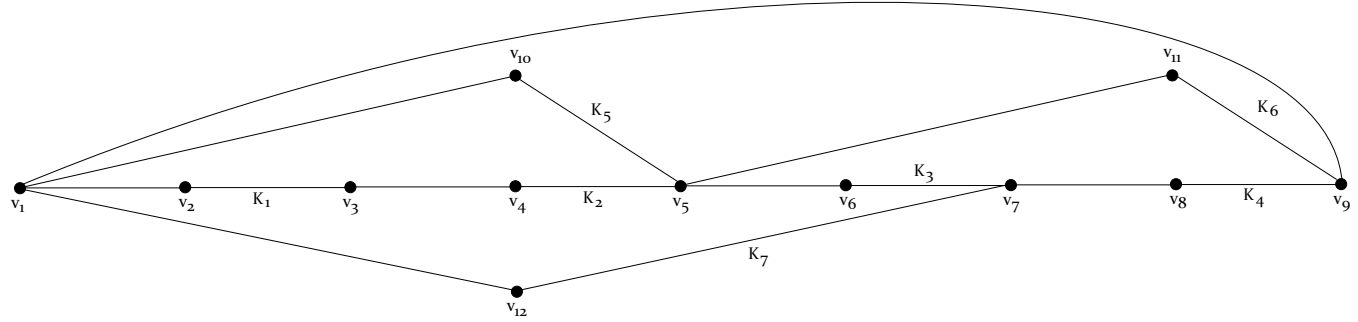

Figure 6.5: Graph of the schematic system of Figure 6.4.

trices can be set up, while enumerating the edges in the order of the VSAs:

$$
\begin{aligned}
B_{\mathfrak{G} u} & =\left[\begin{array}{cccccccccccc}
-1 & 1 & 0 & 0 & 0 & 0 & 0 & 0 & 0 & 0 & 0 & 0 \\
0 & 0 & -1 & 1 & 0 & 0 & 0 & 0 & 0 & 0 & 0 & 0 \\
0 & 0 & 0 & 0 & -1 & 1 & 0 & 0 & 0 & 0 & 0 & 0 \\
0 & 0 & 0 & 0 & 0 & 0 & -1 & 1 & 0 & 0 & 0 & 0 \\
-1 & 0 & 0 & 0 & 0 & 0 & 0 & 0 & 0 & 1 & 0 & 0 \\
0 & 0 & 0 & 0 & -1 & 0 & 0 & 0 & 0 & 0 & 1 & 0 \\
-1 & 0 & 0 & 0 & 0 & 0 & 0 & 0 & 0 & 0 & 0 & 1
\end{array}\right]^{\mathrm{T}} \\
B_{\mathfrak{G} K}= & {\left[\begin{array}{cccccccccccc}
0 & -1 & 1 & 0 & 0 & 0 & 0 & 0 & 0 & 0 & 0 & 0 \\
0 & 0 & 0 & -1 & 1 & 0 & 0 & 0 & 0 & 0 & 0 & 0 \\
0 & 0 & 0 & 0 & 0 & -1 & 1 & 0 & 0 & 0 & 0 & 0 \\
0 & 0 & 0 & 0 & 0 & 0 & 0 & -1 & 1 & 0 & 0 & 0 \\
0 & 0 & 0 & 0 & 1 & 0 & 0 & 0 & 0 & -1 & 0 & 0 \\
0 & 0 & 0 & 0 & 0 & 0 & 0 & 0 & 1 & 0 & -1 & 0 \\
0 & 0 & 0 & 0 & 0 & 0 & 1 & 0 & 0 & 0 & 0 & -1
\end{array}\right]^{\mathrm{T}} } \\
B_{\mathfrak{G} d}= & {\left[\begin{array}{ccccccccccccc}
-1 & 0 & 0 & 0 & 0 & 0 & 0 & 0 & 1 & 0 & 0 & 0
\end{array}\right]^{\mathrm{T}} . }
\end{aligned}
$$

Since there are now seven VSAs in the model, there are also seven elastic elements $\left(m_{K}=7\right)$. Therefore, the scalar elastic coefficient $K$ of (6.4) now becomes vectorial:

$$
K=\left[K_{1} \ldots K_{7}\right]^{\mathrm{T}} \text {. }
$$

The Laplacian for this model is given by $L_{\mathfrak{G} K}(K)=B_{\mathfrak{G} K} W_{\mathfrak{G} K} B_{\mathfrak{G} K}^{\mathrm{T}}$, where $W_{\mathfrak{G} K}:=\operatorname{diag}(K)$. The equations can now be formed, similar to (6.5), where state $x(t)$ is:

$$
x(t):=\left[\begin{array}{c}
\bar{x} \\
\overline{\bar{x}} \\
K
\end{array}\right]:=\left[\begin{array}{c}
x_{1} \\
\vdots \\
x_{12} \\
\dot{x}_{1} \\
\vdots \\
\dot{x}_{12} \\
K
\end{array}\right]:=\left[\begin{array}{c}
x_{1} \\
\vdots \\
x_{31}
\end{array}\right] \in \mathbb{R}^{2 n+m_{K}} .
$$

\subsubsection{Control}

For the first simulation, a resonating behavior on the last load with state $x_{9}(t)$ is desired:

$$
\begin{aligned}
x_{d, 9}(t) & =A \sin (\omega t+\phi) \\
x_{d, 21}(t) & =\dot{x}_{d, 9}(t)=\omega A \cos (\omega t+\phi),
\end{aligned}
$$


where $A=1$ is the amplitude, $\omega=\pi \mathrm{rad} / \mathrm{s}$ is the angular frequency and $\phi=0 \mathrm{rad}$ is the phase shift. All other state elements are unconstrained, but unbounded position and velocity growth is disallowed (which may result from corresponding weights equal to zero). Therefore, $x_{d, i}(t)=0$ where $i \neq\{9,21\}$ was used, while the weights are:

$$
\begin{aligned}
S_{x} & =10 \cdot I_{15 \times 15}, \text { but where } \\
S_{x}(9,9) & =S_{x}(21,21)=10^{4} \\
S_{x}(25,25) & \ldots S_{x}(31,31)=0 \\
Q_{x} & =S_{x}, Q_{u}=10^{2} \cdot I_{14 \times 14} .
\end{aligned}
$$

States $x_{9}$ and $x_{21}$ have large weights, so the controller is much less sensitive to the other state deviations from their desired value 0 . A deviation of states $x_{25 . .31}$ is not weighted, so the controller is indifferent to their value. Moreover, state deviations of $x_{9}$ and $x_{21}$ are penalized more than the control input, to ensure that the desired state trajectory is realized.

For the second simulation, only one period of a harmonic oscillation is desired for $x_{9}(t)$, starting at $t=t_{0}=5 \mathrm{~s}$ :

$$
\left.\begin{array}{l}
x_{d, 9}(t)=A-A \cos \left(\omega\left(t-t_{0}\right)+\phi\right) \\
x_{d, 21}(t)=\omega A \sin \left(\omega\left(t-t_{0}\right)+\phi\right)
\end{array}\right\} \quad \begin{gathered}
\text { for } \\
t_{0} \leq t \leq t_{0}+\frac{2 \pi}{\omega},
\end{gathered}
$$

where, again, the parameters are the same as before. For $t_{0}>t>t_{0}+\frac{2 \pi}{\omega}, x_{d, 9}(t)=x_{d, 21}(t)=$ 0 . This can be thought of as a back and forth motion of the last load. The cost function weights are identical to (6.8).

\subsubsection{Simulations and results}

The first simulation result can be seen in Figure 6.6, in which the partial state and control vectors are plotted (the unplotted states show similar behavior). The rows represent, from top to bottom: position, actuator stiffness and control input. It is shown that load state $x_{9}(t)$ converges to its desired trajectory, while the other position states are as close to their desired states as possible (resulting in small internal resonance behavior of the system). The stiffnesses of VSA-4 and VSA- 6 clearly converge to a constant value, while control input $u(t)$ approaches zero, which means that the system is steered to the resonance behavior.

The second simulation result is shown in Figure 6.7. It can be seen that load state $x_{9}(t)$ converges to its desired trajectory (and again resulting in internal resonance behavior of other states). Again, the stiffnesses of the VSAs are adapted during the task and it is observed that the stiffness of VSA-4 and VSA- 6 show the most considerable change. Hence, the result of these simulations is that an optimal stiffness distribution to achieve a task is found. These results also show that proper tuning of the compliance at the load on which a task is imposed is most important.

\subsection{Discussion}

This chapter has presented the modeling of compliant systems on graphs. This modeling framework is modular in the sense that it allows easy scaling to systems with more degrees of freedom, since modeling a mechanism only requires the use of an incidence matrix. Hence, the complexity does not increase considerably. The traditional way of modeling may be to construct free body diagrams, summing forces on the masses and using Newton's second law 
of motion to obtain the same set of equations. This is cumbersome for systems with many degrees of freedom, which makes the advantage of the proposed method clear.

It is shown that for the oscillation simulation, the stiffness is adapted to a certain steadystate preferred value as to steer the system towards resonance. During steady-state oscillation, the stiffness is not adapted anymore, which is indeed expected for a single frequency resonance behavior. This means that a VSA would not be necessary for this task and, therefore, a SEA would suffice in this particular situation. In case of the back and forth simulation, it is shown that the stiffness of two VSAs was changed during the motion, while the stiffness of the other VSAs was not changed considerably. This indicates that a VSA is beneficial at certain locations in these models, but not necessary for the actuation at other locations where, hence, a SEA can perform equally well. Furthermore, it was shown that proper tuning of the compliance at the load on which a task is imposed is most important, and that the compliance in the rest of the system is less significant. Therefore, the methodology laid out in this chapter can be exploited to find an optimal stiffness distribution of a compliant mechanism for a given task, and can assist in making design decisions.

\subsection{Conclusion}

In this chapter, the graph theoretic models of a series elastic actuator, a variable stiffness actuator and a compliant system with multiple variable stiffness actuators have been presented. Graph theoretic analyses ensure a structured and modular way of describing a system and allow a straightforward extension to more complex systems. The iLQR optimal controller was used to impose a certain task on their outputs and to find the optimal stiffness distribution. A simulation of the behavior of a system with multiple VSAs showed that the controller steers the system to its resonance behavior and showed that only SEAs are required. For a back and forth motion of that system, it was shown that stiffness adaptation is beneficial for reducing the cost while meeting the objective. However, those results also indicate that a particular actuator need not necessarily be a VSA, which indicates the possibility of finding an optimal stiffness distribution of complex robotic systems using this approach. 


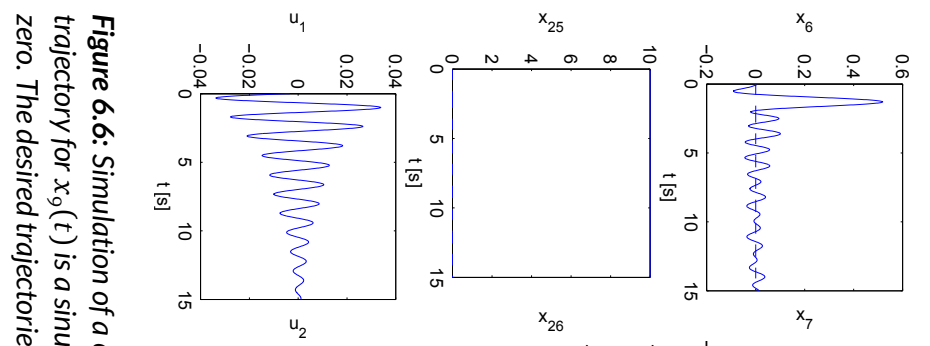

के. ज्ञ

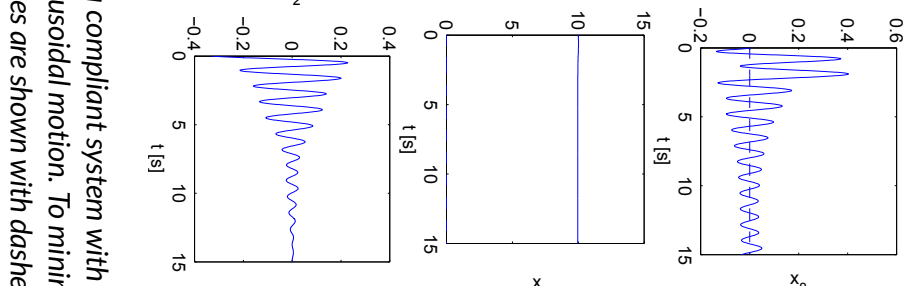

约 ․․․․

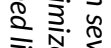

第索

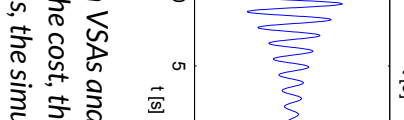
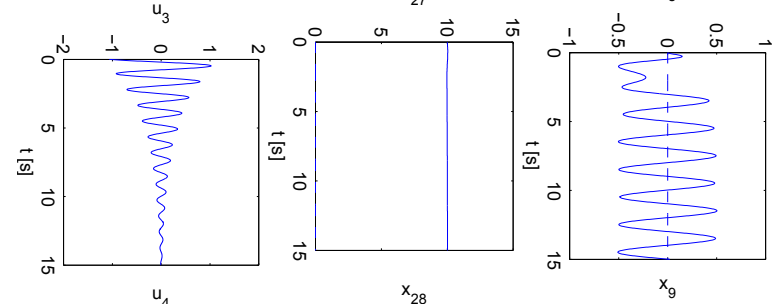

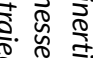

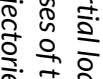

की

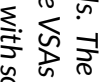

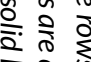

곤

$\stackrel{\frac{9}{0}}{\frac{1}{3}}$
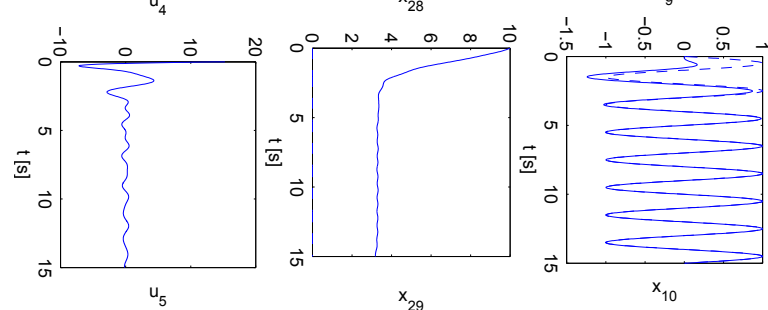

$\frac{1}{0} \frac{1}{0}$

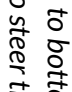
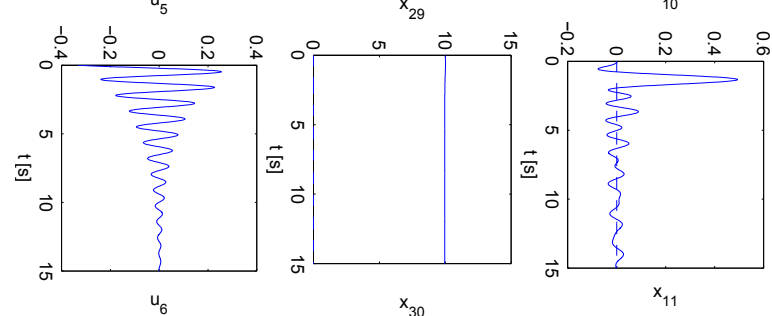

施 $\frac{0}{2}$

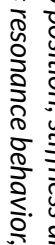
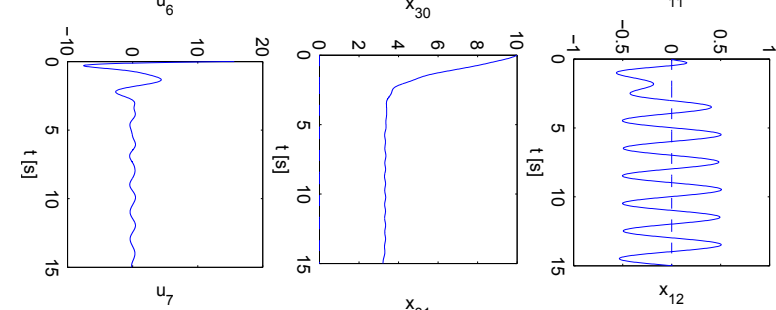

‥ 을

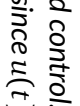

운

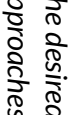
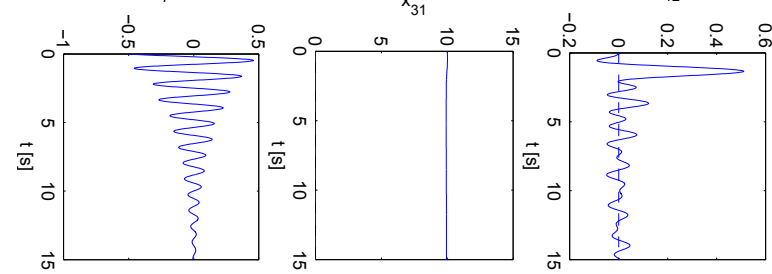

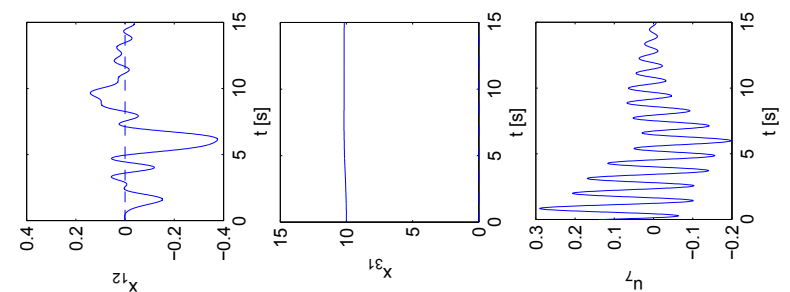

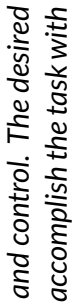
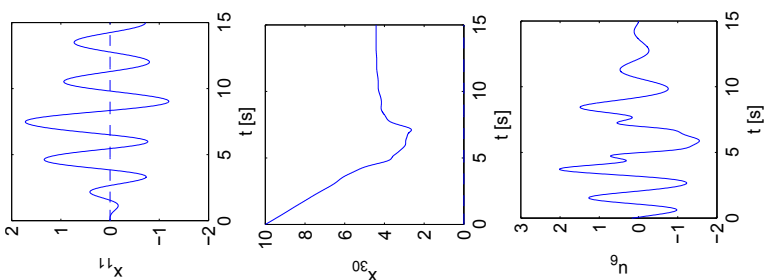

ญㅇ

능
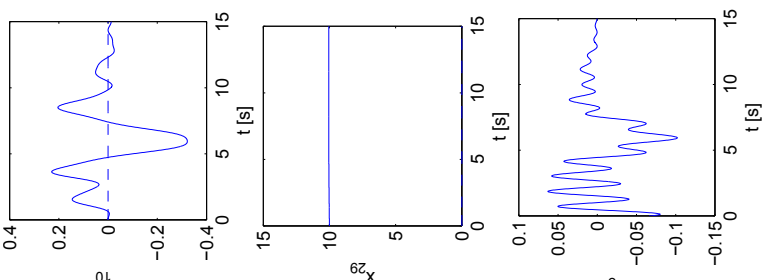

늠

范

$\stackrel{\text { : }}{\leftrightarrows}$

8

है

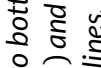

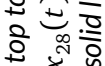
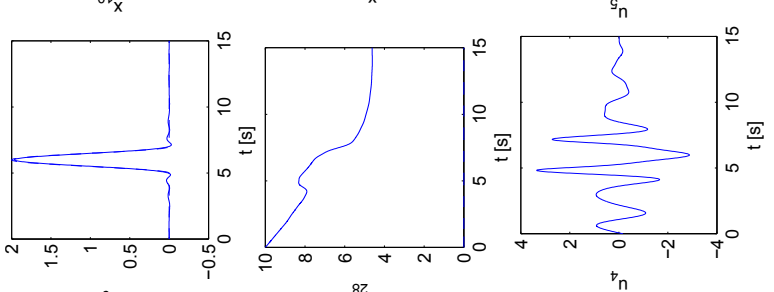

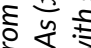

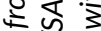
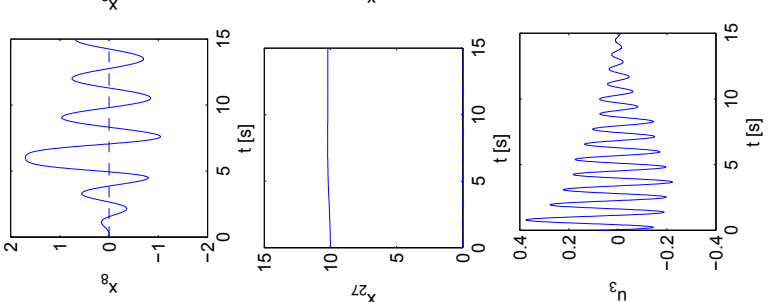

$\pm \leftleftarrows$

‡ั

눙 ญ

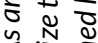

है है

वे है

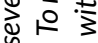
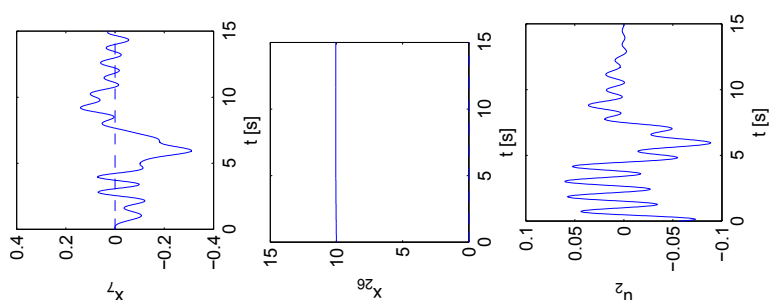

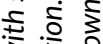

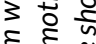

है है थे

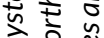

站

ơ

है 总

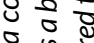

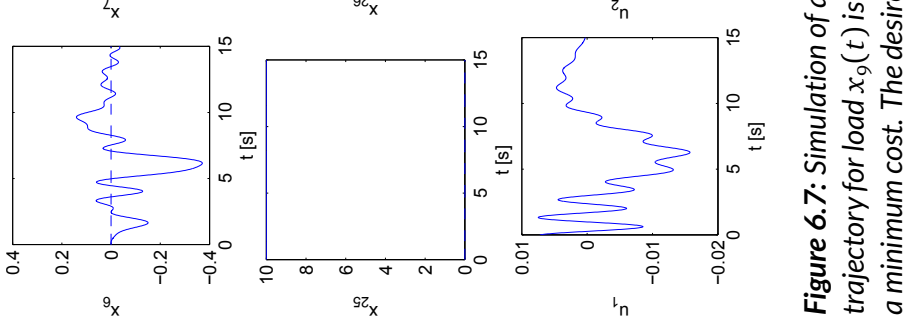


CHAPTER 7

\section{Compliant Robotic Manipulators on Graphs}

This chapter proposes a modeling method for generic compliant robotic manipulators. It is based on graph theory and the port-Hamiltonian formalism, which allows a modular approach to the interconnection of rigid bodies with compliant actuators by means of kinematic pairs. This modularity enables to simply and straightforwardly adapt the model when a manipulator's actuator morphology is changed. An example of a spatial three degrees of freedom manipulator shows that this modeling method is more suitable for modeling changes in actuator placement than the traditional Euler-Lagrange method.

This chapter has been submitted for possible publication as:

[90] S. S. Groothuis, S. Stramigioli, and R. Carloni, "Modeling robotic manipulators powered by variable stiffness actuators: a graph-theoretic and port-hamiltonian formalism," IEEE Transactions on Robotics, 2016. Submitted

A more preliminary version has been previously published as:

[91] S. Groothuis, S. Stramigioli, and R. Carloni, "Compliant manipulators on graphs," in Proceedings of the IEEE/RSJ International Conference on Intelligent Robots and Systems, 2015.

(c) 2015 IEEE 
CURRENT models of compliant manipulators, possibly powered by variable stiffness actuators [92], are based on traditional modeling techniques as presented, for instance, in $[93,94]$, and [95]. These models, which have their roots at the Euler-Lagrange equations, are widely used because of their suitability in control design. However, these models are not very flexible with respect to changes in the system, since a change in, for instance, the kinematic structure or actuator placement basically requires reanalyzing the complete system. This holds, for instance, for the manipulator with two different articulation types $([96,97])$, namely uniarticulation and biarticulation, that is shown in Figure 7.1.

Therefore, graph-theoretic modeling has been applied to the modeling of robotic systems. It allows to describe low-dimensional systems as well as high(er)-dimensional (and more complex) systems in an identical way, since a graph captures the topology of a system and embodies physical interconnection laws. This makes the model inherently modular. A graph theoretic description of a VSA was presented in Chapter 6 [77], where the application of the laws imposed by graph theory leads to a one-dimensional Euler-Lagrange model. A one-dimensional system can properly be described using that approach, as was also shown in [98], but when the model is extended to higher dimensions, the complexity increases since two separate graphs are necessary, i.e., one for rotations and one for translations, like is done in $[99,100,101,102,103,104,105]$, and [106]. The distinction between rotation and translation is not necessary when the port-Hamiltonian modeling framework is adopted, that allows the description of generalized rigid body motions, of which its structure and interconnection can be described by graph theory. This was shown in [85], where mainly massspring-damper systems were treated and the extension to spatial mechanisms was briefly introduced. One graph then suffices to describe the instantaneous rigid body's movement as a twist, which can be related to a screw motion [107]. Moreover, the port-based modeling of the port-Hamiltonian formalism, contrary to Euler-Lagrange modeling, naturally allows the interconnection of several systems through power conjugated effort and flow variables associated to power ports. In [82], this was used for the modeling of rigid robotic mechanisms and mechanisms with passive compliant elements.

Here, the work in Chapter 6 [77], that considered a one-dimensional Euler-Lagrange model, and previous work in [91], in which the model of a compliant robotic manipulator in the port-Hamiltonian modeling framework was presented, is extended and formalized. The model of a port-Hamiltonian generic compliant actuator (a VSA), described in graph theory, is proposed. It is elaborated that the usage of two kinematic pairs per compliant joint is necessary. A graph model for generic robotic manipulators which decouples the manipulator's kinematic structure from the actuators' connection topology is presented, which means that the articulation type of the generic compliant actuators can be easily defined on the graph model. In this way, a VSA is not fixed to a certain joint or in a certain configuration a priori, but it is a modular entity that can be connected as desired by changing the topology of the system, given by a mapping that the graph defines.

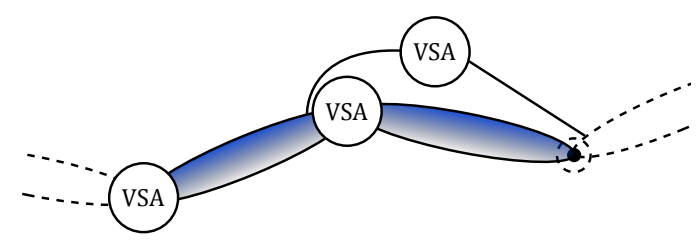

Figure 7.1: The inclusion of biarticulation actuators (rightmost VSA) in robotic systems is not common due to the increased system complexity. 
In order to be able to present the model, Section 7.1 elaborates first on the basics of portHamiltonian systems and graph theory. The model is then explained in Section 7.2 and its strength is made explicit by treating an elaborate example and comparing it to a traditional Euler-Lagrangian model in Section 7.3. The model and its results are discussed in Section 7.4 and the chapter concludes with Section 7.5.

\subsection{Port-Hamiltonian Systems and Graph Theory}

This section summarizes the existing concepts from port-Hamiltonian systems and graph theory that are instrumental in the modeling of compliant robotic manipulators.

\subsubsection{Port-Hamiltonian systems}

A port-Hamiltonian system is given by:

$$
\begin{aligned}
& \dot{x}=J(x) \frac{\partial H(x)}{\partial x}+g(x) u \\
& y=g^{T}(x) \frac{\partial H(x)}{\partial x},
\end{aligned}
$$

with Hamiltonian energy function $H(x)$, skew-symmetric structure matrix $J(x)=-J^{\mathrm{T}}(x)$, local coordinates $x$ on manifold $\mathscr{X}$, input matrix $g(x)$, and collocated port variables $u$ and $y$. The change in the system's stored energy is described by the power balance, which, because of the skew-symmetry of $J(x)$, is given by:

$$
\begin{aligned}
\frac{d}{d t} H(x)=\frac{\partial^{\mathrm{T}} H(x)}{\partial x} \frac{d x}{d t} & =\frac{\partial^{\mathrm{T}} H(x)}{\partial x}\left(J(x) \frac{\partial H(x)}{\partial x}+g(x) u\right) \\
& =\frac{\partial^{\mathrm{T}} H(x)}{\partial x} J(x) \frac{\partial H(x)}{\partial x}+\frac{\partial^{\mathrm{T}} H(x)}{\partial x} g(x) u=y^{\mathrm{T}} u,
\end{aligned}
$$

stating that the energy change equals the supplied power through port $\langle y, u\rangle$. A specific case of the system in (7.1) is obtained, if a distinction is made between explicit ports, i.e. storage, control, interaction, and dissipation ports, to which energy flows are associated. Such an input-state-output port-Hamiltonian system, with control and interaction port, is given by:

$$
\begin{aligned}
& \dot{x}=[J(x)-R(x)] \frac{\partial H(x)}{\partial x}+g(x) u+h(x) d \\
& y=g^{T}(x) \frac{\partial H(x)}{\partial x} \\
& z=h^{T}(x) \frac{\partial H(x)}{\partial x}
\end{aligned}
$$

where $x=\left[x_{1}, \ldots, x_{k}\right]^{\mathrm{T}}=\left[q_{1}, \ldots, q_{i}, p_{1}, \ldots, p_{j}\right]^{\mathrm{T}}$ is the $k$-dimensional vector of elements of the state manifold $\mathscr{X}, J(x)$ is the $k \times k$ skew-symmetric structure matrix, i.e. $J(x)=-J^{\mathrm{T}}(x)$, defining the interconnection between efforts and flows, $R(x) \geq 0$ is the $k \times k$ symmetric dissipative structure matrix, $g(x)$ is the $k \times r$ input matrix, $u \in \mathbb{R}^{r}$ is the vector of the generalized control inputs, $h(x)$ is the $k \times w$ interaction matrix, $d \in \mathbb{R}^{w}$ is the vector of generalized interaction inputs, $y \in \mathbb{R}^{r}$ is the generalized control output vector collocated with control input $u$ and $z \in \mathbb{R}^{w}$ is the generalized interaction output vector collocated with interaction input $d$. Note that $y^{\mathrm{T}} u$ is the power flow through the control port and $z^{\mathrm{T}} d$ is the power flow through the interaction port. A power-continuous Dirac structure $(\mathscr{D})$ connects the ports and defines the power flow between them, as given by $J(x)$. Refer to Appendix B for more information. 


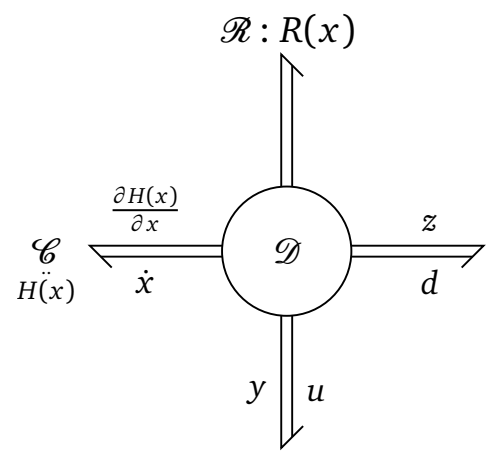

Figure 7.2: Graphic representation of a generic port-Hamiltonian model, where the ports are, clockwise and starting from the top port: dissipation port, interaction port, control port, and storage port. The power-continuous Dirac structure $(\mathscr{D})$ connects the ports and defines the power flow.

\subsubsection{Boundary and internal graph vertices}

A directed graph $\mathfrak{G}$ is defined by a set of $n$ vertices $\mathbb{V}_{\mathfrak{G}}=\left\{\mathrm{V}_{1}, \mathrm{~V}_{2}, \ldots, \mathrm{V}_{n}\right\}$ and a set of $m$ directed edges $\mathbb{E}_{\mathfrak{G}}=\left\{\mathrm{E}_{1}, \mathrm{E}_{2}, \ldots, \mathrm{E}_{m}\right\} \subseteq \mathbb{V}_{\mathfrak{G}} \times \mathbb{V}_{\mathfrak{G}}$, i.e., a set of ordered pairs of vertices defined by the Cartesian product $\times$, which defines the connections between the vertices. An undirected graph consists of edges that are unordered pairs of vertices. The relevant graph matrices have been treated in Section 6.1.

It is possible to distinguish between boundary and internal vertices of a graph [106]. Define the set $\mathbb{N}_{\mathfrak{G}}\left(\mathrm{V}_{i}\right)$ of all neighboring vertices of vertex $\mathrm{V}_{i}$, i.e., vertices that are coupled to $\mathrm{v}_{i}$ by an edge. The distance $\mathrm{d}\left(\mathrm{v}_{1}, \mathrm{v}_{2}\right)$ between two vertices $\mathrm{v}_{1}, \mathrm{v}_{2} \in \mathrm{V}_{\mathfrak{G}}$ of graph $\mathfrak{G}$ is the length of a shortest path between vertex $v_{1}$ and $v_{2}$. A vertex $v_{1}$ is a boundary vertex of vertex $\mathrm{v}_{2}$, if no neighboring vertex $\mathrm{v}_{3} \in \mathbb{N}_{\mathfrak{G}}\left(\mathrm{v}_{1}\right)$ of $\mathrm{v}_{1}$ is further from $\mathrm{v}_{2}$ than $\mathrm{v}_{1}$. In other words: $a$ vertex $v_{1}$ is a boundary vertex of vertex $v_{2}$, if all neighboring vertices $v_{3} \in \mathbb{N}_{\mathfrak{G}}\left(v_{1}\right)$ of $v_{1}$ are closer to or of equal distance from $v_{2}$ than $v_{1}$. A vertex $v_{1}$ is a boundary vertex of graph $\mathfrak{G}$, if $\mathrm{v}_{1}$ is a boundary vertex of some vertex $\mathrm{V} \in \mathbb{V}_{\mathfrak{G}}$. Internal vertices are the vertices that are not boundary vertices. However, this definition is based on the topology of the graph, and can be relaxed in a physical system control context by stating that the boundary vertices are those vertices to which one chooses to apply, for instance, external forces. Either way, the set of vertices $\mathbb{V}_{\mathfrak{G}}$ can be split into a boundary vertex set $\mathbb{V}_{\mathfrak{G} b}$, which is a subset of the vertex set $\mathbb{V}_{\mathfrak{G}}$, and internal vertex set $\mathbb{V}_{\mathfrak{G} i}$, i.e.:

$$
\begin{aligned}
& \mathbb{V}_{\mathfrak{G} b} \subset \mathbb{V}_{\mathfrak{G}} \\
& \mathbb{V}_{\mathfrak{G} i}:=\mathbb{V}_{\mathfrak{G}} \backslash \mathbb{V}_{\mathfrak{G} b} .
\end{aligned}
$$

The boundary vertices are useful to describe systems that are open to interconnection to other systems.

\subsubsection{Port-Hamiltonian mechanical systems on graphs}

When port-Hamiltonian systems are applied to graphs, the coupling between the effort and flow variables is defined by the graph incidence matrix $B_{\mathfrak{G}}$ as presented in [85] and repeated here. If $\mathscr{E}_{f}$ denotes the space of edge flows (with dual space $\mathscr{E}_{e}$ denoting the edge efforts) and $\mathscr{V}_{f}$ denotes the space of vertex flows (with dual space $\mathscr{V}_{e}$ denoting the vertex efforts), then 
the mapping between the spaces is given by:

$$
\begin{aligned}
& B_{\mathfrak{G}}:=\mathscr{E}_{f} \mapsto \mathscr{V}_{f} \\
& B_{\mathfrak{G}}^{*}:=\mathscr{V}_{e} \mapsto \mathscr{E}_{e},
\end{aligned}
$$

where $*$ indicates the dual mapping. Following the previous reasoning on internal and boundary vertices, the incidence operator splits into two operators mapping between the internal and boundary spaces, i.e.,

$$
B_{\mathfrak{G}}=B_{\mathfrak{G} i} \oplus B_{\mathfrak{G} b}
$$

However, it depends on the physical nature of the boundary vertices how the boundary incidence map is defined. A distinction is made between boundary vertices with associated mass and massless boundary vertices. In the former case, the flow of an incident edge is mapped to the attached boundary vertex flow, while in the latter case, the effort of the incident edge is mapped to the attached boundary vertex effort.

In particular, these individual mappings, in the mass and massless boundary vertex case, are given by:

$$
\begin{aligned}
\text { mass: } & \text { massless: } \\
B_{\mathfrak{G} i}:=\mathscr{E}_{f} \mapsto \mathscr{V}_{f_{i}} & B_{\mathfrak{G} i}:=\mathscr{E}_{f} \mapsto \mathscr{V}_{f} \\
B_{\mathfrak{G} b}:=\mathscr{E}_{f} \mapsto \mathscr{V}_{f} & B_{\mathfrak{G} b}:=\mathscr{E}_{e} \mapsto \mathscr{V}_{e b} \\
B_{\mathfrak{G} i}^{*}:=\mathscr{V}_{e i} \mapsto \mathscr{E}_{e} & B_{\mathfrak{G} i}^{*}:=\mathscr{V}_{e i} \mapsto \mathscr{E}_{e} \\
B_{\mathfrak{G} b}^{*}:=\mathscr{V}_{e b} \mapsto \mathscr{E}_{e} & B_{\mathfrak{G} b}^{*}:=\mathscr{V}_{f b} \mapsto \mathscr{E}_{f}
\end{aligned}
$$

These mappings are non-gyrating mappings, i.e., mappings from flows to flows or from efforts to efforts. We present here the gyrating incidence mappings (indicated with a superscripted $\mathfrak{g}$ ) in a similar way, since these mappings are necessary in the port-Hamiltonian description of compliant actuators on graphs. Again, the distinction is made between boundary vertices with or without mass. The mappings are defined as:

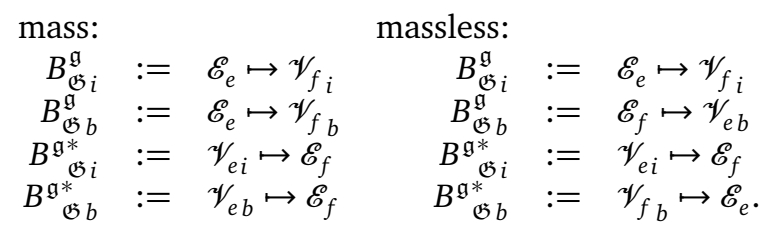

These map an element of one of the power variable spaces to an element of the other power variable in the dual space, e.g., edge effort to vertex flow, which is necessary in the description of a VSA on graphs.

\subsection{Compliant Manipulators on Graphs}

Compliant manipulators are defined and characterized here as rigid body mechanisms that are arbitrarily kinematically coupled and are actuated by compliant actuators.

\subsubsection{Port-Hamiltonian variable stiffness actuator on graphs}

A one-dimensional VSA is a generic compliant actuator capable of delivering power to an attached load while allowing potential energy storage through an adjustable storage element [38]. A schematic representation of a generic VSA is shown in Figure 7.3, where a motor with a rotor (1) and a stator (2), an adjustable elastic element and an attached load 


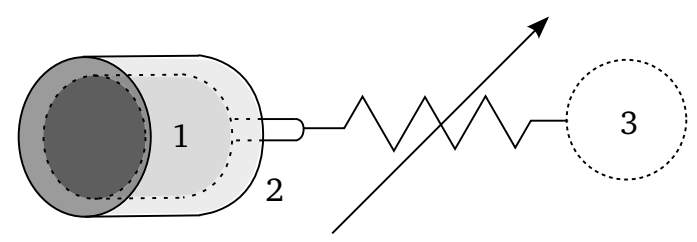

Figure 7.3: Schematic representation of a VSA, showing a rotor (1), a stator (2), an adjustable elastic element, and an attached load to the output (3).

(3) can be distinguished. Interaction with a VSA is possible through interaction with the output or interaction with the stator. Thus, interaction is associated to a two-dimensional interaction port with conjugated effort and flow variables $e_{\text {int }}$ and $f_{\text {int }}$, respectively. This is an extension of the work in [38] where a port-Hamiltonian model of a VSA was presented, but where it is assumed that the actuator acts with respect to an inertial reference (the stator is connected to the fixed world). Therefore, it has only a one-dimensional interaction port allowing physical interaction with the environment through the output only. This assumption does not hold when considering a serial connection of bodies in a manipulator, where the VSA interacts with bodies using both its stator (connected to a preceding body) and its output (connected to a subsequent body).

The internal rotor with mass $m$ stores kinetic energy, which is a function of the rotor's momentum $p$. Moreover, it is assumed that the elastic coefficient $k$ of the elastic element can be directly adjusted by some control $u_{k}$, which means that the change of stored potential energy in the elastic element is a function of both its elongation $s$ and its elastic coefficient $k$ (the VSA's output stiffness). This is also different from [38], in which the VSA's output stiffness is adjusted through other internal degrees of freedom.

The state of a generic VSA is given by the individual storage states:

$$
x:=\left[\begin{array}{l}
s \\
k \\
p
\end{array}\right],
$$

and the quadratic Hamiltonian of a VSA becomes:

$$
H(x):=H(s, k, p)=\frac{1}{2} k s^{2}+\frac{1}{2} \frac{p^{2}}{m} .
$$

Hence, the storage port is represented by a three-dimensional port $\left(\frac{\partial H(x)}{\partial x}, \dot{x}\right)$. The control port through which the VSA can be controlled, i.e., the force between the stator and rotor and the adjustment of the elastic coefficient, is represented by a two-dimensional port $(y, u)$.

Similarly to what has been shown in Chapter 6 [77] and in [91], the proposed undirected graph model of the VSA is shown in Figure 7.4, where three vertices and two edges can be distinguished. The middle vertex $v_{1}$ is the internal (rotor mass) vertex of the graph, shown with a solid circle, and the other two vertices $v_{2}$ and $v_{3}$ are the boundary vertices to which a mass can be associated, shown with open circles. In case of a VSA, the stator mass and output mass are associated to these boundary vertices. Figure 7.4b and Figure 7.4c show the directed input subgraph $\mathfrak{G}_{u}$ and stiffness subgraph $\mathfrak{G}_{k}$, respectively.

The incidence matrices of these subgraphs can be found and, following the reasoning in Section 7.1, they can be split in an internal and boundary part. For subgraph $\mathfrak{G}_{u}$, the incidence 


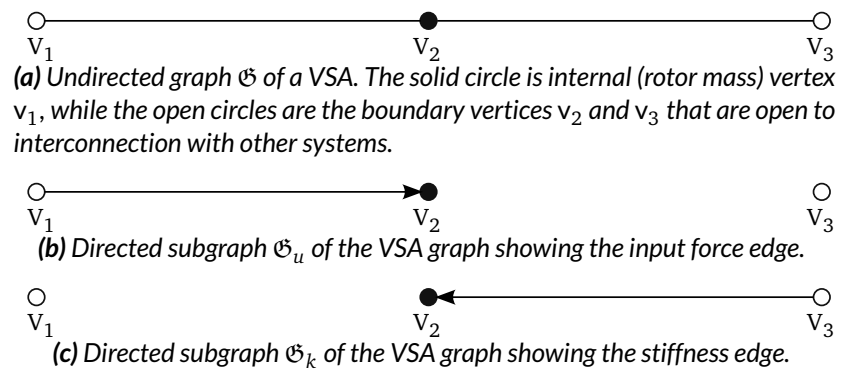

Figure 7.4: Proposed graph and subgraphs of a VSA.

matrix is given by:

$$
B_{\mathfrak{G} u}:=\left[\frac{B_{\mathfrak{G} u i}}{B_{\mathfrak{G} u b}}\right]=\left[\begin{array}{c}
1 \\
-1 \\
0
\end{array}\right] .
$$

In [91], the incidence matrix for the subgraph $\mathfrak{G}_{k}$ was non-gyrating, assuming that the physical domains that are coupled are the same. However, here the gyrating incidence matrix is used since formally two distinct physical domains are coupled. These domains are the potential domain (potential energy storage in the elastic element associated to the only edge in Figure 7.4c) and kinetic domain (kinetic energy storage in the internal rotor mass associated to $\mathrm{V}_{1}$ ). Hence, the (gyrating) incidence matrix is here given by:

$$
B_{\mathfrak{G} k}^{\mathfrak{g}}:=\left[\frac{B_{\mathfrak{G} k i}^{\mathfrak{g}}}{B_{\mathfrak{G} k b}^{\mathfrak{g}}}\right]=\left[\begin{array}{c}
1 \\
\hline 0 \\
-1
\end{array}\right] .
$$

The input-state-output port-Hamiltonian model as in (7.3) of a VSA, when choosing local coordinates $x$ according to (7.5) on state-manifold $\mathscr{X}$ [38], can now be written as:

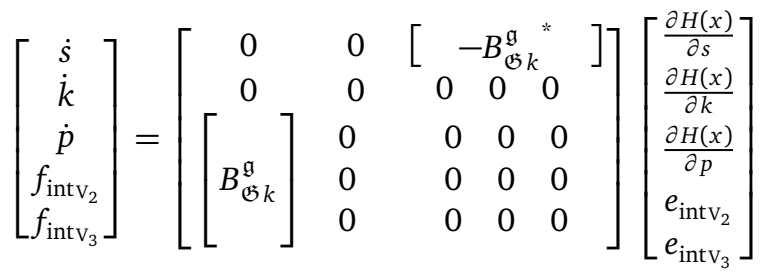

$$
\begin{aligned}
& +\left[\begin{array}{cc}
0 & 0 \\
0 & 1 \\
B_{\mathfrak{G} u} & 0 \\
& 0 \\
& 0
\end{array}\right]\left[\begin{array}{l}
u_{p} \\
u_{k}
\end{array}\right]
\end{aligned}
$$

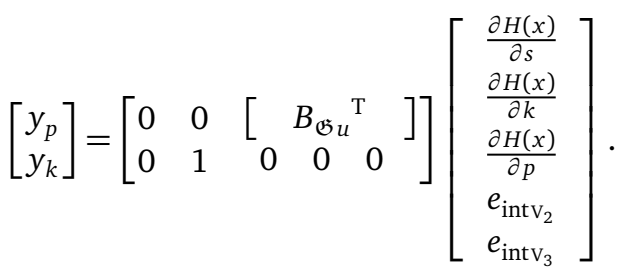




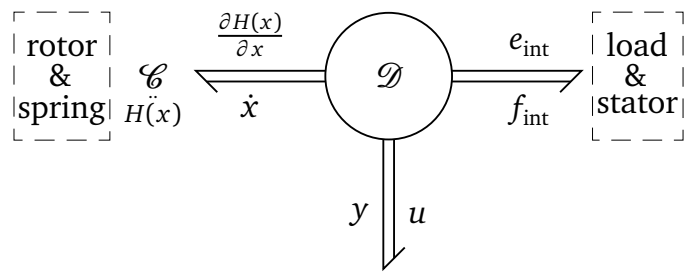

Figure 7.5: Visualization of a port-Hamiltonian model of a VSA, with storage port $\left(\frac{\partial H(x)}{\partial x}, \dot{x}\right)$, control port $(y, u)$, and interaction port $\left(e_{\text {int }}, f_{\text {int }}\right)$.

The two-dimensional interaction port with variables $\left(e_{\text {intv }_{2}}, f_{\text {intv }_{2}}\right)$ and $\left(e_{\text {intv }_{3}}, f_{\text {int }_{3}}\right)$, corresponding to vertices $v_{2}$ and $v_{3}$ in Figure 7.4, and to labels (2) and (3) in Figure 7.3, respectively, can be used to interact with the environment, where the flows $f_{\text {int }}$ represent the force on the boundary vertices and the efforts $e_{\text {int }}$ represent the velocities of the boundary vertices. Control port variables $\left(y_{p}, u_{p}\right)$ and $\left(y_{k}, u_{k}\right)$ are the conjugated power variables to the internal mass with momentum $p$ (the input force), and to the elastic element with elastic coefficient $k$ (the stiffness change), respectively.

This can be visualized as depicted in Figure 7.5, which shows the control port, interaction port and storage port being connected by a Dirac structure $\mathscr{D}$, which defines the power distribution between the connected ports.

\subsubsection{Kinematic structure}

In a kinematic structure, rigid bodies are interconnected through kinematic pairs. These kinematic pairs define the directions in which the bodies can move and, thus, constrain the relative motions between two bodies. ${ }^{1}$ The configuration of a rigid body $\Psi_{i}$ with respect to an observer $\Psi_{j}$ is defined by a homogeneous matrix $H_{j}^{i} \in \mathbb{R}^{4 \times 4}$, once a reference configuration has been chosen, and is an element of the Lie group $S E(3)$. Each element of this group represents a configuration of the rigid body relative to the chosen reference configuration. A motion as a function of time is described by a trajectory on this manifold parametrized by time. The instantaneous velocity at a certain time then lies in the tangent plane (a vector space) at a certain configuration on the manifold. This vector in the tangent plane can be mapped to a vector in the Lie algebra se(3) (the tangent space at the identity element of the Lie group). This is the space of relative instantaneous rigid body motions that can be shown to be the generalized relative screw motions. The dual to the Lie algebra $\left(s e^{*}(3)\right)$ is associated to the wrenches on the rigid bodies.

\section{Kinematic pair}

A one-port kinematic pair [107], as shown in Figure 7.6a, defines directions in which two connected bodies can move relative with respect to one another, in a subspace of $s e(3)$, while constraining the other directions to zero velocity. A two-port kinematic pair, as shown in Figure 7.6b, includes an additional power port through which power can be supplied by, for instance, motors. The motor force is then converted to a wrench that is applied to both connected bodies as an action and reaction, which is a fundamental property of a kinematic pair. A kinematic pair has associated free $(\mathrm{F})$ and constrained (C) twists and wrenches $\left(T_{\mathrm{F} \mid \mathrm{C}}\right.$ and $\left.W_{\mathrm{F} \mid \mathrm{C}}\right)$, that are elements of corresponding spaces $\left(\mathscr{T}_{\mathrm{F} \mid \mathrm{C}}\right.$ and $\left.\mathscr{W}_{\mathrm{F} \mid \mathrm{C}}\right)$, which in turn are subspaces

\footnotetext{
${ }^{1}$ Here, only holonomic kinematic constraints are considered.
} 
of the algebra $s e(3)$ and the dual algebra $s e^{*}(3)$, respectively. In general, the $l$ free twists of a kinematic pair

$$
{ }^{1} T_{\mathrm{F}}, \quad \ldots, \quad{ }^{l} T_{\mathrm{F}} \in \mathscr{T}_{\mathrm{F}} \subset \operatorname{se}(3)
$$

are known and can be defined. However, since there is no intrinsic metric on se(3) [108] defining an intrinsic complement, the $6-l$ constrained twists

$$
{ }^{1} T_{\mathrm{C}}, \quad \ldots, \quad{ }^{6-l} T_{\mathrm{C}} \in \mathscr{T}_{\mathrm{C}} \subset \operatorname{se}(3)
$$

cannot be uniquely defined. What can be defined using the free twists are the $6-l$ constrained wrenches

$$
{ }^{1} W_{\mathrm{C}}, \quad \ldots, \quad{ }^{6-l} W_{\mathrm{C}} \in \mathscr{W}_{\mathrm{C}} \subset s e^{*}(3)
$$

The constrained wrenches are those wrenches that cause no acceleration of the involved bodies and cause no power transfer in the direction of the free twist, i.e.:

$$
{ }^{i} W_{\mathrm{C}}{ }^{j} T_{\mathrm{F}}=0 \forall i, j .
$$

Moreover, there is also no unique definition to map free twists to corresponding free wrenches, since a free twist may be generated by many different wrenches. Therefore, the free wrenches are a choice made by placing actuators. By having defined $l$ free wrenches

$$
{ }^{1} W_{\mathrm{F}}, \quad \ldots, \quad{ }^{l} W_{\mathrm{F}} \in \mathscr{W}_{\mathrm{F}} \subset s e^{*}(3),
$$

the $6-l$ constrained twists can be obtained since they are the twists that cause no power transfer in the direction of the free wrench, i.e.:

$$
{ }^{i} W_{\mathrm{F}}^{j} T_{\mathrm{C}}=0 \forall i, j .
$$

The algebras are now:

$$
\begin{aligned}
s e(3) & =\mathscr{T}_{\mathrm{F}} \oplus \mathscr{T}_{\mathrm{C}} \\
s e^{*}(3) & =\mathscr{W}_{\mathrm{F}} \oplus \mathscr{W}_{\mathrm{C}} .
\end{aligned}
$$

\section{Compliant kinematic coupling}

A kinematic pair applies an action and, equal but opposite, reaction force to connected bodies, and together with Equations (7.8) and (7.9), they describe fundamental properties of the kinematic pair. However, these properties do not hold when a compliant actuator, as opposed to a stiff actuator, is used. Due to the internal (rotor and spring) dynamics in a compliant actuator, the forces associated to the stator (controlled input force) and the output (elastic force) are different, which is not possible to implement in a single kinematic pair. This means that to incorporate the compliant actuator as shown in Figure 7.3, two kinematic pairs for each coupling between bodies are needed, as shown in Figure 7.7a, i.e., $\mathrm{KP}_{a}$ to describe the controlled input side (side $a$ ), and $\mathrm{KP}_{b}$ to describe the compliant side (side $b$ ). To $\mathrm{KP}_{a}$, the input force $u_{p}$, generated in the VSA motor, is associated that is applied to both the left rigid body as well as to the internal rotor inertia $m$. To $\mathrm{KP}_{b}$, the adjustable elastic element is associated, that delivers a force which is applied to both the right rigid body as well as to the internal rotor inertia $m$. In this way, the fundamental properties of a kinematic pair are still satisfied. Here, the compliant kinematic coupling is defined in such a way that the onedimensional VSA model of Section 7.2.1 can be incorporated directly, as shown in Figure 7.7b. In that way, the two distinct interaction forces of the VSA as a consequence of the internal 


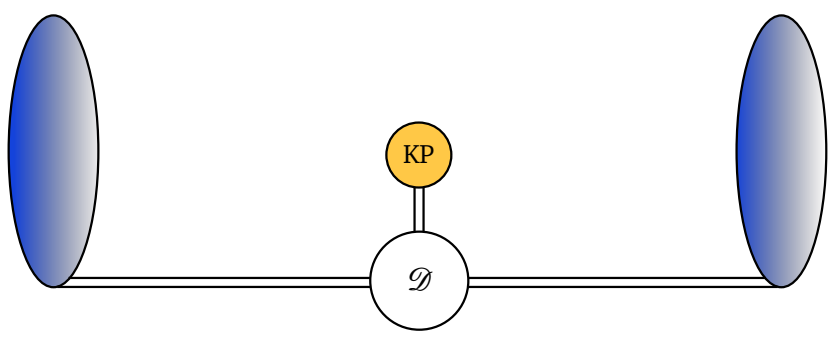

(a) A one-port kinematic pair.

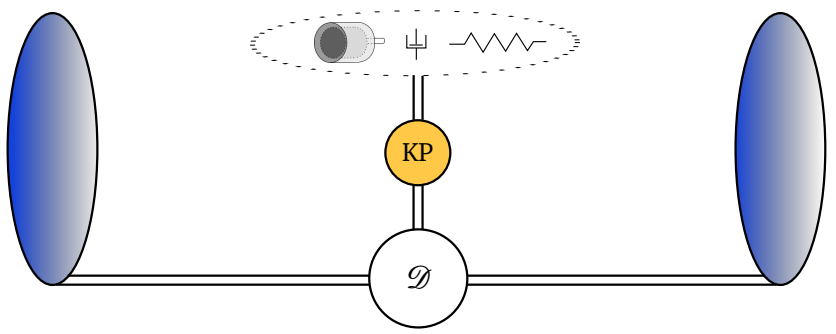

(b) A two-port kinematic pair, with an additional power supplying element like a motor, a damper, or a spring.

Figure 7.6: A one-port (Figure 7.6a) and two-port (Figure 7.6b) kinematic pair, constraining the relative motion between two bodies. The additional nodic power supplying element applies a force on both bodies (opposite, and of equal magnitude), as action and reaction. The Dirac structure $\mathscr{D}$ divides the power flow between the connected ports.

dynamics, $f_{b}^{a}:=f_{\text {intv }_{2}}$ and $f_{b}^{b}:=f_{\text {intv }_{3}}$ as given in Equation (7.7), are transformed into two distinct wrenches, applied on the separate connected bodies, through $\mathrm{KP}_{a}$ and $\mathrm{KP}_{b}$.

In the remainder of this chapter, a choice is made for the representation of this kinematic coupling. The coordinate basis is chosen as shown in Figure 7.7b, with body fixed frames $\left(\Psi_{a}\right.$ and $\Psi_{b}$ ) lying on the screw motion axis. Even if there is no intrinsic metric in se(3) defining orthogonality, an "orthonormal" basis is defined by choosing the canonical basis twists $e_{i}$ as:

$$
\begin{aligned}
e_{1} & =\left[\begin{array}{llllll}
1 & 0 & 0 & 0 & 0 & 0
\end{array}\right]^{\mathrm{T}} \\
e_{2} & =\left[\begin{array}{llllll}
0 & 1 & 0 & 0 & 0 & 0
\end{array}\right]^{\mathrm{T}} \\
e_{3} & =\left[\begin{array}{llllll}
0 & 0 & 1 & 0 & 0 & 0
\end{array}\right]^{\mathrm{T}} \\
e_{4} & =\left[\begin{array}{llllll}
0 & 0 & 0 & 1 & 0 & 0
\end{array}\right]^{\mathrm{T}} \\
e_{5} & =\left[\begin{array}{llllll}
0 & 0 & 0 & 0 & 1 & 0
\end{array}\right]^{\mathrm{T}} \\
e_{6} & =\left[\begin{array}{llllll}
0 & 0 & 0 & 0 & 0 & 1
\end{array}\right]^{\mathrm{T}} .
\end{aligned}
$$

Every twist is a linear combination of these basis twists, i.e., $\operatorname{span}\left\{e_{1}, \ldots, e_{6}\right\}=\operatorname{se}(3)$. A basis for the wrenches is then given by:

$$
e^{j}:\left\langle e^{j}, e_{i}\right\rangle=0 \forall i \neq j
$$

and, therefore, $\operatorname{span}\left\{e^{1}, \ldots, e^{6}\right\}=s e^{*}(3)$. Because of the choice of this basis, the constrained twist or wrench of a kinematic pair can directly be defined when a free twist or wrench is known or defined.

The free wrenches of both kinematic pairs (pair $a$ and pair $b$ in Figure 7.7b) can be given 


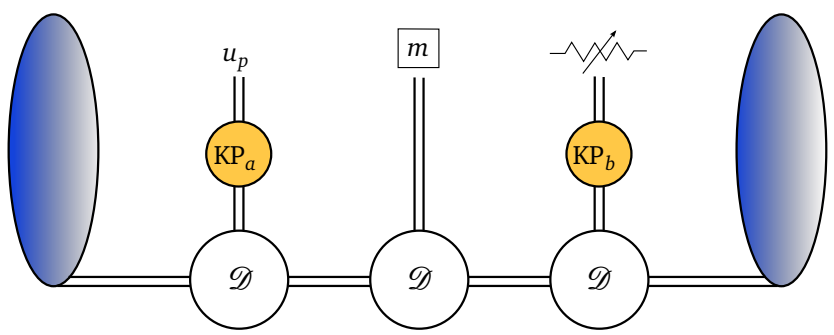

(a) Two two-port kinematic pairs $\left(K P_{a}\right.$ and $\left.K P_{b}\right)$ with power supplying elements that are associated to the corresponding VSA elements. Inertia $m$ is associated to the rotor inertia.

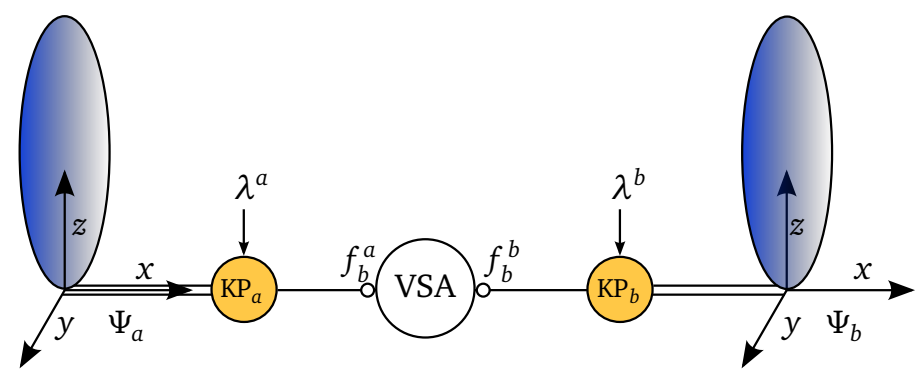

(b) Proposed implementation of the compliant kinematic coupling through a onedimensional VSA.

Figure 7.7: Compliant coupling between bodies using two separate kinematic pairs. Figure 7.7a associates separate power supplying elements to the kinematic pairs, while the proposed implementation of Figure 7.7b allows the direct connection of the one-dimensional VSA.

by:

$$
\left[\begin{array}{c}
{ }^{1} W_{\mathrm{F}}^{a, a} \\
\vdots \\
{ }^{l} W_{\mathrm{F}}^{a, a}
\end{array}\right]:=W_{\mathrm{F}}^{a, a},\left[\begin{array}{c}
{ }^{1} W_{\mathrm{F}}^{b, b} \\
\vdots \\
{ }^{l} W_{\mathrm{F}}^{b, b}
\end{array}\right]:=W_{\mathrm{F}}^{b, b},
$$

where ${ }^{n} W_{\mathrm{F}}^{i, j}$ indicates the $n$-th free wrench on body fixed frame $\Psi_{j}$ expressed with coordinates in frame $\Psi_{i}$. These free wrenches are the wrenches on a body as a result of the actuator forces $f_{b} \in \mathbb{R}^{l}$, i.e., the forces of the boundary vertices transformed to body wrenches by the kinematic pairs. The constraint wrenches are then given by:

$$
\left[\begin{array}{c}
{ }^{1} W_{\mathrm{C}}^{a, a} \\
\vdots \\
{ }^{6-l} W_{\mathrm{C}}^{a, a}
\end{array}\right]:=W_{\mathrm{C}}^{a, a}, \quad\left[\begin{array}{c}
{ }^{1} W_{\mathrm{C}}^{b, b} \\
\vdots \\
{ }^{6-l} W_{\mathrm{C}}^{b, b}
\end{array}\right]:=W_{\mathrm{C}}^{b, b} .
$$

The joint constraint forces $\lambda \in \mathbb{R}^{6-l}$, which are Lagrange multipliers that keep the relative velocity between two connected bodies in the constraint directions to zero, are transformed to these constraint wrenches. The kinematic pair transformations are then:

$$
J_{k p}^{a}:=\left[\frac{W_{\mathrm{F}}^{a, a}}{W_{\mathrm{C}}^{a, a}}\right] \quad J_{k p}^{b}:=\left[\frac{W_{\mathrm{F}}^{b, b}}{W_{\mathrm{C}}^{b, b}}\right] .
$$

Due to the choice of basis, and specifically the alignment of the local $x$-axes of the frames $\Psi_{a}$ and $\Psi_{b}$, it holds that $W_{\mathrm{F}}^{a, a}=W_{\mathrm{F}}^{b, b}$ in (7.10), $W_{\mathrm{C}}^{a, a}=W_{\mathrm{C}}^{b, b}$ in (7.11), and $J_{k p}^{a}=J_{k p}^{b}$ in (7.12). 
These equalities hold in general if the wrenches of both sides are expressed in one of the two frames. For an $l$ degree of freedom kinematic pair, the wrenches expressed in $\Psi_{a}$ become:

$$
\left[\begin{array}{c}
W_{k p}^{a, a \mathrm{~T}} \\
W_{k p}^{a, b} \mathrm{~T}
\end{array}\right]=\left[\begin{array}{cc}
J_{k p}^{\mathrm{T}} & 0 \\
0 & J_{k p}^{\mathrm{T}}
\end{array}\right]\left[\begin{array}{c}
f_{b}^{a} \\
\lambda \\
f_{b}^{b} \\
-\lambda
\end{array}\right],
$$

where $f_{b}^{a}, f_{b}^{b} \in \mathbb{R}^{l}$ and $\lambda \in \mathbb{R}^{6-l}$. The Lagrange multipliers have a positive and negative sign, because they act as action and reaction on either one of the bodies.

Remark. Note that in the implementation of Figure 7.7b, the rotor inertia degenerates to a onedimensional inertia that acts only in the direction of the VSA's rotation. Of course, the VSA may be attached to a rigid body and undergo spatial motion, which would require a six-dimensional inertia to properly represent the kinetic energy, as is shown in Figure 7.7a. However, it depends on the specific realization of the mechanism whether that inertia indeed should to be taken into account. If the VSA is located on a body, it should be taken into account if the rotor inertia is non negligible with respect to the bodies. If the VSA is, for instance, attached to a fixed base and tendons are used to transmit the power, the inertia does not have to be taken into account, since its kinetic energy will always be zero. In the former case, the part of the six-dimensional rotor inertia that is not associated to the one-dimensional rotation of the VSA can be taken into account by properly changing coordinates and adding the inertia to the body with respect to which the rotor inertia has constraints that are kept by the Lagrange multipliers.

\subsubsection{Rigid bodies}

The rigid bodies connect to the kinematic coupling such that their relative motion is constrained. The port-Hamiltonian equations of a rigid body, as can be found in [107] and [109], were used in this model, i.e.:

$$
\begin{aligned}
{\left[\begin{array}{c}
\dot{\phi}_{i}^{0} \\
\left(\dot{P}_{i}^{i}\right)^{\mathrm{T}}
\end{array}\right] } & =\left[\begin{array}{cc}
0 & \kappa\left(\phi_{i}^{0}\right) \circ A d_{H_{i}^{0}} \\
-A d_{H_{i}^{0}}^{\mathrm{T}} \circ \kappa^{\mathrm{T}}\left(\phi_{i}^{0}\right) & -\tilde{P}_{i}^{i} \wedge
\end{array}\right]\left[\begin{array}{c}
\frac{\partial H}{\partial \phi_{i}^{0}} \\
\frac{\partial H}{\partial P_{i}^{i}}
\end{array}\right] \\
& +\left[\begin{array}{c}
0 \\
A d_{H_{i}^{0}}^{\mathrm{T}}
\end{array}\right] W_{\mathrm{con}}^{0, i \mathrm{~T}}+\left[\begin{array}{c}
0 \\
A d_{H_{i}^{0}}^{\mathrm{T}}
\end{array}\right] W_{\mathrm{int}}^{0, i^{\mathrm{T}}} \\
T_{\operatorname{con}_{i}{ }^{0,0}} & =\left[\begin{array}{ll}
0 & A d_{H_{i}^{0}}
\end{array}\right]\left[\begin{array}{c}
\frac{\partial H}{\partial \phi_{i}^{0}} \\
\frac{\partial H}{\partial P_{i}^{i}}
\end{array}\right] \\
T_{\text {int }_{i}{ }^{0,0}} & =\left[\begin{array}{lll}
0 & A d_{H_{i}^{0}}
\end{array}\right]\left[\begin{array}{c}
\frac{\partial H}{\partial \phi_{i}^{0}} \\
\frac{\partial H}{\partial P_{i}^{i}}
\end{array}\right]
\end{aligned}
$$

where $\phi_{i}^{0}$ is the finite twist, or the exponential coordinate of configuration $H_{i}^{0}$ (as opposed to the infinitesimal or instantaneous twist $T_{\bullet}^{\bullet \bullet \bullet}$ ), which is related to the homogeneous configuration by the exponential map $H_{i}^{0}=e^{\tilde{\phi}_{i}^{0}} \cdot{ }^{2} P_{i}^{i}$ is the rigid body momentum of body $i$ expressed in

\footnotetext{
${ }^{2}$ The finite twist can be thought of as a configuration change resulting from applying an instantaneous twist for one second. Therefore, the finite twist can be thought of as a configuration itself. Note that the finite twist is not a globally continuous parametrization of the special Euclidean group $S E(3)$ and it has singularities at multiples of $2 \pi$ (at which rotations are the same).
} 
body frame $\Psi_{i}, \kappa(\bullet)$ is the map from instantaneous twist to finite twist, $A d_{H_{i}^{0}}$ is the coordinate change from body frame $\Psi_{i}$ to the inertial frame $\Psi_{0}$, and $\tilde{P}_{i}^{i} \wedge$ is the map from instantaneous twist to rate of change of momentum. The partial derivatives of the Hamiltonian $H$, storing kinetic and potential energy, are the efforts and $T_{i}^{0,0}$ is the instantaneous twist of body $i$ with respect to the inertial frame $\Psi_{0}$, expressed in the inertial frame $\Psi_{0}$. Subscript 'con' indicates a correspondence to the control port, while subscript 'int' indicates a correspondence to the interaction port.

\subsubsection{Overall interconnection on graphs}

The combination of the components treated in the previous sections synthesizes a compliant manipulator: rigid bodies are kinematically coupled such that their relative motion is constrained and compliant actuators connect to kinematic pairs within this kinematic coupling to deliver power. In this section, the modeling of compliant mechanisms on graphs is elaborated.

\section{Kinematic structure}

A manipulator segment without actuators is shown in Figure 7.8a of which the kinematic structure graph (or primary graph) is shown in Figure 7.8b. It defines the interconnection of the rigid bodies (vertices) by the kinematic coupling (edges), i.e., it defines the kinematic structure of a serial chain manipulator. VSAs can interact with the kinematic pairs and, thereby, with the rigid bodies, by introducing boundary vertices inside the kinematic coupling edges, as shown in Figure 7.8c. These vertices are interfaces for the connection of VSAs, and are massless. The kinematic pair wrenches, of which there are two per kinematic coupling, as elaborated in Section 7.2.2, are the wrenches that act on the rigid bodies. This is captured by the internal vertex incidence matrix $B_{\mathfrak{G} \mathrm{kp}_{i}}$ of the graph in Figure 7.8c, which maps kinematic pair edge flows $f_{\mathrm{kp}}$ to rigid body vertex flows $f_{\mathrm{RB}}$, i.e.:

$$
f_{\mathrm{RB}}=B_{\mathfrak{G} \mathrm{kp}_{i}} f_{\mathrm{kp}} \text {. }
$$

The vertex flows $f_{\mathrm{RB}}$ are the rigid body wrenches $W_{\mathrm{RB}}^{\mathrm{T}}=\left[\begin{array}{llll}W_{\mathrm{RB}_{0}} & \ldots & W_{\mathrm{RB}_{\mathrm{n}}}\end{array}\right]^{\mathrm{T}}$ (in which $W_{\mathrm{RB}_{\mathrm{i}}}=W_{\text {con }}^{0, i \mathrm{~T}}$ as given in (7.14)) and the edge flows $f_{\mathrm{kp}}$ are the kinematic pair wrenches $W_{\mathrm{kp}}^{\mathrm{T}}=\left[\begin{array}{lll}W_{\mathrm{kp}_{1}}^{a} W_{\mathrm{kp}_{1}}^{b} & \ldots & W_{\mathrm{kp}_{n}}^{a} W_{\mathrm{kp}_{n}}^{b}\end{array}\right]^{\mathrm{T}}$ (where $W_{\mathrm{kp}_{i}}^{a} W_{\mathrm{kp}_{i}}^{b}$ are as was given in (7.13)). The kinematic pairs can have $l$ degrees of freedom, which is not represented in the graph of Figure 7.8c. Moreover, because the boundary vertices are massless, a map from the kinematic pair flows $f_{\mathrm{kp}}$ to forces in the boundary vertices $f_{b}$ is not possible (a massless object cannot have momentum and, therefore, cannot be subject to a force). However, for a one degree of freedom kinematic pair, a dual incidence map, as given in (7.4), holds (a massless object can have a velocity, and be subject to it), i.e.:

$$
e_{b}=B_{\mathfrak{G} \mathrm{kp}_{b}} e_{\mathrm{kp}}
$$

where $e_{b}$ are the boundary vertex efforts, i.e., the velocities of the boundary vertices, $B_{\mathfrak{G} \mathrm{kp} b}$ is the boundary vertex incidence matrix, and $e_{\mathrm{kp}}$ are the kinematic pair edge efforts, i.e., the twists $T_{\mathrm{kp}}$ that are conjugated with kinematic pair wrenches $W_{\mathrm{kp}}$. A serial kinematic structure, where the connection is determined by the graph, is now obtained and defined, allowing the connection of compliant actuators. 


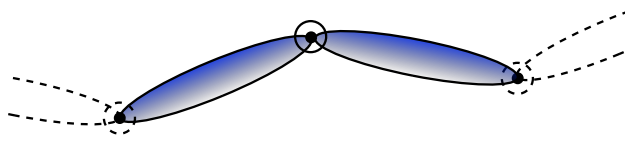

(a) Manipulator segment.

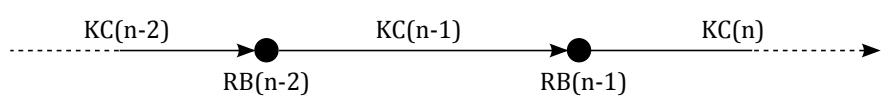

(b) Partial (non-interacting) kinematic structure graph (or primary graph).

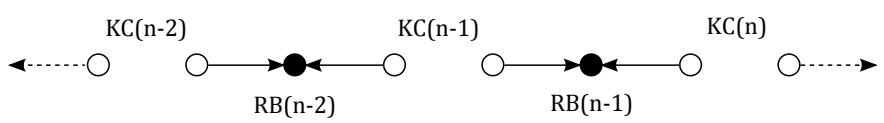

(c) Partial kinematic structure graph, where the kinematic pairs (two per kinematic coupling) can interact with another system through boundary vertices (open circles).

Figure 7.8: Manipulator segment with the corresponding kinematic structure (or primary) graph. $R B(n)$ is the $n$-th rigid body along the mechanism and $K C(n)$ indicates the $n$-th kinematic coupling between two rigid bodies, consisting of two kinematic pairs each.

\section{Actuators connection topology}

The interacting kinematic coupling allows the connection of power supplying elements, which are in general actuators. The connection of actuators to the system is traditionally done in a uniarticulation way, where every actuator delivers power to only one joint. This modeling framework based on graphs, however, allows the straightforward inclusion of biarticulation, or even multiarticulation, actuators, delivering power to multiple consecutive joints, in the kinematic structure $[96,97]$. This is done by defining an actuators morphology, as a graph that defines the connection between $m$ actuators, and $n$ kinematic couplings with $l$ degrees of freedom (making $L$ degrees of freedom in total). Figure 7.9a shows the connection of various VSAs to the kinematic pairs that were introduced in Figure $7.8 \mathrm{c}$, together with the actuators connection graph in Figure 7.9b. Note that the kinematic coupling to which VSA $(m-1)$ and $\operatorname{VSA}(m)$ are connected, is a two degrees of freedom coupling, which is defined in this graph by separate boundary vertex pairs for each degree of freedom. The $\operatorname{VSA}(m-1)$ connects to the second degree of freedom in a uniarticulation way, while $\operatorname{VSA}(m)$ connects in a biarticulation way to the first degree of freedom, as well as to the right-most one degree of freedom coupling.

This actuators connection topology is defined by a mapping where the VSA edges map to the boundary vertices of the kinematic pairs using the incidence mappings $B_{\mathfrak{G} \text { act } b}$ and $B_{\mathfrak{G} \text { act } b}^{\mathfrak{g}}$ which are defined by the graph in Figure 7.9. Note that this mapping is partially gyrating, since the forces to be mapped to the boundary vertices are both a flow and an effort. Therefore, the mapping becomes:

$$
f_{b}=\left[\begin{array}{ll}
B_{\mathfrak{G} \text { act } b} & B_{\mathfrak{G} \text { act } b}^{\mathfrak{g}}
\end{array}\right]\left[\begin{array}{l}
f_{\text {act } u} \\
e_{\text {act } k}
\end{array}\right]
$$

where $f_{b}=\left[\begin{array}{lll}f_{b_{1}}^{a} f_{b_{1}}^{b} & \ldots & f_{b_{L}}^{a} f_{b_{L}}^{b}\end{array}\right]^{\mathrm{T}} \in \mathbb{R}^{L}$ are the kinematic pair's (sides $a$ and $b$ ) boundary vertex flows, $f_{\text {act } u} \in \mathbb{R}^{m}$ are the actuators input force flows $u_{p}$ from (7.7), and $e_{\text {act } k} \in \mathbb{R}^{m}$ are the actuators spring force efforts $\frac{\partial H}{\partial s}$ from (7.7), needing the gyrating mapping $B_{\mathfrak{G} \text { act } b}^{\mathfrak{g}}$, corresponding to $B_{\mathfrak{G} k b}^{\mathfrak{g}}$ as given in (7.6). Likewise, these actuator forces also map to their internal 


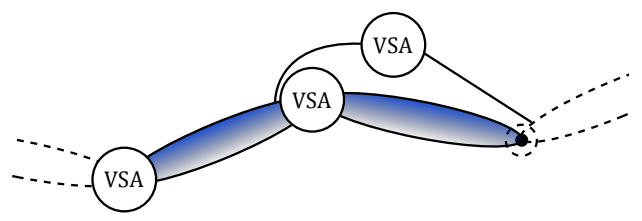

(a) Manipulator segment.

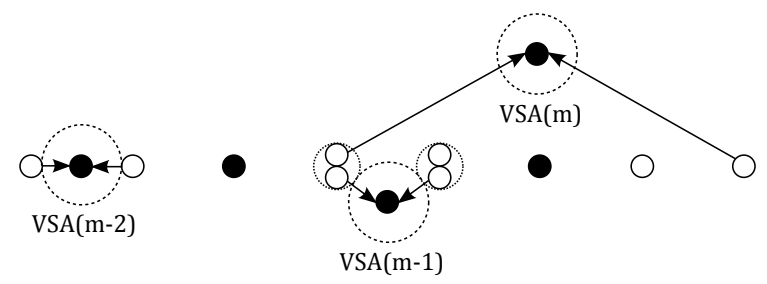

(b) Partial actuators connection graph.

Figure 7.9: Example of uni- and bi-articulation actuators connection to the manipulator segment.

vertex, the rotor, in a gyrating and non-gyrating way using $B_{\mathfrak{G} \text { act } i}$ and $B_{\mathfrak{G} \text { acti }}^{\mathfrak{g}}$, according to:

$$
f_{p}=\left[\begin{array}{ll}
B_{\mathfrak{G} \text { act } i} & B_{\mathfrak{G} \text { act } i}^{\mathfrak{g}}
\end{array}\right]\left[\begin{array}{l}
f_{\text {act } u} \\
e_{\text {act } k}
\end{array}\right],
$$

where $f_{p}=\left[\begin{array}{lll}f_{p_{1}} & \ldots & f_{p_{m}}\end{array}\right]^{\mathrm{T}} \in \mathbb{R}^{m}$ are the flows of the internal (rotor mass) vertex.

The mapping $\left[\begin{array}{ll}B_{\mathfrak{G} \text { act } b} & B_{\mathfrak{G} \text { act } b}^{\mathfrak{g}}\end{array}\right]$ can be simply adjusted according to the changes made to the actuators connection graph of Figure $7.9 \mathrm{~b}$ to change the actuators connection. This is possible without any further adjustments of the kinematic structure graph defining all kinematic couplings.

\subsection{Case Study: Comparison to Traditional Modeling}

This section shows the benefits of using a modular model based on port-Hamiltonian systems and graphs, that is presented in this chapter, as opposed to the more traditional EulerLagrange model. In particular, it will be made explicit that the model proposed here is much more flexible regarding changes in actuator morphology and can be a tool in designing optimal complex compliant manipulators.

The model of a two-body (three including the base), three degrees of freedom manipulator with compliant actuators, as shown in Figure 7.10, is derived. The first joint is a one degree of freedom joint allowing rotation around the z-axis, while the second joint is a two degrees of freedom joint allowing in plane as well as out of plane motion. Firstly, a model is considered in the situation where all three joints (generalized coordinates $q_{1}, q_{2}$ and $q_{3}$ ) are actuated by a uniarticulation VSA (VSA1, VSA2 and VSA3, respectively). Secondly, a model is considered where VSA2 is attached in a biarticulated way on the first degree of freedom of the second joint, hence spanning both $q_{1}$ and $q_{2}$. In both situations, a frictionless system with gravity is considered, with linear elastic behavior (the elastic force is $\tau_{E i}=k_{i} l(q)$ and where $k_{1}=$ $k_{2}=10 \mathrm{Nm} / \mathrm{rad}$ ). A constant torque of $1 \mathrm{Nm}$ is applied by VSA2 and VSA3, while VSA1 is left unpowered. No external disturbance forces are present. It is assumed that a VSA's rotor inertia is negligible with respect to the body to which it is attached, except for the rotor inertia acting in the VSA's rotating direction. Moreover, there can be an inertial coupling, i.e., the 


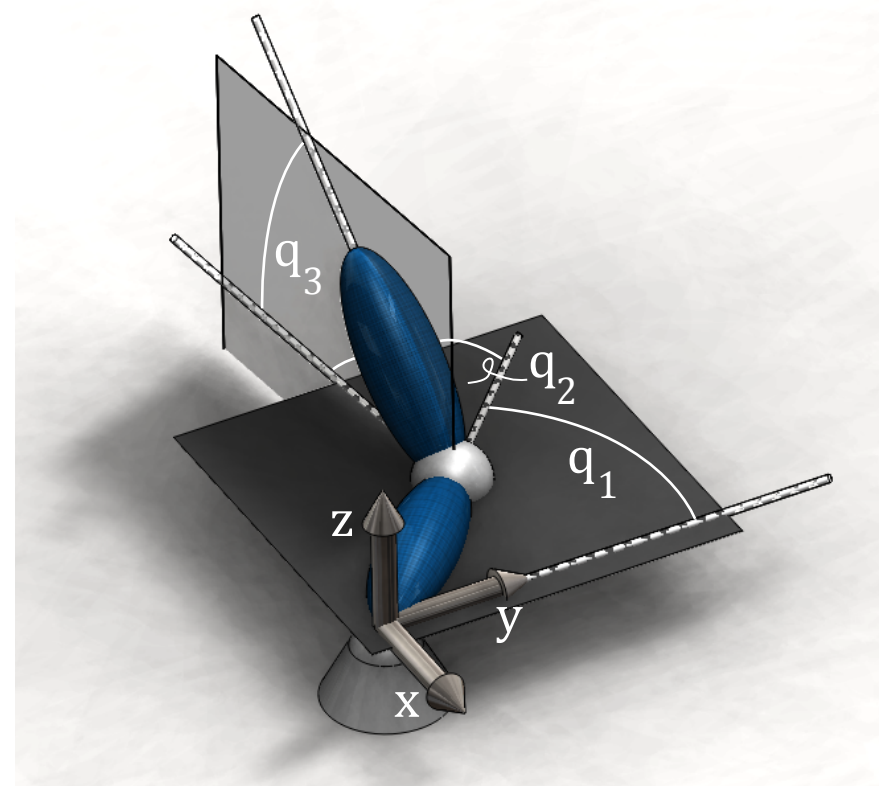

Figure 7.10: Kinematic structure of a three degrees of freedom manipulator, simulated twice with different actuator topologies: once uniarticulated and once biarticulated.

rotation of the rotors is not only due to their self rotation, but also due to the rotation of the links.

\subsubsection{Uniarticulated manipulator}

\section{Euler-Lagrange model}

A generic and well-known Euler-Lagrange model of a compliant manipulator [93] can be given by:

$$
\begin{gathered}
{\left[\begin{array}{cc}
M_{m} & S^{\mathrm{T}}(q) \\
S(q) & M(q)
\end{array}\right]\left[\begin{array}{c}
\ddot{\theta} \\
\ddot{\bar{q}}
\end{array}\right]+\left[\begin{array}{cc}
0 & C_{\theta}(q, \dot{q}) \\
C_{q}(q, \dot{q}) & C(q, \dot{q})
\end{array}\right]\left[\begin{array}{c}
\dot{\theta} \\
\dot{\bar{q}}
\end{array}\right]} \\
+\left[\begin{array}{cc}
D_{m} & 0 \\
0 & D
\end{array}\right]\left[\begin{array}{c}
\dot{\theta} \\
\dot{\bar{q}}
\end{array}\right]+\left[\begin{array}{c}
0 \\
g(q)
\end{array}\right]+\left[\begin{array}{c}
\tau_{E 1} \\
\tau_{E 2}
\end{array}\right] \\
=\left[\begin{array}{c}
\tau_{u} \\
\tau_{\text {ext }}
\end{array}\right],
\end{gathered}
$$

where $\theta$ are the generalized rotor coordinates, $\bar{q}$ are the generalized link coordinates and where $q=\left[\begin{array}{cc}\theta & \bar{q}\end{array}\right]^{\mathrm{T}}$. The motor equations and the link equations are in general dynamically coupled through the elastic torques $\tau_{E i}$ as well as through the inertial coupling term $S(q)$. These equations can be found by mapping the inertia tensor and stiffness (using the potential elastic energy) in world coordinates to generalized coordinates, through the Jacobians of the forward kinematics maps, and calculating the Christoffel's symbols to find the Coriolis and centrifugal term. It is assumed that the stiffness can be directly changed by a control input $\tau_{k}$, in accordance with the port-Hamiltonian model of the VSA in Section 7.2.1, i.e., $\dot{k}=\tau_{k}$. 
Due to the assumptions, it holds that $D_{m}=D=0, g(q)=\left[\begin{array}{ccc}0 & 0 & -9.81 \cos \left(\overline{q_{3}}\right)\end{array}\right]$, $\tau_{u}=\left[\begin{array}{lll}0 & 1 & 1\end{array}\right]^{\mathrm{T}}, \tau_{\mathrm{ext}}=\left[\begin{array}{ccc}0 & 0 & 0\end{array}\right]^{\mathrm{T}}$, and $\tau_{k}=\left[\begin{array}{ccc}0 & 0 & 0\end{array}\right]^{\mathrm{T}}$.

\section{Graph model}

The model elaborated in Section 7.2 is used. The kinematic structure of the manipulator is given by the graph in Figure 7.11a.
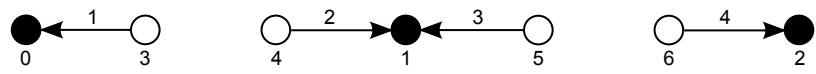

(a) Kinematic structure graph.
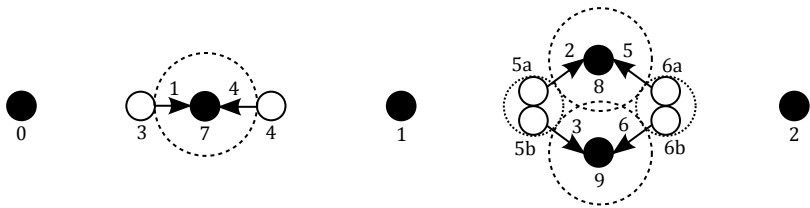

(b) Actuators connection graph, defining the interconnection of the actuators to the kinematic pairs.

Figure 7.11: Graphs of the manipulator shown in Figure 7.10, where uniarticulation is applied.

From Figure 7.11a, the internal vertex incidence matrix $B_{\mathfrak{G ~ k p}_{i}}$, mapping flows as in (7.15), is found:

$$
B_{\mathfrak{G} \mathrm{kp}_{i}}=\left[\begin{array}{cccc}
1 & 0 & 0 & 0 \\
0 & 1 & 1 & 0 \\
0 & 0 & 0 & 1
\end{array}\right] .
$$

The graph in Figure 7.11b defines the interconnection of the actuators to the kinematic pairs, which is defined by the boundary vertex incidence matrix $B_{\mathfrak{G} \text { act } b}=\left[\begin{array}{ll}B_{\mathfrak{G} \text { act } b} & B_{\mathfrak{G} \text { act } b}^{\mathfrak{g}}\end{array}\right]$, mapping flows and efforts as in (7.16):

$$
B_{\mathfrak{G} \text { act } b}=\left[\begin{array}{cccccc}
-1 & 0 & 0 & 0 & 0 & 0 \\
0 & 0 & 0 & -1 & 0 & 0 \\
0 & -1 & 0 & 0 & 0 & 0 \\
0 & 0 & 0 & 0 & -1 & 0 \\
0 & 0 & -1 & 0 & 0 & 0 \\
0 & 0 & 0 & 0 & 0 & -1
\end{array}\right] .
$$

This completely defines the dynamics of the system.

Both identical simulations can be seen in Figure 7.13.

\subsubsection{Biarticulated manipulator}

\section{Euler-Lagrange model}

The equations in (7.17) are still valid, but since the second uniarticulation actuator has changed to a biarticulation actuator, the map from actuator world coordinates to generalized coordinates has changed. This means that the inertia tensor in generalized coordinates will change, the Christoffel symbols will change as a result of that, and the stiffness in generalized coordinates will change as well. Hence, the complete equations of motion are different and have to be recalculated as a consequence of the actuator relocation. 


\section{Graph model}

The graph in Figure 7.11a defines the topology of the biarticulated manipulator; the kinematic structure has not changed and, hence, that graph is still valid for this model.

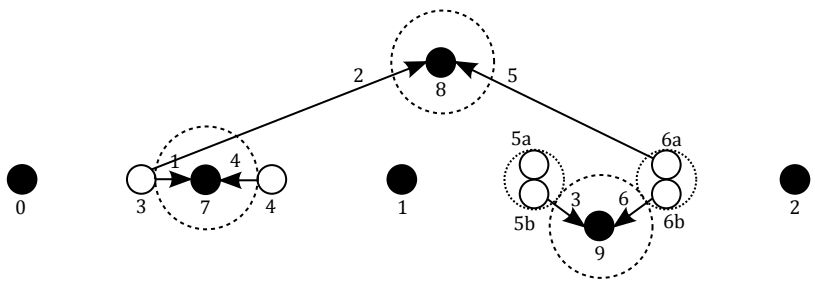

Figure 7.12: Graph of the biarticulated manipulator in Figure 7.10.

Therefore, this biarticulation model only requires a change in the incidence matrix $B_{\mathfrak{G} \text { act } b}$, obtained from the graph:

$$
B_{\mathfrak{G} \text { act } b}=\left[\begin{array}{cccccc}
-1 & -1 & 0 & 0 & 0 & 0 \\
0 & 0 & 0 & -1 & 0 & 0 \\
0 & 0 & 0 & 0 & 0 & 0 \\
0 & 0 & 0 & 0 & -1 & 0 \\
0 & 0 & -1 & 0 & 0 & 0 \\
0 & 0 & 0 & 0 & 0 & -1
\end{array}\right] .
$$

Note that only a change of two numbers (in bold) is required to define the biarticulation model.

Both identical simulations, albeit obviously different from the uniarticulation model, can also be seen in Figure 7.13.

\subsubsection{Simulation results}

The simulations shown here validate the proposed model, since this graph model behavior and the well established Euler-Lagrange model behavior are identical in both situations. It has been made explicit that the changes required in the graph model are very basic and much less severe than the changes that have to be made to the Euler-Lagrange model. Although the example treats three degrees of freedom, it really shows the potential of this model for larger and more complex systems. In general, when considering uni/bi/tri/... articulation, actuators can be connected in $N=\frac{1}{2} n(n+1)$ different locations, where $n$ is the number of degrees of freedom. When considering, for instance, anthropomorphic manipulators where $n=7$, it will result in $N=28$ locations. Then, a model that easily allows the change of a system's topology can aid in making design decisions.

\subsection{Discussion}

Any manipulator with an arbitrary amount of compliant actuators connected to arbitrary joints can be easily modeled by adjusting incidence matrices. This is because the kinematic structure of the system is described independently from the actuators connection topology, facilitated by the usage of the port-Hamiltonian framework on graphs. Various actuator connection topologies are possible on the same kinematic structure, which makes the ease of this modeling 
approach explicit. Although this chapter only focused on serial chain manipulators, in fact any manipulator (serial, fork, or even parallel) can be modeled using this method.

It has been shown that the proposed model can be easily adapted to different actuator connection topologies by changing only two elements in the incidence matrix, which is a result of the port-based modeling and graph theoretic topology description which decouples a system's morphology from its dynamics. This is in contrast with the more fundamental adaptation needed in the Euler-Lagrange model. Surely, automation and software tools reduce the effort of changing a model to suit a specific situation, but nonetheless does this model have the potential to provide new possibilities for designing manipulators and their actuator placement and configuration. This ease of adaptation also holds for changing a kinematic coupling, i.e., changing the free relative motion of the connection of two bodies is simply done by adapting (7.10).

\subsection{Conclusion}

This chapter proposed a model based on graph theory and the port-Hamiltonian framework for generic compliant manipulators. Previous work, which showed the modeling of a variable stiffness actuator on a graph, has been extended and a kinematic coupling that allows the connection of compliant actuators was introduced. It was shown that a manipulator's topology is decoupled from its dynamics, which allows a straightforward change of actuators' connection by changing elements in an incidence matrix. An example, where a uni- and biarticulated two degrees of freedom compliant manipulator were modeled using the proposed model and the traditional Euler-Lagrange model, made this ease of adaptation explicit. 

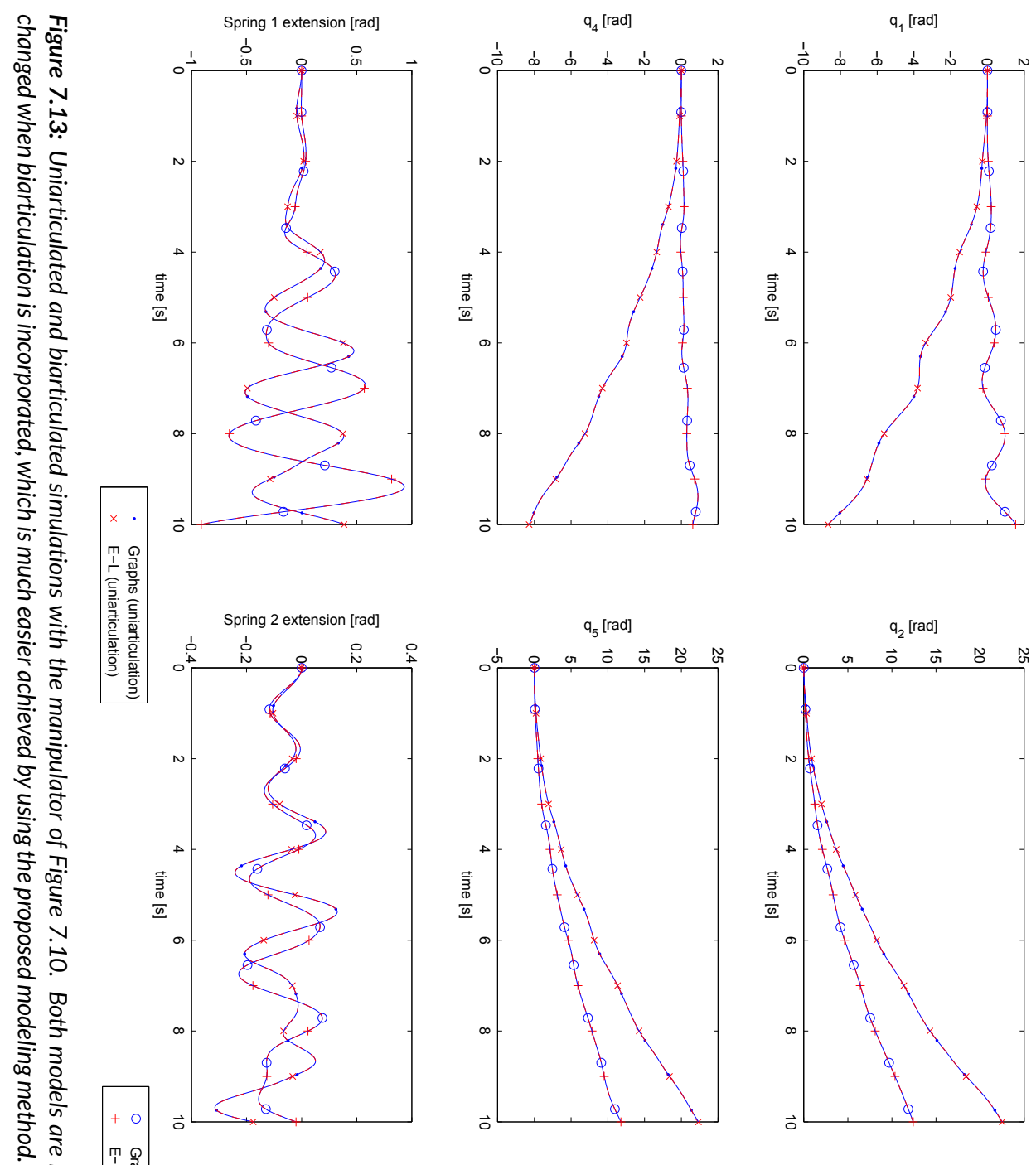

용
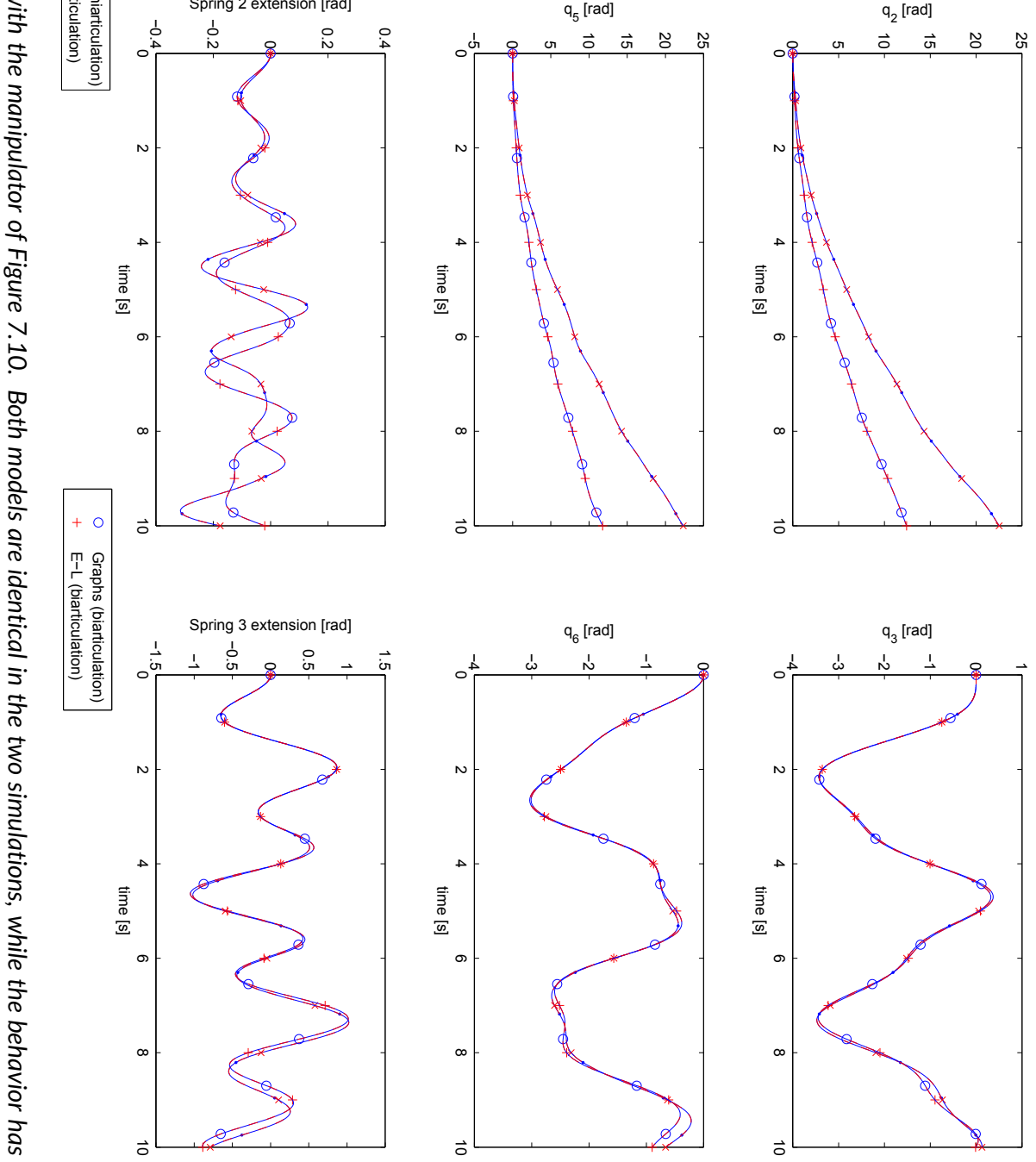


\section{CHAPTER 8}

\section{Manipulator Stiffness Modeling, Design, and Control}

This chapter proposes a description of the stiffness and compliance of compliant robotic manipulators that are modeled using graphs, and the stiffness control using a geometry based metric. The graph model is used to obtain the actuation Jacobian in a structured way, which is used to transform stiffnesses and compliances between actuation space and configuration space. The modeling of end effector compliance as a result of an actuation stiffness is experimentally verified on a two degrees of freedom planar manipulator driven by variable stiffness actuators. Furthermore, a metric is proposed to control the stiffnesses of these actuators such that the end effector stiffness approximates a desired end effector stiffness. This metric compares the stiffnesses in joint space, and returns the optimal actuator connection topology and individual actuator stiffnesses to approximate the desired stiffness. A simulation with a two degrees of freedom compliant planar manipulator is done, which shows the validity of the metric.

This chapter has been submitted for possible publication as:

[110] S. S. Groothuis, S. Stramigioli, and R. Carloni, "Stiffness analysis and control of compliant robotic manipulators," IEEE Transactions on Robotics, 2016. Submitted 


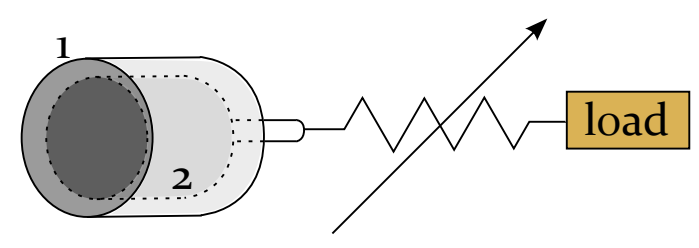

Figure 8.1: Schematic view of a variable stiffness actuator, showing a motor with stator and rotor, an adjustable elastic element, and an attached load.

$\mathrm{R}^{\mathrm{o}}$ овотіс systems are more and more finding their way in human daily life, through care, assistive, and leisure applications. If those systems are to be successful in human daily life, their safety with regard to humans is of utmost importance. In particular larger systems with manipulating capabilities should be subject to severe safety conditions, since human injury may occur [4]. Therefore, various safety measures have been implemented to ensure safe behavior, among which is (joint) compliance. A manipulator's joint compliance can be achieved by using variable stiffness actuators. Particularly, variable series elastic actuators are often chosen, like the one shown in Figure 8.1, showing a motor with stator and rotor, an adjustable elastic element, and an attached load. These joint compliances cause the end effector of the manipulator, or any other point under consideration, to have compliance in work space, which can geometrically be visualized by an ellipsoid centered at the end effector, and scaled and rotated according to the compliance settings and manipulator configuration. Furthermore, the articulation type and distribution also influence this property. An articulation type can best be explained by referring to the human arm, and specifically to the biceps and triceps. These muscles are biarticulation muscles, since they span two joints, namely the shoulder, and the elbow. This is in contrast with muscles that span only one joint, and are, therefore, called uniarticulation muscles [111, 96, 97].

\subsection{Introduction}

\subsubsection{Literature review}

Arms with multiarticulation dramatically increase their ability to modulate the end effector stiffness, and multiarticulation is, in fact, necessary to achieve specific joint coupling terms in the desired stiffness [112]. Moreover, a human arm regulates its end-point stiffness by co-contracting uni- and biarticulation muscles to achieve stability under external perturbations $[113,114,115]$. This results in a change of shape and orientation of the work space stiffness ellipsoid that was defined. Here, a clear role of cross-joint couplings was found in shaping this ellipse, and contributing to stability. In [116], the effect of biarticular muscles on the end effector stiffness is investigated, and it was shown that biarticular muscles can stabilize a motion more efficiently and change the direction of a stiffness ellipse. In [117], a new mechanism using a biarticular coupling to extend stiffness ranges is proposed. The influence of biarticulation on the size, shape and orientation of the stiffness ellipse in a two degrees of freedom arm is investigated and it was found that biarticulation indeed extends the possible range, i.e., biarticulation is an additional degree of freedom to shape and orient the work space stiffness ellipse.

There is also an increasing research interest in the stiffness control of compliant manipulators due to the paradigm shift from rigid, fast, and high bandwidth position controlled manipulators, to compliant, slower, and lower-bandwidth interaction controlled manipulators. 
In [118], an impedance control scheme was proposed for a robot with constant joint stiffness, and in [119], the achievable Cartesian stiffness with respect to the number of degrees of freedom is investigated for a manipulator with uncoupled joint compliance. A Frobenius norm was used and minimized to quantify and control the stiffness approximation error in work space for diagonal joint stiffness matrices. The redundant manipulator's null space motion was used to further approximate the desired stiffness. Furthermore, in [120], a control was proposed to achieve a reachable desired work space stiffness ellipse at the end effector, by calculating the necessary actuator setpoints. In [121], a human's end-point stiffness is being replicated in a robotic arm. The common-mode and configuration-dependent stiffness are proposed. The common-mode stiffness is inspired by human muscle control studies that show little dependence of co-contraction on stiffness ellipsoid orientation, while the configurationdependent stiffness changes the orientation of the ellipsoid by changing the posture of the system. A control law is proposed to control the end effector position and orientation while allowing the joint configuration variables to move in the null space to adjust the posture to regulate the stiffness ellipsoid. In line with this, an assessment of the Cartesian stiffness control performance in rigid torque controlled arms is performed in [122]. It was concluded that not in every arm configuration, every stiffness is reachable due to joint torque limitations. A stiffness feasibility region was proposed, which is a result of the arm configuration and joint torque limits due to actuator saturation. The range of the stiffness feasibility region can be extended by using the arm's configuration.

\subsubsection{Contribution}

The contributions of this chapter are mainly twofold. At first, the stiffness modeling and analysis of compliant manipulators modeled using graphs is proposed. This is done by proposing a systematic way of obtaining the actuation Jacobian through fundamental loop matrices on graphs representing manipulators, which is used to map stiffness and compliance between actuation and joint spaces. Although graphs have been proposed in earlier work to model manipulators [82], the structured method obtained here results in a clear mapping how the individual actuator stiffnesses influence each joint stiffness, and is, to the best of the authors' knowledge, not present in the literature. This approach is valid for any actuator connection topology with arbitrary complexity. Furthermore, because the Jacobian maps cannot be inverted in general, and the pseudo-inverse minimizes a norm that is physically not meaningful unless suitable norms are defined on the corresponding vector spaces [123, 124], the stiffness in joint space is mapped as a compliance to the compliance in work space. This is a natural map without (pseudo-)inverting the manipulator's Jacobian. This procedure is experimentally validated on a two degrees of freedom compliant planar manipulator.

The second contribution of this chapter is a stiffness control to achieve a desired work space stiffness at the end effector with actuation stiffnesses that are configured according to a certain topology. In particular, the control of compliant actuators to achieve a desired work space stiffness of the end effector, by optimizing the actuators' topology and their stiffnesses, is treated. A geometry based metric is proposed to quantitatively compare stiffnesses in joint space. Minimizing this metric results in an optimal actuator topology with optimal stiffnesses to achieve or approximate the desired end effector stiffness. This optimization can be interpreted as design guidelines for the design of compliant manipulators. A simulation with a biarticulated two degrees of freedom compliant planar manipulator shows the performance of the optimization with this proposed metric. 


\subsubsection{Stiffness considerations and assumptions}

One may define a stiffness matrix $K$, e.g., created by multiple variable stiffness actuators, to be the Hessian of the potential energy function $V(r)$ with respect to configuration variable $r$ :

$$
K:=\frac{\partial^{2} V(r)}{\partial r^{2}}
$$

When considering a linear stiffness in a region that is small enough, the potential energy function $V(r)$ is quadratic. A generic form can then be given by:

$$
V(r)=\frac{1}{2}\left(r-r_{0}\right)^{\mathrm{T}} K(r)\left(r-r_{0}\right)
$$

where $r_{0}$ is the equilibrium configuration. The Hessian of this potential energy function can be calculated:

$$
\begin{aligned}
\frac{\partial^{2} V(r)}{\partial r^{2}}=K(r) & + \\
& \frac{\partial K(r)}{\partial r}\left(r-r_{0}\right)+\left(r-r_{0}\right) \frac{\partial K(r)}{\partial r}+ \\
& \underbrace{\frac{1}{2}\left(r-r_{0}\right) \frac{\partial^{2} K(r)}{\partial r^{2}}\left(r-r_{0}\right)}_{=0 \text { at equilibrium, where } r-r_{0}} .
\end{aligned}
$$

It can be seen that only in equilibrium, i.e., where $r-r_{0}=0$, is the Hessian of the potential energy function the stiffness tensor [108]. Also, a mechanism can be in equilibrium, while the individual elastic elements are not. When not in equilibrium, so in the presence of preloading, there are additional terms such that the stiffness does not transform tensorially and can become asymmetric $[125,126]$. In the remainder of this chapter, stiffness is modeled as a quasi-static effect at equilibrium, without preloading. This means that stiffnesses are assumed to be symmetric.

Stiffness can be considered as the map from generic displacement change $\delta r$ to force change $\delta F$, i.e.:

$$
K: \delta r \mapsto \delta F .
$$

This $\delta r$ may be represented by an $\dot{r}$ when a time scaling is applied. In this work it is considered that $\delta r$ is an infinitesimal displacement velocity $\dot{r}$ applied for 1 second.

\subsubsection{Outline}

The remainder of the chapter is structured as follows. Section 8.2 presents the stiffness analysis of compliant manipulators modeled on graphs, by introducing an approach to obtain the actuation Jacobian, and by using intrinsic stiffness and compliance mappings. The design, and control of a desired work space stiffness by using a stiffness approximation metric is presented in Section 8.3. The results are discussed in Section 8.4, the chapter concludes with Section 8.5.

\subsection{Mapping Actuation Space Stiffness to Work Space}

To analyze the stiffness of a generic manipulator, one may consider various manifolds, and their connection, representing the state of the manipulator. Figure 8.2 shows a commutative diagram of the various mappings in between actuation, configuration, and work spaces 


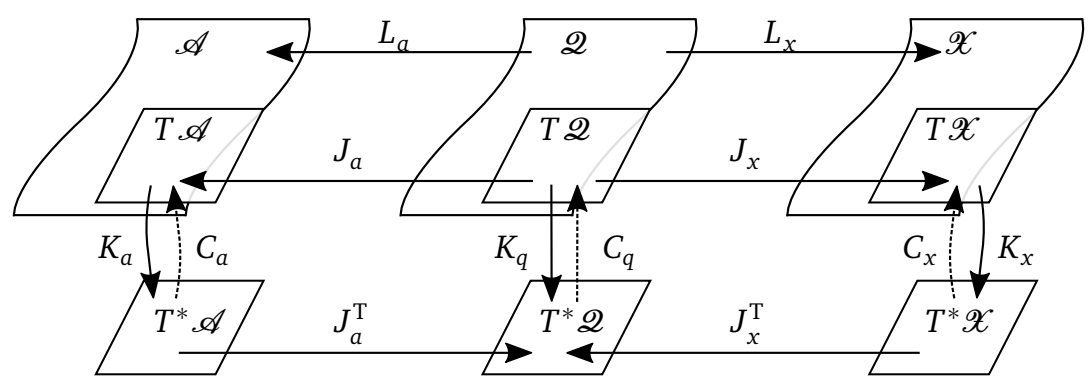

Figure 8.2: Commutative diagram that shows the various stiffness mappings between configuration manifolds $(\mathscr{A}, \mathscr{Q}$, and $\mathscr{X})$, velocity tangent spaces $(T \mathscr{A}, T \mathscr{Q}$, and $T \mathscr{X})$, and the dual force cotangent spaces $\left(T^{*} \mathscr{A}, T^{*} \mathscr{Q}\right.$, and $\left.T^{*} \mathscr{X}\right)$.

and manifolds of a generic actuated mechanism, e.g., a manipulator. The configuration of a rigid body system is often identified by assigning a minimum set of independent generalized configuration coordinates $q_{i} \in \mathscr{Q}$ on manifold $\mathscr{Q}$ to the joints of the system, and using a forward kinematics map $L_{x}$ to calculate the work space configuration of the body or frame under consideration (often the end effector) on work space configuration manifold $\mathscr{X}$. When the configuration of all bodies (and masses, e.g., rotors) is known, the actuation map $L_{a}$ to actuator configuration manifold $\mathscr{A}$ can be defined. Here, actuator configuration manifold may also indicate the configuration manifold of passive elements connected to the joints, e.g., springs and dampers. Velocities are elements of the tangent spaces at a specific element on the manifolds. The configuration velocity $\dot{q} \in \mathscr{T} \mathscr{Q}$ can be mapped to the work space velocity $\dot{x} \in \mathscr{T} \mathscr{X}$ using the manipulator's Jacobian $J_{x}$. Actuators are connected to the joints in a certain way, and their velocity as a function of the configuration velocity $\dot{q}$ is described by the actuation Jacobian $J_{a}$. In a uniarticulation system, where all joints are actuated by one dedicated actuator, the actuation Jacobian $J_{a}$ is diagonal.

In general, the inverse of the Jacobians is used to be able to map a stiffness in joint space $K_{q}$ to a stiffness in work space $K_{x}[113,114,117,120,121]$, by:

$$
K_{x}=J_{x}^{-\mathrm{T}} K_{q} J_{x}^{-1}
$$

This works in case of a non-redundant manipulator that is not in a singular configuration. In that case, the manipulator's Jacobian is square and has full rank, and is, therefore, invertible, which is indeed explicitly assumed in [117]. For general manipulators, the Jacobian is not necessarily square, so inversion is not possible. A pseudo-inversion is possible, but the physical validity depends on a proper metric.

From the commutative diagram in Figure 8.2, it becomes clear that there are four intrinsic mappings, without needing Jacobian inversion, namely the actuation $(a)$ and work space $(x)$ stiffnesses expressed in joint space $(q), K_{a}^{q}$ and $K_{x}^{q}$, respectively, and the joint space compliance expressed in actuation and work space, $C_{q}^{a}$ and $C_{q}^{x}$, respectively. The stiffness mappings are given by:

$$
\begin{aligned}
& K_{q} \rightarrow K_{a}^{q}:=J_{a}^{\mathrm{T}} K_{a} J_{a} \\
& K_{q} \rightarrow K_{x}^{q}:=J_{x}^{\mathrm{T}} K_{x} J_{x},
\end{aligned}
$$

while the compliance mappings are given by

$$
\begin{aligned}
& C_{a} \rightarrow C_{q}^{a}:=J_{a} C_{q} J_{a}^{\mathrm{T}} \\
& C_{x} \rightarrow C_{q}^{x}:=J_{x} C_{q} J_{x}^{\mathrm{T}},
\end{aligned}
$$



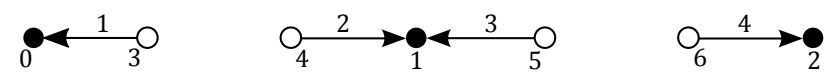

Figure 8.3: A two degrees of freedom manipulator modeled as a graph [91]. The closed vertices represent the bodies, while the open vertices represent the connection points of a kinematic pair to which element like springs and actuators can be connected.

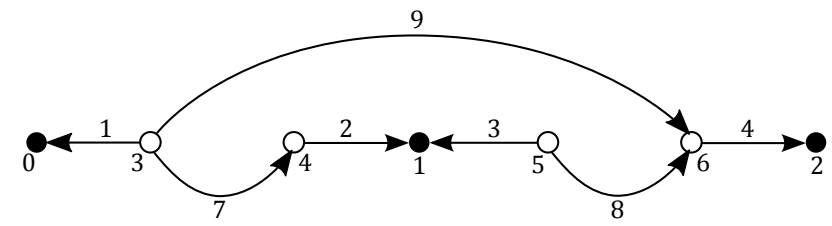

Figure 8.4: A two degrees of freedom manipulator with connected passive springs.

where $K_{i}^{j}$ and $C_{i}^{j}$ are the stiffness and compliance, respectively, of space $i$ expressed in space $j$, and where $C_{i}^{j}=\left(K_{i}^{j}\right)^{-1}$, provided this inversion is possible.

In the remainder of this chapter, stiffnesses in work space are represented with respect to a canonical Cartesian reference frame.

\subsubsection{Passive springs}

In previous work, a model for compliant manipulators was developed based on graphs [91], with which this work proceeds. Here, a systematic way of obtaining the actuation Jacobian from graph models is proposed, with which the stiffnesses of connected passive springs are mapped to joint configuration stiffnesses.

\section{From actuation space to joint space}

Consider the graph of Figure 8.3 as an example. This is the graph of a two degrees of freedom $(n=2)$ manipulator. The open vertices $\mathrm{v}_{3}, \mathrm{v}_{4}, \mathrm{v}_{5}$, and $\mathrm{v}_{6}$ represent the first and second joint, respectively, and they are open for interconnection with various edges representing nodic elements, like springs, dampers, and actuators. These joints transform the connected elements to the six-dimensional space of rigid body motion. The solid vertices represent the bodies of the manipulator $\left(\mathrm{v}_{0}, \mathrm{v}_{1}\right.$, and $\left.\mathrm{v}_{2}\right)$. It is assumed that bodies 0 and 2 are the first and last bodies, respectively, of the chain, and, therefore, have only one interconnection vertex instead of the two as present on the inbetween body 1 . These interconnections are kinematic pairs that define the relative six-dimensional motion between two connected bodies. The edges $\mathrm{E}_{1}, \mathrm{E}_{2}, \mathrm{E}_{3}$, and $\mathrm{E}_{4}$ represent generalized six-dimensional velocities and forces, and are the transformed (possibly lower dimensional) joint velocities and forces, as defined by the kinematic pairs.

In Figure 8.4, graph $\mathfrak{G}$ is shown, where passive springs are connected to the joints to obtain two uniarticulation springs (edges $\mathrm{E}_{7}$ and $\mathrm{E}_{8}$, with stiffnesses $k_{1}$ and $k_{2}$, respectively), and one biarticulation spring (edge $\mathrm{E}_{9}$, with stiffness $k_{3}$ ). With this, a passive compliant two degrees of freedom mechanism is obtained.

In order to relate joint space to actuation space, i.e., to obtain the actuation Jacobian, the spanning tree of graph $\mathfrak{G}$ is necessary. The spanning tree is a new, non-unique graph $\mathfrak{G}_{\text {span }}$, shown in Figure 8.5, with the properties that no vertex is disjoint from the rest of the graph (so every vertex is connected to another vertex), and no loops exist in the graph. It is the minimal graph necessary to connect all vertices, i.e., to span the set of vertices of graph $\mathfrak{G}$. To 


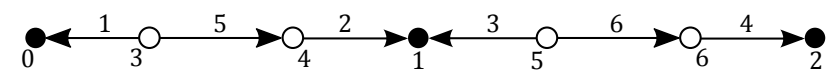

Figure 8.5: The spanning graph of a two degrees of freedom manipulator, with added edges to model the joints.

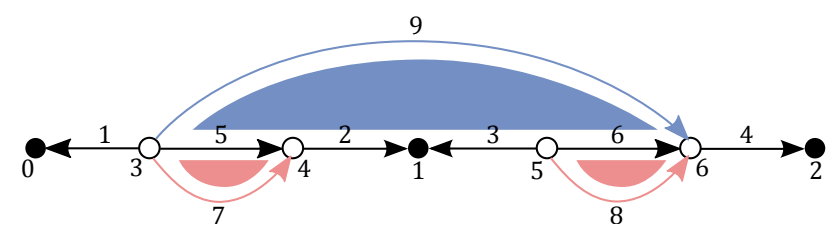

Figure 8.6: The graph of the two degrees of freedom manipulator, with connected springs. The possible loops, defined by the springs, have been drawn in red (uniarticulation) and blue (biarticulation).

relate joint space to actuation space, a convenient choice is to explicitly include the edges that represent joint configurations, and, therefore, the spanning tree was constructed by removing edges $\mathrm{E}_{7}, \mathrm{E}_{8}$, and $\mathrm{E}_{9}$, representing the springs, and introducing edges $\mathrm{E}_{5}$ and $\mathrm{E}_{6}$, representing the joints.

When the passive spring edges are, again, included in the spanning tree graph $\mathfrak{G}_{\text {span }}$, the graph in Figure 8.6 is obtained. Hence, the passive spring edges now create three loops in the graph, defined by a set of edges and shown by shaded areas inside the graph. Each loop consists of only spanning tree graph edges (in black), and one additional edge (the spring edge, in red or blue). Therefore, a loop in this graph can be uniquely identified with a spring edge.

The loops in this graph define a balance law, like the Kirchhoff's Voltage Law in electrical circuits, summing the efforts in a loop to zero:

$$
\begin{aligned}
& l_{1}: e_{\mathrm{E}_{7}}-e_{\mathrm{E}_{5}}=\dot{\theta}_{1}-\dot{q}_{1}=0 \\
& l_{2}: e_{\mathrm{E}_{8}}-e_{\mathrm{E}_{6}}=\dot{\theta}_{2}-\dot{q}_{2}=0 \\
& l_{3}: e_{\mathrm{E}_{9}}-e_{\mathrm{E}_{6}}+e_{\mathrm{E}_{3}}-e_{\mathrm{E}_{2}}-e_{\mathrm{E}_{5}} \\
& =\dot{\theta}_{3}-\dot{q}_{2}+e_{\mathrm{E}_{3}}-e_{\mathrm{E}_{2}}-\dot{q}_{1}=0,
\end{aligned}
$$

where $e_{\mathrm{E}_{i}}$ is the effort of edge $\mathrm{E}_{i}, \theta_{i}$ is the state of the $i^{\text {th }}$ spring in the mechanism, and $q_{i}$ is the $i^{\text {th }}$ joint. In matrix form, this becomes:

$$
\left[\begin{array}{cccc|cc|ccc}
0 & 0 & 0 & 0 & -1 & 0 & 1 & 0 & 0 \\
0 & 0 & 0 & 0 & 0 & -1 & 0 & 1 & 0 \\
0 & -1 & 1 & 0 & -1 & -1 & 0 & 0 & 1
\end{array}\right]\left[\begin{array}{c}
e_{\mathrm{E}_{1}} \\
e_{\mathrm{E}_{2}} \\
e_{\mathrm{E}_{3}} \\
e_{\mathrm{E}_{4}} \\
\dot{q}_{1} \\
\dot{q}_{2} \\
\hline \dot{\theta}_{1} \\
\dot{\theta}_{2} \\
\dot{\theta}_{3}
\end{array}\right]=\left[\begin{array}{l}
0 \\
0 \\
0
\end{array}\right],
$$

where the first matrix is defined as the fundamental loop matrix $Q_{L}$, of which its middle sub-matrix is denoted with $\bar{Q}_{L}$.

The joint velocity between two bodies $\dot{q}$ is a velocity in joint space, while the edges $\mathrm{E}_{1}$, $\mathrm{E}_{2}, \mathrm{E}_{3}$, and $\mathrm{E}_{4}$ define a velocity in work space. Hence, the relation between the joint coordinates and the spring coordinates, i.e., the mapping $J_{a}$ from joint space to actuator space, can 
be obtained from Equation (8.3) by removing the contribution of those edges, leaving only equations as a function of $\dot{q}_{i}$ and $\dot{\theta}_{i}$. Consequently, the mapping $J_{a}$ can be given by:

$$
\begin{gathered}
\dot{\theta}=-\bar{Q}_{L} \dot{q}=J_{a} \dot{q} \\
{\left[\begin{array}{c}
\dot{\theta}_{1} \\
\dot{\theta}_{2} \\
\dot{\theta}_{3}
\end{array}\right]=\left[\begin{array}{ll}
1 & 0 \\
0 & 1 \\
1 & 1
\end{array}\right]\left[\begin{array}{l}
\dot{q}_{1} \\
\dot{q}_{2}
\end{array}\right] .}
\end{gathered}
$$

The stiffness in actuation space is a diagonal matrix of the individual stiffnesses that were connected:

$$
K_{a}=\left[\begin{array}{ccc}
k_{1} & 0 & 0 \\
0 & k_{2} & 0 \\
0 & 0 & k_{3}
\end{array}\right]=K_{k} .
$$

The transformation of the actuation space stiffness to the joint space stiffness is given by Equation (8.1), and becomes:

$$
\begin{aligned}
K_{a}^{q} & =J_{a}^{\mathrm{T}} K_{a} J_{a} \\
& =\left[\begin{array}{lll}
1 & 0 & 1 \\
0 & 1 & 1
\end{array}\right]\left[\begin{array}{ccc}
k_{1} & 0 & 0 \\
0 & k_{2} & 0 \\
0 & 0 & k 3
\end{array}\right]\left[\begin{array}{ll}
1 & 0 \\
0 & 1 \\
1 & 1
\end{array}\right] \\
& =\left[\begin{array}{cc}
k_{1}+k_{3} & k_{3} \\
k_{3} & k_{2}+k_{3}
\end{array}\right],
\end{aligned}
$$

where $K_{a}^{q}$ is the actuation space stiffness expressed in joint space, which is indeed the stiffness matrix of the familiar two degrees of freedom compliant mechanism with biarticulation.

\section{From joint space to work space}

Mapping a stiffness in joint space to work space is not possible without using the inverse of, in general non-invertible, manipulator Jacobian matrices. The intrinsic way of such a mapping is to map the compliance in joint space to a compliance in work space, given by $C_{q}^{x}$ in Equation (8.2). The compliance in joint space $\left(C_{q}\right.$ in Figure 8.2), which is actually created by the connection of the springs in actuation space, can be obtained by inverting the joint stiffness ( $K_{q}$ in Figure 8.2), in particular the one of Equation (8.4). This inversion is valid since it is a square matrix, and of full rank due to the number of actuators being equal to or larger than the number of joints, i.e., the joints are not underactuated. If this condition is not satisfied, e.g., only one biarticulation is implemented on the two joints, the resulting inverse is not well-defined, i.e., it will be infinite (a zero stiffness is an infinite compliance).

When the manipulator Jacobian $J_{x}$ is obtained, mapping joint to end effector velocities, i.e., $\dot{x}=J_{x} \dot{q}$, the work space compliance $C_{q}^{x}$, i.e., the joint compliance $q$ expressed in work space $x$, can be defined by the mapping given in Equation (8.2). If it is of full rank, the inverse may be taken to obtain the work space stiffness $K_{q}^{x}$.

\subsubsection{Variable stiffness actuators}

\section{From actuation space to joint space}

The use of compliant actuators in manipulators raises the issue of backdrivability. A variable series elastic actuator like the ideal model shown in Figure 8.1 is backdrivable because there 


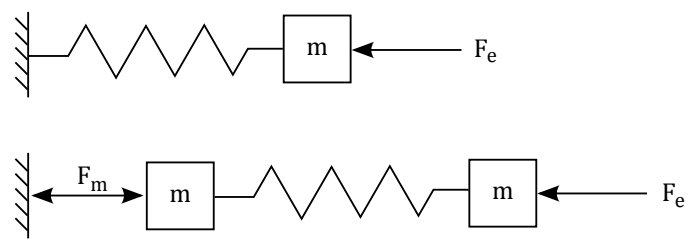

Figure 8.7: Schematic views of a passive compliant joint (top), and an actuated series compliant joint (bottom).

is no mechanical linkage between the stator and rotor that directly transfers forces from one to the other in the direction of the relative motion. The only force in between the rotor and stator is the motor force. Therefore, any external force on the output along the direction of actuation is transferred to the rotor, and will not be transmitted to the stator, due to the absence of this linkage. This is schematically depicted in Figure 8.7. In the top situation, the external force $F_{e}$ will be in equilibrium with the force in the spring, in a stationary situation, and that force acts on the fixed base. In the bottom situation, the force $F_{e}$ is not transferred beyond the left mass, so the fixed base does not experience this force, and, therefore, the stiffness perceived when applying force $F_{e}$ will be zero in a backdrivable situation. In case the actuator is non-backdrivable, e.g., by a mechanical non-backdrivable coupling between the rotor and stator, by a control on $F_{m}$ to provide the same force as generated by the elastic element on the rotor, or by a proper rotor position control, the rotor becomes transparent (and basically fixed to the stator). Then, the force $F_{e}$ also acts on the stator and fixed base. In that case, the bottom situation becomes identical to the top situation. In the remainder, it is assumed that a non-backdrivable situation is obtained.

If variable stiffness actuators are used to actuate the same example mechanism of Figure 8.3, and the same considerations as before regarding the spanning tree hold, the fundamental loop graph of the mechanism actuated with two uniarticulation actuators and one biarticulation actuator can be obtained, as shown in Figure 8.8. Again the black edges form the spanning tree, while the red (uniarticulation) and blue (biarticulation) edges identify fundamental loops in the graph.

It can be seen that, because of the inclusion of $m$ VSAs, additional vertices appear in the graph (vertices $\mathrm{v}_{7}, \mathrm{v}_{8}$, and $\mathrm{v}_{9}$ ), that represent the VSA rotors. Because joint coordinates are defined as the minimum set of independent coordinates necessary to completely represent the state of a mechanism, these rotor coordinates should also be included in joint space. Their configuration in joint space relative to its stator is identified with $q_{u_{i}}$. In actuation space the rotor's configuration relative to the stator is $\theta_{u_{i}}$. So, if the same units and norm on the spaces are chosen, it holds that:

$$
\theta_{u_{i}}=q_{u_{i}}
$$

These rotor vertices are spanned in the spanning tree by edges $\mathrm{E}_{7}, \mathrm{E}_{8}$, and $\mathrm{E}_{9}$, representing the coupling between the VSA's stator and rotor. Edges $\mathrm{E}_{10}, \mathrm{E}_{11}$, and $\mathrm{E}_{12}$ are the elastic elements within the VSAs ${ }^{1}$.

\footnotetext{
${ }^{1}$ It is noted that the direction of these edges has reversed with respect to previous work [91]. This is because a different convention for the constitutive equation of the elastic element was used, $\delta F=-k \delta r$ instead of $\delta F=k \delta r$, leading to a more intuitive joint space to actuator space relation.
} 


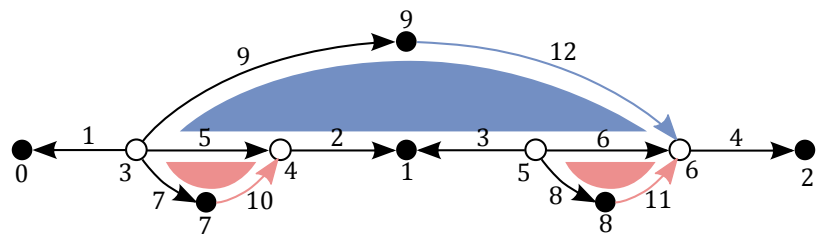

Figure 8.8: The graph of the two degrees of freedom manipulator, with connected actuators. The possible loops, defined by the actuators, have been drawn in red (uniarticulation actuators) and blue (biarticulation actuator).

The loop equations can be found again:

$$
\begin{aligned}
& l_{1}: e_{\mathrm{E}_{10}}-e_{\mathrm{E}_{5}}+e_{\mathrm{E}_{7}}=\dot{\theta}_{1}-\dot{q}_{1}+\dot{q}_{u_{1}}=0 \\
& l_{2}: e_{\mathrm{E}_{11}}-e_{\mathrm{E}_{6}}+e_{\mathrm{E}_{8}}=\dot{\theta}_{2}-\dot{q}_{2}+\dot{q}_{u_{2}}=0 \\
& l_{3}: e_{\mathrm{E}_{12}}-e_{\mathrm{E}_{6}}+e_{\mathrm{E}_{3}}-e_{\mathrm{E}_{2}}-e_{\mathrm{E}_{5}}+e_{\mathrm{E}_{9}} \\
& \quad=\dot{\theta}_{3}-\dot{q}_{2}+e_{\mathrm{E}_{3}}-e_{\mathrm{E}_{2}}-\dot{q}_{1}+\dot{q}_{u_{3}}=0 .
\end{aligned}
$$

In matrix form, this becomes:

$$
\begin{aligned}
& {\left[\begin{array}{cccc|ccccc|ccc}
0 & 0 & 0 & 0 & -1 & 0 & 1 & 0 & 0 & 1 & 0 & 0 \\
0 & 0 & 0 & 0 & 0 & -1 & 0 & 1 & 0 & 0 & 1 & 0 \\
0 & -1 & 1 & 0 & -1 & -1 & 0 & 0 & 1 & 0 & 0 & 1
\end{array}\right]\left[\begin{array}{c}
e_{\mathrm{E}_{1}} \\
e_{\mathrm{E}_{2}} \\
e_{\mathrm{E}_{3}} \\
e_{\mathrm{E}_{4}} \\
\hline \dot{q}_{1} \\
\dot{q}_{2} \\
\dot{q}_{u_{1}} \\
\dot{q}_{u_{2}} \\
\dot{q}_{u_{3}} \\
\hline \dot{\theta}_{1} \\
\dot{\theta}_{2} \\
\dot{\theta}_{3}
\end{array}\right] } \\
&=\left[\begin{array}{c}
0 \\
0 \\
0
\end{array}\right],
\end{aligned}
$$

where columns 5 and 6 are denoted as sub-matrix $\bar{Q}_{L}$.

With this, and using Equation (8.5), the actuation Jacobian can be obtained:

$$
\begin{aligned}
\dot{\theta}^{\prime} & =J_{a} \dot{q}^{\prime} \\
{\left[\begin{array}{c}
\dot{\theta}_{u_{1}} \\
\dot{\theta}_{u_{2}} \\
\dot{\theta}_{u_{3}} \\
\dot{\theta}_{1} \\
\dot{\theta}_{2} \\
\dot{\theta}_{3}
\end{array}\right] } & =\left[\begin{array}{ccccc}
0 & 0 & 1 & 0 & 0 \\
0 & 0 & 0 & 1 & 0 \\
0 & 0 & 0 & 0 & 1 \\
1 & 0 & -1 & 0 & 0 \\
0 & 1 & 0 & -1 & 0 \\
1 & 1 & 0 & 0 & -1
\end{array}\right]\left[\begin{array}{c}
\dot{q}_{1} \\
\dot{q}_{2} \\
\dot{q}_{u_{1}} \\
\dot{q}_{u_{2}} \\
\dot{q}_{u_{3}}
\end{array}\right] \\
& =\left[\begin{array}{ccc}
0_{m \times n} & I_{m \times m} \\
-\bar{Q}_{L} & -I_{m \times m}
\end{array}\right]\left[\begin{array}{c}
\dot{q}_{1} \\
\dot{q}_{2} \\
\dot{q}_{u_{1}} \\
\dot{q}_{u_{2}} \\
\dot{q}_{u_{3}}
\end{array}\right],
\end{aligned}
$$

where $0_{m \times n}$ is a zeros matrix of size $(m \times n)$, and $I_{m \times m}$ is the identity matrix of size $(m \times m)$, with $m$ the number of compliant actuators, and $n$ the number of manipulator degrees of freedom. 
The stiffness in actuation space is again a diagonal matrix of the passive stiffnesses of the elastic elements $k_{i}$, but now also of the actively generated stiffnesses $\kappa_{i}$, controlled by the force input between the VSA's stator and rotor. It is given by:

$$
\begin{aligned}
K_{a} & =\left[\begin{array}{cccccc}
\kappa_{1} & 0 & 0 & 0 & 0 & 0 \\
0 & \kappa_{2} & 0 & 0 & 0 & 0 \\
0 & 0 & \kappa_{3} & 0 & 0 & 0 \\
0 & 0 & 0 & k_{1} & 0 & 0 \\
0 & 0 & 0 & 0 & k_{2} & 0 \\
0 & 0 & 0 & 0 & 0 & k_{3}
\end{array}\right] \\
& =\left[\begin{array}{cc}
K_{\kappa} & 0_{m \times m} \\
0_{m \times m} & K_{k}
\end{array}\right] .
\end{aligned}
$$

Again, the actuation space stiffness transformed to and expressed in joint space $K_{a}^{q}$, is then given by:

$$
\begin{aligned}
K_{a}^{q} & =J_{a}^{\mathrm{T}} K_{a} J_{a} \\
& =\left[\begin{array}{ccccc}
k_{1}+k_{3} & k_{3} & -k_{1} & 0 & -k_{3} \\
k_{3} & k_{2}+k_{3} & 0 & -k_{2} & -k_{3} \\
-k_{1} & 0 & \kappa_{1}+k_{1} & 0 & 0 \\
0 & -k_{2} & 0 & \kappa_{2}+k_{2} & 0 \\
-k_{3} & -k_{3} & 0 & 0 & \kappa_{3}+k_{3}
\end{array}\right],
\end{aligned}
$$

which results in a stiffness in block-matrix form, using (8.6) and (8.7), for generic actuator topologies:

$$
K_{a}^{q}=\left[\begin{array}{cc}
\bar{Q}_{L}^{\mathrm{T}} K_{k} \bar{Q}_{L} & \bar{Q}_{L}^{\mathrm{T}} K_{k} \\
K_{k} \bar{Q}_{L} & K_{\kappa}+K_{k}
\end{array}\right] .
$$

\section{From joint space to work space}

Similar to the situation with only passive springs, this stiffness matrix is square and has full rank, because of the additional active stiffnesses $\kappa_{i}$. Therefore, the inverse can be taken to find the compliance in joint space. Then, by using the manipulator Jacobian, the compliance in joint space can be mapped to the compliance in work space $\left(C_{q}^{x}\right)$, according to Equation (8.2), and if it is of full rank, the inverse may be taken to obtain the work space stiffness $K_{q}^{x}$. Because of the presence of additional configuration variables $q_{u_{i}}$, the Jacobian map as discussed in Section 8.2.1 changes according to:

$$
\begin{aligned}
\dot{x} & =J_{x}^{\prime} \dot{q}^{\prime} \\
\dot{x} & =J_{x}^{\prime}\left[\begin{array}{llllll}
\dot{q}_{1} & \ldots & \dot{q}_{n} & \dot{q}_{u_{1}} & \ldots & \dot{q}_{u_{m}}
\end{array}\right]^{\mathrm{T}} \\
J_{x}^{\prime} & =\left[\begin{array}{lllll}
J_{x} & 0_{1} & \ldots & 0_{m}
\end{array}\right],
\end{aligned}
$$

where $0_{1} \ldots 0_{m}$ are $m$ columns with elements that are zero.

\subsubsection{Experimental validation: biarticulated planar mechanism}

To validate the stiffness modeling as proposed in the previous sections, an experiment was done with a two degrees of freedom planar manipulator, shown in Figure 8.9a. It consists of 


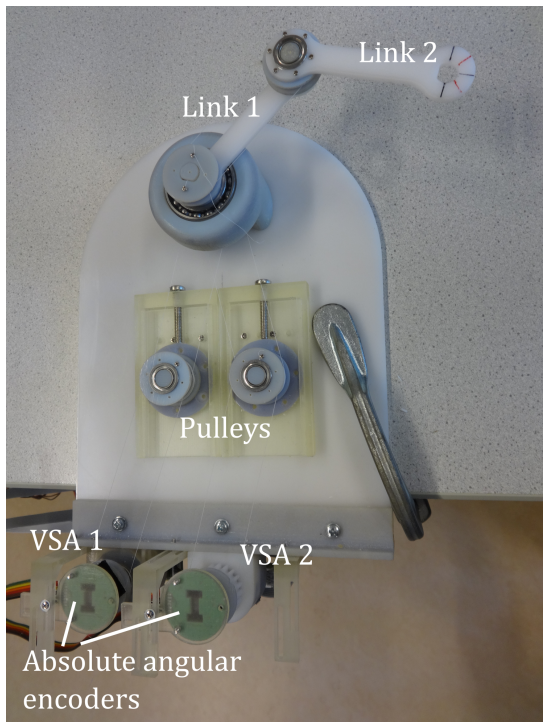

(a) Photo of the measurement setup.

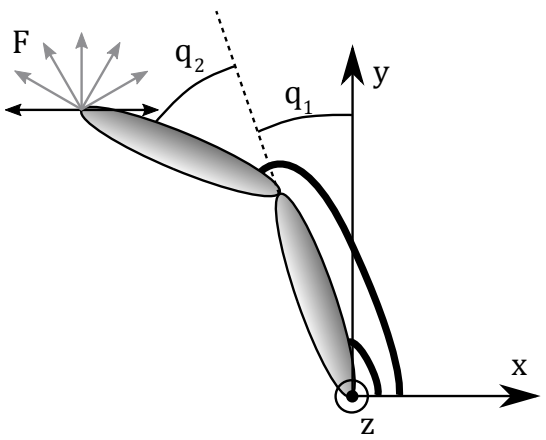

(b) Schematic view of the measurement setup with one uniarticulation and one biarticulation VSA connected.

Figure 8.9: Measurement setup of a two degree of freedom planar manipulator.

two links of $6 \mathrm{~cm}$ length that are driven by variable stiffness actuators (VSA 1 and VSA 2, as presented in [51]). These actuators are connected to the links by tendons and pulleys, which allows a VSA to be coupled in a biarticulation way (spanning two joints).

For this experiment, one VSA was coupled to the first joint (uniarticulation), while another VSA was connected to both joints (biarticulation), as schematically shown in Figure 8.9b. This results in a graph that is identical to the one shown in Figure 8.8, apart from vertex $\mathrm{v}_{8}$, and edges $E_{8}$ and $E_{11}$ being removed. This results in an actuation Jacobian given by:

$$
J_{a}=\left[\begin{array}{cccc}
0 & 0 & 1 & 0 \\
0 & 0 & 0 & 1 \\
1 & 0 & -1 & 0 \\
1 & 1 & 0 & -1
\end{array}\right]
$$

which is obtained by using the procedure as described previously. It can be seen that this actuation Jacobian is basically the one in Equation (8.6), where the second and fourth row have been removed, as well as the fourth column, removing the presence of the uniarticulation actuator. The manipulator was put in a configuration, and the stiffnesses of the VSAs were held constant at a specific value:

$$
K_{a}=\left[\begin{array}{cccc}
\kappa_{1} & 0 & 0 & 0 \\
0 & \kappa_{2} & 0 & 0 \\
0 & 0 & k_{1} & 0 \\
0 & 0 & 0 & k_{2}
\end{array}\right]
$$

A spring scale was used to apply a small force of $F=0.3 \mathrm{~N}$ to the end effector in directions ranging from $0^{\circ}$ to $180^{\circ}$ with respect to the positive x-axis as shown in Figure 8.9b, in steps of $10^{\circ}$. The deflection of the manipulator was measured at the actuators by absolute magnetic 


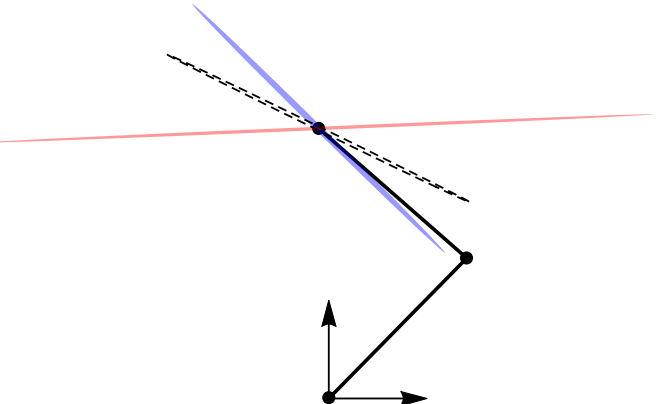

(a) $k_{1}=0.02 \mathrm{Nm} / \mathrm{rad}$ and $k_{2}=1.1 \mathrm{Nm} / \mathrm{rad}$.

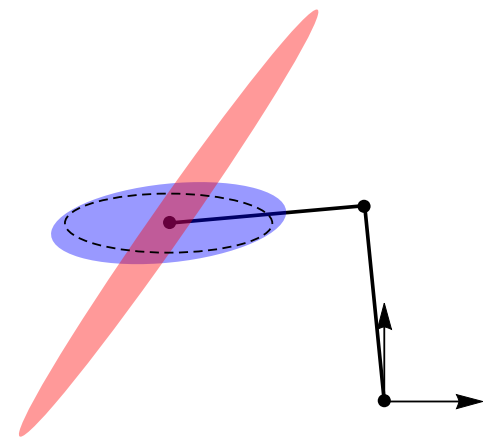

(b) $k_{1}=0.02 \mathrm{Nm} / \mathrm{rad}$ and $k_{2}=0.06 \mathrm{Nm} / \mathrm{rad}$.

Figure 8.10: Two different manipulator configurations with theoretical end effector compliance (blue shaded ellipse) and measured compliance (black dashed ellipse). The specific VSA stiffness settings are given in the corresponding captions. In (a) a high stiffness ratio was chosen to make the directional property more explicit. The red ellipse shows the compliance in case the VSAs were connected in a uniarticulation way.

encoders. This has the advantage of bypassing the relatively compliant tendons that may influence the measurement. The end effector deflection is then estimated, which is possible in this specific case because the actuation Jacobian $J_{a}$ in Equation (8.11) is invertible such that the mapping $J_{x} J_{a}^{-1}: \delta \theta \mapsto \delta x$, to map actuation space displacements $\delta \theta$ to work space displacements $\delta x$, is valid. By straightforward geometry, the forward kinematics map can be obtained, which is differentiated to yield the manipulator Jacobian:

$$
\begin{aligned}
& J_{x}= \\
& {\left[\begin{array}{cccc}
0 & 0 & 0 & 0 \\
0 & 0 & 0 & 0 \\
1 & 1 & 0 & 0 \\
-l_{1} \cos \left(q_{1}\right)-l_{2} \cos \left(q_{1}+q_{2}\right) & -l_{2} \cos \left(q_{1}+q_{2}\right) & 0 & 0 \\
-l_{1} \sin \left(q_{1}\right)-l_{2} \sin \left(q_{1}+q_{2}\right) & -l_{2} \sin \left(q_{1}+q_{2}\right) & 0 & 0 \\
0 & 0 & 0 & 0
\end{array}\right] .}
\end{aligned}
$$

A compliance ellipse was estimated using a least-squares fit to the force-deflection points. Modeling the end effector compliance is done according to Equations (8.1) and (8.2), which is compared to the measurement and the compliance estimate. This is shown in Figure 8.10 for two different configurations.

The theoretical compliance at the end effector is indicated with a blue shaded ellipse, while the measurements are indicated with a black dashed ellipse. It can be seen that the measured compliance matches the size of the theoretical compliance. The orientation of the ellipses in Figure $8.10 \mathrm{~b}$ is also equal, while the orientation in Figure 8.10a is slightly deviated. This can be due to a slight deviation of the tested configuration or the actuator stiffnesses that are set. However, the method to obtain the actuation Jacobian can be considered valid. Moreover, a red ellipse can be seen, indicating the compliance when the two VSAs would be connected in a uniarticulation way. The biarticulation property in the setup is, therefore, also explicitly shown. 


\subsection{Controlling the Desired Work Space Stiffness}

The previous section considers a predefined actuation topology on a compliant manipulator. However, the topology is a degree of freedom in the design space of a manipulator. Very often uniarticulation actuators are chosen ad hoc, since this is easily mechanically realizable. The control is then relatively straightforward since there is no cross-joint coupling. However, when the objective is to approximate a desired work space stiffness with a work space stiffness that is obtained from the connected actuators, an ad hoc choice for uniarticulation might not be the best option, and various actuation topologies and varying actuator stiffnesses should be examined. A different actuation topology creates a different stiffness that may be perceived, and it may, or may not, yield a better approximation to the desired work space stiffness. Graphs are useful to analyze these differences, since a manipulator's actuation topology can be easily adapted.

\subsubsection{Approximation method}

To quantify this approximation, one could transform the stiffness in actuation space to the stiffness in work space and relate the two stiffness matrices. However, this mapping cannot be done, in general, without inverting Jacobian mappings that might not be invertible. Therefore, it is proposed here to map both the work space stiffness as well as the actuation space stiffness to stiffnesses in joint space, which can be done without inversion, as shown in Equation (8.1). The two joint stiffness matrices can then be quantitatively compared to assess the approximation in work space.

The Jacobian of a manipulator actuated by VSAs is of the form as given in Equation (8.10), i.e., with as many zero-columns $(m)$ as the number of VSAs. The desired work space stiffness $K_{x \text { des }}$ can be transformed to a desired joint space stiffness $K_{x}^{q}$, using this Jacobian, by:

$$
\begin{aligned}
K_{x}^{q} & =J_{x}^{\prime \mathrm{T}} K_{x \text { des }} J_{x}^{\prime} \\
& =\left[\begin{array}{llll}
J_{x} & 0_{1} & \ldots & 0_{m}
\end{array}\right]^{\mathrm{T}} K_{x \text { des }}\left[\begin{array}{llll}
J_{x} & 0_{1} & \ldots & 0_{m}
\end{array}\right] \\
& =\left[\begin{array}{ll}
K_{x \text { des }}^{\prime} & 0_{n \times m} \\
0_{m \times n} & 0_{m \times m}
\end{array}\right] .
\end{aligned}
$$

The matrix obtained here should be approximated by the matrix $K_{a}^{q}$ in (8.9), by choosing an actuator connection topology, given by $\bar{Q}_{L}$, and setting the right stiffnesses. In case of a backdrivable system, where $\dot{q}_{u_{i}} \neq 0$, this approximation is never exact due to the block matrices $\bar{Q}_{L}^{\mathrm{T}} K_{k}, K_{k} \bar{Q}_{L}$, and $K_{\kappa}+K_{k}$, in Equation (8.9), being non-zero. When a non-backdrivable situation is considered, it holds that $\dot{q}_{u_{i}}=0$, and then the comparison between $K_{x \text { des }}^{\prime}$ and $\bar{Q}_{L}^{\mathrm{T}} K_{k} \bar{Q}_{L}$ in Equation (8.9) is sufficient.

When viewing it from a purely numerical perspective, $K_{x \text { des }}^{\prime}$ is an $(n \times n)$ matrix, and since it is symmetric, it may have $N=\frac{1}{2} n(n+1)$ independent elements (the diagonal plus the upper triangular part). If $\bar{Q}_{L}^{\mathrm{T}} K_{k} \bar{Q}_{L}$ also has $N$ independent elements, due to a fully overactuated system using all uni- and multiarticulation actuator connections that are possible ( $N$ in total), the desired stiffness can be exactly achieved. If it is not desired to have a fully overactuated system, less actuators may be used, which decreases the approximation quality. Therefore, this analysis also aids in designing the actuation topology, because the quantification of the approximation quality may very explicitly favor one topology over the other. 


\subsubsection{Stiffness Approximation Metric}

Quantifying the approximation of the actuator stiffness to the desired work space stiffness is done in joint space, so that no non-intrinsic mappings have to be used. The Stiffness Approximation Metric (SAM) is proposed here, which quantifies how close an approximation is. Various matrix norms are possible [127], such as an induced norm, and an entry-wise norm. An induced norm is a norm that is induced from a norm on the underlying vector space, and, loosely speaking, it measures the lengthening of vector by the linear map. Here, the associated vector spaces are the joint configuration velocities and forces. However, a norm on those spaces is generally not physically consistent, since the joint configuration velocities may be of different nature, e.g., rotation and translation, and unit, e.g., rad/s and m/s, resulting in physically meaningless quantities and units $[123,124]$. An entry-wise norm, like the Frobenius norm, treats a matrix or map as a collection of numbers in the form of a vector. A regular vector norm may then be applied. Because this norm is entry-wise and it does not consider physical units or structure, it looses the connection with physics.

Here a geometry based metric is proposed. Each matrix may be represented by its singular value decomposition, that geometrically is a rotation of a unit sphere, assuming that sphere has been defined, a scaling of its principle axes, and again a rotation, resulting in an ellipsoid, e.g., the ellipsoid of a stiffness map. This ellipsoid is defined by its principal axes, where the semi-axes have length equal to the singular values. Two stiffness maps can be compared by the geometric property of ellipsoid overlap, where this overlap can be expressed as the similarity of singular values. However, eigenvalues are calculated and used to compare similarity between geometric ellipsoids instead of singular values, because a singular value decomposition is in general more expensive to compute [128].

The proposed Stiffness Approximation Metric is defined as:

$$
\mu:=\left[\operatorname{eig}\left(K_{a}^{q-1} K_{x \text { des }}^{q}-I\right)\right]^{\mathrm{T}}\left[\operatorname{eig}\left(K_{a}^{q-1} K_{x \text { des }}^{q}-I\right)\right],
$$

where $I$ is the identity matrix of appropriate dimensions, i.e., $I \in \mathbb{R}^{(n+m) \times(n+m)}$.

The matrix $K_{a}^{q-1} K_{x \text { des }}^{q}$ is a linear map from the joint configuration velocities vector space to itself. Therefore, an element from that space is mapped to another element in that space. If the two stiffness matrices are identical, the map would yield the identity map, and the map applied to an element in the vector space would return the very same element. In that case, $K_{a}^{q-1} K_{x \text { des }}^{q}-I$ will yield the map zero, with eigenvalues being zero. The result of the calculation of this metric for identical stiffness matrices would then be zero, while any deviation of stiffness maps results in a positive metric value. The stiffness matrix $K_{a}^{q}$ is inverted because it can be assumed invertible, as previously discussed regarding (8.8) and (8.9), while that assumption does not hold for $K_{x}^{q}$.

Minimizing the metric of (8.12) with respect to the stiffness matrices, and, therefore, the individual actuator stiffnesses $k_{1}, k_{2}, \ldots, k_{m}$, results in the optimal stiffness approximation.

\subsubsection{Simulations with a two degrees of freedom manipulator}

As a case study to examine the performance of this Stiffness Approximation Metric, simulations with a two degrees of freedom planar manipulator as shown in Figure 5.11 are done.

\section{Method}

The manipulator is required to move its end effector along a predefined trajectory. During this trajectory, a desired end effector stiffness is imposed that should be achieved or approximated 
by the VSAs driving the manipulator. The stiffness in the VSAs is controlled by the Stiffness Approximation Metric.

For these simulations, a sinusoidal end effector trajectory was chosen. The desired end effector work space stiffness along this trajectory $K_{\text {des }}(t)$ was chosen to be isotropic, i.e., an equal stiffness in all directions. The initial and final desired stiffnesses, $K_{\mathrm{des}}\left(t_{0}\right)$ at initial time $t_{0}$ and $K_{\mathrm{des}}\left(t_{f}\right)$ at final time $t_{f}$, respectively, were chosen to be slightly differently oriented ellipses. The generic form of the desired end effector stiffness in work space is given by

$$
K_{\mathrm{des}}(t)=\left[\begin{array}{cccccc}
0 & 0 & 0 & 0 & 0 & 0 \\
0 & 0 & 0 & 0 & 0 & 0 \\
0 & 0 & 0 & 0 & 0 & 0 \\
0 & 0 & 0 & k_{a} & k_{b} & 0 \\
0 & 0 & 0 & k_{c} & k_{d} & 0 \\
0 & 0 & 0 & 0 & 0 & 0
\end{array}\right],
$$

which defines a non-zero stiffness in $x$ and $y$ translational directions. Specifically, the stiffnesses along the trajectory are given by

$$
\begin{gathered}
K_{\mathrm{des}}\left(t_{0}\right): K_{\mathrm{des}}(t) \mid k_{a}=175, k_{b}=75, k_{c}=75, k_{d}=250 ; \\
K_{\mathrm{des}}\left(t_{f}\right): K_{\mathrm{des}}(t) \mid k_{a}=200, k_{b}=150, k_{c}=150, k_{d}=350 ; \\
K_{\mathrm{des}}\left(t^{\prime}\right): K_{\mathrm{des}}(t) \mid k_{a}=150, k_{b}=0, k_{c}=0, k_{d}=150, \\
\text { with units in } \mathrm{N} / \mathrm{m}, \text { for } t_{0}<t^{\prime}<t_{f} .
\end{gathered}
$$

This stiffness was then mapped to joint space using the manipulator's Jacobian as given in Equation (8.10).

The metric was applied to control the VSA stiffnesses at 12 different points on the sinusoidal trajectory, and, therefore, in 12 different manipulator configurations. This was repeated for all possible actuator topologies with at least two actuators, to prevent underactuation, resulting in four different topologies. Upon the minimization of the metric, solution constraints were applied in which stiffnesses were either required to be zero (in case a VSA is removed from the topology), or were required to be in the reachable stiffness range of the VSAs $(0.01 \mathrm{Nm} / \mathrm{rad} \ldots 1.1 \mathrm{Nm} / \mathrm{rad})$.

\section{Results}

The results of these simulations with the four different actuator topologies can be seen in Figure 8.11. A reference frame $(x, y)$ can be seen, which is the base of the manipulator. The sinusoidal trajectory is shown as a solid black curve, which is reached by the end effector of the manipulator. The manipulator itself is shown in black in the initial configuration, as well

Table 8.1: Cummulative cost of the approximation error as calculated by the Stiffness Approximation Metric, calculated as the sum of the costs of each approximation along the complete trajectory. Stiffness $k_{1}$ and $k_{2}$ are uniarticulation stiffnesses on the first and second joint, respectively, while $k_{3}$ is a biarticulation stiffness. Configurations are indicated with letters corresponding to the figures in Figure 8.11.

\begin{tabular}{ccccc}
\hline Configuration & $k_{1}$ & $k_{2}$ & $k_{3}$ & Cummulative cost $\mu$ \\
\hline a & $\checkmark$ & $\checkmark$ & $\checkmark$ & 0.25 \\
b & $\checkmark$ & $\times$ & $\checkmark$ & 3.05 \\
c & $\times$ & $\checkmark$ & $\checkmark$ & 6.81 \\
d & $\checkmark$ & $\checkmark$ & $\times$ & 10.37 \\
\hline
\end{tabular}


as in some subsequent configurations in faded grey. Along the trajectory, 12 locations with two ellipses at each location are shown. The dashed blue ellipses are the desired stiffnesses at the end effector, as defined in (8.13) and (8.14). The shaded blue ellipses are the achieved stiffnesses by the VSAs, where their stiffnesses are controlled by the Stiffness Approximation Metric. Moreover, straight lines can be distinguished in each ellipse. These are the output force directions upon applying an input displacement in either the $x$-direction (pink), or the $y$-direction (brown), which are a property of the realized stiffness. Dashed lines are obtained from the desired stiffness, while solid lines are obtained from the achieved stiffness.

The actuator topology where the achieved stiffnesses show the closest match to the desired stiffnesses along the trajectory is clearly the optimal topology for this trajectory and desired stiffnesses. The cost of using each of these actuator topologies to achieve the desired stiffnesses is shown in Table 8.1. The fully actuated manipulator with twice uniarticulation and biarticulation has the lowest cost. Therefore, to accurately reproduce the desired stiffness, it is recommended to use the fully actuated topology, as shown in Figure 8.11a. If one VSA should be removed from the topology, due to design constraints for example, then removing the uniarticulation VSA on the second joint, obtaining a system like the one shown in Figure $8.9 \mathrm{~b}$, results in the lowest possible cost, with which an acceptable approximation is still achieved, as shown in Figure 8.11b. If either one of the other VSAs would be removed instead, i.e., the VSA on the first joint, or the biarticulation VSA, the approximation of the achieved stiffness to the desired stiffness becomes worse to the point that using these topologies is not feasible. This is shown in Figures 8.11c and 8.11d.

For a predetermined actuation topology, the controller finds the optimal actuator stiffnesses to approximate the desired stiffness. However, if the actuation topology is still a design parameter, one can interpret these results as design guidelines to aid in the optimal design of a compliant manipulator, by choosing the topology that yields an acceptable approximation.

\subsection{Discussion}

This chapter has treated the description of stiffness and compliance of robotic manipulators modeled using graphs. A structured way of obtaining the actuation Jacobian was proposed by using fundamental loop equations. Using this Jacobian, the stiffness in actuation space, created by the VSAs, was mapped to a stiffness in joint space. The actively controlled stiffnesses $\kappa_{i}$, as controlled by the input force between stator and rotor, were considered to be infinitely stiff to assume a non-backdrivable situation. However, this active compliance may be used to overcome physical actuator limitations like a limited control bandwidth resulting in non-zero stiffness variation time and stiffness range limitations, so that it works in conjunction with the physical stiffness [129].

In Section 8.3 a metric was proposed that can optimize actuator stiffnesses given a certain trajectory. This can be interpreted as design guidelines for a compliant manipulator. These guidelines propose which actuator topology may be used, and what the consequence is in terms of stiffness approximation deterioration when deviating from the optimal topology. An aspect that was not considered in the optimization was the design complexity. Although the optimization might recommend multiarticulation, one may choose not to do so because that may simplify the mechanical design considerably. It has the consequence that the approximation to the desired stiffness is worse and may even be unacceptable. Quantifying this is hardly possible, since it depends on the specific realizations of the various articulation types.

Another important aspect that was left out is the fact that the end effector work space stiffness is configuration dependent. In case a manipulator is kinematically redundant, the redundant degrees of freedom can be used for other purposes, e.g., for achieving a certain 
end effector stiffness, like done in [121]. The system that is considered here is not kinematically redundant, which means that the configuration of the manipulator cannot be used to achieve a desired stiffness while tracking an end effector trajectory. Moreover, in this work, achieving the end effector trajectory takes precedence over achieving a desired stiffness, i.e., the manipulator configuration is a constraint in the behavior and optimization of the system. Future work may focus on a kinematically redundant manipulator, where the optimization might not be only for the stiffness and its topology, but also for the configuration.

The metric used here is based on geometric considerations and the results when it is used in the optimization are plausible. However, and obviously, a different metric will give a different result. Future investigations may consider different metrics and the comparison with the one proposed here. Moreover, the metric should be minimized globally and not locally. The numeric solver should ensure that a global minimum is found, for instance by appropriately randomizing initial conditions to have a large search space. Ensuring this is beyond the scope of this work.

The setup used in Section 8.2 was not accurate and robust enough to yield acceptable stiffness control measurements. Therefore, future work should most of all focus on the preparation of a robust experimental setup to properly validate the stiffness control as proposed here.

\subsection{Conclusion}

In this chapter, the stiffness of compliant manipulators that are modeled using graphs was described. The structured approach for obtaining the actuation Jacobian by using fundamental loop equations on the graph model was shown, which was experimentally validated on a two degrees of freedom planar manipulator. With this actuation Jacobian, stiffness and compliance can be mapped between actuation space and joint space. Stiffness control to achieve a desired stiffness was proposed, by minimizing a geometry based metric which compares stiffness matrices in joint space so that no non-intrinsic transformations, using the inverse of Jacobians, are necessary. This control is an optimization of the actuator topology and their stiffnesses, and defines design guidelines for compliant manipulators. Although design complexity and the fact that stiffness is configuration dependent are not taken into account in the proposed metric or optimization procedure, a simulation with a two degrees of freedom planar manipulator showed the effectiveness of this metric in optimizing stiffnesses under an end effector trajectory constraint. 


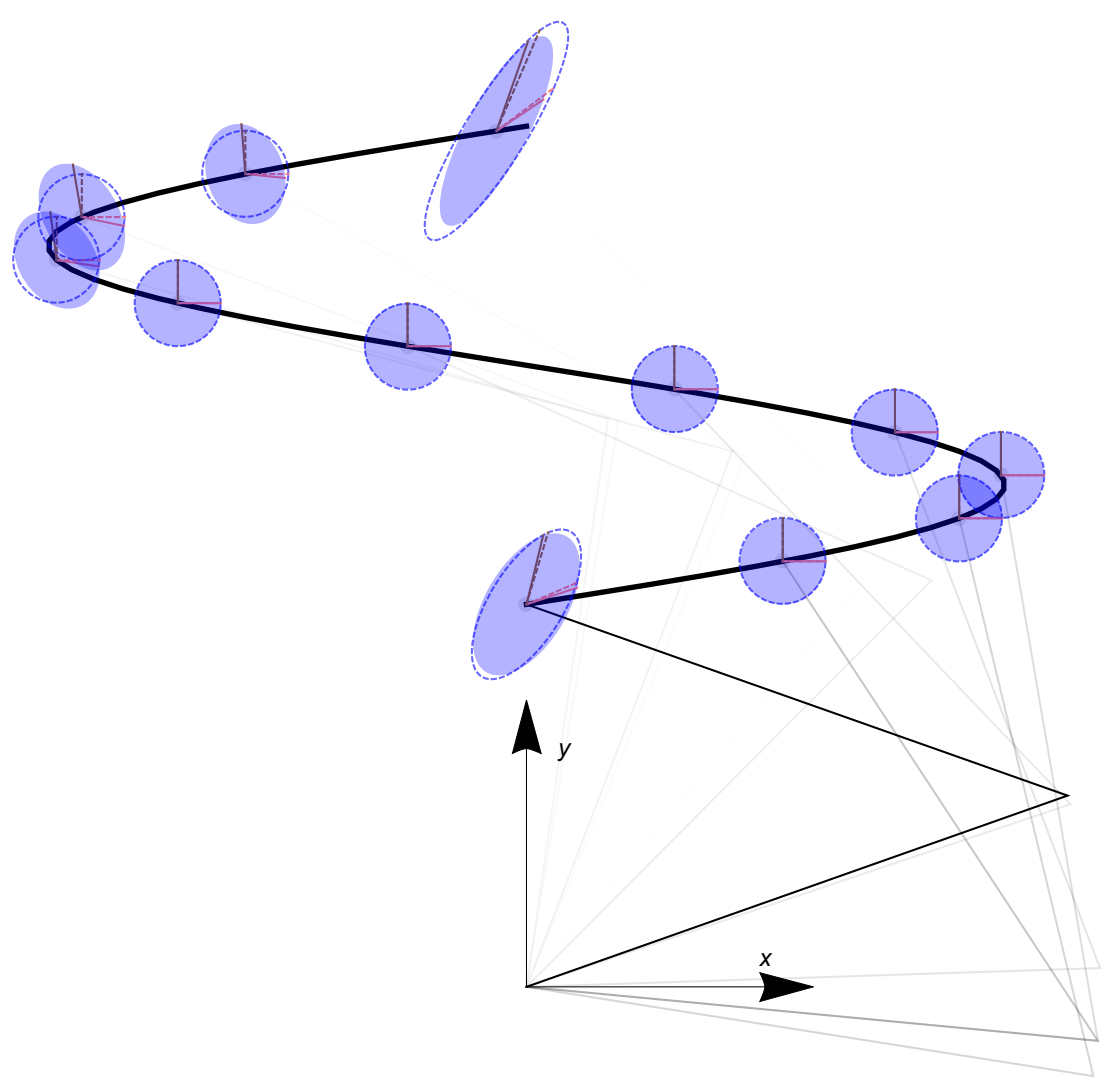

(a) Uniarticulation on the first and second joint, and biarticulation.

Figure 8.11: Stiffness control during a sinusoidal end effector trajectory. The best approximation is achieved when using all three possible actuators (twice uniarticulation, and biarticulation), while the worst situation is achieved when using only twice uniarticulation and no biarticulation. 


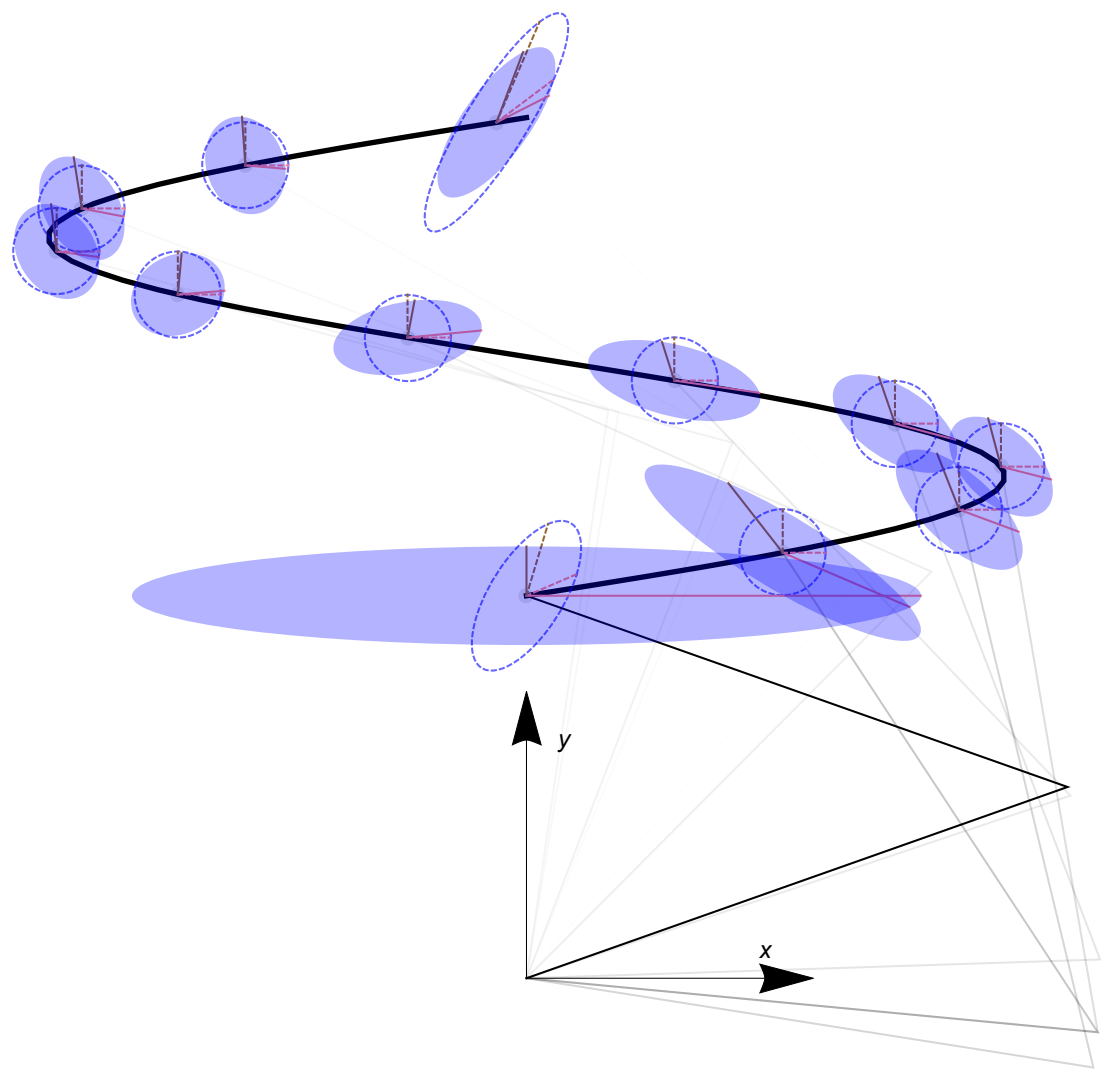

(b) Uniarticulation on the first joint, and biarticulation. 


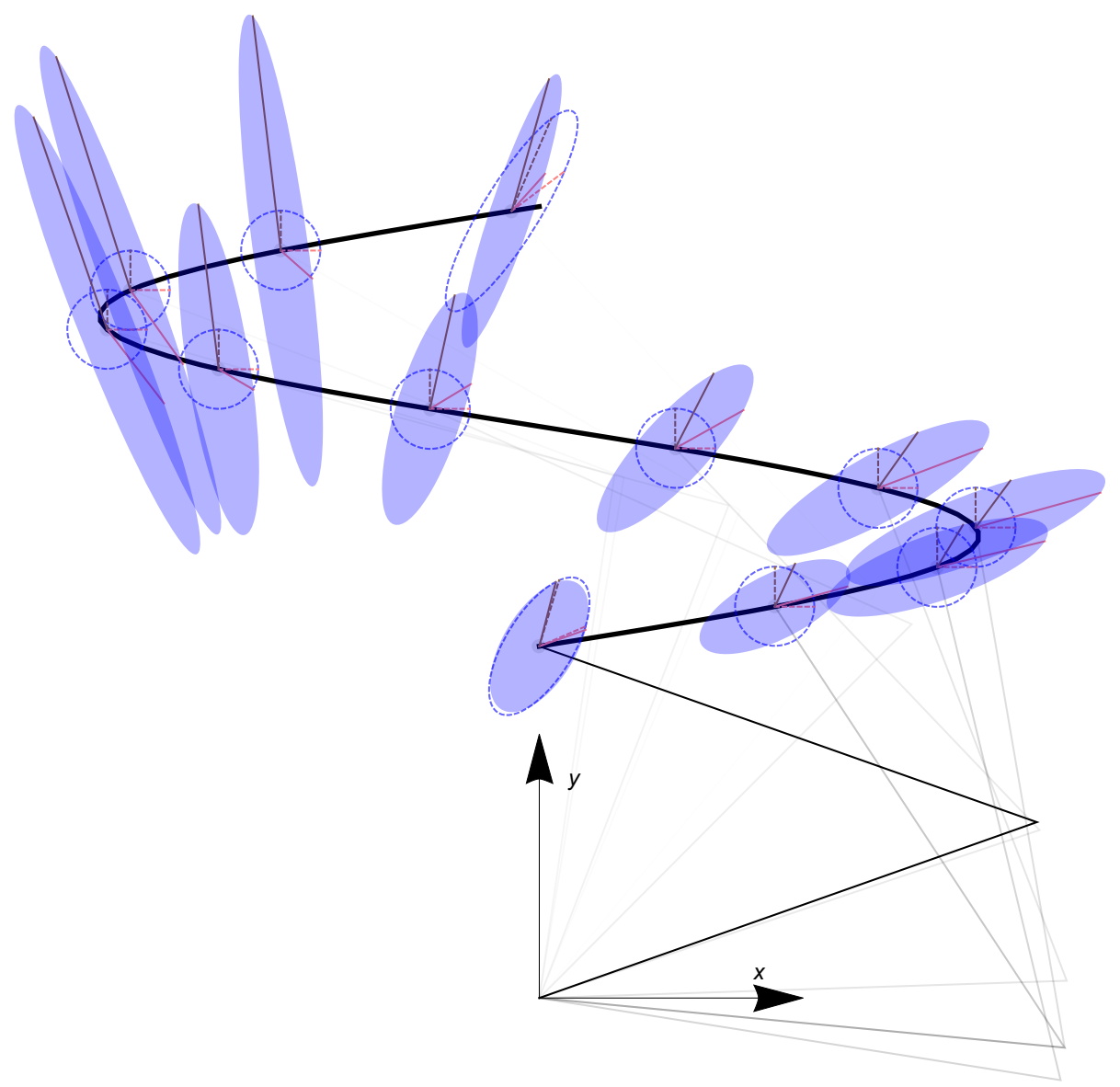

(c) Uniarticulation on the second joint, and biarticulation. 


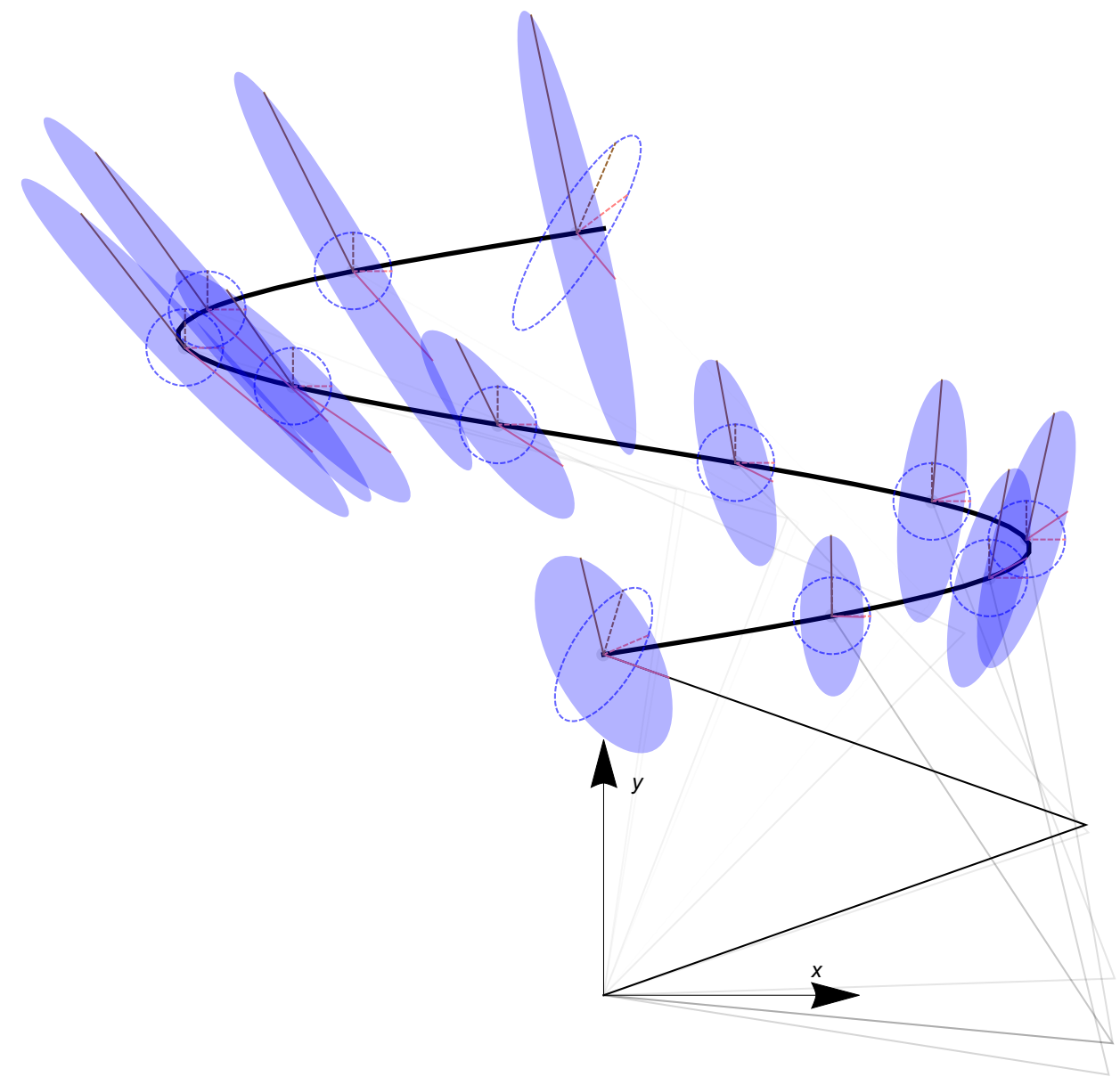

(d) Only uniarticulation on the first and second joint. 


\section{CHAPTER 9}

\section{Conclusions}

This thesis described the research on the modeling, design, and control of compliant robotic manipulators, set in the context of assistive robotic devices.

It was argued in Chapter 2 that variable stiffness joints offer great benefits for assistive robotic devices. Current systems have been evaluated on five usage criteria, that are based on usage surveys. These surveys indicate that eating and drinking, and picking up and manipulating objects are high priority tasks. Such tasks demand a high level of interaction between a user and the device. The properties of current assistive robotic platforms were qualitatively rated towards the proposed criteria, and it was argued that only the systems with variable stiffness joints could ensure a suitable rating for all criteria. However, as a consequence, the overall complexity of such systems increases which may lead to a higher risk of failure or malfunction, possibly due to unforeseen circumstances. Although the focus was placed on assistive robotic devices, all robotic devices that are used to interact with humans or need to operate in unstructured environments benefit from compliance, as achieved by using variable stiffness joints.

These variable stiffness joints can be realized in many different ways, as shown in Part I. Chapter 3 presents the variable stiffness actuator vsaUT-II. This actuator realizes a variable transmission ratio between the internal elastic elements' force, and the system output force, using a lever arm. A pivot that moves along the lever arm, by means of a planetary gear mechanism, determines this transmission ratio, which ranges from zero to infinite. The two degrees of freedom (position and stiffness change) are actuated by two separate motors. Since it was designed for an output torque of $60 \mathrm{Nm}$ and was properly manufactured, it is quite robust. The deflection is, however, quite small due to limited space, and end-stops, which means that little energy can be stored in the elastic elements. Chapter 4 presents the variable stiffness mechanism VSM, which is also based on a variable transmission ratio between the output and the elastic elements. However, the VSM does not use a lever arm with linear extension springs, but uses leaf springs. The VSM is, like the vsaUT-II, also capable of an infinite stiffness range by moving a pivot along the leaf springs. There is plenty of space for the deflection of these leaf springs, and it is also not limited by end-stops. Moreover, it can completely decouple the output from the input rotor, which means that an unlimited continuous passive output rotation is possible. An important feature of this mechanism is 
the ability to change the stiffness characteristic by shaping the leaf springs. Although the VSM is realized using rapid prototyping techniques which slightly affect the performance of the prototype, the benefits are apparent, which makes a highly engineered and properly manufactured version promising and feasible in robotic systems to be used for interaction with humans. The VSM also uses two motors to actuate the two degrees of freedom. In Chapter 5 a proof of concept of a variable stiffness actuator was presented which uses three actuators, of which two are small and low power solenoids, and one is a relatively high power motor. Therefore, this actuator is named SM-VSA, single motor variable stiffness actuator. It is able to actuate the output position and stiffness by locking and unlocking degrees of freedom in the actuator. This is done by bistable friction belt mechanisms, in which a friction belt can engage to or disengage from a surrounding cylinder, which causes it to, respectively, lock or unlock a degree of freedom. A prototype and an experiment validated the proof of concept. Although the prototype showed that the system is functional for limited operating conditions, it is in principle a considerably more complex design than the previous systems. The performance of a more optimized version should be further assessed.

It was argued that variable stiffness actuators should be the basis of assistive robotic devices, and, in particular, manipulators. These manipulators may have various kinematic structures, e.g., joint types and configurations, and link lengths, as well as many possibilities for the actuator placement, and articulation type. It might well be, for instance, that for a certain task or environment, a variable joint compliance is superfluous, and that constant compliance is more suitable. Hence, the design space for manipulators with many degrees of freedom becomes very large, and a tool to be able to model and analyze these systems is convenient. The tool that was used in Part II is graph theory. In Chapter 6, the graph theoretic one-dimensional models of a series elastic actuator, a variable stiffness actuator and a compliant system with multiple variable stiffness actuators have been presented, based on the Euler-Lagrange formalism. This showed the modularity of this approach to enlarge systems to multiple degrees of freedom in a straightforward way. A controller was applied to optimally tune the stiffnesses for various tasks, which showed that indeed an actuator need not necessarily be a variable stiffness actuator. However, the dynamics of these one-dimensional models are not representative for multi-body spatial dynamics, and, therefore, these results are of limited value. In Chapter 7, the model of generic six-dimensional compliant manipulators based on graph theory and the port-Hamiltonian framework was presented. It was shown that a manipulator's topology is decoupled from its dynamics, which allows a straightforward change of the actuators' connection by changing elements in an incidence matrix. This means that modeling multiarticulation is as easy as modeling uniarticulation, or any other articulation type. Chapter 8 treats the modeling and analysis of a compliant manipulator's stiffness. Since these graph models allow any articulation type, the actuators are not necessarily coupled to only one joint, making the actuation Jacobian, which maps the joint configuration velocities to actuator velocities, a diagonal matrix map. In case of multiarticulation, the Jacobian is not a diagonal matrix, and a structured approach to obtain it is provided using the graph models. Moreover, the control of actuator stiffnesses to obtain a desired work space stiffness is proposed. A metric weighs the geometric difference between the desired joint space stiffness and the achieved joint space stiffness. Minimizing this metric for the individual actuator stiffnesses, results in the optimal stiffness distribution for a specific configuration and kinematic structure. This was shown in simulation for a planar two degrees of freedom compliant manipulator. It was also shown that these results show very different performances for the various possible actuator connection topologies. One may use these results as design guidelines, to decide for an actuator topology with an acceptable approximation to the desired stiffness. Moreover, the configuration dependent stiffness in case of kinematically redundant manipulators is not 
taken into account, while this has a considerable effect on the end effector stiffness. Future research may investigate a combined metric which may propose a deviation of the desired configuration to closely achieve a desired stiffness.

In general, this research would have benefited from experiments with a robotic arm with multiple degrees of freedom and the possibility to relocate actuators easily to test the presence of multiarticulation. However, this arm could not be realized due to budget constraints, which meant making due with rapid prototyping small scale systems. Continuation of this research should focus on validating the presented concepts on full scale systems. Afterwards, one may pursue prospective user evaluations. 


\section{Appendix Preface}

These appendices summarize material and knowledge that was acquired over the course of the project. It is written to be a quick reference, and is in no way written to be the definitive resource and starting point for these subjects. The decision to trade-off intuition and mathematical (notational) precision has been made in favor of intuition. 


\section{APPENDIXA}

\section{Differential Geometry and Screw Theory}

This chapter treats some elementary concepts from differential geometry and screw theory. Further reading can be found in [130, 131] and [107].

\section{A.1 Manifolds}

A manifold is a topological space that is locally diffeomorphic to a Euclidean space, but not (necessarily) globally. The Earth's surface is locally flat, but globally certainly not. A general manifold can be visualized as a smooth blanket, to which one can zoom in to view the local Euclidean space. A Riemannian manifold is a manifold that has an inner product structure on the tangent space at each point. This inner product also induces a norm on this inner product space, i.e., the norm is the square root of the inner product of an element with itself. A Riemannian metric (a tensor field) makes it possible to define various geometric notions on a Riemannian manifolds, such as angles, lengths of curves, areas (or volumes), curvature, gradients of functions and divergence of vector fields.

\section{A.2 Groups}

A group $\mathscr{G}$ is a set $\mathscr{M}$ together with a binary operation $\bullet$, that takes two elements of the set and returns another, with identity element $I$ and three properties, namely associativity, invertability, and closure.

Given an operation $\bullet$, the associativity property holds:

$$
m_{1}, m_{2}, m_{3} \in \mathscr{M},\left(m_{1} \bullet m_{2}\right) \bullet m_{3}=m_{1} \bullet\left(m_{2} \bullet m_{3}\right) .
$$

The invertibility property is the following:

$$
\forall m \in \mathscr{M}, \exists m^{-1} \in \mathscr{M} \text { s.t. } m \bullet m^{-1}=I .
$$

The binary operation • implies set closure, i.e., taking two elements of the group and applying the binary operation implies that the resultant is again an element of the group: 
$m_{1} \bullet m_{2} \rightarrow m_{3}$ for $m_{1}, m_{2}, m_{3} \in \mathscr{M}$. If the binary operation does not return an element of the set $\mathscr{M}, \mathscr{G}$ is not a group.

Note that the binary operation is not necessarily commutative, i.e., the order of taking the binary operation matters. A commutative group is a group of which the binary operation is commutative, and is called an Abelian group.

Suppose, as an example, the integer numbers under multiplication. This does not form a group, since the invertibility property is not satisfied (the element zero has no inverse that, combined with zero in the binary operator, will result in the group's identity element). The integer numbers, except zero, under multiplication do form a group.

A finite group has a finite number of elements. An example is the symmetric group $S n$, where $n$ is the number of permutations (rearrangements of the set elements). Note these permutations are not a property of some operator, but it is the structure of the group itself.

An infinite group has an infinite number of elements. For example the group of positive real numbers $[\mathbb{R},+]$.

Examples:

- Integers under addition form a group;

- Integers under multiplication are not a group, since not every element has an inverse within the set (e.g. the element 0). This (a group in which not every element has an inverse) is then called a monoid;

- Integers without the zero element under multiplication are a group, since it satisfies the group properties.

\section{A.3 The Lie Group $S E(3)$}

The Lie group $S E(3)$ is the Special Euclidean group in three dimensions, and is a manifold group. This means that it is a group, and it is, moreover, smooth. Because the Lie group is smooth, differential calculus can be performed, which means that the derivative in space at a certain element of the group can be taken. The Special Euclidean group is an action group on a Euclidean space: elements of $S E(3)$ perform an action on points in the Euclidean space. This action is an isometric transformation of points to other points in the same Euclidean space, without the possibility of reflection. Very informally stated, one can think of the action on points as being a car that moves (action) the people inside (points). The group is Special since it is a subgroup (a special case) representing only isometries, i.e. rigid motions like translation and rotation of rigid bodies.

\section{A.4 Homogeneous Coordinates and Transformations}

A point in three dimensional space is an element and can be parameterized, or represented, by coordinates if a coordinate frame $\Psi$ is defined. A Cartesian coordinate frame assigns unique coordinates to points in space by orthogonal projection of the points on three mutually perpendicular axes (often indicated with the $x, y$ and $z$ ). In other words, the Cartesian coordinate frame defines a base for the representation of all points in space. Another representation can be found by using, for instance, polar coordinates. A point $p$ can be represented in a Cartesian 
coordinate frame $\Psi^{i}$ by:

$$
\bar{p}^{i}=\left[\begin{array}{l}
x \\
y \\
z
\end{array}\right] \in \mathbb{R}^{3} .
$$

Representing the same point in a different Cartesian coordinate frame $\Psi^{j}$ yields different numerical coordinates $x, y$ and $z$. The Lie group $S E(3)$ can represent a robot's posture in three dimensional space by associating coordinate frames to elements of the robot, and representing the relative configuration between those frames and a reference frame with $4 \times 4$ homogeneous matrices $H_{\bullet}^{\bullet} \in S E(3)$. A homogeneous matrix $H_{i}^{j}$ denotes the configuration of frame $i$ expressed in frame $j$ and has the following form:

$$
H_{i}^{j}=\left[\begin{array}{cc}
R_{i}^{j} & o_{i}^{j} \\
0 & 1
\end{array}\right]
$$

where $R_{i}^{j} \in S O(3)$ is a rotation matrix of the Special Orthonormal group in three dimensions, with the property that $\left(R_{i}^{j}\right)^{T}=\left(R_{i}^{j}\right)^{-1}$. The group is orthonormal since it refers to the orthonormal axes of the coordinate frames, i.e. mutually perpendicular and of length 1 , and the group is special because $\operatorname{det}\left(R_{i}^{j}\right)=1$. Moreover, $o_{i}^{j} \in \mathbb{R}^{3}$ is the origin of frame $\Psi^{i}$ expressed in frame $\Psi^{j}$. Note that $H_{i}^{j}=\left(H_{j}^{i}\right)^{-1}$.

To be able to easily change the coordinate representation of a point from one coordinate frame to another, i.e. a coordinate transformation, it is convenient to use homogeneous coordinates as representation of a point. The point $p$ represented by homogeneous coordinates in Cartesian frame $\Psi^{i}$ is:

$$
p^{i}=\left[\begin{array}{l}
x \\
y \\
z \\
1
\end{array}\right] \in \mathbb{R}^{4} .
$$

The coordinate change of a point represented in frame $\Psi^{i}, p^{i}$, to the same point represented in frame $\Psi^{j}, p^{j}$, is then given by a simple matrix multiplication, i.e.:

$$
p^{j}=H_{i}^{j} p^{i} .
$$

\section{A.5 Elements of the Tangent Spaces}

As was noted, the Lie group $S E(3)$ is a smooth manifold group, which has a differential structure. When defining smooth curves on the manifold, which represent isometric motions, the velocity at a certain configuration, i.e., group element, $H_{\bullet}^{\bullet} \in S E(3)$, can be considered. This velocity is tangent to the curve. Taking the derivative of a curve at $H_{\bullet}^{\bullet}$ yields an element $\dot{H}_{\bullet}^{\bullet} \in T_{H}: S E(3)$, i.e., an element that lies in the tangent plane $T$ (which is a vector space and, therefore, equivalently called the tangent space) at group element $H_{\bullet}^{\bullet}$ on the manifold $S E(3)$. The element $\dot{H}_{i}^{j}$ is a bounded vector at point $H_{i}^{j}$ on the manifold, and represents the relative change of configuration of frame $\Psi^{i}$ expressed in frame $\Psi^{j}$.

\section{A.6 Elements of the Algebras}

The tangent space at the identity $T_{I} S E(3)$ is a special tangent space (it has a skew-, or antisymmetric operation) and is called the Lie algebra, denoted by se(3). An element of the Lie 
algebra $s e(3)$ is called a twist $\tilde{T}_{\bullet}^{\bullet \bullet \bullet}$, and is, just like the group elements, a $4 \times 4$ matrix. Because of the group structure and the group identity property, it holds that $H_{i}^{j} H_{j}^{i}=H_{j}^{i} H_{i}^{j}=I$. An arbitrary group element can be mapped to the group identity element in two different ways, namely by left or right transport, or equivalently, by pre- or post-multiplication. It follows that also elements of the tangent spaces can be mapped to the tangent space in the identity, i.e. $\operatorname{se}(3)$.

$$
\begin{gathered}
\tilde{T}_{i}^{j, j}=\dot{H}_{i}^{j} H_{j}^{i} \in \operatorname{se}(3) \quad \text { (right) } \\
\tilde{T}_{i}^{i, j}=H_{j}^{i} \dot{H}_{i}^{j} \in \operatorname{se}(3) \quad \text { (left) }
\end{gathered}
$$

where twist $\tilde{T}_{i}^{k, j}$ is the instantaneous twist of frame $\Psi^{i}$ with respect to frame $\Psi^{j}$ expressed with coordinates in frame $\Psi^{k}$. These twists define infinitesimal or instantaneous motions independent of the (initial) configuration, since these twists are associated to the identity element $I \in S E(3)$, instead of to some $H_{\bullet}^{\bullet} \in S E(3)$. It can be shown that an arbitrary twist, for instance $\tilde{T}_{i}^{j, j}$, can be expressed in an arbitrary coordinate frame by:

$$
\tilde{T}_{i}^{k, j}=A d_{H_{j}^{k}} \tilde{T}_{i}^{j, j},
$$

where it can be shown that $A d_{H_{j}^{k}}$ has the following form:

$$
A d_{H_{j}^{k}}=\left[\begin{array}{cc}
R_{j}^{k} & 0 \\
\tilde{o}_{j}^{k} R_{j}^{k} & R_{j}^{k}
\end{array}\right],
$$

and the $4 \times 4$ twist is given by:

$$
\tilde{T}_{i}^{k, j}=\left[\begin{array}{cc}
\tilde{\omega}_{i}^{k, j} & v_{i}^{k, j} \\
0 & 0
\end{array}\right] .
$$

The element $\tilde{\omega}_{i}^{k, j} \in s o(3)$ is an element of the algebra $s o(3)$ on the Special Orthonormal group $S O(3)$ and is skew-symmetric and represents the rotational velocity of frame $\Psi^{i}$ with respect to frame $\Psi^{j}$ expressed in frame $\Psi^{k}$. Velocity $v_{i}^{k, j} \in \mathbb{R}^{3}$ is the velocity of a (possibly imaginary) point fixed in frame $\Psi^{i}$, with respect to $\Psi^{j}$, going through the origin of frame $\Psi^{k}$ and observed by an observer in $\Psi^{k}$. Twists as elements of se(3) are $4 \times 4$ matrices, with only six independent values. A parameterization of the twist $\tilde{T}$ is the 6-dimensional twist vector defined by the operator $\vee[131]$ as:

$$
\vee: \tilde{T} \rightarrow T:=\left[\omega_{x}, \omega_{y}, \omega_{z}, v_{x}, v_{y}, v_{z}\right]^{T} .
$$

A basis that can be used to represents twists, is given by the canonical basis twists:

$$
\begin{aligned}
& L_{1}=\left[\begin{array}{cccc}
0 & 0 & 0 & 0 \\
0 & 0 & -1 & 0 \\
0 & 1 & 0 & 0 \\
0 & 0 & 0 & 0
\end{array}\right], L_{2}=\left[\begin{array}{cccc}
0 & 0 & 1 & 0 \\
0 & 0 & 0 & 0 \\
-1 & 0 & 0 & 0 \\
0 & 0 & 0 & 0
\end{array}\right], L_{3}=\left[\begin{array}{cccc}
0 & -1 & 0 & 0 \\
1 & 0 & 0 & 0 \\
0 & 0 & 0 & 0 \\
0 & 0 & 0 & 0
\end{array}\right] \\
& L_{4}=\left[\begin{array}{llll}
0 & 0 & 0 & 1 \\
0 & 0 & 0 & 0 \\
0 & 0 & 0 & 0 \\
0 & 0 & 0 & 0
\end{array}\right], L_{5}=\left[\begin{array}{llll}
0 & 0 & 0 & 0 \\
0 & 0 & 0 & 1 \\
0 & 0 & 0 & 0 \\
0 & 0 & 0 & 0
\end{array}\right], L_{6}=\left[\begin{array}{llll}
0 & 0 & 0 & 0 \\
0 & 0 & 0 & 0 \\
0 & 0 & 0 & 1 \\
0 & 0 & 0 & 0
\end{array}\right]
\end{aligned}
$$

Every twist is then the linear combination of these basis twists. 
Wrenches are elements of the dual of the tangent space at the identity, so they are also $4 \times 4$ matrices. Again, a parameterization of the wrench $\tilde{W}$ is found by the operator $\vee$, which extracts the 6-dimensional co-vector which parameterizes the wrench by:

$$
\vee: \tilde{W} \rightarrow W:=\left[\tau_{x}, \tau_{y}, \tau_{z}, f_{x}, f_{y}, f_{z}\right]
$$

These wrenches are dual in the sense that the product of a wrench and a twist is a scalar representing the power that the wrench has transferred, i.e., $P=W T$.

\section{A.7 Lie Bracket}

The Lie bracket $[a, b]$ is a product operation between two elements $a$ and $b$ of the Lie algebra and it is defined as:

$$
[a, b]:=a b-b a .
$$

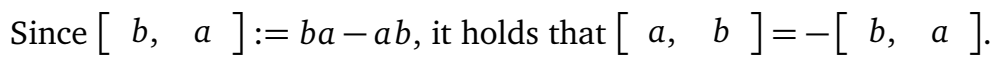

When applying the Lie bracket to $L_{1}$ and $L_{2}$ from Equation (A.12), it can be found that the third independent rotation is obtained:

$$
\begin{aligned}
{\left[\begin{array}{ll}
L_{1}, & L_{2}
\end{array}\right] } & =\left[\begin{array}{cccc}
0 & 0 & 0 & 0 \\
0 & 0 & -1 & 0 \\
0 & 1 & 0 & 0 \\
0 & 0 & 0 & 0
\end{array}\right]\left[\begin{array}{cccc}
0 & 0 & 1 & 0 \\
0 & 0 & 0 & 0 \\
-1 & 0 & 0 & 0 \\
0 & 0 & 0 & 0
\end{array}\right]-\left[\begin{array}{cccc}
0 & 0 & 1 & 0 \\
0 & 0 & 0 & 0 \\
-1 & 0 & 0 & 0 \\
0 & 0 & 0 & 0
\end{array}\right]\left[\begin{array}{cccc}
0 & 0 & 0 & 0 \\
0 & 0 & -1 & 0 \\
0 & 1 & 0 & 0 \\
0 & 0 & 0 & 0
\end{array}\right] \\
& =\left[\begin{array}{cccc}
0 & 0 & 0 & 0 \\
1 & 0 & 0 & 0 \\
0 & 0 & 0 & 0 \\
0 & 0 & 0 & 0
\end{array}\right]-\left[\begin{array}{llll}
0 & 1 & 0 & 0 \\
0 & 0 & 0 & 0 \\
0 & 0 & 0 & 0 \\
0 & 0 & 0 & 0
\end{array}\right] \\
& =\left[\begin{array}{cccc}
0 & -1 & 0 & 0 \\
1 & 0 & 0 & 0 \\
0 & 0 & 0 & 0 \\
0 & 0 & 0 & 0
\end{array}\right]=L_{3} .
\end{aligned}
$$

The Lie bracket is a special case of a commutator in groups, i.e., it is the infinitesimal version of the group commutator [132].

\section{A.8 Exponential Coordinates}

It follows that elements of the Lie algebra se(3) can be mapped to elements of the group $S E(3)$ by the map:

$$
H=e^{\tilde{\phi}} .
$$

The variable $\phi$ represents the finite twist, which geometrically belongs to $s e(3)$, but is different from the infinitesimal or instantaneous twist $\tilde{T}$. The finite twist is the motion that is performed if the twist is applied for exactly 1 second. Therefore, the finite twist $\tilde{\phi}$ is a configuration and has dimensions [rad] and [m], while the infinitesimal twist is a generalized velocity and has dimensions $[\mathrm{rad} / \mathrm{s}]$ and $[\mathrm{m} / \mathrm{s}][107]$. 


\section{A.9 Screws}

\section{A.9.1 Geometric interpretation of twists}

According to Chasles' theorem, any twist as represented in (A.11) can be represented by a screw motion, that is a rotation around an axis combined with a translation along that axis:

$$
\left[\begin{array}{l}
\omega \\
v
\end{array}\right]=\left[\begin{array}{c}
\omega \\
r \times \omega
\end{array}\right]+\lambda\left[\begin{array}{c}
0 \\
\omega
\end{array}\right]
$$

where $r$ is the position vector from the body frame origin to any point on the rotation axis, $x$ denotes the vector product and $\lambda$ is the pitch of the screw. The position vector $r$ and the pitch $\lambda$ can be defined by:

$$
\lambda:=\frac{\omega^{T} v}{\|\omega\|^{2}} \quad r=\frac{\omega \times v}{\|\omega\|^{2}} .
$$

\section{A.9.2 Geometric interpretation of wrenches}

An analogous reasoning for wrenches is defined by Poinsot's theorem: any system of forces can be represented by a torque around an axis combined with a translational force along that axis:

$$
\left[\begin{array}{l}
\tau \\
f
\end{array}\right]=\left[\begin{array}{c}
r \times f \\
f
\end{array}\right]+\lambda\left[\begin{array}{l}
f \\
0
\end{array}\right]
$$

Now, the screw pitch and position vector become:

$$
\lambda:=\frac{f^{T} \tau}{\|f\|^{2}} \quad r=\frac{f \times \tau}{\|f\|^{2}} .
$$




\section{APPENDIX B}

Port-Based Modeling

This chapter presents material on port-based modeling.

\section{B.1 Hamiltonian Systems}

Further reading on Hamiltonian systems and modeling of robotic systems, can be found in $[133,131]$ and [107].

\section{B.1.1 Hamiltonian equations}

For a conservative, autonomous, mechanical system, the canonical Hamiltonian equations are given by:

$$
\begin{aligned}
& \dot{q}=\frac{\partial H(q, p)}{\partial p} \\
& \dot{p}=-\frac{\partial H(q, p)}{\partial q} .
\end{aligned}
$$

This system is characterized by an energy function $H(q, p)$, called the Hamiltonian. This Hamiltonian is a function of the energy variables, i.e., $i$ generalized configuration coordinates $q=\left[q_{1}, \ldots, q_{i}\right]^{T}$ and $i$ generalized momentum coordinates $p=\left[p_{1}, \ldots, p_{i}\right]^{T}$, once local coordinates have been chosen. In mechanical systems, the Hamiltonian is the sum of kinetic and potential energy. A power balance can be derived using the energy function, i.e.,

$$
\frac{d}{d t} H(q, p)=\frac{\partial^{\mathrm{T}} H(q, p)}{\partial q} \frac{d q}{d t}+\frac{\partial^{\mathrm{T}} H(q, p)}{\partial p} \frac{d p}{d t}=0,
$$

expressing that the stored energy $H(q, p)$ is constant. Note that indeed the dimensions of $q$ and $p$ should be equal, i.e., their sum should always be even, for this power balance to be valid. 
When an external force is applied with which work can be done on the system, the Hamiltonian equations become:

$$
\begin{aligned}
& \dot{q}=\frac{\partial H(q, p)}{\partial p} \\
& \dot{p}=-\frac{\partial H(q, p)}{\partial q}+f \\
& e=\frac{\partial H(q, p)}{\partial p}
\end{aligned}
$$

where $f$ is the vector of generalized external forces, and $e$ is the dual variable representing the configuration changes. A similar power balance can be derived, i.e.,

$$
\frac{d}{d t} H(q, p)=\frac{\partial^{\mathrm{T}} H(q, p)}{\partial q} \frac{d q}{d t}+\frac{\partial^{\mathrm{T}} H(q, p)}{\partial p} \frac{d p}{d t}=\frac{\partial^{\mathrm{T}} H(q, p)}{\partial p} f=e^{\mathrm{T}} f,
$$

expressing that the change in stored energy $H(q, p)$ equals the supplied work [134]. The time derivative of the energy variables of the system, i.e., the independent states or conserved quantities, are called the flow variables $f$. The partial derivatives of the Hamiltonian $H(q, p)$ with respect to the energy variables are called the effort variables $e$. Their product defined by $P:=e^{\mathrm{T}} f$ is power. ${ }^{1}$

Equation (B.3) may be written in matrix form by:

$$
\begin{aligned}
& \dot{x}=S^{-1} \frac{\partial H(x)}{\partial x}+G(x) f \\
& e=G^{\mathrm{T}}(x) \frac{\partial H(x)}{\partial x},
\end{aligned}
$$

where $x=(q, p), S^{-1}=\left[\begin{array}{cc}0 & I \\ -I & 0\end{array}\right]$ (with $I$ the identity matrix), and $G(x)=\left[\begin{array}{ll}0 & I\end{array}\right]^{\mathrm{T}}$. Like stated before, the dimension of $x$ should be even.

\section{B.1.2 Generic port-Hamiltonian system}

A port-Hamiltonian system is given by:

$$
\begin{aligned}
& \dot{x}=J(x) \frac{\partial H(x)}{\partial x}+g(x) u \\
& y=g^{T}(x) \frac{\partial H(x)}{\partial x},
\end{aligned}
$$

with Hamiltonian $H(x)$, skew-symmetric (Poisson tensor) structure matrix $J(x)=-J^{\mathrm{T}}(x)$, local coordinates $x$ on manifold $\mathscr{X}$, input matrix $g(x)$, and collocated port variables $u$ and $y$. Because of the skew-symmetry of $J(x)$, the power balance is:

$$
\begin{aligned}
\frac{d}{d t} H(x)=\frac{\partial^{\mathrm{T}} H(x)}{\partial x} \frac{d x}{d t} & =\frac{\partial^{\mathrm{T}} H(x)}{\partial x}\left(J(x) \frac{\partial H(x)}{\partial x}+g(x) u\right) \\
& =\frac{\partial^{\mathrm{T}} H(x)}{\partial x} J(x) \frac{\partial H(x)}{\partial x}+\frac{\partial^{\mathrm{T}} H(x)}{\partial x} g(x) u=y^{\mathrm{T}} u .
\end{aligned}
$$

Therefore, the dimension of $x$ is not necessarily even.

\footnotetext{
${ }^{1}$ Note that freedom of notation is abused since flows are vectors, and efforts, the dual of flows, are co-vectors. Therefore, co-vectors should be represented by row arrays, but here they are represented by column arrays instead, hence needing the transpositions.
} 


\section{B.1.3 Input-state-output port-Hamiltonian system}

A specific case of the system in (B.6) is obtained, if a distinction is made between explicit ports, i.e. storage, control, interaction, and dissipation ports, to which energy flows are associated. Such an input-state-output port-Hamiltonian system, with control and interaction port, is given by:

$$
\begin{aligned}
& \dot{x}=[J(x)-R(x)] \frac{\partial H(x)}{\partial x}+g(x) u+h(x) d \\
& y=g^{T}(x) \frac{\partial H(x)}{\partial x} \\
& z=h^{T}(x) \frac{\partial H(x)}{\partial x}
\end{aligned}
$$

where $x=\left[x_{1}, \ldots, x_{k}\right]^{\mathrm{T}}$ is the $k$-dimensional vector of elements of the state manifold $\mathscr{X}, J(x)$ is the $k \times k$ skew-symmetric structure matrix, i.e. $J(x)=-J^{\mathrm{T}}(x)$, defining the interconnection between efforts and flows, $R(x) \geq 0$ is the $k \times k$ symmetric dissipative structure matrix, $g(x)$ is the $k \times r$ input matrix, $u \in \mathbb{R}^{r}$ is the vector of the generalized control inputs, $h(x)$ is the $k \times w$ interaction matrix, $d \in \mathbb{R}^{w}$ is the vector of generalized interaction inputs, $y \in \mathbb{R}^{r}$ is the generalized control output vector collocated with control input $u$ and $z \in \mathbb{R}^{w}$ is the generalized interaction output vector collocated with interaction input $d$. Note that $y^{\mathrm{T}} u$ is the power flow through the control port and $z^{\mathrm{T}} d$ is the power flow through the interaction port. A powercontinuous Dirac structure $(\mathscr{D})$ connects the ports and defines the power flow between them, as given by $J(x)$.

Port-Hamiltonian systems can be represented graphically by bond graphs. A model is then synthesized by drawing relationships, i.e., half arrows, defining the positive direction (which is thus merely a convention instead of a physical effect) of the power flow between ports. A little more on this is given in Section B.2.

\section{B.1.4 Rigid bodies}

A rigid body is a collection of points with individual mass $\delta m$ where the relative distance between all points is fixed, as opposed to a flexible body where the points can move relative to one another.

The dynamical behavior of a rigid body when there is no potential energy to consider, can be described by using only its momentum, i.e. its configuration is not needed. Then, only kinetic energy needs to be considered. Potential energy is only dependent on the configuration of the system, which can be represented in a coordinate independent way rather than assigning the generalized $Q$ coordinates as, e.g., Euler angles for the configuration. In case that there is potential energy, e.g. to describe gravity, the total energy of a rigid body is the sum of kinetic and potential energy, and is given by:

$$
H\left(P_{i}^{k}, \phi_{i}^{0}\right)=\frac{1}{2} P_{i}^{k}\left(I_{i}^{k}\right)^{-1}\left(P_{i}^{k}\right)^{\mathrm{T}}+V\left(\phi_{i}^{0}\right) .
$$

The port-Hamiltonian equations of a rigid body are given by [107]:

$$
\begin{aligned}
{\left[\begin{array}{c}
\dot{\phi}_{i}^{0} \\
\left(\dot{P}_{i}^{k}\right)^{\mathrm{T}}
\end{array}\right] } & =\left[\begin{array}{cc}
0 & \kappa\left(\phi_{i}^{0}\right) \circ A d_{H_{k}^{0}} \\
-A d_{H_{k}^{0}}^{\mathrm{T}} \circ \kappa^{-\mathrm{T}}\left(\phi_{i}^{0}\right) & -\tilde{P}_{i}^{k} \wedge
\end{array}\right]\left[\begin{array}{c}
\frac{\partial H}{\partial \phi_{i}^{0}} \\
\frac{\partial H}{\partial P_{i}^{k}}
\end{array}\right]+\left[\begin{array}{c}
0 \\
I
\end{array}\right] W_{\bullet}^{k, i} \\
T_{i}^{k, 0} & =[0, I]\left[\begin{array}{c}
\frac{\partial H}{\partial \phi_{i}^{0}} \\
\frac{\partial H}{\partial P_{i}^{k}}
\end{array}\right],
\end{aligned}
$$


where $\phi_{i}^{0}$ is the finite twist, or the exponential coordinate of configuration $H_{i}^{0}$ (as opposed to the infinitesimal or instantaneous twist $T$ ) which is related to the homogeneous configuration by the exponential map

$$
H_{i}^{0}=e^{\tilde{\phi}_{i}^{0}}
$$

The finite twist can be thought of as a configuration change resulting from applying an instantaneous twist for one second. Therefore, the finite twist can be thought of as a configuration itself. The rigid body momentum of body $i$ expressed in frame $k$ is represented by $P_{i}^{k}$, the map from instantaneous twist to finite twist is represented by $\kappa^{-1}, A d_{H_{k}^{0}}$ represents the coordinate change from frame $k$ to the inertial frame 0 and $\tilde{P}_{i}^{k} \wedge$ represent the map from instantaneous twist to rate of change of momentum, i.e., the total wrench on body $i$ expressed in frame $k$. The partial derivates of the Hamiltonian $H$ are, just like before, the efforts and $T_{i}^{k, 0}$ is the instantaneous twist of body $i$ with respect to the inertial frame 0 , expressed in frame $k$.

Note the similarities between (B.8) and (B.10). In the following sections, the various elements are elaborated.

\section{Rigid Body Energy}

The rigid body energy is given by:

$$
H\left(P_{i}^{k}, \phi_{i}^{0}\right)=\frac{1}{2} P_{i}^{k}\left(I_{i}^{k}\right)^{-1}\left(P_{i}^{k}\right)^{\mathrm{T}}+V\left(\phi_{i}^{0}\right)
$$

The kinetic energy (first term) is a quadratic form on the momentum space using the inverse inertia tensor. The rigid body momentem $P_{i}^{k}$ is related to the inertia tensor and instantaneous twist by:

$$
\left(P_{i}^{k}\right)^{\mathrm{T}}=I_{i}^{k} T_{i}^{k, 0} \text {. }
$$

The inertia tensor of body $i$ expressed in frame $k$ is given by $I_{i}^{k}$. If frame $\Psi_{k}$ is chosen to be a body fixed frame, the inertia tensor is constant. Moreover, if frame $\Psi_{k}$ is chosen as the principle inertia frame fixed to the body, the inertia tensor is constant and furthermore diagonal. For a frame chosen that is not fixed with body $i$, the inertia tensor is not constant and varies with the configuration.

The potential energy (second term) is given by the potential energy function $V\left(\phi_{i}^{0}\right)$. The most common form of potential energy associated to a rigid body is the gravity potential and is described in the principal inertia frame by:

$$
V\left(\phi_{i}^{0}\right)=m g \phi_{i, z}^{0}
$$

where $m$ is the mass of body $i$ concentrated in the origin of its principal inertia frame, $g$ is the gravitational acceleration and $\phi_{i, z}^{0}$ is the translational z-coordinate (the vertical axis parallel to the gravity field) of body $i$ expressed in the inertial frame 0 .

\section{Momentum Map}

For a one degree of freedom moving mass, Newton's second law states that the net force on a mass equals the time derivative of its momentum:

$$
\dot{p}(t)=F(t) .
$$

Extending this to rigid bodies in 6-dimensions and dropping the explicit time-dependence notation yields:

$$
\left(\dot{P}_{i}^{0}\right)^{\mathrm{T}}=\left(W_{\bullet}^{0, i}\right)^{\mathrm{T}}
$$


which can be rewritten in coordinate frame $\Psi_{k}$, e.g. body coordinates, by:

$$
\begin{aligned}
& \left(\dot{P}_{i}^{0}\right)^{\mathrm{T}}=\left(W_{\bullet}^{0, i}\right)^{\mathrm{T}} \\
& \left(A d_{H_{0}^{k}}^{\mathrm{T}}\left(P_{i}^{k}\right)^{\mathrm{T}}\right)=\left(W_{\bullet}^{0, i}\right)^{\mathrm{T}} \\
& \dot{A d_{H_{0}^{k}}^{\mathrm{T}}}\left(P_{i}^{k}\right)^{\mathrm{T}}+A d_{H_{0}^{k}}^{\mathrm{T}}\left(\dot{P_{i}^{k}}\right)^{\mathrm{T}}=\left(W_{\bullet}^{0, i}\right)^{\mathrm{T}} \\
& \left(A d_{H_{0}^{k}} a d_{T_{0}^{0, k}}\right)^{\mathrm{T}}\left(P_{i}^{k}\right)^{\mathrm{T}}+A d_{H_{0}^{k}}^{\mathrm{T}}\left(P_{i}^{k}\right)^{\mathrm{T}}=\left(W_{\bullet}^{0, i}\right)^{\mathrm{T}} \\
& A d_{H_{k}^{0}}^{\mathrm{T}} a d_{T_{0}^{0, k}}^{\mathrm{T}} A d_{H_{0}^{k}}^{\mathrm{T}}\left(P_{i}^{k}\right)^{\mathrm{T}}+A d_{H_{k}^{0}}^{\mathrm{T}} A d_{H_{0}^{k}}^{\mathrm{T}}\left(\dot{P}_{i}^{k}\right)^{\mathrm{T}}=A d_{H_{k}^{0}}^{\mathrm{T}}\left(W_{\bullet}^{0, i}\right)^{\mathrm{T}} \\
& a d_{T_{0}^{k, k}}^{\mathrm{T}}\left(P_{i}^{k}\right)^{\mathrm{T}}+\left(\dot{P_{i}^{k}}\right)^{\mathrm{T}}=\left(W_{\bullet}^{k, i}\right)^{\mathrm{T}} \\
& \left(\dot{P_{i}^{k}}\right)^{\mathrm{T}}=-a d_{T_{0}^{k, k}}^{\mathrm{T}}\left(P_{i}^{k}\right)^{\mathrm{T}}+\left(W_{\bullet}^{k, i}\right)^{\mathrm{T}} \\
& \left(\dot{P_{i}^{k}}\right)^{\mathrm{T}}=a d_{T_{k}^{k, 0}}^{\mathrm{T}}\left(P_{i}^{k}\right)^{\mathrm{T}}+\left(W_{\bullet}^{k, i}\right)^{\mathrm{T}} .
\end{aligned}
$$

The additional term that appears (first term on the right hand side) accounts for the fictitious Coriolis and centrifugal forces, that are non-existent in inertial reference frames. This can be further expanded to yield the momentum map from instantaneous twist to the time derivative of the momentum:

$$
\begin{aligned}
& \left(\dot{P_{i}^{k}}\right)^{\mathrm{T}}=a d_{T_{k}^{k, 0}}^{\mathrm{T}}\left(P_{i}^{k}\right)^{\mathrm{T}}+\left(W_{\bullet}^{k, i}\right)^{\mathrm{T}} \\
& \left(\dot{P}_{i}^{k}\right)^{\mathrm{T}}=\left[\begin{array}{cc}
\tilde{\omega}_{k}^{k, 0} & 0 \\
\tilde{v}_{k}^{k, 0} & \tilde{\omega}_{k}^{k, 0}
\end{array}\right]^{\mathrm{T}}\left[\begin{array}{c}
\left(P_{\omega, i}^{k}\right)^{\mathrm{T}} \\
\left(P_{v, i}^{k}\right)^{\mathrm{T}}
\end{array}\right]+\left(W_{\bullet}^{k, i}\right)^{\mathrm{T}} \\
& \left(\dot{P_{i}^{k}}\right)^{\mathrm{T}}=\left[\begin{array}{cc}
\left(\tilde{\omega}_{k}^{k, 0}\right)^{\mathrm{T}} & \left(\tilde{v}_{k}^{k, 0}\right)^{\mathrm{T}} \\
0 & \left(\tilde{\omega}_{k}^{k, 0}\right)^{\mathrm{T}}
\end{array}\right]\left[\begin{array}{c}
\left(P_{\omega, i}^{k}\right)^{\mathrm{T}} \\
\left(P_{v, i}^{k}\right)^{\mathrm{T}}
\end{array}\right]+\left(W_{\bullet}^{k, i}\right)^{\mathrm{T}} \\
& \left(\dot{P}_{i}^{k}\right)^{\mathrm{T}}=\left[\begin{array}{c}
-\tilde{\omega}_{k}^{k, 0}\left(P_{\omega, i}^{k}\right)^{\mathrm{T}}-\tilde{v}_{k}^{k, 0}\left(P_{v, i}^{k}\right)^{\mathrm{T}} \\
-\tilde{\omega}_{k}^{k, 0}\left(P_{v, i}^{k}\right)^{\mathrm{T}}
\end{array}\right]+\left(W_{\bullet}^{k, i}\right)^{\mathrm{T}} \text { (because of skew-symmetry) } \\
& \left(\dot{P_{i}^{k}}\right)^{\mathrm{T}}=\left[\begin{array}{c}
\left(\tilde{P}_{\omega, i}^{k}\right)^{\mathrm{T}} \omega_{k}^{k, 0}+\left(\tilde{P}_{v, i}^{k}\right)^{\mathrm{T}} v_{k}^{k, 0} \\
\left(\tilde{P}_{v, i}^{k}\right)^{\mathrm{T}} \omega_{k}^{k, 0}
\end{array}\right]+\left(W_{\bullet}^{k, i}\right)^{\mathrm{T}} \text { (because of skew-symmetry) } \\
& \left(\dot{P}_{i}^{k}\right)^{\mathrm{T}}=\left[\begin{array}{cc}
\left(\tilde{P}^{k}{ }_{\omega, i}\right)^{\mathrm{T}} & \left(\tilde{P}_{v, i}^{k}\right)^{\mathrm{T}} \\
\left(\tilde{P}_{v, i}^{k}\right)^{\mathrm{T}} & 0
\end{array}\right]\left[\begin{array}{c}
\omega_{k}^{k, 0} \\
v_{k}^{k, 0}
\end{array}\right]+\left(W_{\bullet}^{k, i}\right)^{\mathrm{T}} \\
& \left(\dot{P}_{i}^{k}\right)^{\mathrm{T}}=\left[\begin{array}{cc}
-\tilde{P}_{\omega, i}^{k} & -\tilde{P}_{v, i}^{k} \\
-\tilde{P}_{v, i}^{k} & 0
\end{array}\right]\left[\begin{array}{c}
\omega_{k}^{k, 0} \\
v_{k}^{k, 0}
\end{array}\right]+\left(W_{\bullet}^{k, i}\right)^{\mathrm{T}} \\
& \left(\dot{P}_{i}^{k}\right)^{\mathrm{T}}=-\left[\begin{array}{cc}
\tilde{P}_{\omega, i}^{k} & \tilde{P}_{v, i}^{k} \\
\tilde{P}_{v, i}^{k} & 0
\end{array}\right]\left[\begin{array}{c}
\omega_{k}^{k, 0} \\
v_{k}^{k, 0}
\end{array}\right]+\left(W_{\bullet}^{k, i}\right)^{\mathrm{T}} \\
& \left(\dot{P}_{i}^{k}\right)^{\mathrm{T}}=-\tilde{P}_{i}^{k} T_{k}^{k, 0}+\left(W_{\bullet}^{k, i}\right)^{\mathrm{T}} \text {. }
\end{aligned}
$$

So, $\tilde{P}_{i}^{k}$ is a representation for the map $\tilde{P}_{i}^{k} \wedge: T_{k}^{k, 0} \mapsto\left(\dot{P}_{i}^{k}\right)^{\mathrm{T}}$. The result of (B.18) can in fact be found in (B.10).

\section{Twist map}

The map $\kappa$ in (B.10) defines the map between the finite and instantaneous twist [107], i.e.: 


$$
T_{i}^{0,0}=\kappa^{-1}\left(\phi_{i}^{0}\right) \dot{\phi}_{i}^{0} .
$$

By using the inverse mapping of (B.11), the mapping defined by $\kappa\left(\phi_{i}^{0}\right)$ can be found:

$$
\begin{aligned}
\frac{d}{d t} \tilde{\phi}_{i}^{0} & =\frac{d}{d t} \ln \left(H_{i}^{0}\right) \\
\dot{\phi}_{i}^{0} & =\left(e^{a d_{\phi_{i}^{0}}}-I\right)^{-1} a d_{\phi_{i}^{0}} T_{i}^{0,0} \\
\dot{\phi}_{i}^{0} & =\kappa\left(a d_{\phi_{i}^{0}}\right) T_{i}^{0,0} \\
\dot{\phi}_{i}^{0} & =\kappa\left(a d_{\phi_{i}^{0}}\right) \circ A d_{H_{k}^{0}} T_{i}^{k, 0} .
\end{aligned}
$$

By using the techniques presented in [135], this mapping can be calculated.

It is shown that any analytic, scalar funcion $f(x)$ with scalar $x$ can be extended to a square matrix function $F(A)$ with square matrix $A$ and any matrix function can be written as a polynomial matrix function according to the Cayley-Hamilton theorem. This also holds for the map $\kappa()$ :

$$
\begin{aligned}
& \kappa^{-1}\left(a d_{\phi_{i}^{0}}\right)=\left(e^{a d_{\phi_{i}^{0}}}-I\right)^{-1} a d_{\phi_{i}^{0}} \\
& \kappa^{-1}\left(a d_{\phi_{i}^{0}}\right)=c_{5} a d_{\phi_{i}^{0}}^{5}+c_{4} a d_{\phi_{i}^{0}}^{4}+c_{3} a d_{\phi_{i}^{0}}^{3}+c_{2} a d_{\phi_{i}^{0}}^{2}+c_{1} a d_{\phi_{i}^{0}}+c_{0} I .
\end{aligned}
$$

The powers of the square adjoint matrices can be calculated straightforwardly and the coefficients $c_{\bullet}$ were found above, which are:

$$
\begin{aligned}
& c_{0}=1 \\
& c_{1}=-\frac{1}{2} \\
& c_{2}=\frac{3 w \sin (w)+8 \cos (w)-8+w^{2}}{4 w^{2}(\cos (w)-1)} \\
& c_{3}=0 \\
& c_{4}=\frac{w \sin (w)+4 \cos (w)-4+w^{2}}{4 w^{4}(\cos (w)-1)} \\
& c_{5}=0 .
\end{aligned}
$$

\section{B.1.5 Kinematic pairs}

Kinematic pairs constrain the relative motion between rigid bodies. Kinematic constraints of a robotic manipulator are defined in its joints by, in general, holonomic constraints. Holonomic constraints are constraints that can be defined as being a function of only the state $q$ and time $t$, and not of the velocity $\dot{q}$. Constraints that cannot be expressed like this are non-holonomic.

Holonomic equality constraints can be given by functions of the form: $\varphi(q)=0$, where $q$ are properly chosen local coordinates for the joint under consideration. In case of a rotation joint allowing only local $y$ rotations, i.e., rotations in direction $q_{2}$, the constraint can be given as follows:

$$
\varphi(q)=\left[\begin{array}{l}
\varphi_{1}(q) \\
\varphi_{3}(q) \\
\varphi_{4}(q) \\
\varphi_{5}(q) \\
\varphi_{6}(q)
\end{array}\right]=\left[\begin{array}{l}
q_{1}=0 \\
q_{3}=0 \\
q_{4}=0 \\
q_{5}=0 \\
q_{6}=0
\end{array}\right]
$$


Taking the derivative results in velocity constraints that are linear in the velocity $\dot{q}$ (also called Pfaffian constraints):

$$
\dot{\varphi}(q)=\frac{\partial \varphi(q)}{\partial q} \dot{q}=\dot{q}_{c}=J_{c}(q) \dot{q}=0,
$$

and gives rise to the constraint Jacobian $J_{c}$. There can be no energy storage at a kinematic pair and the principle of virtual work holds, which says that no work should be done by any constraint force $\lambda$ through the constraint velocities $\dot{q}_{c}$.

The kinematic pairs described here, describe constraints on all local joint coordinates. Using such full-coordinate kinematic pairs has the advantage that one can see the reaction forces in a joint in the constraint directions (by the Lagrange multipliers $\lambda$ and constraint Jacobian $J_{c}(q)$ ), and that it is flexible with respect to changes. When using reduced coordinates, the constraint directions are just ignored and only the degrees of freedom are considered.

\section{B.2 Bond Graphs}

Bond graphs are a tool to model and visualize port-based models of physical systems (like the modeling presented in Section B.1). A bond is a directed half-arrow that represents positive power flow, where this power flow is the product of dual variables effort $e$ and flow $f$, i.e., $P:=e^{\mathrm{T}} f$. It makes use of various elements to represent energy supply (effort source Se, and flow source $\mathrm{Sf}$ ), energy dissipation $(\mathrm{R})$, and energy storage (C, accumulating flow, and I, accumulating effort). The junction structure couples these elements using common effort couplings (0-junction), common flow couplings (1-junction), and power-continuous energy converters (transformer TF, and gyrator GY).

In generalized bond graphs, the dual notion of C-type and I-type storage is removed, since one can be formed by the other by using an additional gyrator GY. The same holds for an effort and flow source. Generalized bond graphs follow naturally from the use of port-Hamiltonian models.

Further reading can be found in [107, 136, 137, 138] and [139]. 


\section{APPENDIX $\mathrm{C}$}

\section{Optimal Control}

The standard nonlinear optimal control problem can be considered as minimizing a certain cost functional under certain constraints. In particular, minimize:

$$
J=p(x(T), T)+\int_{0}^{T} q(x(t), u(t), t) \mathrm{d} t
$$

over horizon length $T$, where the cost $J \in \mathbb{R}$ is a function of the final cost $p(\cdot) \in \mathbb{R}$ and the running cost $q(\cdot) \in \mathbb{R}$, while the state $x(t)$ is constrained by the system dynamics:

$$
\dot{x}(t)=f(x(t), u(t)),
$$

and the control is possibly constraint to a set

$$
u(t) \in \mathscr{U} .
$$

For this problem, several approaches and corresponding methods can be considered, as depicted in $[140,141,89]$, and three of which are briefly summarized here.

\section{C.1 Dynamic Programming Approach}

The nonlinear optimal control problem may be transformed to a dynamic programming problem, which is based on Bellman's Optimality Principle, reducing the continuous infinite dimensional problem to a discrete finite dimensional problem. Bellman's Optimality Principle states that "An optimal policy has the property that whatever the initial state and initial decision are, the remaining decisions must constitute an optimal policy with regard to the state resulting from the first decision." [142]. The corresponding Hamilton-Jacobi-Bellman partial differential equation may be solved in reverse time to yield a solution for the value function, which can be thought of as the minimum cost-to-go from the current state. If such a (not necessarily unique) solution exists, the control input obtained is indeed optimal. Although this yields a state feedback control law and it is a global approach, since it is not dependent on specific initial conditions, it is in general difficult to obtain an accurate solution. 


\section{C.2 Variational Approach}

The nonlinear optimal control problem may also be approached using a variational approach, concerned with the minimization or maximization of functionals [143]. A possible approach is Pontryagin's Maximum Principle and the corresponding necessary optimality conditions. Using these conditions, differential equations in the state and co-state can be derived that define a boundary value problem (BVP, for initial and final constraints), or a multi-point boundary value problem (MPBVP, for trajectory tracking with additional internal and, therefore, continuity constraints), which can be solved using for instance gradient descent (which looks for the solution in the direction of the largest gradient) and multiple-shooting methods (which solves sections of the problem independently and imposes continuity constraints afterwards to retrieve a solution over the full interval). Although this approach can be realized quickly and is fairly straightforward to calculate, it yields an open loop solution for the control, i.e., it is only a function of time and not of the state. Moreover, it is not a global approach, since the solution is different for another initial condition.

\section{C.3 Iterative Approach}

The best of both approaches can be obtained when using iterative methods. Iterative methods are based on iteratively solving a sub-problem to improve the solutions for the state and control trajectories of the initial problem. The iterative strategies are local optimizations, since the nominal state and control trajectories are updated if at the current time step a new control yields a lower cost. Examples include differential dynamic programming [144, 145], receding-horizon control (or model-predictive control) [146] and iterative linear quadratic regulation (iLQR) [88]. Differential dynamic programming is based on Bellman's optimality principle and uses the notion of dynamic programming to define a finite dimensional problem. However, it approximates the cost quadratically around the current control and state and solves it recursively to obtain a nominal control. This nominal control dictates the nominal state trajectory, which are both the new trajectories around which the cost is approximated quadratically in the next iteration. This iterative scheme continues until convergence, which may only happen, possibly slowly, in case of smooth unconstrained problems [147]. Recedinghorizon control is based on Pontryagin's Maximum Principle. It uses the open-loop control solution of a finite horizon optimal control problem and applies that solution to the system for a fraction of the horizon length. After this fraction of time, again a finite horizon optimal control problem is solved which is, again, applied for a fraction of the horizon length, which results in a sampled feedback control. This scheme, however, may lack stability and its performance is highly dependent on the chosen finite horizon and the fraction of this horizon for which the solution is applied [140]. The iLQR method approximates the nonlinear system dynamics linearly and the cost function quadratically at each time step along the current state and control trajectories. This allows the method to solve an LQR problem to obtain a new control, which is accepted as an improved optimal control if the total cost has decreased. In that case, an improved state trajectory is obtained. The next iteration uses the new and improved state and control trajectories to, again, approximate the system dynamics linearly and cost function quadratically at each time step along these trajectories. The control is then further refined to improve the state and control trajectories and these iterations continue until the improvement of the cost has become sufficiently small. This method is used for the control of compliant actuators and manipulators in $[78,88,89]$, where it has been shown that it is well suited for this type of systems and is more efficient than comparative schemes. 


\section{APPENDIX D}

\section{VSM Patent}

During the project, a prototype of the novel variable stiffness mechanism VSM was developed. A patent application was done, of which the publication is provided here [63].

The invention relates to a coupling system for transmitting torque around a central axis of the coupling system, wherein the coupling system comprises; - a stator, - a hypocycloid transmission system rotatably supported by the stator, - an output member for transmitting torque, which output member is rotatably coupled with the stator through the hypocycloid transmission system, wherein, the hypocycloid transmission system comprises a planetary wheel member provided with a drive cam, the output member comprises a bending member at one end thereof coupled with the output member at an outer circumference thereof which bending member extends from the outer circumference of the output member towards the central axis, the drive cam contacts the bending member for driving the output member, and the radial position of the drive cam with respect to the bending member is adjustable through the hypocycloid transmission system, between an outer position proximate the circumference of the output member and an inner position proximate the central axis, for adjusting a desired stiffness of the coupling system. 
(12) INTERNATIONAL APPLICATION PUBLISHED UNDER THE PATENT COOPERATION TREATY (PCT) (19) World Intellectual Property Organization

International Bureau

(43) International Publication Date 9 July $2015(09.07 .2015)$

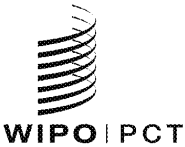

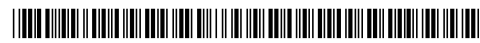

(10) International Publication Number

WO 2015/102490 A1

(51) International Patent Classification F16H 35/02 (2006.01)

(21) International Application Number:

PCT/NL2014/050919

(22) International Filing Date

30 December 2014 (30.12.2014)

(25) Filing Language:

English

(26) Publication Language:

English

(30) Priority Data:

61/921,654 30 December 2013 (30.12.2013)

$14165311.3 \quad 18$ April 2014 (18.04.2014)

US

EP

(71) Applicant: UNIVERSITEIT TWENTE [NL/NL]; Drienerlolaan 5, NL-7522 NB Enschede (NL).

(72) Inventors: STRAMIGIOLI, Stefano; Drienerlolaan 5, NL-7522 NB Enschede (NL). GROOTHUIS, Stefan Sebastiaan; Drienerlolaan 5, NL-7522 NB Enschede (NL).

(74) Agent: NEDERLANDSCH OCTROOIBUREAU; J.W Frisolaan 13, NL-2517 JS The Hague (NL).

(81) Designated States (unless otherwise indicated, for every kind of national protection available): $\mathrm{AE}, \mathrm{AG}, \mathrm{AL}, \mathrm{AM}$ $\mathrm{AO}, \mathrm{AT}, \mathrm{AU}, \mathrm{AZ}, \mathrm{BA}, \mathrm{BB}, \mathrm{BG}, \mathrm{BH}, \mathrm{BN}, \mathrm{BR}, \mathrm{BW}, \mathrm{BY}$, $\mathrm{BZ}, \mathrm{CA}, \mathrm{CH}, \mathrm{CL}, \mathrm{CN}, \mathrm{CO}, \mathrm{CR}, \mathrm{CU}, \mathrm{CZ}, \mathrm{DE}, \mathrm{DK}, \mathrm{DM}$, DO, DZ, EC, EE, EG, ES, FI, GB, GD, GE, GH, GM, GT, HN, HR, HU, ID, IL, IN, IR, IS, JP, KE, KG, KN, KP, KR KZ, LA, LC, LK, LR, LS, LU, LY, MA, MD, ME, MG, MK, MN, MW, MX, MY, MZ, NA, NG, NI, NO, NZ, OM, PA, PE, PG, PH, PL, PT, QA, RO, RS, RU, RW, SA, SC, SD, SE, SG, SK, SL, SM, ST, SV, SY, TH, TJ, TM, TN, TR, TT, TZ, UA, UG, US, UZ, VC, VN, ZA, ZM, ZW.

84) Designated States (unless otherwise indicated, for every kind of regional protection available): $\mathrm{ARIPO}(\mathrm{BW}, \mathrm{GH}$ GM, KE, LR, LS, MW, MZ, NA, RW, SD, SL, ST, SZ, TZ, UG, ZM, ZW), Eurasian (AM, AZ, BY, KG, KZ, RU TJ, TM), European (AL, AT, BE, BG, CH, CY, CZ, DE, DK, EE, ES, FI, FR, GB, GR, HR, HU, IE, IS, IT, LT, LU, LV, MC, MK, MT, NL, NO, PL, PT, RO, RS, SE, SI, SK, $\mathrm{SM}, \mathrm{TR})$, OAPI (BF, BJ, CF, CG, CI, CM, GA, GN, GQ, GW, KM, ML, MR, NE, SN, TD, TG).

Published:

- with international search report (Art. 21(3))

(57) Abstract: The invention relates to a coupling system for transmitting torque around a central axis of the coupling system, wherein the coupling system comprises; - a stator, - a hypocycloid transmission system rotatably supported by the stator, - an output member for transmitting torque, which output member is rotatably coupled with the stator through the hypocycloid transmission sys

tem, wherein, the hypocycloid transmission system comprises a planetary wheel member provided with a drive cam, the output mem-

- ing con the bend

ing member extends from the on

member for driving the output member, and the radial position of the drive cam with respect to the bending member is adjustable through the hypocycloid transmission system,between an outer position proximate the circumference of the output member and an inner position proximate the central axis, for adjusting a desired stiffness of the coupling system. 


\section{Variable stiffness actuator}

\section{Background}

The present invention relates to a coupling system for transmitting torque around a central axis of the coupling system, wherein the coupling system has an adjustable stiffness.

Such a coupling system is known from US 2013178297 which relates to a stiffness adjustable rotary joint.

10

\section{Summary of the invention}

The invention aims to provide a coupling system having an improved adjustment range.

15 Another object of the invention is to improve a coupling in that a problem associated therewith is at least partly solved.

Yet another object of the invention is to provide an alternative coupling system.

According to a first aspect of the invention this is realized with a coupling system for transmitting torque around a central axis of the coupling system, wherein the

20 coupling system comprises;

- a stator,

- a hypocycloid transmission system rotatably supported by the stator,

- an output member for transmitting torque, which output member is rotatably coupled with the stator through the hypocycloid transmission system,

25 wherein, the hypocycloid transmission system comprises a planetary wheel member provided with a drive cam, the output member comprises a bending member at one end thereof coupled with the output member at an outer circumference thereof which bending member extends from the outer circumference of the output member towards the central axis, the drive cam contacts the bending member for driving the output

30 member, and the radial position of the drive cam with respect to the bending member is adjustable through the hypocycloid transmission system, between an outer position proximate the circumference of the output member and an inner position proximate the central axis, for adjusting a desired stiffness of the coupling system. 
The planetary wheel member provided with the drive cam, and the bending member extending from the outer circumference of the output member towards the central axis enables to adjust the stiffness between a maximum stiffness to zero. In case of zero stiffness, the output member is uncoupled with respect to the hypocycloid

5 transmission system. This hypocycloid transmission system may also be referred to as a rotor.

The claimed system is named a coupling system since it intends to couple adjacent bodies like two links of a robot arm to form a joint between these links.

In an embodiment of the coupling system, the hypocycloid transmission system comprise a carrier supporting the planetary wheel member, and an annular member which engages the planetary wheel member at an outer circumference thereof in a driving manner, and wherein the radial position of the drive cam with respect to the bending member is adjustable through rotating the carrier with respect to the annular 15 member.

In an embodiment of the coupling system, the inner position of the drive cam coincides with the central axis such that the output member may rotate freely around the drive cam. This uncouples the output member from the rotor which may be desired 20 or beneficial in view of safety and/or efficiency in terms of use of energy.

In an embodiment of the coupling system, the hypocycloid transmission system comprises two planetary wheel members which mutually engage at the central axis in a driving manner. This is beneficial since in this way the coupling system is more

25 balanced and symmetrical.

The hypocycloid transmission system may be a planetary gear system. It is however conceivable that the hypocycloid system comprises a rolling link mechanism between planetary wheel members or between the annular member and a planetary

30 wheel member. Such a rolling link mechanism is known per se and stabilizes a rolling contact joint through one or more stabilization members like a band or flexure.

It is noted that when the hypocycloid transmission system is a planetary gear system, a sun gear is not really required for the functioning of the coupling system. 
In an embodiment of the coupling system the drive cam is integrally formed with the planetary wheel member. This simplifies the design of the coupling system and reduces the number of parts.

In an embodiment of the coupling system the drive cam is pivotable with respect to the planetary wheel member. This enables to provide the cam with opposite contact areas for driving the output member both clockwise and counter clockwise.

In an embodiment of the coupling system, the bending element extends diametrical with respect to the central axis and the hypocycloid transmission system. This enables to mount the bending element at both its opposite end on the annular member which limits deformation of the bending element and is beneficial in view of control of the coupling system and its stiffness. The bending element may be mounted

15 with the annular member in a sliding manner for accommodating deformation of the bending element.

In an embodiment, the coupling system comprises two bending elements

In an embodiment of the coupling system, the two bending elements constitute a slit between them for receiving the cam or cams.

In an embodiment of the coupling system, the slit extends diametrical with respect to the central axis and the hypocycloid transmission system.

25

In an embodiment of the coupling system two bending elements are integrally formed.

In an embodiment of the coupling system, the two planetary wheel members are each provided with a drive cam. This enables to drive the output member in a symmetrical way. 
In an embodiment of the coupling system, the drive cams are configured to mate in their inner position at the central axis such that the output member may rotate freely with respect to the hypocycloid transmission.

In an embodiment of the coupling system, the drive cams are configured to form a cylinder when they mate in their inner position at the central axis.

The invention further relates to a robotic system comprising a coupling system according to the invention. Such a robotic system may find use in care taking which

10 involves interaction with humans. In this connection "robotic system" may also refer to prostheses and so-called exoskeletons.

The invention further relates to a use of a coupling system according to the invention in a robotic system. drawings.

The invention further relates to a method comprising one or more of the characterising features described in the description and/or shown in the attached 20 drawings.

The various aspects discussed in this patent can be combined in order to provide additional advantages.

\section{Description of the drawings}

25

The invention will be further elucidated referring to a preferred embodiment shown in the schematic drawings wherein shown in:

Fig. 1 in perspective view an embodiment of the coupling system according to the invention;

30 fig. 2 a top view of the coupling system according to fig. 1;

fig 3 a schematic top view of the hypocycloid transmission system;

fig 4a-e a number of embodiments of the output member;

fig $5 \mathrm{a}$ an embodiment of the coupling system having one planetary member; 
5

fig $5 \mathrm{~b}$ an embodiment of the coupling system having two planetary members;

fig $5 \mathrm{c}$ the embodiment of $5 \mathrm{~b}$ at maximum stiffness, and .

fig $5 \mathrm{~d}$ an embodiment of $5 \mathrm{~b}$ adjusted at a stiffness between maximum stiffness and zero stiffness.

\section{Detailed description of embodiments}

In the figures 1 and 2 there is shown a coupling system 1 for transmitting torque around

10 a central axis 2 of the coupling system. The coupling system 1 is suitable for coupling links of a robotic system (not shown)

The coupling system 1 comprises a stator 3 . It will be clear that in use of the coupling system 1 , the stator will not necessarily be stationary but however dynamics of the coupling system 1 will be described with respect to said stator 3 .

The coupling system 1 comprises a hypocycloid transmission system 4 . The hypocycloid transmission system 4 is rotatably supported by the stator 3 . The entire hypocycloid transmission system 4 , also referred to with rotor, may rotate around the central axis 2 .

The coupling system 1 comprises an output member 5 for transmitting torque to a

20 robotic system part (not shown). The output member 5 is rotatably coupled with the stator 3 through the hypocycloid transmission system 4 .

The hypocycloid transmission system 4 comprises two planetary wheel members 6. The two planetary wheel members 6 mutually engage at the central axis in a driving manner. The engagement of the two wheel members 6 is shown schematically,

25 however any engagement suitable for driving is conceivable like tooth engagement, effect of friction, rolling link mechanism, etc.

The hypocycloid transmission system 4 comprise a carrier 11 . The carrier 11 supports the two planetary wheel member 6 . The hypocycloid transmission system 4 comprises an annular member 12 . The annular member 12 engages the two planetary

30 wheel member in a driving manner. Again, the engagement of the annular member 12 and the two wheel members 6 is shown schematically, however any engagement suitable for driving is conceivable. The wheel members 6 engage the annular member 12 at an inner circumference 13 of the annular member 12 . The two wheel members 6 
have a diameter $1 / 2 \mathrm{D}$ which is half the diameter $\mathrm{D}$ of the inner circumference 13 of the annular member 12 .

The wheel member 6 , here each of the two wheel members 6 , are provided with a drive cam 7 . The drive cam 7 is integrally formed with the planetary wheel member 6 . The drive cam 7 is provided at the outer circumference of the wheel member 6 . The drive cam 7 extends axially from the wheel member 6 and parallel with respect to the central axis 2 . The radial position of the drive cam 7 with respect to the central axis 2 is adjustable through the hypocycloid transmission system 4 .

As schematically shown in fig. 3 , the radial position of the drive cam 7 with respect to the bending member 8 is adjustable through rotating the carrier 11 with respect to the annular member 12 .

When the inner position of the drive cam 7 or cams 7 coincides with the central axis 2 , the output member 5 may rotate freely around the drive cam 7 . Effectively, the

15 output member is uncoupled from the rotor. In case of two drive cams 7 the drive cams 7 are configured to mate, as shown in fig. 1, in their inner position at the central axis such that the output member may rotate freely with respect to the hypocycloid transmission. Here, the drive cams 7 are configured to form a cylinder when they mate in their inner position at the central axis 2 .

In fig. $5 \mathrm{a}$, the drive cam 7 is pivotable with respect to the planetary wheel member 6 . The cam 7 is provided with opposite contact areas (not shown in detail) for driving the output member 8 both clockwise and counter clockwise.

25 As shown in fig. 4 and 5 , the output member 5 comprises a bending member 8 . In use, the drive cams 7 contact the bending member 8 in a driving manner. The drive cam 7 and the bending member 8 contact in a bearing manner. The bending member 8 is at one 9 end thereof coupled with the output member 5 at an outer circumference 10 of the output member 5 . The bending member 8 extends from the outer circumference 10

30 of the output member 5 towards the central axis 2 . As mentioned, the drive cam 7 contacts the bending member 8 for driving the output member 5 . The radial position of the drive cam 7 with respect to the bending member 8 is adjustable through the hypocycloid transmission system 4 . The radial position of the drive cam 7 is adjustable 
between an outer position, shown in fig. $5 \mathrm{c}$, proximate the circumference of the output 5 member and an inner position, shown in e.g. fig 1, proximate the central axis 2 . The stiffness of the coupling system 1 around its central axis 2, depends on the radial position of the drive cams 7 . In fig $5 \mathrm{c}$ the coupling system is adjusted at maximum

5 stiffness, and in fig $5 \mathrm{~d}$ the coupling system is adjusted at a stiffness between maximum stiffness and zero stiffness.

As shown in fig. $4 \mathrm{~b}, 5 \mathrm{a}$ the bending element 8 extends diametrical with respect to the central axis 2 and the hypocycloid transmission system 4 . Here, these two bending member enclose the drive cam 7 or cams.

As shown in fig. $4 \mathrm{e}$ and $5 \mathrm{~b}$, the two bending elements 8 constitute a slit 14 between them for receiving the drive cam 7 or cams 7 .Here, the slit 14 extends

15 diametrical with respect to the central axis 2 and the hypocycloid transmission system 4. In this case, as shown in fig. $4 \mathrm{e}$, the two bending elements 8 are integrally formed to constitute the slit 14 between them.

It will also be obvious after the above description and drawings are included to

20 illustrate some embodiments of the invention, and not to limit the scope of protection. Starting from this disclosure, many more embodiments will be evident to a skilled person which are within the scope of protection and the essence of this invention and which are obvious combinations of prior art techniques and the disclosure of this patent. 


\section{Claims}

1. Coupling system (1) for transmitting torque around a central axis (2) of the coupling system, wherein the coupling system comprises;

- a stator (3),

- a hypocycloid transmission system (4) rotatably supported by the stator,

- an output member (5) for transmitting torque, which output member is rotatably coupled with the stator through the hypocycloid transmission system,

wherein, the hypocycloid transmission system comprises a planetary wheel member (6) provided with a drive cam (7), the output member comprises a bending member (8) at one (9) end thereof coupled with the output member at an outer circumference (10) thereof which bending member extends from the outer circumference of the output member towards the central axis, the drive cam contacts the bending member for driving the output member, and the radial position of the drive cam with respect to the bending member is adjustable through the hypocycloid transmission system, between an outer position proximate the circumference of the output member and an inner position proximate the central axis, for adjusting a desired stiffness of the coupling system.

2. Coupling system (1) according to claim 1, wherein the hypocycloid transmission system comprise a carrier (11) supporting the planetary wheel member, and an annular member (12) which engages the planetary wheel member at an outer circumference thereof in a driving manner, and wherein the radial position of the drive cam with respect to the bending member is adjustable through rotating the carrier with respect to the annular member.

3. Coupling system (1) according to a preceding claim, wherein the inner position of the drive cam coincides with the central axis such that the output member may rotate freely around the drive cam. 
4. Coupling system (1) according to a preceding claim, wherein the hypocycloid transmission system is a planetary gear system.

5. Coupling system (1) according to a preceding claim, wherein the hypocycloid transmission system comprises two planetary wheel members which mutually engage at the central axis in a driving manner.

6. Coupling system (1) according to a preceding claim, wherein the drive cam is integrally formed with the planetary wheel member.

10

7. Coupling system (1) according to a preceding claim 1-5, wherein the drive cam is pivotable with respect to the planetary wheel member.

8. Coupling system (1) according to a preceding claim, wherein the bending element extends diametrical with respect to the central axis and the hypocycloid transmission system.

9. Coupling system (1) according to a preceding claim, comprising two bending elements

10. Coupling system (1) according to claim 9, wherein the two bending elements constitute a slit between them for receiving the cam or cams.

11. Coupling system (1) according to claim 10, wherein the slit extends diametrical with respect to the central axis and the hypocycloid transmission system.

12. Coupling system (1) according to a preceding claim 9-11, wherein two bending elements are integrally formed.

30 13. Coupling system (1) according to a preceding claim 5-12, wherein the two planetary wheel members are each provided with a drive cam. 
14. Coupling system (1) according to claim 13 , wherein the drive cams are configured to mate in their inner position at the central axis such that the output member may rotate freely with respect to the hypocycloid transmission.

5 15. Coupling system (1) according to claim 14, wherein the drive cams are configured to form a cylinder when they mate in their inner position at the central axis. 


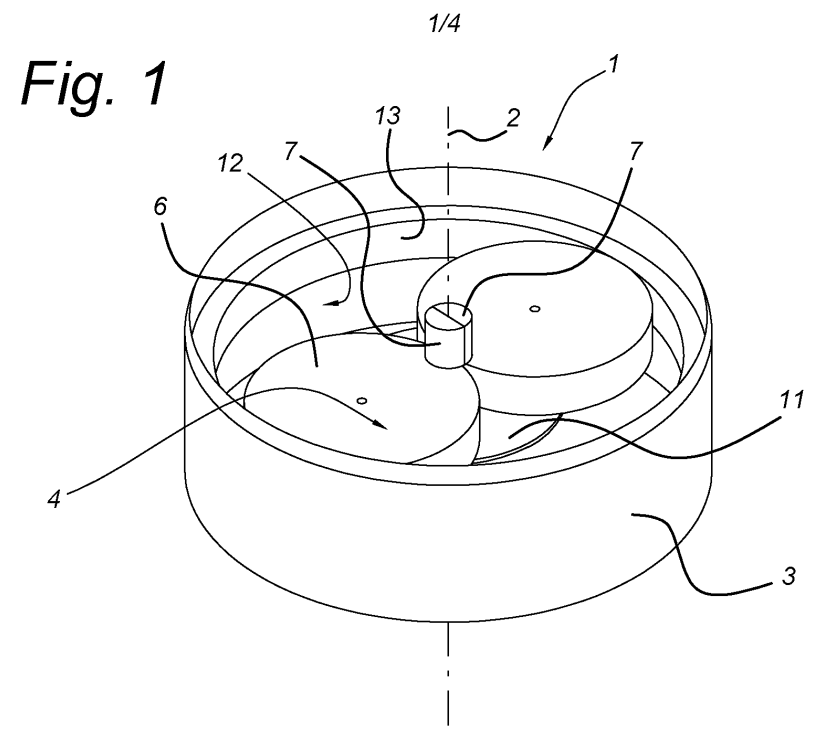

Fig. 2

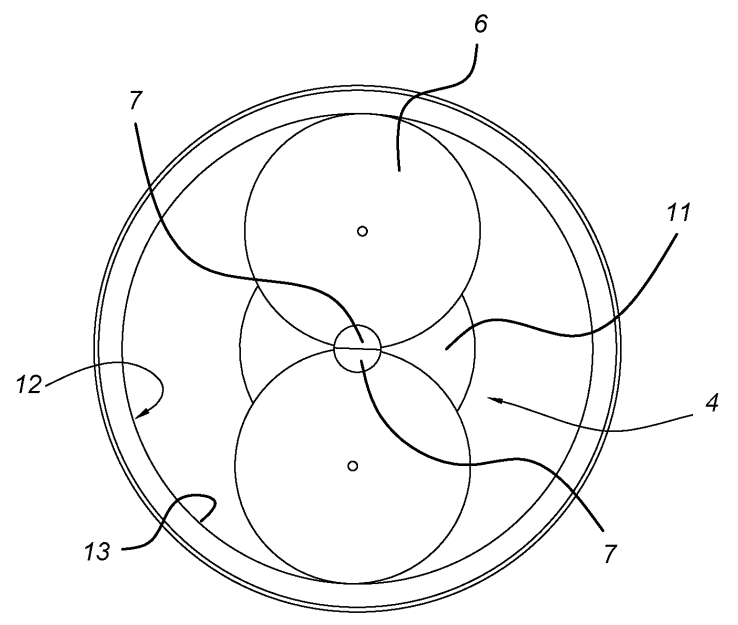


Fig. 3

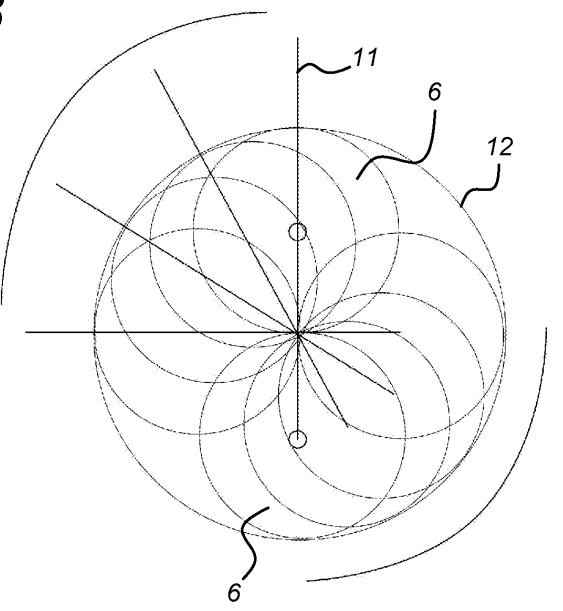

Fig. $4 a$

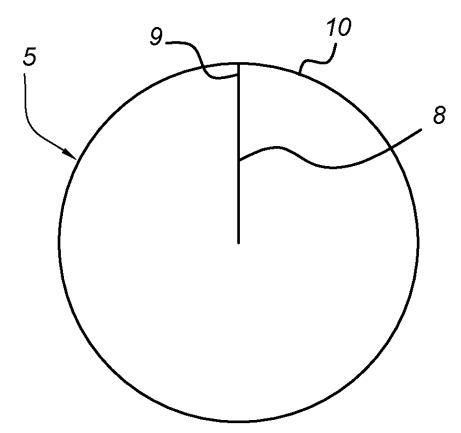

Fig. $4 b$

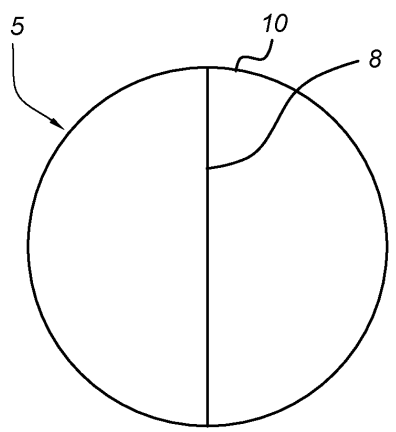


Fig. 4C

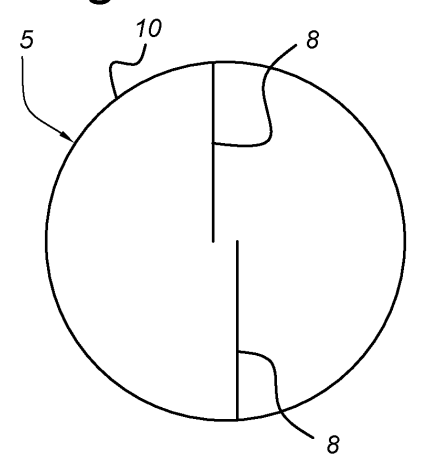

Fig. $4 e$

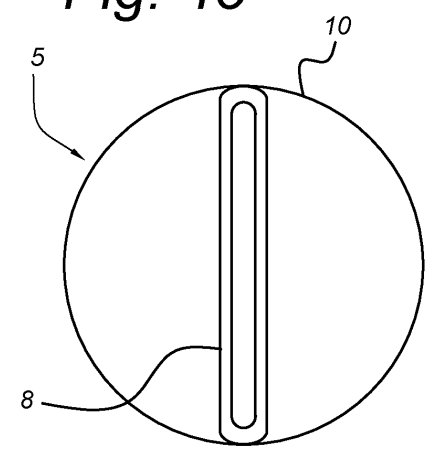

Fig. $4 d$

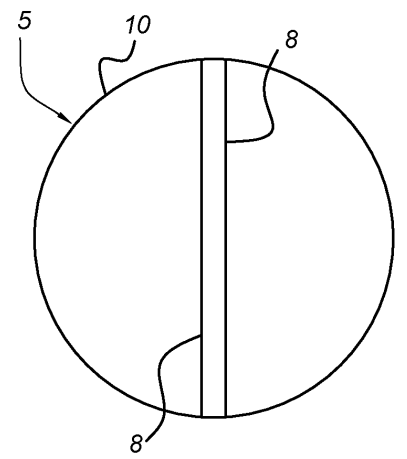

Fig. 5a

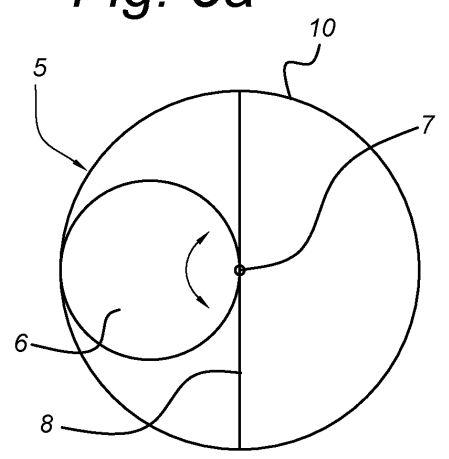


Fig. $5 b$

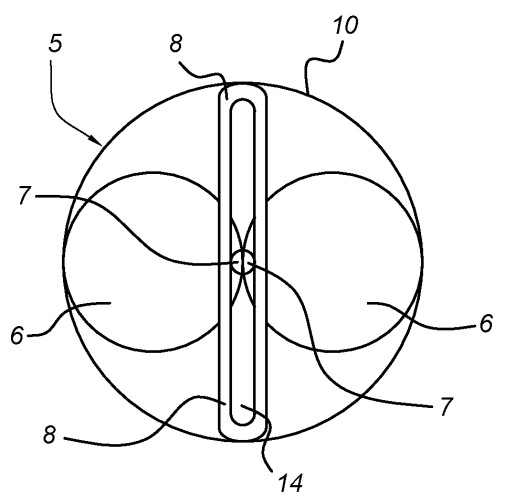

Fig. 5c

Fig. $5 d$
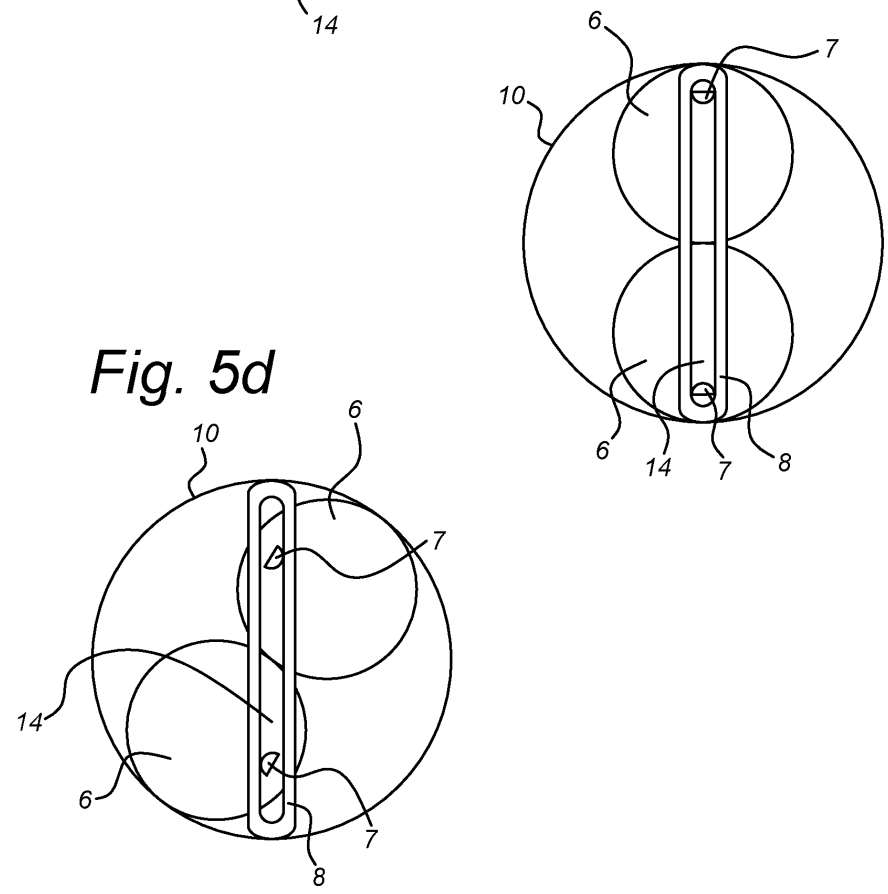
INTERNATIONAL SEARCH REPORT

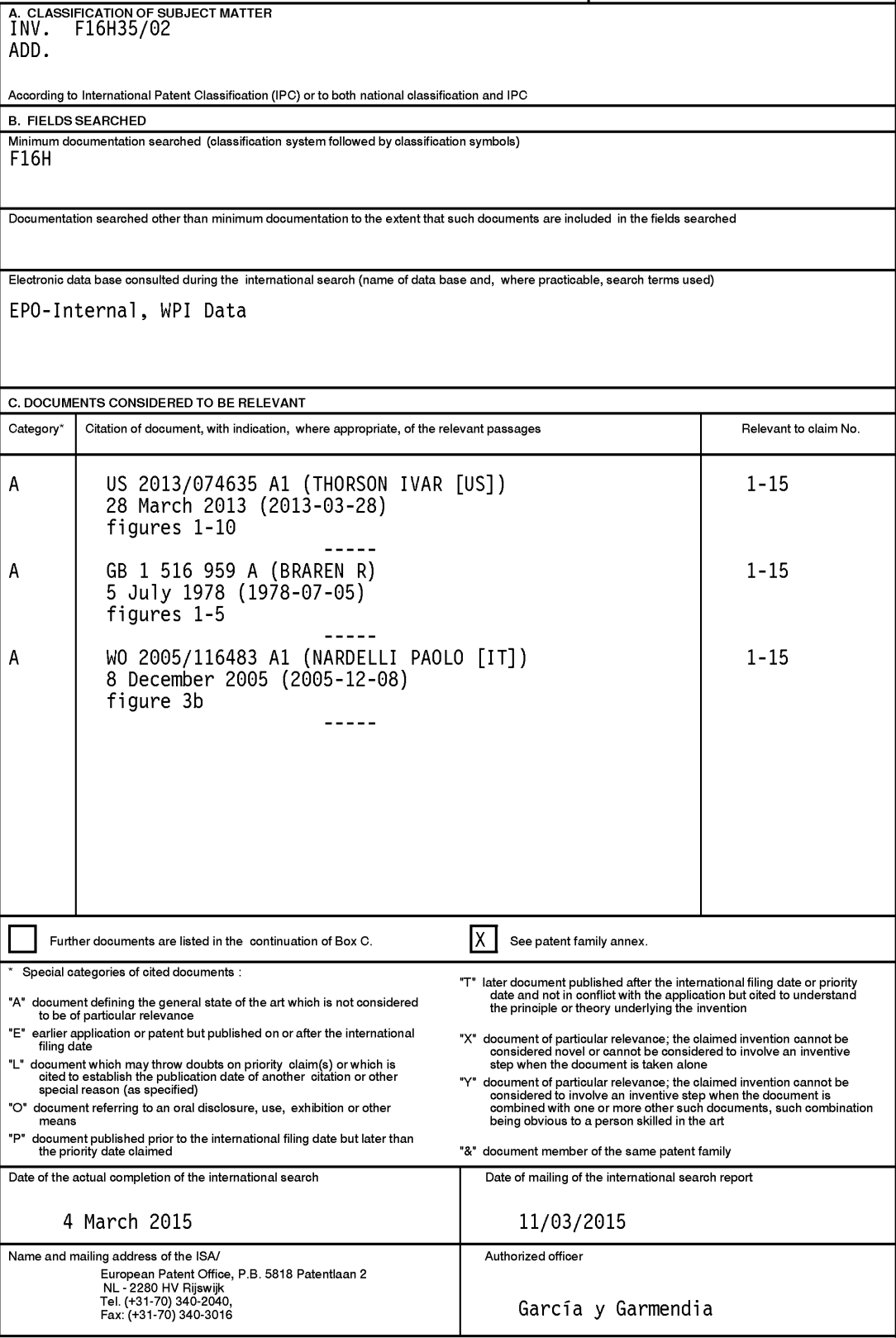




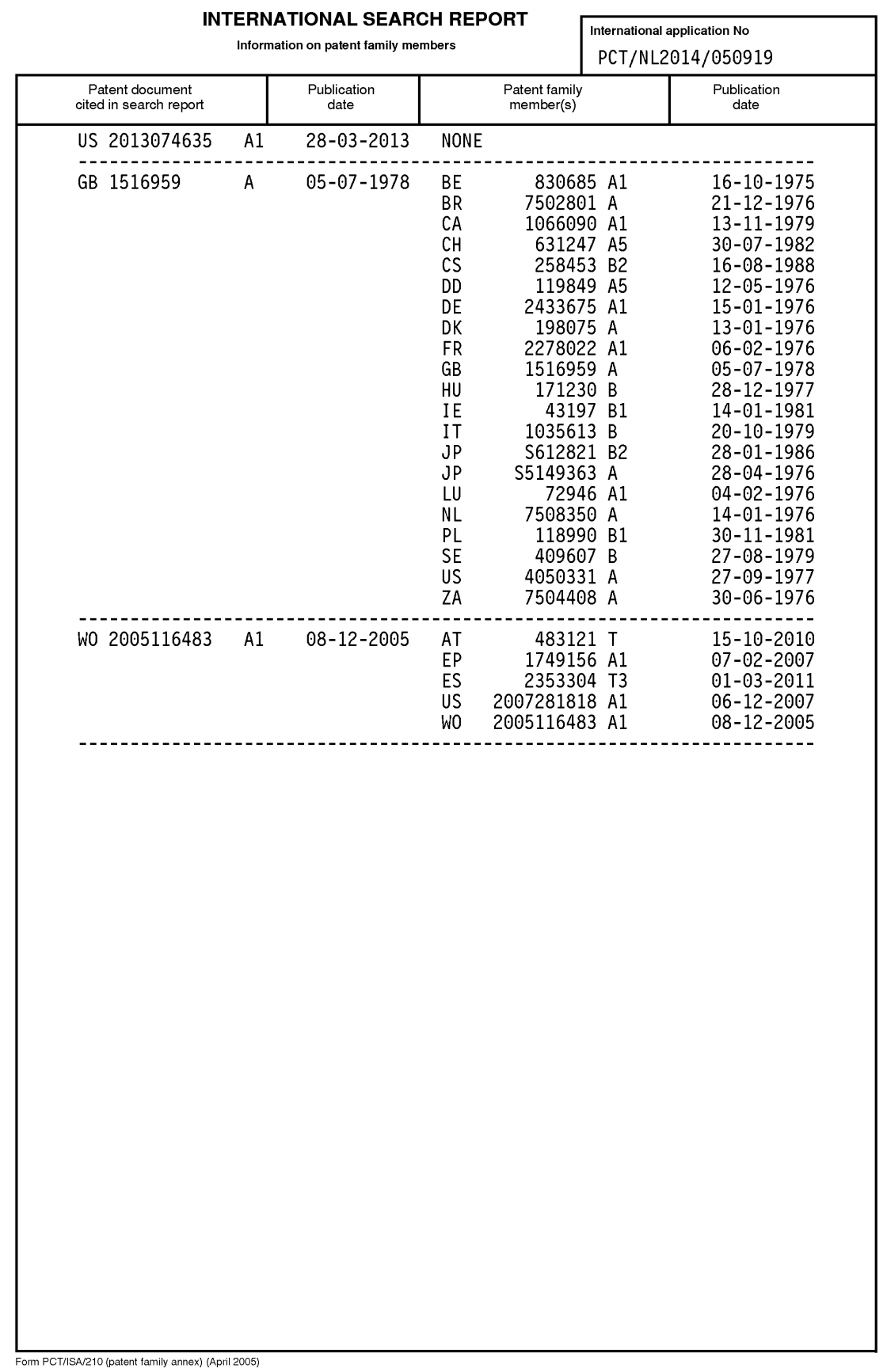




\section{Bibliography}

[1] L. Marconi et al., "The SHERPA project: Smart collaboration between human and ground-aerial robots for improving rescuing activities in alpine environments," in Proceedings of the IEEE International Symposium on Safety, Security, and Rescue Robotics (SSRR), pp. 1-4, 2012.

[2] M. de Klerk, H. Fernee, I. Woittiez, and M. Ras, "Mensen met lichamelijke of verstandelijke beperkingen." Online, 2011. Sociaal en Cultureel Planbureau Factsheet.

[3] Wikipedia, "Numeral prefix." Online, January 2016.

[4] S. Groothuis, S. Stramigioli, and R. Carloni, "Lending a helping hand," IEEE Robotics and Automation Magazine, vol. 20, no. 1, pp. 20-29, 2013. (c) 2013 IEEE.

[5] G. R. B. E. Römer, H. J. A. Stuyt, and A. Peters, "Cost-savings and economic benefits due to the assistive robotic manipulator (ARM)," in Proceedings of the IEEE International Conference on Rehabilitation Robotics, 2005.

[6] C. A. Stanger, C. Anglin, W. S. Harwin, and D. P. Romilly, "Devices for assisting manipulation: a summary of user task priorities," IEEE Transactions on Rehabilitation Engineering, vol. 2, no. 4, pp. 256-265, 1994.

[7] Z. Bien, M. J. Chung, P. H. Chang, D. S. Kwon, D. J. Kim, J. S. Han, J. H. Kim, D. H. Kim, H. S. Park, S. H. Kang, K. Lee, and S. C. Lim, "Integration of a rehabilitation robotic system (KARES II) with human-friendly man-machine interaction units," Autonomous Robots, vol. 16, pp. 165-191, 2004.

[8] S. D. Prior, "An electric wheelchair mounted robotic arm - a survey of potential users," Journal of Medical Engineering and Technology, vol. 14, no. 4, pp. 143-154, 1990.

[9] J. Hammel, K. Hall, D. Lees, L. Leifer, M. van der Loos, I. Perkash, and R. Crigler, "Clinical evaluation of a desktop robotic assistant," Journal of Rehabilitation Research, vol. 26, no. 3, pp. 1-16, 1989.

[10] S. Allin, E. Eckel, H. Markham, and B. R. Brewer, "Recent trends in the development and evaluation of assistive robotic manipulation devices," Physical Medicine and Rehabilitation Clinics of North America, vol. 21, no. 1, pp. 59 - 77, 2010. 
[11] J. Versace, "A review of the severity index," SAE Technical Paper 710881, 1971.

[12] M. Wassink and S. Stramigioli, "Towards a novel safety norm for domestic robotics," in Proceedings of the IEEE International Conference on Intelligent Robots and Systems, 2007.

[13] ISO, "ISO/DIS 13482-40.99: Robots and robotic devices - safety requirements for nonindustrial robots - non-medical personal care robot," 2012.

[14] Kinova, "JACO Arm user guide," 2010. http://www.robotshop.com.

[15] Assistive Innovations, "iARM intelligent arm robot manipulator." Brochure, 2010. http://assistive-innovations.com.

[16] M. Hillman, K. Hagan, S. Hagan, J. Jepson, and R. Orpwood, "The Weston wheelchair mounted assistive robot - the design story," Robotica, vol. 20, pp. 125-132, 2002.

[17] FOCAL Meditech B.V., "Personal Robot Bridgit." Online, 2012. http://www.focalmeditech.nl.

[18] W. K. Yoon, "Robotic Arm for Persons with Upper-limb DisAbilities (RAPUDA)," AIST Today, vol. 10, no. 2, p. 17, 2010.

[19] N. Lauzier and C. Gosselin, "Series clutch actuators for safe physical human-robot interaction," in Proceedings of the IEEE International Conference on Robotics and Automation, 2011.

[20] Barrett Technology Inc., "WAM Arm datasheet." Online, 2012. http://www.barrett.com.

[21] N. Hogan, "Impedance control: An approach to manipulation: Part i, part ii, part iii," ASME Journal of Dynamic Systems, Measurement and Control, 1985.

[22] Elumotion, "Elumotion RT2-Arm." Online, 2012. http://www.elumotion.com.

[23] G. Hirzinger, N. Sporer, A. Albu-Schäffer, M. Hahnle, R. Krenn, A. Pascucci, and M. Schedl, "DLR's torque-controlled light weight robot III-are we reaching the technological limits now?," in Proceedings of the IEEE International Conference on Robotics and Automation, 2002.

[24] M. S. Johannes, J. D. Bigelow, J. M. Burck, S. D. Harshbarger, M. V. Kozlowski, and T. van Doren, "An overview of the developmental process for the modular prosthetic limb," Johns Hopkins APL Technical Digest, vol. 30, no. 3, pp. 207-216, 2011.

[25] M. Grebenstein, A. Albu-Schäffer, T. Bahls, M. Chalon, O. Eiberger, W. Friedl, R. Gruber, S. Haddadin, U. Hagn, R. Haslinger, H. Höppner, S. Jörg, M. Nickl, A. Nothhelfer, F. Petit, J. Reill, N. Seitz, T. Wimböck, S. Wolf, T. Wusthoff, and G. Hirzinger, "The DLR hand arm system," in Proceedings of the IEEE International Conference on Robotics and Automation, 2011.

[26] M. A. Diftler, J. S. Mehling, M. E. Abdallah, N. A. Radford, L. B. Bridgwater, A. M. Sanders, R. S. Askew, D. M. Linn, J. D. Yamokoski, F. A. Permenter, B. K. Hargrave, R. Piatt, R. T. Savely, and R. O. Ambrose, "Robonaut 2 - The first humanoid robot in space," in Proceedings of the IEEE International Conference on Robotics and Automation, 2011. 
[27] R. van Ham, T. G. Sugar, B. Vanderborght, K. W. Hollander, and D. Lefeber, "Compliant actuator designs," IEEE Robotics and Automation Magazine, vol. 16, no. 3, pp. 81-94, 2009.

[28] T. Morita and S. Sugano, "Development and Evaluation of Seven-D.O.F. MIA ARM," in Proceedings of the IEEE International Conference on Robotics and Automation, 1997.

[29] P. Schrock, F. Farelo, R. Alqasemi, and R. Dubey, "Design, simulation and testing of a new modular wheelchair mounted robotic arm to perform activities of daily living," in Proceedings of the IEEE International Conference on Rehabilitation Robotics, 2009.

[30] IAT, "Project FRIEND III." Online, 2012. http://www.iat.uni-bremen.de.

[31] M. Fridenfalk, U. Lorentzon, and G. Bolmsjö, "Virtual prototyping and experience of modular robotics design based on user involvement," in Proceedings of the European Conference for the Advancement of Assistive Technology, 1999.

[32] Liberating Technologies, Inc., "LTI Boston Arm Systems." Online, 2012. http://www.liberatingtech.com.

[33] DEKA Research, “The DEKA Arm.” Online, 2012. http://www.dekaresearch.com.

[34] R. M. Mahoney, "The Raptor wheelchair robot system," in Proceedings of the IEEE International Conference on Rehabilitation Robotics, 2001.

[35] F. Petit and A. Albu-Schäffer, "State feedback damping control for a multi DOF variable stiffness robot arm," in Proceedings of the IEEE International Conference on Robotics and Automation, 2011.

[36] A. Jafari, N. G. Tsagarakis, and D. G. Caldwell, "Exploiting natural dynamics for energy minimization using an Actuator with Adjustable Stiffness (AwAS)," in Proceedings of the IEEE International Conference on Robotics and Automation, 2011.

[37] L. Visser, R. Carloni, and S. Stramigioli, "Energy efficient control of robots with variable stiffness actuators," in Proceedings of the IFAC International Symposium on Nonlinear Control Systems, 2010.

[38] L. Visser, R. Carloni, and S. Stramigioli, "Energy-efficient variable stiffness actuators," IEEE Transactions on Robotics, vol. 27, no. 5, pp. 865-875, 2011.

[39] S. Groothuis, G. Rusticelli, A. Zucchelli, S. Stramigioli, and R. Carloni, "The variable stiffness actuator vsaUT-II: Mechanical design, modeling and identification," IEEE/ASME Transactions on Mechatronics, vol. 19, no. 2, pp. 589-597, 2014. (c) 2014 IEEE.

[40] M. Uemura and S. Kawamura, "Resonance-based motion control method for multijoint robot through combining stiffness adaptation and iterative learning control," in Proceedings of the IEEE International Conference on Robotics and Automation, pp. 15431548, 2009.

[41] B. Vanderborght, N. Tsagarakis, C. Semini, R. van Ham, and D. Caldwell, "MACCEPA 2.0: Adjustable compliant actuator with stiffening characteristic for energy efficient hopping," in Proceedings of the IEEE International Conference on Robotics and Automation, pp. 544-549, 2009. 
[42] R. Schiavi, G. Grioli, S. Sen, and A. Bicchi, "VSA-II: A novel prototype of variable stiffness actuator for safe and performing robots interacting with humans," in Proceedings of the IEEE International Conference on Robotics and Automation, pp. 2171-2176, 2008.

[43] S. Wolf and G. Hirzinger, "A new variable stiffness design: Matching requirements of the next robot generation," in Proceedings of the IEEE International Conference on Robotics and Automation, pp. 1741-1746, 2008.

[44] T. Morita and S. Sugano, "Development of an anthropomorphic force-controlled manipulator WAM-10," in Proceedings of the IEEE International Conference on Advanced Robotics, pp. 701-706, 1997.

[45] J. Choi, S. Hong, W. Lee, and S. Kang, "A variable stiffness joint using leaf springs for robot manipulators," in Proceedings of the IEEE International Conference on Robotics and Automation, pp. 4363-4368, 2009.

[46] K. Hollander, T. Sugar, and D. Herring, "Adjustable robotics tendon using a 'Jack Spring'TM," in Proceedings of the International Conference on Rehabilitation Robotics, pp. 113-118, 2005.

[47] B.-S. Kim and J.-B. Song, "Hybrid dual actuator unit: A design of a variable stiffness actuator based on an adjustable moment arm mechanism," in Proceedings of the IEEE International Conference on Robotics and Automation, pp. 1655-1660, 2010.

[48] A. Jafari, N. Tsagarakis, B. Vanderborght, and D. Caldwell, "A novel actuator with adjustable stiffness (AwAS)," in Proceedings of the IEEE/RSJ International Conference on Intelligent Robots and Systems, pp. 4201-4206, 2010.

[49] A. Jafari, N. Tsagarakis, B. Vanderborght, and D. Caldwell, "AwAS-II: A new actuator with adjustable stiffness based on the novel principle of adaptable pivot point and variable lever ratio," in Proceedings of the IEEE International Conference on Robotics and Automation, pp. 4638-4643, 2011.

[50] N. G. Tsagarakis, I. Sardellitti, and D. G. Caldwell, "A new variable stiffness actuator (CompAct-VSA): Design and modelling," in Proceedings of the IEEE/RSJ International Conference on Intelligent Robots and Systems, pp. 378-383, 2011.

[51] M. Fumagalli, E. Barrett, S. Stramigioli, and R. Carloni, "The mVSA-UT: a miniaturized differential mechanism for a continuous rotational variable stiffness actuator," in Proceedings of the IEEE/EMBS International Conference on Biomedical Robotics and Biomechatronics, pp. 1943-1948, 2012.

[52] S. Rao, R. Carloni, and S. Stramigioli, "A novel energy-efficient rotational variable stiffness actuator," in Proceedings of the IEEE/EMBS International Conference of Engineering in Medicine and Biology Society, pp. 8175-8178, 2011.

[53] L. C. Visser, R. Carloni, and S. Stramigioli, "Variable stiffness actuators: a port-based analysis and a comparison of energy efficiency," in Proceedings of the IEEE International Conference on Robotics and Automation, pp. 3279-3284, 2010.

[54] R. Carloni, L. C. Visser, and S. Stramigioli, "Variable stiffness actuators: A port-based power-flow analysis," IEEE Transactions on Robotics, vol. 28, no. 1, pp. 1-11, 2012. 
[55] S. Groothuis, G. Rusticelli, A. Zucchelli, S. Stramigioli, and R. Carloni, "The vsaUT-II: A novel rotational variable stiffness actuator," in Proceedings of the IEEE International Conference on Robotics and Automation, 2012.

[56] S. Groothuis, "Design, modeling and control of a rotational variable stiffness actuator," Master's thesis, University of Twente, 2011. https://www.ce.utwente.nl/aigaion/publications/show/2003.

[57] I. Thorson and D. Caldwell, "A nonlinear series elastic actuator for highly dynamic motions," in Proceedings of the IEEE/RSJ International Conference on Intelligent Robots and Systems, pp. 390-394, 2011.

[58] D. W. Dudley, Gear Handbook. McGraw-Hill books, 1962.

[59] Maxon Motor, http://www.maxonmotor.com, "Maxon program," 2012.

[60] C. Pelchen, C. Schweiger, and M. Otter, "Modeling and simulating the efficiency of gearboxes and of planetary gearboxes," in Proceedings of the International Modelica Conference, pp. 257-266, 2002.

[61] Austria Microsystems, "AS5043 datasheet." Online, April 2009.

[62] S. Groothuis, R. Carloni, and S. Stramigioli, "A novel variable stiffness mechanism capable of an infinite stiffness range and unlimited decoupled output motion," Actuators, vol. 3, no. 2, pp. 107-123, 2014.

[63] S. Stramigioli and S. S. Groothuis, "WO2015102490 A1: Variable Stiffness Actuator (patent pending)," 2015.

[64] B. Vanderborght, A. Albu-Schaeffer, A. Bicchi, E. Burdet, D. Caldwell, R. Carloni, M. Catalano, O. Eiberger, W. Friedl, G. Ganesh, M. Garabini, M. Grebenstein, G. Grioli, S. Haddadin, H. Hoppner, A. Jafari, M. Laffranchi, D. Lefeber, F. Petit, S. Stramigioli, N. Tsagarakis, M. van Damme, R. van Ham, L. Visser, and S. Wolf, "Variable impedance actuators: a review," Robotics and Autonomous Systems, vol. 61, pp. 1601-1614, 2013.

[65] J. Choi, S. Hong, W. Lee, and S. Kang, "A robot joint with variable stiffness using leaf springs," IEEE Transactions on Robotics, vol. 27, no. 2, pp. 229-238, 2011.

[66] S. S. Groothuis, R. Carloni, and S. Stramigioli, "Single motor-variable stiffness actuator using bistable switching mechanisms for independent motion and stiffness control," in Proceedings of the IEEE International Conference on Advanced Intelligent Mechatronics, 2016. Submitted.

[67] E. J. Rouse, L. M. Mooney, E. C. Martinez-Villalpando, and H. M. Herr, "Clutchable series-elastic actuator: Design of a robotic knee prosthesis for minimum energy consumption," in Proceedings of the IEEE International Conference on Rehabilitation Robotics, pp. 1-6, 2013.

[68] N. Kashiri, M. Laffranchi, N. G. Tsagarakis, I. Sardellitti, and D. G. Caldwell, "Dynamic modeling and adaptable control of the compact ${ }^{\mathrm{TM}}$ arm," in Proceedings of the IEEE International Conference on Mechatronics, pp. 477-482, 2013.

[69] N. Kashiri, M. Laffranchi, N. G. Tsagarakis, A. Margan, and D. G. Caldwell, "Physical interaction detection and control of compliant manipulators equipped with friction clutches," in Proceedings of the IEEE International Conference on Robotics and Automation, pp. 1066-1071, 2014. 
[70] H. Tomori, Y. Midorikawa, and T. Nakamura, "Derivation of nonlinear dynamic model of novel pneumatic artificial muscle manipulator with a magnetorheological brake," in IEEE International Workshop on Advanced Motion Control, pp. 1-8, 2012.

[71] C. Berthelette, M. DiPinto, and J. Sareault, "Rotary "one-to-many" (otm) novel actuator," BSc. Thesis, Worcester Polytechnic Institute, 2013.

[72] T. Hunt, C. Berthelette, and M. Popovic, "Linear one-to-many (otm) system," in IEEE International Conference on Technologies for Practical Robot Applications (TePRA), pp. 16, April 2013.

[73] M. Cempini, M. Fumagalli, N. Vitiello, and S. Stramigioli, "A clutch mechanism for switching between position and stiffness control of a variable stiffness actuator," in Proceedings of the IEEE International Conference on Robotics and Automation, pp. 10171022, 2015.

[74] W. C. Orthwein, Clutches and Brakes - Design and Selection. Marcel Dekker, Inc., second ed., 2004.

[75] R. C. Hibbeler, Engineering Mechanics: Statics and Dynamics. Pearson Prentice Hall, 12th ed., 2010.

[76] N. H. Beachley and A. A. Frank, "Continuous variable transmission: Theory and practice," tech. rep., College of Engineering, University of Wisconsin, Madison, 1979.

[77] S. Groothuis, S. Stramigioli, and R. Carloni, "Compliant robotic systems on graphs," in Proceedings of the IEEE/RSJ International Conference on Intelligent Robots and Systems, 2014. (c) 2014 IEEE.

[78] D. Braun, M. Howard, and S. Vijayakumar, "Optimal variable stiffness control: formulation and application to explosive movement tasks," Autonomous Robots, vol. 33, no. 3, pp. 237-253, 2012.

[79] I. Sardellitti, G. Medrano-Cerda, N. Tsagarakis, A. Jafari, and D. Caldwell, "Gain scheduling control for a class of variable stiffness actuators based on lever mechanisms," IEEE Transactions on Robotics, vol. 29, no. 3, pp. 791-798, 2013.

[80] B. Berret, S. Ivaldi, F. Nori, and G. Sandini, "Stochastic optimal control with variable impedance manipulators in presence of uncertainties and delayed feedback," in Proceedings of the IEEE/RSJ International Conference on Intelligent Robots and Systems, pp. 4354-4359, 2011.

[81] W.-K. Chen, Graph Theory and Its Engineering Applications, vol. 5. World Scientific Publishing Co. Pte. Ltd., 1997.

[82] S. Stramigioli, Modeling and IPC control of interactive mechanical systems - A coordinatefree approach. Springer London, 2001.

[83] G. Baciu and H. K. Kesavan, "From particle-mass to multibody systems: Graphtheoretic modeling," IEEE Transactions on Systems, Man, and Cybernetics - Part A: Systems and Humans, vol. 27, no. 2, pp. 244-250, 1997.

[84] O. Shai and K. Preiss, "Graph theory representations of engineering systems and their embedded knowledge," Artificial Intelligence in Engineering, vol. 13, pp. 273-285, 1999. 
[85] A. van der Schaft and B. Maschke, "Port-hamiltonian systems on graphs," SIAM Journal on Control and Optimization, vol. 51, no. 2, pp. 906-937, 2013.

[86] A. Rahmani, M. Ji, M. Mesbahi, and M. Egerstedt, "Controllability of multi-agent systems from a graph-theoretic perspective," SIAM Journal on Control and Optimization, vol. 48, no. 1, pp. 162-186, 2009.

[87] D. Goldin, S. Attia, and J. Raisch, "Consensus for double integrator dynamics in heterogeneous networks," in Proceedings of the IEEE Conference on Decision and Control, pp. 4504-4510, 2010.

[88] W. Li and E. Todorov, "Iterative linear quadratic regulator design for nonlinear biological movement systems," in Proceedings of the International Conference on Informatics in Control, Automation and Robotics, pp. 222-229, 2004.

[89] D. J. Braun, F. Petit, F. Huber, S. Haddadin, P. van der Smagt, A. Albu-Schäffer, and S. Vijayakumar, "Robots driven by compliant actuators: Optimal control under actuation constraints," IEEE Transactions on Robotics, vol. 29, no. 5, pp. 1085-1101, 2013.

[90] S. S. Groothuis, S. Stramigioli, and R. Carloni, "Modeling robotic manipulators powered by variable stiffness actuators: a graph-theoretic and port-hamiltonian formalism," IEEE Transactions on Robotics, 2016. Submitted.

[91] S. Groothuis, S. Stramigioli, and R. Carloni, "Compliant manipulators on graphs," in Proceedings of the IEEE/RSJ International Conference on Intelligent Robots and Systems, 2015. (c) 2015 IEEE.

[92] G. Grioli, S. Wolf, M. Garabini, M. Catalano, E. Burdet, D. Caldwell, R. Carloni, W. Friedl, M. Grebenstein, M. Laffranchi, D. Lefeber, S. Stramigioli, N. Tsagarakis, M. V. Damme, B. Vanderborght, A. Albu-Schäffer, and A. Bicchi, "Variable stiffness actuators: The userś point of view," International Journal of Robotics Research, vol. 34, no. 6, pp. 727-743, 2015.

[93] A. De Luca and W. Book, "Robots with flexible elements," in Springer Handbook of Robotics (B. Siciliano and O. Khatib, eds.), pp. 287-319, Springer Berlin Heidelberg, 2008.

[94] M. W. Spong, "Modeling and control of elastic joint robots," Journal of Dynamic Systems, Measurement, and Control, vol. 109, no. 4, 1986.

[95] M. W. Spong, S. Hutchinson, and M. Vidyasagar, Robot Modeling and Control. John Wiley \& Sons, Inc., 2005.

[96] A. Voronov, "The roles of monoarticular and biarticular muscles of the lower limbs in terrestrial locomotion," Human Physiology, vol. 30, no. 4, pp. 476-484, 2004.

[97] A. M. Carroll and A. A. Biewener, "Mono- versus biarticular muscle function in relation to speed and gait changes: in vivo analysis of the goat triceps brachii," The Journal of Experimental Biology, vol. 212, no. 20, pp. 3349-3360, 2009.

[98] S. Tonetti and P. Masarati, "Graph-based modeling of nonhomogeneous onedimensional multibody systems with arbitrary topology," Journal of Computational Nonlinear Dynamics, vol. 6, no. 3, 2011. 
[99] S. Y. T. Lang and H. Kesavan, "Graph theoretic modeling and analysis of multibody planar mechanical systems," IEEE Transactions on Systems, Man and Cybernetics, Part A: Systems and Humans, vol. 31, no. 2, pp. 97-111, 2001.

[100] S. Lang and H. K. Kesevan, "Dynamics of planar mechanical systems: a graph theoretic approach," in Proceedings of the IEEE International Conference on Systems, Man, and Cybernetics, vol. 4, pp. 3077-3082, 1996.

[101] G. Baciu, J. Chou, and H. Kesavan, "Constrained multibody systems: graph-theoretic newton-euler formulation," IEEE Transactions on Systems, Man and Cybernetics, vol. 20, no. 5, pp. 1025-1048, 1990.

[102] M. Hai-Bo, L. Zhi-Guo, L. Yuan, W. Shi-Cheng, and Y. Yan-Li, "Coordination control of networked euler-lagrange systems with possible switching topology," Acta Automatica Sinica, vol. 39, no. 7, pp. 1003-1010, 2013.

[103] W. Ren, "Distributed leaderless consensus algorithms for networked euler-lagrange systems," International Journal of Control, vol. 82, no. 11, pp. 2137-2149, 2009.

[104] A. R. Hanzaki, S. K. Saha, and P. Rao, "An improved dynamic modeling of a multibody system with spherical joints," Multibody System Dynamics, vol. 21, no. 4, pp. 325-345, 2009.

[105] J. McPhee, "On the use of linear graph theory in multibody system dynamics," Nonlinear Dynamics, vol. 9, no. 1-2, pp. 73-90, 1996.

[106] J. Cáceres, M. Puertas, C. Hernando, M. Mora, I. Pelayo, and C. Seara, "Searching for geodetic boundary vertex sets," Electronic Notes in Discrete Mathematics, vol. 19, pp. 25-31, 2005.

[107] V. Duindam, A. Macchelli, S. Stramigioli, and H. Bruyninckx, eds., Modeling and Control of Complex Physical Systems: The Port-Hamiltonian Approach. Springer Science \& Business Media, 2009.

[108] J. Loncaric, Geometrical Analysis of Compliant Mechanisms in Robotics (Euclidean Group, Elastic Systems, Generalized Springs). PhD thesis, Harvard University, Cambridge, MA, USA, 1985.

[109] A. Macchelli, C. Melchiorri, and S. Stramigioli, "Port-based modeling and simulation of mechanical systems with rigid and flexible links," IEEE Transactions on Robotics, vol. 25, no. 5, pp. 1016-1029, 2009.

[110] S. S. Groothuis, S. Stramigioli, and R. Carloni, "Stiffness analysis and control of compliant robotic manipulators," IEEE Transactions on Robotics, 2016. Submitted.

[111] E. N. Marieb and K. Hoehn, Human Anatomy and Physiology. Benjamin Cummings, 7th ed., 2006.

[112] N. Hogan, "The mechanics of multi-joint posture and movement control," Biological Cybernetics, 1985.

[113] J. McIntyre, F. Mussa-Ivaldi, and E. Bizzi, "The control of stable postures in the multijoint arm," Experimental Brain Research, vol. 110, pp. 248-264, 1996. 
[114] H. Gomi and R. Osu, "Task-dependent viscoelasticity of human multijoint arm and its spatial characteristics for interaction with environments," The Journal of Neuroscience, vol. 18, pp. 8965-8978, November 1998.

[115] D. W. Franklin, G. Liaw, T. E. Milner, R. Osu, E. Burdet, and M. Kawato, "Endpoint stiffness of the arm is directionally tuned to instability in the environment," The Journal of Neuroscience, vol. 27, pp. 7705-7716, July 2007.

[116] S. Oh and Y. Hori, "Novel endeffector stiffness control by biarticular muscle in robot manipulator," in Proceedings of the 35th Annual Conference of the IEEE Industrial Electronics Society, pp. 3144-3149, 2009.

[117] H. Höppner, W. Wiedmeyer, and P. van der Smagt, "A new biarticular joint mechanism to extend stiffness ranges," in Proceedings of the IEEE International Conference on Robotics and Automation, pp. 3403-3410, 2014.

[118] C. Ott, A. Albu-Schäffer, A. Kugi, and G. Hirzinger, "Decoupling based cartesian impedance control of flexible joint robots," in Proceedings of the IEEE International Conference on Robotics and Automation, pp. 3101-3107, 2003.

[119] A. Albu-Schäffer, M. Fischer, G. Schreiber, F. Schoeppe, and G. Hirzinger, "Soft robotics: what cartesian stiffness can obtain with passively compliant, uncoupled joints?," in Proceedings of the IEEE/RSJ International Conference on Intelligent Robots and Systems, pp. 3295-3301, 2004.

[120] H. Kashiwagi, F. Okumura, S. Komada, and J. Hirai, "Stiffness ellipse control of tendon mechanisms with nonlinear springs," in Proceedings of the IEEE International Conference on Robotics and Biomimetics, pp. 1261-1266, 2011.

[121] A. Ajoudani, M. Gabiccini, N. Tsagarakis, A. Albu-Schäffer, and A. Bicchi, "Teleimpedance: Exploring the role of common-mode and configuration-dependant stiffness," in Proceedings of the 12th IEEE-RAS International Conference on Humanoid Robots, pp. 363-369, 2012.

[122] A. Ajoudani, N. Tsagarakis, and A. Bicchi, "On the role of robot configuration in cartesian stiffness control," in Proceedings of the IEEE International Conference on Robotics and Automation, pp. 1010-1016, 2015.

[123] K. L. Doty, C. Melchiorri, and C. Bonivento, "A theory of generalized inverses applied to robotics," The International Journal of Robotics Research, vol. 12, pp. 1-19, February 1993.

[124] K. L. Doty, C. Melchiorri, E. M. Schwartz, and C. Bonivento, "Robot manipulability," IEEE Transactions on Robotics and Automation, vol. 11, pp. 462-468, June 1995.

[125] M. Zefran and R. V. Kumar, "A geometric approach to the study of the cartesian stiffness matrix," Journal of Mechanical Design, vol. 124, pp. 30-38, March 2002.

[126] J. Kövecses and J. Angeles, "The stiffness matrix in elastically articulated rigid-body systems," Multibody System Dynamics, vol. 18, no. 2, pp. 169-184, 2007.

[127] J. Watrous, “Theory of Quantum Information: 2.3 Norms of operators." Lecture notes, 2011. 
[128] A. A. Maciejewski and C. A. Klein, "The singular value decomposition: Computation and applications to robotics," The International Journal of Robotics Research, vol. 8, no. 6, pp. 63-79, 1989.

[129] F. Petit and A. Albu-Schäffer, "Cartesian impedance control for a variable stiffness robot arm," in Proceedings of the IEEE/RSJ International Conference on Intelligent Robots and Systems, pp. 4180-4186, 2011.

[130] J. Selig, Geometrical Methods in Robotics. Springer-Verlag New York, 1996.

[131] R. Murray, Z. Li, and S. Sastry, A Mathematical Introduction to Robotic Manipulation. CRC Press, 1994.

[132] T. Rowland and E. W. Weisstein, "Commutator." MathWorld - A Wolfram Web Resource, 2015.

[133] J. E. Marsden and T. Ratiu, Introduction to Mechanics and Symmetry. Springer-Verlag New York, 1999.

[134] A. van der Schaft, "Port-hamiltonian systems: an introductory survey," in Proceedings of the International Congress of Mathematicians, 2006.

[135] M. Visser, S. Stramigioli, and C. Heemskerk, "Cayley-hamilton for roboticists," in Proceedings of the IEEE/RSJ International Conference on Intelligent Robots and Systems, 2006.

[136] H. M. Paynter, Analysis and design of engineering systems. The M.I.T. Press, Boston, 1961.

[137] P. C. Breedveld, "Bond graphs." Online, July 2003. Encyclopedia of Life Support Systems, Geoplex summer school.

[138] P. C. Breedveld, "Port-based modelling of multidomain physical systems in terms of bond graphs," in Simulation Techniques for Applied Dynamics (M. Arnold and W. Schiehlen, eds.), pp. 141-190, Springer Vienna, 2009.

[139] G. Golo, Interconnection Structures in Port-Based Modelling: Tools for Analysis and Simulation. PhD thesis, University of Twente, 2002.

[140] J. A. Primbs, Nonlinear optimal control: a receding horizon approach. PhD thesis, California Institute of Technology, 1999.

[141] R. M. Murray, “Optimization-based control.” Online, February 2010.

[142] R. E. Bellman, Dynamic Programming. Princeton University Press, 1957.

[143] H. Sussmann and J. Willems, "300 years of optimal control: from the brachystochrone to the maximum principle," IEEE Control Systems, vol. 17, no. 3, 1997.

[144] D. H. Jacobson and D. Q. Mayne, Differential Dynamic Programming. New York: American Elsevier Pub. Co., 1970.

[145] S. Yakowitz, Algorithms and Computation Techniques in Differential Dynamic Programming, vol. 31 of Control and Dynamical Systems: Advances in Theory and Applications, pp. 75-91. New York: Academic Press, 1989. 
[146] W. H. Kwon, A. Bruckstein, and T. Kailath, "Stabilizing state-feedback design via the moving horizon method," in Proceedings of the IEEE Conference on Decision and Control, vol. 21, pp. 234-239, 1982.

[147] G. Lantoine and R. P. Russell, "A hybrid differential dynamic programming algorithm for constrained optimal control problems, part I \& II," Journal of Optimization Theory and Applications, vol. 154, no. 2, pp. 283-442, 2012. 


\section{List of Publications}

This thesis is based on the following articles that have been published or have been submitted for possible publication in peer-reviewed journals or conference proceedings.

\section{Journal publications}

S. Groothuis, S. Stramigioli, and R. Carloni, "Lending a helping hand," IEEE Robotics and Automation Magazine, vol. 20, no. 1, pp. 20-29, 2013. (c) 2013 IEEE

S. Groothuis, G. Rusticelli, A. Zucchelli, S. Stramigioli, and R. Carloni, "The variable stiffness actuator vsaUT-II: Mechanical design, modeling and identification," IEEE/ASME Transactions on Mechatronics, vol. 19, no. 2, pp. 589-597, 2014. (c) 2014 IEEE

S. Groothuis, R. Carloni, and S. Stramigioli, "A novel variable stiffness mechanism capable of an infinite stiffness range and unlimited decoupled output motion," Actuators, vol. 3, no. 2, pp. 107-123, 2014

S. S. Groothuis, S. Stramigioli, and R. Carloni, "Modeling robotic manipulators powered by variable stiffness actuators: a graph-theoretic and port-hamiltonian formalism," IEEE Transactions on Robotics, 2016. Submitted

S. S. Groothuis, S. Stramigioli, and R. Carloni, "Stiffness analysis and control of compliant robotic manipulators," IEEE Transactions on Robotics, 2016. Submitted

\section{Patent publications}

S. Stramigioli and S. S. Groothuis, "WO2015102490 A1: Variable Stiffness Actuator (patent pending)," 2015

\section{Conference proceedings publications}

S. Groothuis, S. Stramigioli, and R. Carloni, "Compliant robotic systems on graphs," in Proceedings of the IEEE/RSJ International Conference on Intelligent Robots and Systems, 2014. (c) 2014 IEEE 
S. Groothuis, S. Stramigioli, and R. Carloni, "Compliant manipulators on graphs," in Proceedings of the IEEE/RSJ International Conference on Intelligent Robots and Systems, 2015. (c) $2015 \mathrm{IEEE}$

S. S. Groothuis, R. Carloni, and S. Stramigioli, "Single motor-variable stiffness actuator using bistable switching mechanisms for independent motion and stiffness control," in Proceedings of the IEEE International Conference on Advanced Intelligent Mechatronics, 2016. Submitted 


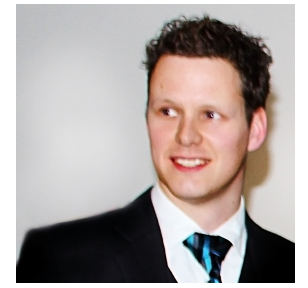

a bachelor (BSc.) in Electrical Engineering (2008), and a master (MSc.) in Mechatronics (2011), focusing on the modeling, design, and control of a variable stiffness actuator at the Robotics and Mechatronics (RaM) group. I spent my master's intership at The Flinders University of South Australia in Adelaide, Australia, in 2009, working on a vibration cancellation system using synthetic shunt impedances connected to electromagnetic transducers. After graduating my MSc., I continued at the RaM group to pursue a $\mathrm{PhD}$ degree on the modeling, design, and control of compliant robotic manipulators, as presented in this thesis. My research interests include novel mechanism design, energy-based modeling and simulation, manipulation, and human-robot interaction. In my spare time I like to play and listen to music (playing piano, at bit of bass, some percussion, and a tiny bit of singing), do sports (mountainbiking and running), watch movies, and drink beers with friends. 
Heel veel mensen hebben bijgedragen aan de succesvolle afronding van dit proefschrift, middels directe inhoudelijk inmenging of door simpelweg gezellig te zijn en juist niet te vragen naar mijn onderzoek. Geheel volgens traditie wil ik die mensen in dit dankwoord oprecht bedanken voor alle hulp en steun.

Allereerst bedank ik mijn begeleider en, vandaag, mijn promotor Stefano. Met recht kan ik zeggen dat dit project niet mogelijk was zonder jou, aangezien jij ervoor hebt gezorgd dat er budget werd gecreëerd waarmee ik aangesteld kon worden op dit project, dat ik uiteindelijk kon afsluiten met dit proefschrift. In de tweede helft van het project hadden we frequente, maar, om agendatechnische redenen, niet regelmatige, inhoudelijke discussies. Het inhoudelijke deel van de discussies kwam trouwens pas vaak nadat we tien minuten bijgepraat hadden over muziek: je studio-in-aanbouw-project (waar we als twee ingenieurs ook even konden nadenken over de deurconstructie), mijn nieuwe bassgitaar, techniekoefeningen voor de bass, algemene muziektheorie en jouw nieuwste "muziekgadgets" (looperpedaal, bassversterker, etc.). Deze voorliefde voor muziek bracht ons al eerder samen in jouw woonplaats Borne, om, samen met Seth, Pantelis en Nico, te gaan jammen in een studio, omdat Seth toevallig (of met voorbedachten rade), uitgenodigd was in Enschede om te opponeren tijdens een verdediging. Dit heeft tot diep in de nacht geduurd. Later, na IROS 2014 in Chicago, gingen we samen met Nanda en Alessandro naar het huis van Seth, om daar in zijn studio te jammen. Hoewel er wel sprake was van een generatiekloof in de muziekvoorkeur, heb ik me erg vermaakt. Wat ik bijzonder vind is dat we ook contact hadden over niet-werkgerelateerde situaties. Ik kon altijd bij jou aankloppen voor persoonlijke zaken en adviserende woorden. Ook nodigde je me uit bij jou thuis voor een barbecue op een willekeurige zaterdagmiddag. Het toeval wilde wel dat er nog een aanbouw omgetoverd moest worden tot de zojuist al genoemde studio, waarbij het noodzakelijk was om ongeveer $12 \mathrm{~m}^{3}$ grond te vervangen voor net zoveel bouwzand, waarbij ik dan mooi even zou kunnen helpen. ;) Zonder automatisering of robotica, gewoon handmatig. Alleszins heb je een belangrijk aandeel gehad in mijn ontwikkeling en hoop ik dat we contact kunnen houden in de toekomst.

I would like to thank my daily supervisor, and, today, my co-promotor, Raffaella. I have known you since I was shopping for MSc. assignments and picked yours which was within the VIACTORS project developing a variable stiffness actuator. That assignment has laid the basis for the work presented in this thesis. Since the beginning of my MSc. assignment up until the end of my PhD contract, we have had weekly meetings, in which explaining my issues and problems with various topics to you as a listener and discussion partner was often enough for 
me to get new insight. I guess there is some truth in the quote you mentioned a while ago: "If you have described the problem precisely, you have the solution.". You have put much effort into reading my papers and commenting on them and I can say that if it wasn't for you I would not have the publications that I have now. Also your guidance to me as an inexperienced $\mathrm{PhD}$ student during my daily work and at conferences was very helpful and I would also like to stay in touch with you.

Vandaag heb ik twee paranimfen die me bijstaan tijdens de verdediging, maar tijdens het project ben ik ook bijgestaan door twee andere paranimf-achtigen, namelijk de secretaresses en de technici van de vakgroep. Carla, Jolanda en Sandra, bedankt voor jullie hulp bij het regelen van de meest uiteenlopende zaken en het uit handen nemen van bepaalde onderwijstechnische zaken en administratieve rompslomp. Gerben, bedankt voor het meedenken bij de ontwikkeling van de prototypes, voor het tot in den treure en op korte termijn 3D-printen en lasersnijden van prototype-onderdelen en voor het even snel bedenken van ad hocoplossingen bij technische problemen. Marcel wil ik voornamelijk bedanken voor de ondersteuning bij allerlei praktische problemen in mijn meetopstellingen, schakelingen en systemen. Ook wist je vaak wel raad met mijn Linux-leerproces, waarin ik soms iets te naïef te werk ging: "\$ sudo chmod -R 0777 /etc", "Marcel, hij doet het niet meer..!". Henny, ook jij bedankt voor het helpen met allerlei problemen en uitdagingen die ik tijdens het ontwikkelen, realiseren en testen van prototypes tegenkwam.

Mijn collega's bij RAM, en mijn kamergenoten van de vierde en, later, de derde verdieping in het bijzonder, wil ik voornamelijk bedanken voor de leuke tijd die ik heb gehad bij de vakgroep. Iedereen was altijd wel in voor een geintje en met name met Roy, Dian, Guus en Gert hebben we menig "prank" uitgehaald. Niet alleen het volstoppen van Roy's bureauladen met confetti en papiersnippers (Hao: "We have to put a fire!"), of het aanleggen van een kruidentuintje op het iets te perfect ingerichte bureau van Guus, maar ook het instellen van een Glazenhuiscommissie voor het organiseren van een Serious Requestactie, waarbij Jos geheel onvrijwillig in zijn kantoor werd opgesloten met een variabele eindtijd die werd opgehoogd naarmate de vakgroep meer geld in de spaarpot deed: "Put in a dime, add half a minute to his time", waarmee we toch mooi ongeveer $€ 180$,- hebben opgehaald! Ook kon ik met collega's regelmatig discussies voeren over inhoudelijk problemen gerelateerd aan het project, of over grotere wereldkwesties die we tijdens de lunch even oplosten.

To my students, Giulia, Mariana, Julian, and Daniël: thanks that I could supervise you. I hope I have done well in guiding you and hope you look back on a pleasant stay at the RAM group. I think I have polished my supervising skills and methods along the way thanks to you.

Mijn vrienden wil ik bedanken voor het klaarstaan op de momenten dat het nodig of gewenst was, en uiteraard voor de altijd gezellige en frequente (speciaal)biertjes, bioscoopjes, barbecuetjes en feestjes, waarvan ik hoop dat er nog vele zullen volgen, ook al woon ik inmiddels niet meer op fietsafstand. Alle muzikanten van de bands en formaties waarin ik de afgelopen jaren heb gemusiceerd, en ook nu weer de gelegenheidsband voor het feest na een harmonieconcert: bedankt voor het plezier dat ik heb gehad, en nog steeds heb, tijdens repetities en optredens!

Pap en mam, ik had hier niet gestaan als jullie me niet altijd hadden gestimuleerd om te gaan studeren en me hadden gesteund toen ik er daadwerkelijk mee bezig was. Bedankt daarvoor! Dit hebben jullie vervolgens wel moeten bekopen met relatief beperkte informatie over hoe het nou eigenlijk ging met school (BSc. en MSc.) en de studie (PhD). Vaak volstond mijn oppervlakkige, maar toch in essentie wel juiste, "Oh, dat gaat wel prima hoor"-opmerking, soms aangevuld met enig detail. Gelukkig kweekte ik begrip hiervoor, door "Grad student etiquette" van PhD Comics te laten zien, maar ik denk dat jullie dat ook zelf al wel begrepen. Ook mijn schoonouders, -broertjes en -zusjes bedank ik voor hun steun en gezelligheid. 
Ik zei het al: ik word vandaag bijgestaan door twee paranimfen, Vincent en Olti. Vincent, jou bedank ik simpelweg omdat jij mijn broer bent die er altijd voor mij is. Ik kan altijd bij jou aankloppen voor allerlei zaken en je bent altijd bereid om te helpen, wat ik enorm waardeer. Wat me altijd erg dierbaar is wanneer we samen zijn, is het vaak ouwehoeren, en soms zelfs het zinnig discussiëren, over van alles. Niet zelden zijn pa en ma het lijdend voorwerp, waarin we elkaar feilloos aanvoelen en lekker ad rem meedoen met de onzinnigheid van dat moment. Ik vind het ook leuk om te merken dat we elkaar altijd heel goed begrijpen als het gaat om onze gezamenlijke passie: muziek. We hebben een tijd lang samen gemusiceerd in een vaste bandformatie en de laatste tijd heeft het een meer incidenteel karakter gekregen en gebeurt het in de vorm van gelegenheidsbands, die mede dankzij jou steeds een groot succes zijn. Ook vind ik het uiteraard fijn dat je vandaag naast me wilt staan als paranimf om me te steunen tijdens de verdediging. Je hebt veel van mijn voorbereidingstaken voor vandaag uit handen kunnen nemen, wat me een hoop geregel heeft bespaard. Ik hoop dat we alle dingen die we als broers doen, blijven volhouden en nog veel langer kunnen blijven doen. Olti, ik leerde je kennen tijdens het eerste jaar van de studie, en eigenlijk pas goed toen we een project samen deden (Mid-P: het ontwerpen van een stemapparaat). We konden goed door één deur en we hadden dezelfde humor en opvattingen, dus niet geheel toevallig deden we daarna meerdere projecten samen. Hoewel we andere masterstudies zijn gaan doen, hebben we wel altijd contact gehouden, middels wisselend frequente koffie- of lunchpauzes, of een barbecue met een biertje. Het viel me altijd op dat we goede gesprekken en discussies hadden, die soms als filosofisch beschouwd konden worden, maar waarbij er ook genoeg plek was voor geouwehoer. Uiteraard bedank ik je ook voor het zijn van mijn paranimf vandaag, waarbij je het nerdgehalte in de voorbereidingen (scripts schrijven voor het geautomatiseerd opstellen van e-mailuitnodigingen) voor een groot deel hebt bepaald.

Nanda, we begonnen als collega's en we zijn geworden tot wie wij nu zijn. Onze promoties kenden een faseverschuiving, maar er was genoeg overlap om elkaar in specifieke perioden, waarin we soms tegelijkertijd zaten, en bepaalde situaties, te steunen. Omdat ik met mijn project een aantal maanden achter je aan jitterde, was jij de eerste van ons twee die de administratieve molen in ging voor de uiteindelijke promotie. Omdat ik het al eens gezien had, kon ik een aanzienlijk deel van de regelactie in feedforward realiseren en hoefde ik maar weinig bij te sturen. Hiermee heb je me, waarschijnlijk vrij onbewust, goed geholpen. Wat ik nu leuk vind is dat je nerd genoeg bent om precies te begrijpen wat ik bedoel met het jargon in deze eerste zinnen en dat je waarschijnlijk nog wel even met mij in discussie gaat, omdat je vindt dat mijn metaforen eigenlijk niet helemaal kloppen. Ik heb tijdens het project dankbaar gebruikgemaakt van jouw nerd-zijn, omdat je mijn problemen snel kon begrijpen en mij verder kon helpen door de juiste vragen te stellen waar ik geen antwoord op had en die dus moest vinden. Ik vind het fijn dat we veelal dezelfde interesses hebben in de muziek, sport en met reizen en ik hoop dat nog heel lang met je te kunnen doen en delen. Ook hoop ik dat je het grote deel van mijn leven dat je nu bent, blijft. Bedankt voor het zijn van de leukste en liefste! 





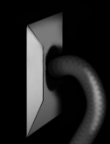

UNIVERSITY OF TWENTE. 


\title{
On the Modeling, Design, and Control of Compliant Robotic Manipulators
}

\author{
Propositions \\ Stefan Groothuis
}

1. The usage of variable stiffness actuators is the most versatile way to include compliance in robotic manipulators. - This thesis

2. The practical implementation and usage of a "single-motor" variable stiffness actuator is currently difficult due to the much more complicated mechanics. This thesis

3. Modeling compliant robotic manipulators using graph theory offers more flexibility than traditional modeling techniques. - This thesis

4. Being the first to research an interesting topic does not automatically necessitate nor approve carrying out that research.

5. The peak of acceptance of issues in a society is reached not when the achievements are frequently and explicitly raised in a sense of pride and contentment, but when those issues are not raised at all because they have been forgotten.

6. Our current consumer economy encouraging the acquisition of goods and services in ever-increasing amounts, and, thereby, pursuing an infinite economic growth, will fail on a planet with only a finite amount of resources.

7. The debate on how to cope with losing jobs as a consequence of a robotizing society, which in agreement with popular belief indeed occurs, should include the fundamental redesign of our society.

8. "Loosely speaking", "informally", "concretely", and "in other words" are music to the ears of a non-mathematician.

9. Although highly respectful, naming a mathematical topic after its inventor never contributes to easy understanding or memorizing of that topic.

10. Whatever the mind can conceive and believe, it can achieve. - Napoleon Hill 